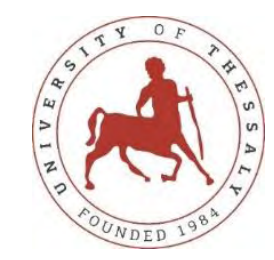

UNIVERSITY OF THESSALY

Faculty of Engineering

Department of Mechanical Engineering

\title{
Design and Modeling of Printed-Circuit Heat Exchangers and Centrifugal Compressors for Supercritical Carbon Dioxide
}

A thesis submitted for the degree of

Doctor of Engineering

Apostolos A. Gkountas

Dipl. Mechanical Engineer

Volos, April 2019

This research is co-financed by Greece and the European Union (European Social Fund- ESF) through the Operational Programme «Human Resources Development, Education and Lifelong Learning» in the context of the project "Strengthening Human Resources Research Potential via Doctorate Research" (MIS5000432), implemented by the State Scholarships Foundation (IKY).

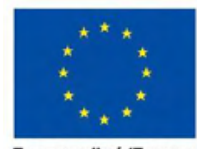

Eupwraïкń 'Evwon European Social Fund
Operational Programme Human Resources Development, Education and Lifelong Learning Co-financed by Greece and the European Union

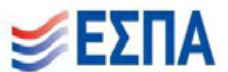
㫪国2014-2020

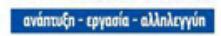




\section{Design and Modeling of Printed-Circuit Heat Exchangers and}

\section{Centrifugal Compressors for Supercritical Carbon Dioxide Power Cycles}

\section{Abstract}

The closed loop Brayton cycle using supercritical carbon dioxide $\left(\mathrm{s}-\mathrm{CO}_{2}\right)$ is a promising technology for waste heat recovery and power production. This thesis presents the design and performance modeling of the most critical cycle components, namely the printed-circuit heat exchangers and the centrifugal compressor.

The attainable limits of recompression Brayton cycle efficiency, using supercritical carbon dioxide, are investigated. A comprehensive study on the design and modeling of printed-circuit heat exchangers and the centrifugal compressor is used to achieve this goal. A case study of a thermodynamic analysis is used for a power cycle with a thermal load of $600 \mathrm{MW}$. The results indicate that thermal efficiency figures exceeding $46 \%$ are possible for a turbine inlet temperature of $830 \mathrm{~K}$. The supercritical carbon dioxide varies abruptly its properties, particularly close to the critical point. Therefore, a split cycle with recompression is used to reduce the entropy generation. The design of heat exchangers based on the printed-circuit technology are investigated. The components are modeled by using a one-dimensional segmental analysis, three-dimensional computational fluid dynamics analysis and experimental methods. The limits of attainable heat exchanger effectiveness and their effect on the total efficiency are investigated. The performed analysis validated by experimental data, suggests a high effectiveness design for the hightemperature recuperator.

The pressurized conditions of the supercritical carbon dioxide and the two-phase risk approaching the critical region, point the need to investigate the centrifugal compressor design and operation. Both direct and inverse problems are solved. The direct design problem is solved for a tested centrifugal compressor. The inverse problem is solved in order to obtain the compressor geometry that meets the necessary thermodynamic conditions. An iterative one-dimensional design model has been developed, aiming to estimate through the compressor efficiency. A computational fluid dynamics analysis is used, in order to investigate the flow field in the compressor, taking into account the real gas effects. The two-phase flow at the compressor inlet is examined, in view of identifying the local flow acceleration at the impeller leading edge. Moreover, the effect of tip clearance on the compressor performance and its impact on possible condensation is investigated. It has been shown that when tip clearance increases from $1 \%$ to $5 \%(1 \mathrm{~mm})$ of blade height, condensation occurs at the leading edge tip. The compressor performance map is derived and the condensation investigation leads to a limited operational range in order to ensure supercritical flow throughout the cycle.

The high-temperature recuperator with $99 \%$ effectiveness should lead to $0.1 \%$ cycle efficiency improvement. Moreover, the designed compressor's operational range is limited to a $30 \%$ of the initial range for low rotational speed and to a $25 \%$ for higher rotational speeds.

Thesis Co-supervisors:

Anastassios M. Stamatelos, Professor, University of Thessaly

Anestis I. Kalfas, Associate Professor, Aristotle University of Thessaloniki 


\section{Acknowledgements}

First of all, I would like to dedicate my thesis to my late Professor Tassos Stamatis, who initiated me to the field of Turbomachinery, for his contribution to my personality and the inspiration regarding the scientific research.

I would like to genuinely express my deep gratitude to my supervisors Professor Tassos Stamatelos and Associate Professor Anestis Kalfas for their valuable advice and guidance during these years. Professor Stamatelos stood by me when circumstances were complicated, offered me the opportunity to investigate the field I like and aided directly in my research providing the guidance that enabled me to successfully navigate difficult problems. My co-supervisor Anestis Kalfas was offered to help and advise me from my difficult starting point till the end. He has been the integral part of countless working discussions that helped me to expand my knowledge about turbomachinery.

Furthermore, I would like to thank Prof. Herrikos Stapountzis, an ex-member of my advisory committee for his useful suggestions, as well as the new member of the advisory committee Assistant Professor Georgios Charalampous for his beneficial comments on my research work.

I would like to express my acknowledgment to Dr. Pavlos Zachos for giving me the opportunity to work in a project of Rolls Royce UTC at Cranfield University, get involved with the experimental procedure and use data for my thesis. Moreover, I would like to thank Dr. Eduardo Anselmi for our cooperation and his enthusiastic support during the experiments at Cranfield University.

I would like to extend my thanks to Georgios Aretis, a MSc student at the University of Thessaly, for his valuable contribution on the compressor's CFD simulations, as well as Georgios Stamatellos, a graduate student at the Aristotle University of Thessaloniki, for the CFD simulations on printed-circuit heat exchangers. Of course, I would like to thank all my friends and colleagues at Aristotle University for their help and the pleasant time throughout my study. A special thanks to my friend Theofilos Efstathiadis for all the time we had together.

Last but not least, I would like to express my huge appreciation of my family, for the priceless support at each level and their emotional encouragement, and especially to my brother Nikos for the sharing of so many great times. The last words are for Efi, for her endless patience and support during all these years.

Finally, I would like to thank the Greek State Scholarships Foundation (IKY) for providing financial support for this $\mathrm{PhD}$ research.

Apostolos Gkountas 


\section{Contents}

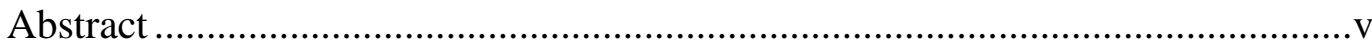

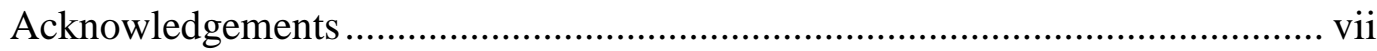

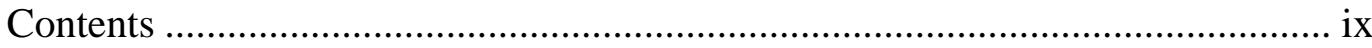

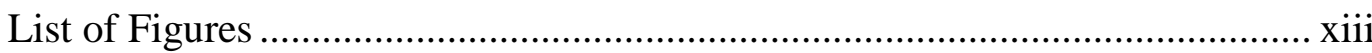

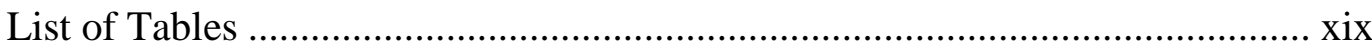

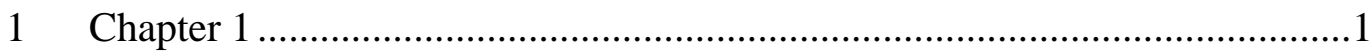

1.1 Supercritical $\mathrm{CO}_{2}$ Power Cycles.........................................................

1.2 Applications of s- $\mathrm{CO}_{2}$ Brayton Cycles.............................................

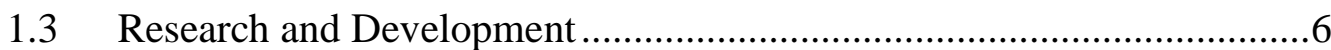

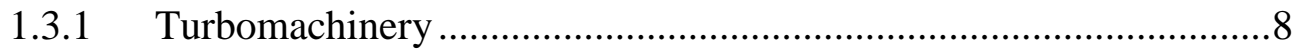

1.3.2 Commercial s- $\mathrm{CO}_{2}$ Power Cycles ................................................. 10

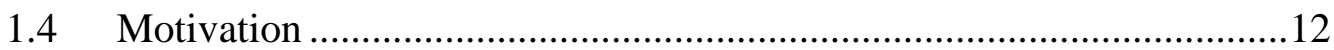

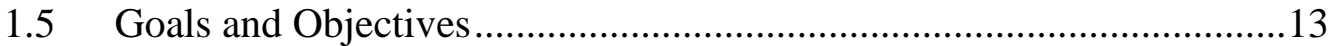

1.6 Contributions to Science and Technology..............................................15

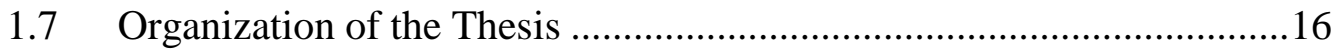

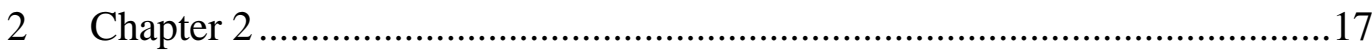

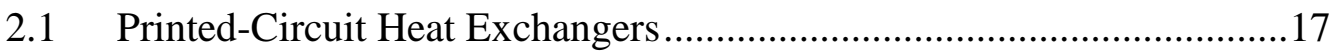

2.1.1 Steady state thermal-hydraulic performance of PCHE ..................21

2.1.2 Dynamic performance of PCHE ....................................................24

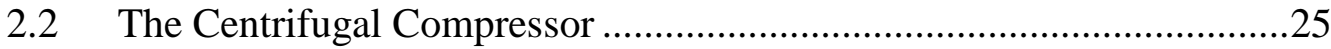

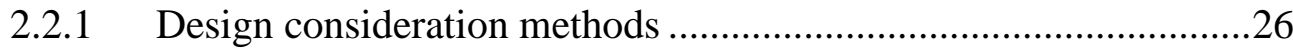

2.2.2 Condensation and two-phase flow investigation ............................30

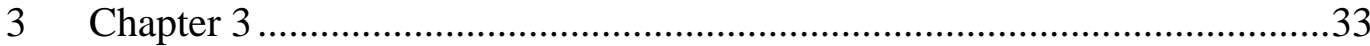

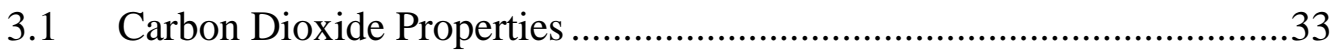

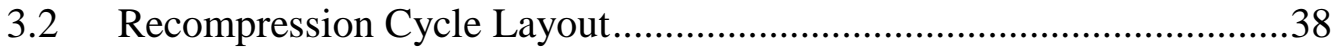

3.2.1 Compressor suction temperature and pressure ratio effect .............41

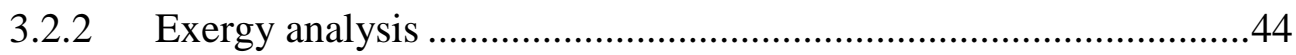

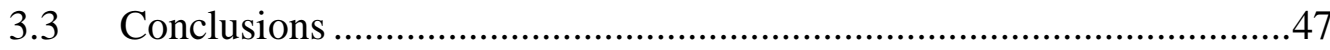




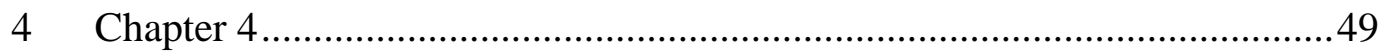

4.1 Selection of Heat Exchangers Design ..................................................49

4.2 Steady-State One-Dimensional Modeling............................................50

4.2.1 Overall heat transfer coefficient ....................................................

4.3 Dynamic Modeling for PCHE Performance .........................................55

4.4 Numerical Method ...................................................................................56

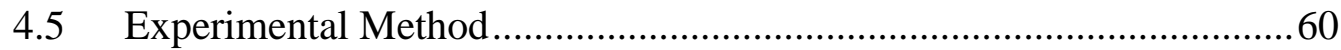

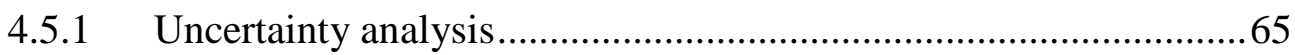

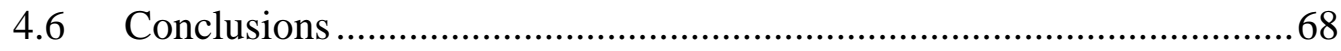

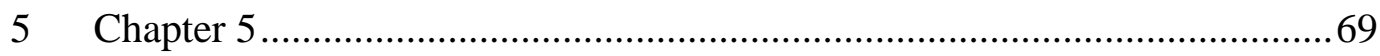

5.1 One-Dimensional Compressor Model................................................69

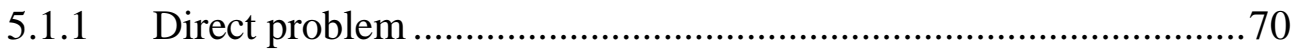

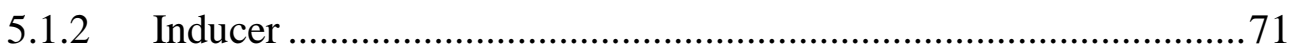

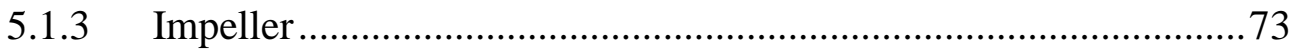

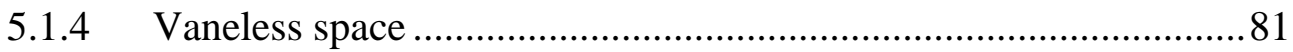

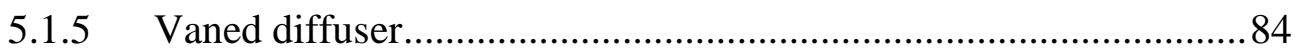

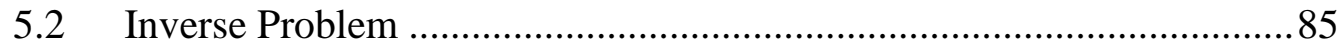

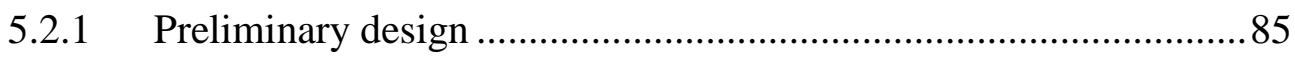

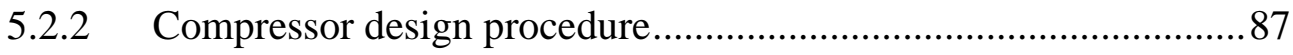

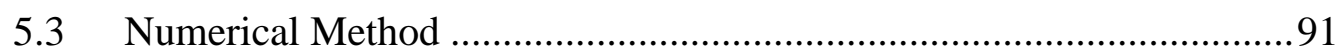

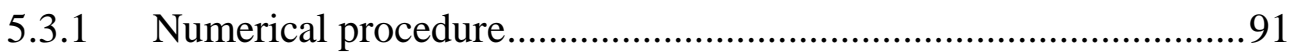

5.3.2 Geometry and Mesh generation .................................................92

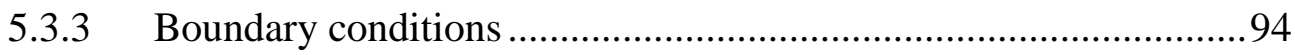

5.3.4 Real gas effects on numerical solution ........................................96

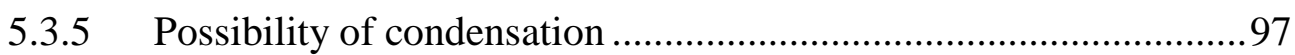

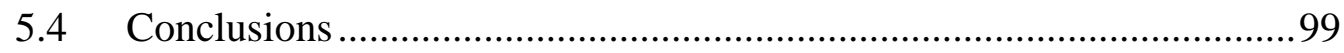

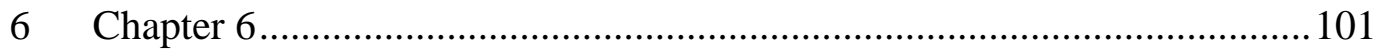

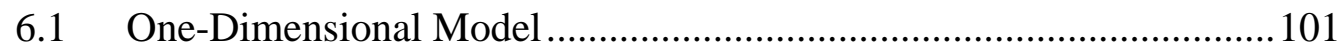

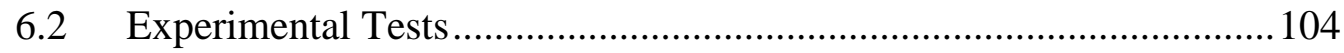

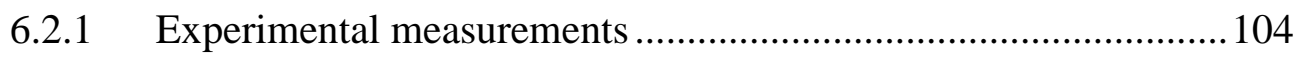




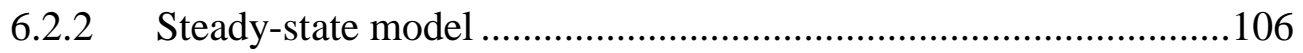

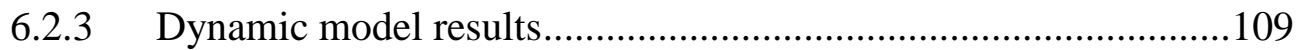

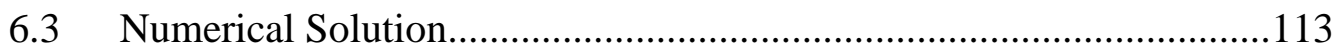

6.3.1 Numerical investigation of the tested cases .................................114

6.3.2 High-temperature recuperator investigation ................................122

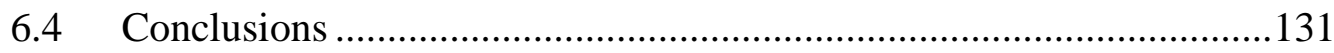

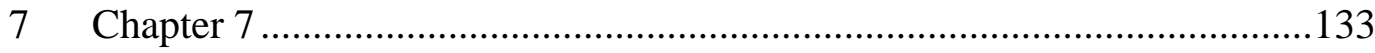

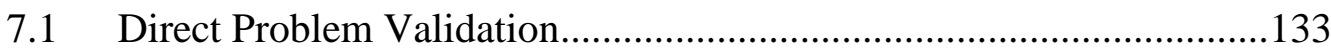

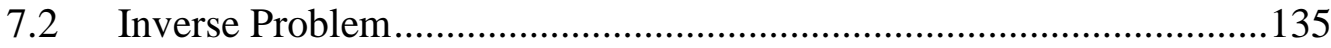

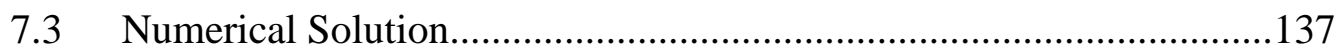

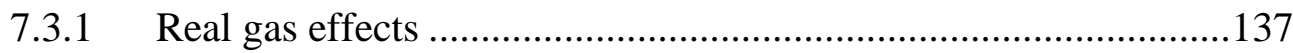

7.3.2 Aerodynamic analysis for design point........................................140

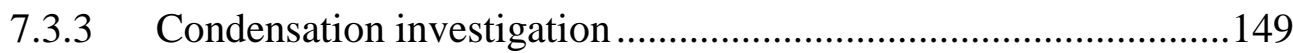

7.3.4 Tip clearance effect on condensation........................................... 153

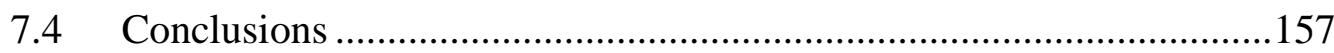

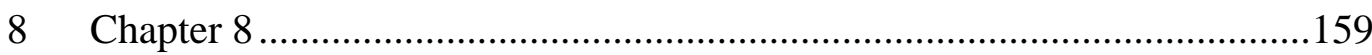

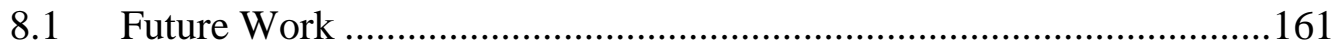

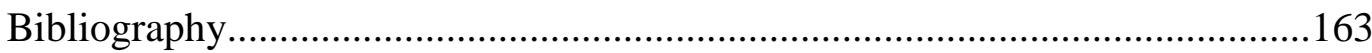

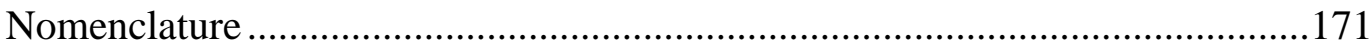

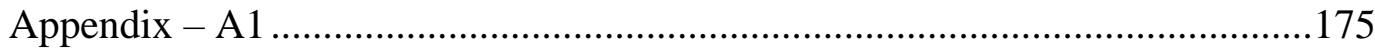

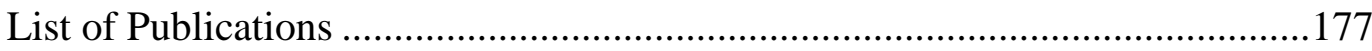




\section{List of Figures}

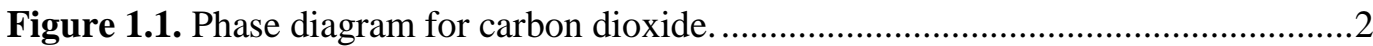

Figure 1.2. Various s- $\mathrm{CO}_{2}$ Brayton cycle layouts that were examined by Angelino [2]...2

Figure 1.3. Efficiency for competitive power cycles [4] ............................................... 3

Figure 1.4. Schematic diagram of a simplified direct $\mathrm{s}-\mathrm{CO}_{2}$ cycle [4] .......................... 4

Figure 1.5. Indirect configuration for solar-assisted s- $\mathrm{CO}_{2}$ Brayton power cycle.............6

Figure 1.6. Component and technology options for s- $\mathrm{CO}_{2}$ Brayton cycles for various power level systems [14] ....................................................................................

Figure 1.7. Centrifugal compressor's impeller developed by SNL [28] .........................9

Figure 1.8. Centrifugal compressor's impeller and radial turbine designed and operated

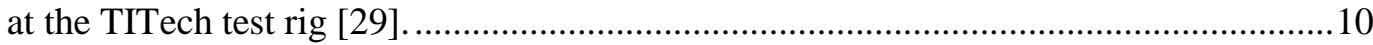

Figure 1.9. Process flow diagram of the EPS100 test facility [31] ..............................11

Figure 1.10. General arrangement of the coal Allam cycle for s- $\mathrm{CO}_{2}[30] . \ldots \ldots \ldots \ldots \ldots \ldots . . .12$

Figure 1.11. Thesis roadmap for the heat exchanger design and study.........................14

Figure 1.12. Roadmap for the compressor design and investigation..............................15

Figure 2.1. Structure of the PCHE's hot and cold plates [32] ....................................18

Figure 2.2. (a) Airfoil finned and (b) S-shaped finned channels for PCHE [33, 35].......18

Figure 2.3. Plate with straight and wavy channels and cross section of the PCHE [31,

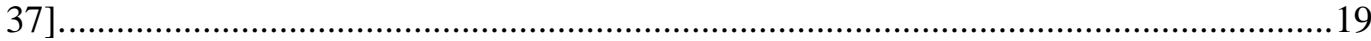

Figure 2.4. Parallel and offset configuration of plates in the PCHE [32].......................19

Figure 2.5. Pinch-point problem for heat exchanger. ....................................................... 21

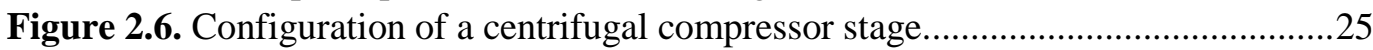

Figure 2.7. Velocity triangles at inlet and outlet of the impeller.....................................26

Figure 2.8. Balje diagram for compressors design [72]...........................................28

Figure 3.1. Specific heat-temperature and density-temperature diagrams of $\mathrm{CO}_{2}$ for various pressures.

Figure 3.2. Carbon dioxide speed of sound and compressibility factor variation with temperature for different pressures.

Figure 3.3. Specific heat capacity variation for Span-Wagner and Peng-Robinson

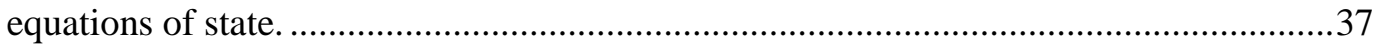

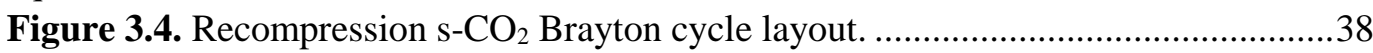

Figure 3.5. Temperature-entropy diagram for recompression s- $\mathrm{CO}_{2}$ Brayton cycle.......39 Figure 3.6. $\mathrm{Log} \mathrm{P}$-enthalpy diagram for recompression s- $\mathrm{CO}_{2}$ Brayton cycle, presented

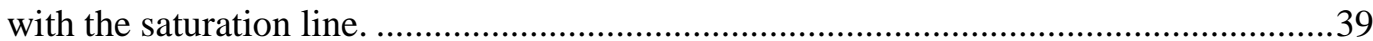

Figure 3.7. Thermal efficiency variation as compressor pressure ratio increases. ...........41

Figure 3.8. Recuperators' effectiveness as function of compressor inlet temperature. ...42 Figure 3.9. High \& low temperature recuperator effectiveness as function of compressor discharge pressure.

Figure 3.10. Efficiency improvement as turbine entry temperature increases for various cycle's maximum pressures. 
Figure 3.11. Temperature-exergy diagram of the cycle ...............................................

Figure 3.12. Exergy balance of the recompression s- $\mathrm{CO}_{2}$ Brayton cycle........................46

Figure 3.13. Thermal and exergy efficiency of the cycle as the turbine entry temperature

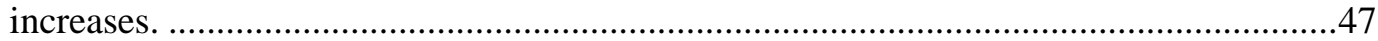

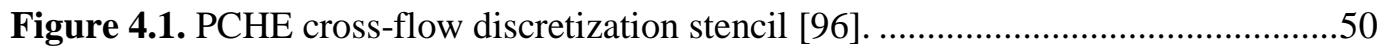

Figure 4.2. Geometrical characteristics of PCHE channels.........................................51

Figure 4.3. Detailed geometry of the designed PCHE channels....................................58

Figure 4.4. Geometry of the whole PCHE developed model. ........................................58

Figure 4.5. Mesh of printed-circuit heat exchanger channels.....................................59

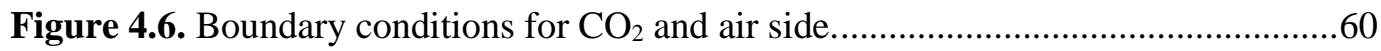

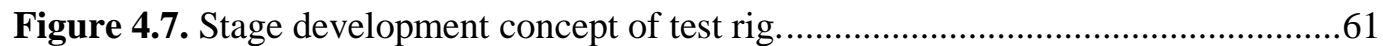

Figure 4.8. Trascritical compressors used for the test facility.....................................61

Figure 4.9. Piping and instrument diagram of the experimental setup.........................62

Figure 4.10. Components of experimental test rig for PCHE .........................................63

Figure 4.11. Difference between tubes employed for conventional and s- $\mathrm{CO}_{2}$ applications.

Figure 4.12. Schematic diagram of the (a) front and (b) side view of the tested PCHE..64

Figure 4.13. View of the (a) inlet and (b) outlet side of the tested PCHE.....................64

Figure 4.14. Normalized normal distribution of uncertainty for standard (66\% level of confidence) and extended uncertainty (95\% level of confidence) [104] .........................66

Figure 5.1. Enthalpy-entropy diagram for the centrifugal compressor stage. .................71

Figure 5.2. Impeller cross section, where incidence losses present...............................73

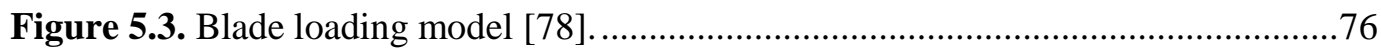

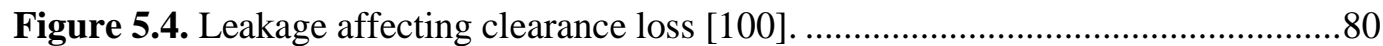

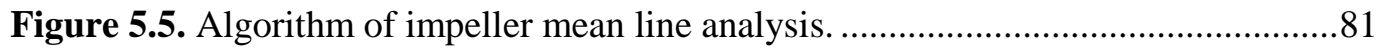

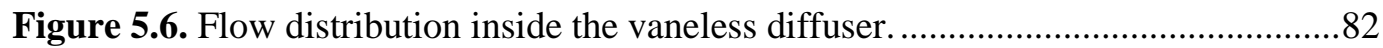

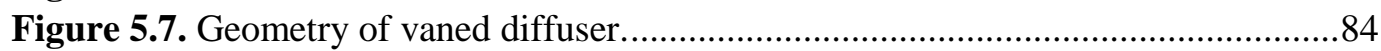

Figure 5.8. Centrifugal compressor design and analysis process. .................................85

Figure 5.9. Density change during compression procedure in temperature-density

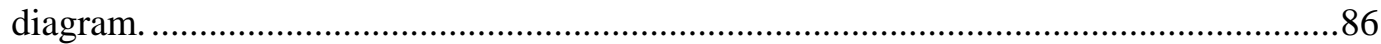

Figure 5.10. Typical values of flow and stage coefficients for radial and axial

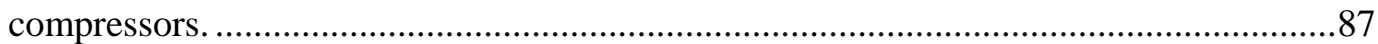

Figure 5.11. Estimation procedure for static variables for the compressor inducer. ........88

Figure 5.12. Flowchart for inverse problem solution. ……......................................... 90

Figure 5.13. Impeller geometry derived by inverse problem: a) full geometry, b) section

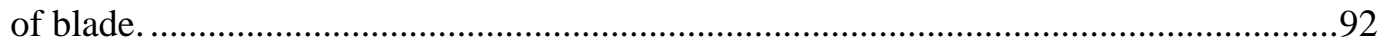

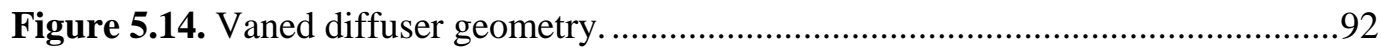

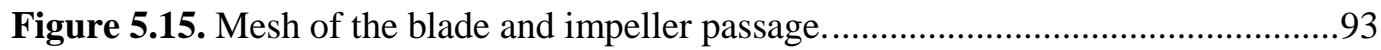

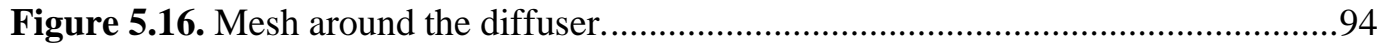

Figure 5.17. Location of the inlet and outlet periodic conditions and periodic interface.

Figure 5.18. Accuracy of specific heat in the pseudocritical region for pressure 7.69 MPa. 
Figure 5.19. Accuracy of specific heat in the pseudocritical region for pressure 10 and $15 \mathrm{MPa}$...... .97

Figure 5.20. Drop inside the two-phase region because of flow acceleration................99

Figure 6.1. Reynolds number as a function of heat exchanger length........................102

Figure 6.2. Nusselt number as a function of heat exchanger length............................ 103

Figure 6.3. NTU diagram for high \& low temperature recuperators............................103

Figure 6.4. Position of the four heat exchanger operating points on the general dimensionless diagram of counter-flow heat exchangers' performance [115].............105

Figure 6.5. Energy balance between hot $\left(\mathrm{s}-\mathrm{CO}_{2}\right)$ and cold (air) side of the heat exchanger............................................................................................. 106

Figure 6.6. Temperature distribution for the hot and cold side for Case 1 and 2........107

Figure 6.7. Nusselt number as function of Reynolds for various correlations. .............107

Figure 6.8. Pressure drop as percentage of inlet pressure measured cases and the corresponding predictions.

Figure 6.9. Heat transfer coefficient along heat exchangers for the four operating cases.

Figure 6.10. Mass flow variation with the time.

Figure 6.11. Dynamic behavior of $\mathrm{CO}_{2}$ outlet temperature for ramp variation of $\mathrm{CO}_{2}$ inlet temperature.

Figure 6.12. Dynamic behavior of air outlet temperature for experimental and numerical results during power-up.

Figure 6.13. Measured carbon dioxide mass flow rates step changes.

Figure 6.14. Dynamic behavior of $\mathrm{CO}_{2}$ inlet and outlet temperature for the step change.

Figure 6.15. Hot and cold stream temperatures for the heat exchanger and the module for the CFD computation for test case 3.

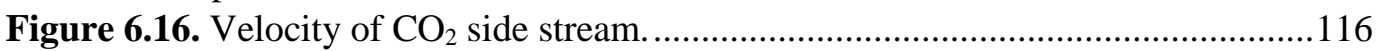

Figure 6.17. Velocity of air side stream. ........................................................... 116

Figure 6.18. Numerical solution for outlet temperature of air side. ...........................117

Figure 6.19. Numerical solution for outlet temperature of $\mathrm{CO}_{2}$ side.........................117

Figure 6.20. Temperature distribution in the $\mathrm{CO} 2$ and air passages along the channels.

Figure 6.21. Heat flux to air interface along the channel.

Figure 6.22. Heat exchanger outlet temperature of $\mathrm{s}-\mathrm{CO}_{2}$ for $\mathrm{CFD}$ solution and measured values.

Figure 6.23. Heat exchanger outlet temperature of air for CFD solution and measured values.

Figure 6.24. Numerically computed heat transfer coefficients for the four operation points and comparison with design point.

Figure 6.25. $\mathrm{S}-\mathrm{CO}_{2}$ inlet pressure derived by $\mathrm{CFD}$ and experiments. ........................ 121

Figure 6.26. Comparison of air inlet pressure at the heat exchanger module for CFD and experimental measurements.

Figure 6.27. Module for the CFD computation of the full scale HTR..........................123

Figure 6.28. Temperature distribution at low-pressure $\mathrm{CO}_{2}$ side outlet cross section...124 
Figure 6.29. Temperature distribution at high-pressure $\mathrm{CO}_{2}$ side outlet cross section.. 125 Figure 6.30. High-pressure $\mathrm{CO}_{2}$ side inlet pressure (reference pressure $20 \mathrm{MPa}$ ) ........125 Figure 6.31. Low-pressure $\mathrm{CO}_{2}$ side inlet pressure (reference value $7.7 \mathrm{MPa}$ )............ 126 Figure 6.32. $\mathrm{CO}_{2}$ outlet temperatures for high and low-pressure side of the recuperator as calculated by the CFD. 126

Figure 6.33. Average heat transfer coefficient for various mass flow rates per module.

Figure 6.34. NTU and effectiveness variation as function of mass flow per module estimated with CFD.

Figure 6.35. $\mathrm{CO}_{2}$ inlet pressure for high and low pressure side of designed recuperator as a function of mass flow per module and estimated with CFD.

Figure 6.36. Total heat exchange area and overall heat transfer coefficient of the specific HTR as function of mass flowrate per module, calculated by the CFD.

Figure 6.37. Nusselt number as function of Reynolds number for the designed HTR..130

Figure 7.1. Geometry of the tested Model A [116].

Figure 7.2. Share of the enthalpy losses of impeller.

Figure 7.3. Blade to blade view of Mach number for RGP table with (a) 500 points and

(b) 100 points resolution around the critical point.

Figure 7.4. Blade to blade view of density for RGP table with (a) 500 points and (b) 100 points resolution around the critical point.

Figure 7.5. View of one blade from inlet for condensation investigation with table resolution of (a) 500 and (b) 100 points.

Figure 7.6. Lateral view for total pressure contour for the whole compressor stage at span $50 \%$.

Figure 7.7. Lateral view for total temperature contour for the whole compressor stage at span $50 \%$.

Figure 7.8. Lateral view for velocity vectors for the compressor stage at span $90 \% \ldots 141$

Figure 7.9. Blade to blade view of (a) total pressure, (b) total temperature. 142

Figure 7.10. Blade to blade view of (a) relative Mach number, (b) absolute Mach number.

Figure 7.11. Blade to blade view of static pressure at mid-span.

Figure 7.12. Relative Mach number along the impeller passage .............................. 143

Figure 7.13. Relative velocity variation along the compressor. ............................... 144

Figure 7.14. Leakage flow at impeller tip............................................................ 145

Figure 7.15. Span-wise variation of static pressure close to throat. .............................145

Figure 7.16. Contours of vorticity along the designed impeller. ................................ 146

Figure 7.17. Contours of density at various planes along the impeller........................ 146

Figure 7.18. Compressor performance map for various rotational speeds. .................147

Figure 7.19. Efficiency-flow coefficient for varying rotational speed. ......................148

Figure 7.20. View of one blade from inlet indicating the temperature distribution at the impeller inlet $($ Tcrit, $\mathrm{CO} 2=304.1 \mathrm{~K})$.

Figure 7.21. View of one blade from inlet investigating condensation on the suction side of the impeller's blade for design point. 
Figure 7.22. View for one blade from inlet indicating the position of the pressure probes on the blade surface. 151

Figure 7.23. Pressure variation for probes at leading edge........................................152

Figure 7.24. Compressor performance map with condensation line-limit. 152

Figure 7.25. Blade to blade view of total pressure at span 0.9 for tip clearance (a) $1 \%$ and (b) $5 \%$ of blade height.

Figure 7.26. Blade to blade velocity for tip clearance (a) $1 \%$ and (b) $5 \%$ of the blade height. 154

Figure 7.27. Blade to blade entropy distribution for tip gap (a) $1 \%$ and (b) $5 \%$ of blade height. 155

Figure 7.28. Vorticity for various normalized chord lengths for tip gap (a) $1 \%$ and (b) $5 \%$ 155

Figure 7.29.View of one blade from inlet for condensation effect at blade for tip clearance (a) $1 \%$ and (b) $5 \%$ of the blade height. 


\section{List of Tables}

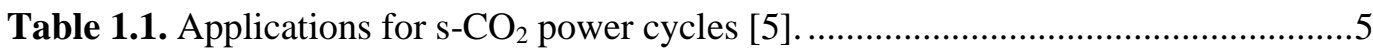

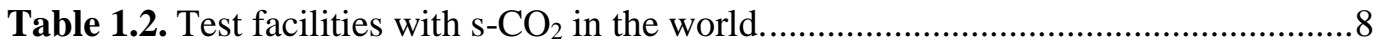

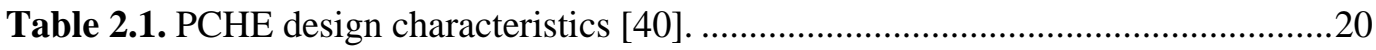

Table 2.2. Loss models adopted for centrifugal compressor design...............................29

Table 3.1. Deviation of density between Span-Wagner model and other equations of

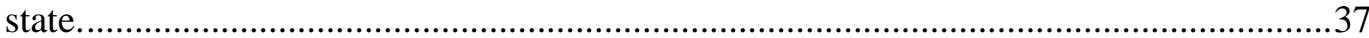

Table 3.2. Input design parameters for the recompression cycle....................................40

Table 3.3. Results for each operating point of the recompression cycle..........................40

Table 3.4. Operating characteristics of the power system for the design parameters ......44

Table 3.5. Exergy losses for each component of the recompression $\mathrm{s}-\mathrm{CO}_{2}$ Brayton cycle.

Table 4.1. Design parameters for the $\mathrm{s}-\mathrm{CO}_{2}$ rig test facility.....................................62

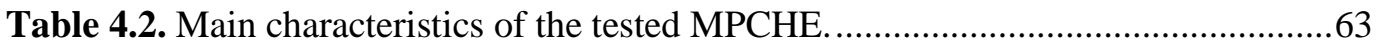

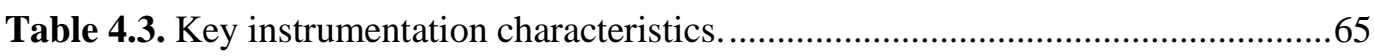

Table 5.1. Outlet thermodynamic properties variation with the mesh refinement...........94

Table 6.1. Specifications of Printed-Circuit Heat Exchangers. .......................................101

Table 6.2. Test cases of the printed-circuit heat exchanger operation............................104

Table 6.3. Test cases validated by means of CFD. ...................................................114

Table 6.4. Heat flux and heat transfer coefficient estimated by means of CFD for the test

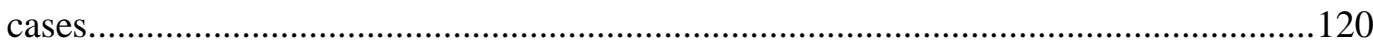

Table 6.5. High-temperature recuperator performance derived by means of CFD........124

Table 6.6. Geometrical characteristics of the designed high-temperature recuperator..130

Table 7.1. Centrifugal compressor geometry Model A, used as input for direct problem.

Table 7.2. Current model results validation with Model A operating conditions..........134

Table 7.3. Results for thermodynamic conditions at inlet and outlet of impeller..........135

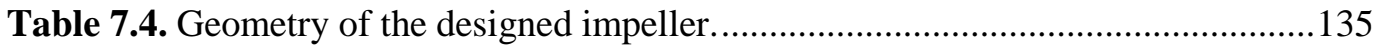

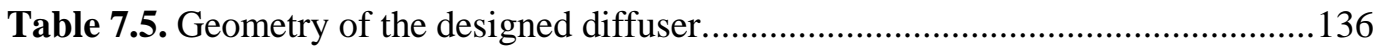

Table 7.6. Design characteristics comparison of centrifugal compressors for s- $\mathrm{CO}_{2}$ cycle. 136

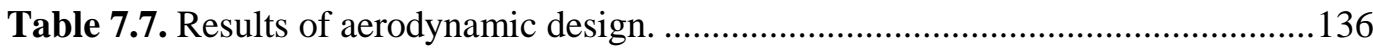

Table 7.8. Validation between 1D meanline and CFD results.....................................140 


\section{Chapter 1}

\section{Introduction}

Despite efforts to transform the world from coal-burning energy to clean energy during the last decades, fossil fuels remain the primary source of energy worldwide still producing almost $40 \%$ of carbon dioxide $\left(\mathrm{CO}_{2}\right)$ emissions. Therefore, a key challenge in power production is reducing the $\mathrm{CO}_{2}$ emissions and therefore the negative impact on environment. One way to accomplish this challenge is to capture carbon dioxide existing in the atmosphere and compress it under very high pressures reaching supercritical state. At this state, $\mathrm{CO}_{2}$ can be a suitable working medium for a range of applications, because its properties vary abruptly near the critical point $(304.13 \mathrm{~K}, 7.39 \mathrm{MPa})$. The low critical point of $\mathrm{CO}_{2}$ makes it suitable for a range of alternative heat sources regarding the power conversion systems, such as nuclear, solar and geothermal energy. Moreover, carbon dioxide presents plenty of advantages as a working fluid, because of its environmental friendliness, non-toxicity and compatibility with the majority of materials. Another way to reduce carbon dioxide emissions is to establish more efficient power systems that can reduce the fuel consumption.

\subsection{Supercritical $\mathrm{CO}_{2}$ Power Cycles}

The two most well-known cycles for power conversion are the Rankine cycle and the Brayton cycle with two different layouts, the open and the closed loop. Various working mediums have been used for both types of power conversion cycles. During the last decade, carbon dioxide concentrates a lot of research interest regarding the more mature technology of steam turbines (Rankine) or other more common working fluid for closed Brayton cycles, such as helium. The fact that closed Brayton cycle operating with supercritical carbon dioxide combine a high efficiency over a wide range of operating conditions and heat sources and high compactness, make it a very promising power conversion technology (Figure 1.1).

Carbon dioxide power cycles were firstly studied by Feher [1] in 1968 by investigating working medium cycles working above the critical point and a year later Angelino [2] examined various layouts providing performance results for each configuration and concluding that the recompression cycle can achieve comparable efficiency with that of a Rankine power cycle (Figure 1.2). 


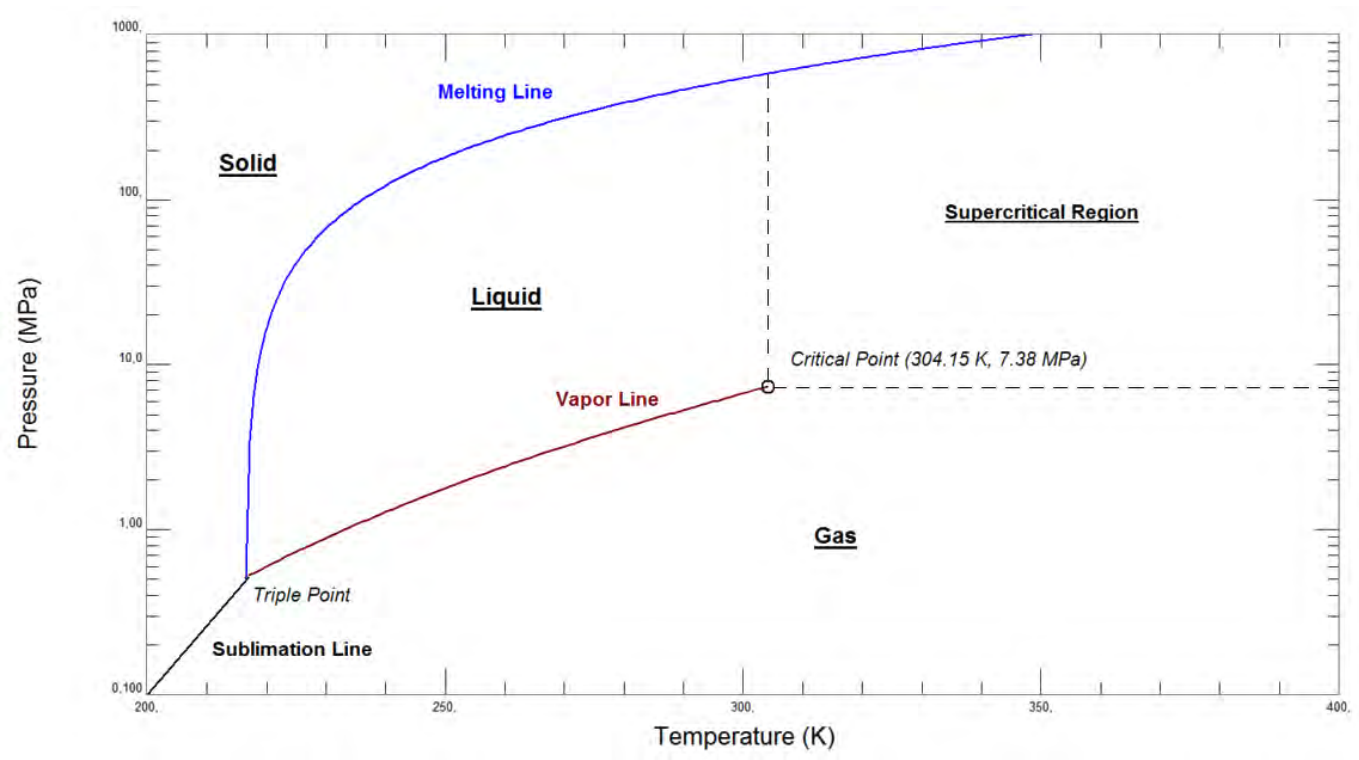

Figure 1.1. Phase diagram for carbon dioxide.

Angelino calculated that for a cooling water temperature of $5^{\circ} \mathrm{C}$ and turbine inlet temperature of $700^{\circ} \mathrm{C}$, a cycle efficiency in excess of $50 \%$ was achievable. In the next decades, the research regarding $\mathrm{s}-\mathrm{CO}_{2}$ as a possible working medium for power cycles was frozen, because of the lack of high temperature heat sources and the equipment that could guarantee a safe operation in high pressure and temperature.
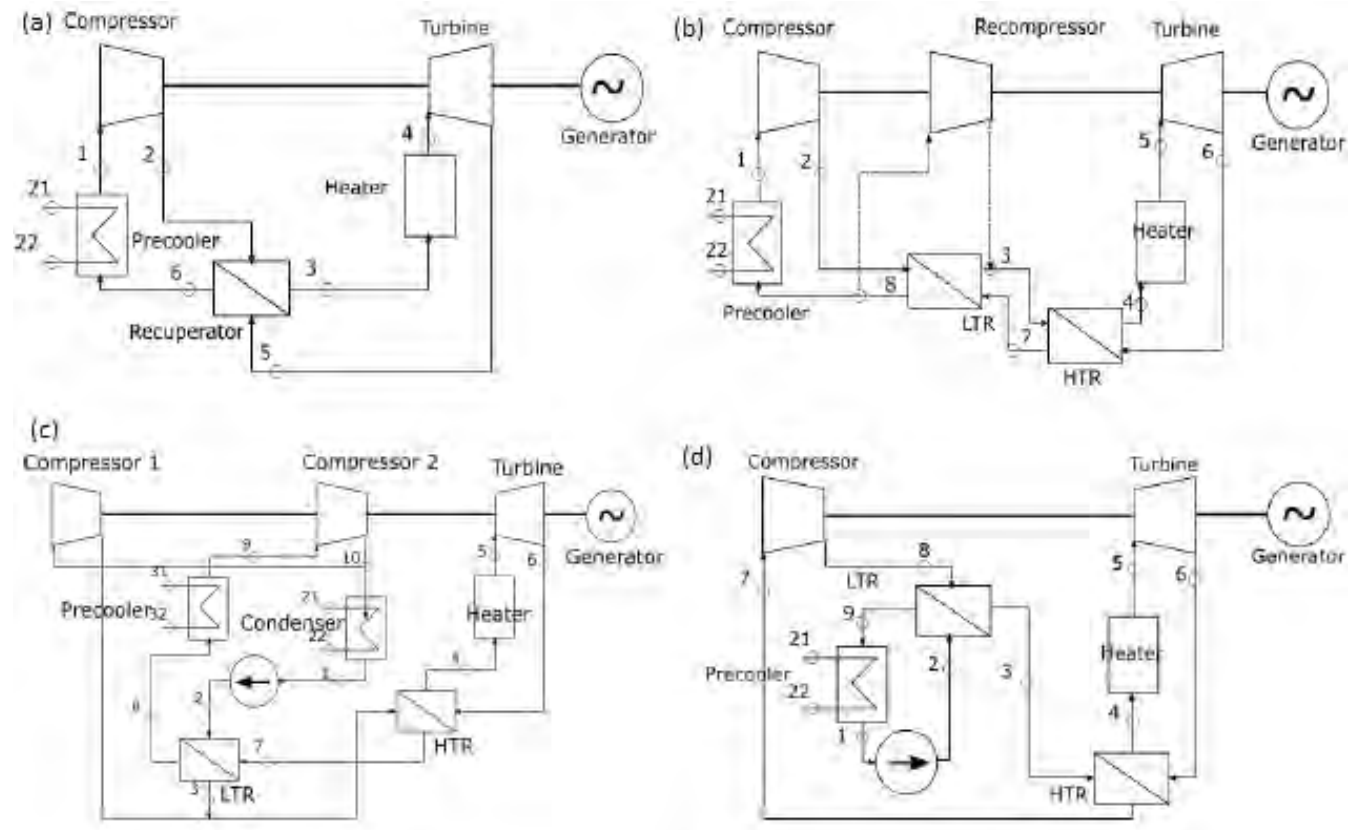

Figure 1.2. Various s- $\mathrm{CO}_{2}$ Brayton cycle layouts that were examined by Angelino [2]. 
However, since 90's carbon dioxide is already known in the oil and gas and refrigeration industry application, while due to the F-gas regulation (2007) the refrigeration industry has been investigating technologies using R744 refrigerant $\left(\mathrm{CO}_{2}\right)$ and appropriate equipment has been designed that can operate carbon dioxide in transcritical or supercritical state.

Therefore, using the experience form refrigeration and food industry, carbon dioxide cycles are getting attention also in the power generation technologies. The revival of interest for supercritical $\mathrm{CO}_{2}$ Brayton cycles performed at the beginnings of 2000's, when Dostal [3] revitalized the coupling of a s- $\mathrm{CO}_{2}$ Brayton cycle with a nuclear reactor. Since then, much of research work concentrates on s- $\mathrm{CO}_{2}$ Brayton cycles modeling, components designing and coupling with alternative heat sources.

In comparison with the conventional Rankine cycles that use steam or the helium or argon Brayton cycles that were studied in the past for nuclear applications, supercritical carbon dioxide Brayton cycles present higher efficiency in a wide temperature range, while demands lower heat addition, so it can cover more waste heat recovery applications . Additionally, carbon dioxide is a fluid in abundance and $\mathrm{CO}_{2}$ capture and storage technologies are more cost effective comparatively to helium and argon. Moreover, the supercritical $\mathrm{CO}_{2}$ cycle does not present less leakage than noble gases Brayton cycles, due to the three-atomic structure $\mathrm{CO}_{2}$ and its higher molecular weight.

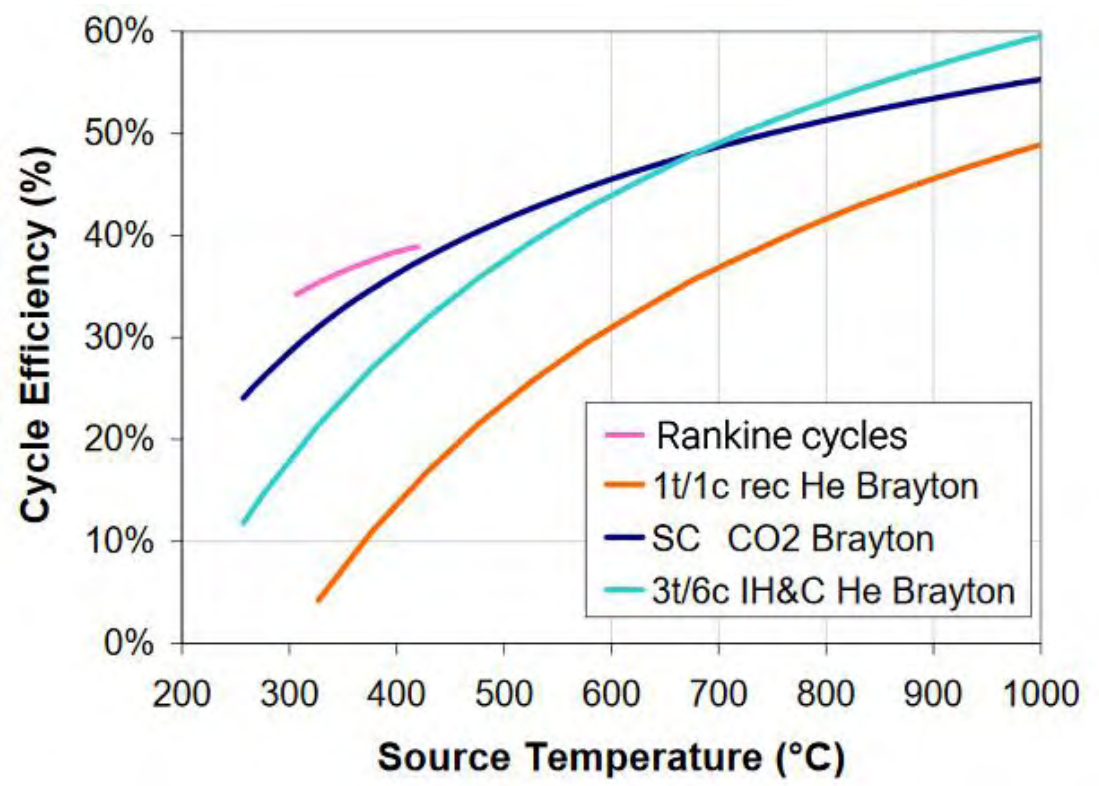

Figure 1.3. Efficiency for competitive power cycles [4]. 
Both helium and s- $\mathrm{CO}_{2}$ cycles require low pressure ratio compression (2-2.5). Although the considered mass flow rate for helium cycles is about 10 times less for given power generation, the density difference for the corresponding operating conditions explains that the facility variations are not significant between the two technologies. The density at the turbine exit for s- $\mathrm{CO}_{2}$ can be about 10000 times greater than for a condensing steam turbine and over 100 times that of combustion gas turbines that operate on an open Brayton cycle. This compactness reduces material costs and is also beneficial in low-weight or low-space applications, such as marine or solar (up-tower) power generation.

\subsection{Applications of s- $\mathrm{CO}_{2}$ Brayton Cycles}

Supercritical carbon dioxide cycle present a high efficiency over a range of temperatures that make it appropriate for numerous heat sources and industrial applications. These heat sources can be divided in two main categories: indirectly heated and directly-fired cycles. A closed-loop, indirectly heated $\mathrm{S}-\mathrm{CO}_{2}$ Brayton cycle (such as the cycles presented in Figure 1.2) is applicable to most thermal energy sources, such as fossil fuel combustion, nuclear, solar, geothermal and waste heat recovery. The directly-fired s- $\mathrm{CO}_{2}$ Brayton cycle (oxy-fuel cycle) comprises another approach for power generation with the use of supercritical carbon dioxide. In this approach, the $\mathrm{CO}_{2}$ heater is replaced with a pressurized oxyfuel combustor (Figure 1.4). Fuel is burned in relatively pure and near stoichiometric oxygen in the combustor, and the resulting stream, which contains mainly $\mathrm{CO}_{2}$ and $\mathrm{H}_{2} \mathrm{O}$, is used to drive the turbine.

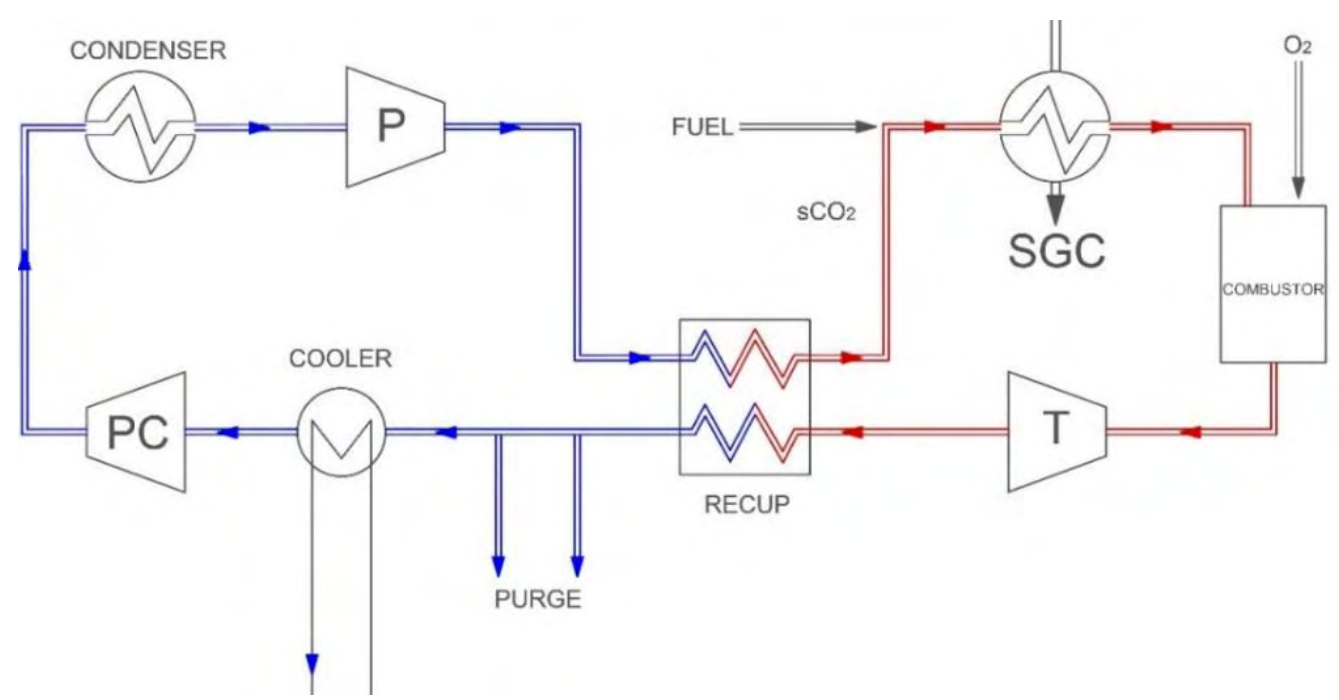

Figure 1.4. Schematic diagram of a simplified direct s- $\mathrm{CO}_{2}$ cycle [4]. 
The remaining heat in the stream exiting the turbine is recuperated and the stream is then further cooled to condense the water out, leaving a stream of high concentration $\mathrm{CO}_{2}$. A portion of the $\mathrm{CO}_{2}$ is compressed under the desired pressure. The cooled and compressed $\mathrm{CO}_{2}$ passes through the recuperator that it is preheated and it is then recycled to the combustor as diluent. The remainder of the $\mathrm{CO}_{2}$ is ready to be compressed for storage.

The most significant benefit of the $\mathrm{s}-\mathrm{CO}_{2}$ power cycle is the high thermal efficiency at moderate temperatures due to the low compression work and high amount of heat in the turbine exhaust that is recuperated and converted into work. The high-power density of s- $\mathrm{CO}_{2}$ enables size reduction of the systems, leading to a reduced plant footprint and lower capital costs. Furthermore, the low-pressure ratio of the turbine leads to a reduced number of required stages. For this reason, $\mathrm{s}-\mathrm{CO}_{2}$ Brayton cycle can be combined with various heat sources and applications.

Nuclear energy as a heat source was the first application that was examined for supercritical carbon dioxide, for both direct and indirect cycles. Since then, many countries have developed research interest on s- $\mathrm{CO}_{2}$ Brayton cycles, such as USA, Japan, China and South Korea. The main reason for these studies was the higher temperatures that fourth generation reactors can offer, which cannot be adopted by the power conversion system with a Rankine cycle. Closed Brayton cycles are a promising technology for these applications. Table 1.1 concentrates all the applications that could take place in $\mathrm{s}-\mathrm{CO}_{2}$ Brayton cycles and the respective range of operating conditions and power output.

Table 1.1. Applications for s- $\mathrm{CO}_{2}$ power cycles [5].

\begin{tabular}{|c|c|c|c|c|c|}
\hline Application & Cycle type & Benefits & $\begin{array}{c}\text { Size } \\
\text { (MWe) }\end{array}$ & $\begin{array}{c}\text { Temperature } \\
\left({ }^{\circ} \mathrm{C}\right)\end{array}$ & $\begin{array}{l}\text { Pressure } \\
\text { (MPa) }\end{array}$ \\
\hline Nuclear & Indirect $\mathrm{s}-\mathrm{CO}_{2}$ & $\begin{array}{l}\text { Efficiency, size, } \\
\text { water reduction }\end{array}$ & $10-300$ & $350-700$ & $20-35$ \\
\hline $\begin{array}{l}\text { Fossil fuel (such } \\
\text { as PC, CFB) }\end{array}$ & Indirect $\mathrm{s}-\mathrm{CO}_{2}$ & $\begin{array}{l}\text { Efficiency, water } \\
\text { reduction }\end{array}$ & $300-600$ & $550-750$ & $15-35$ \\
\hline $\begin{array}{l}\text { Concentrating } \\
\text { solar power }\end{array}$ & Indirect $\mathrm{s}-\mathrm{CO}_{2}$ & $\begin{array}{l}\text { Efficiency, size, } \\
\text { water reduction }\end{array}$ & $10-100$ & $500-700$ & 35 \\
\hline $\begin{array}{l}\text { Shipboard } \\
\text { propulsion }\end{array}$ & Indirect $\mathrm{s}-\mathrm{CO}_{2}$ & Efficiency, size & $10-100$ & $200-300$ & $15-25$ \\
\hline $\begin{array}{l}\text { Waste heat } \\
\text { recovery }\end{array}$ & Indirect $\mathrm{s}-\mathrm{CO}_{2}$ & $\begin{array}{l}\text { Efficiency, size, } \\
\text { simple cycles }\end{array}$ & $1-10$ & $230-650$ & $15-35$ \\
\hline Geothermal & Indirect s- $\mathrm{CO}_{2}$ & Efficiency & $1-50$ & $100-300$ & 15 \\
\hline $\begin{array}{l}\text { Fossil fuel } \\
\text { (syngas, natural } \\
\text { gas) }\end{array}$ & Direct s- $\mathrm{CO}_{2}$ & $\begin{array}{l}\text { Efficiency, water } \\
\text { reduction, } \mathrm{CO}_{2} \\
\text { capture }\end{array}$ & $300-600$ & $1100-1300$ & 35 \\
\hline
\end{tabular}




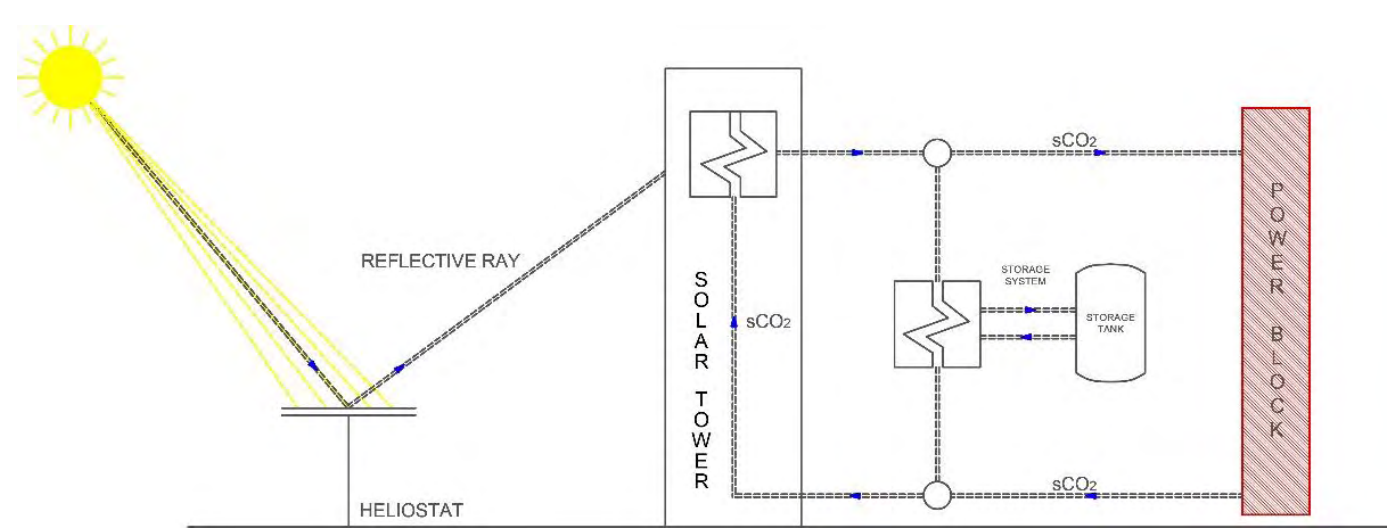

Figure 1.5. Indirect configuration for solar-assisted s- $\mathrm{CO}_{2}$ Brayton power cycle.

Solar energy as an alternative heat source has been investigated as a possible technology that could utilize the s- $\mathrm{CO}_{2}$ Brayton cycle for power generation (Figure 1.5), with many research studies on this field [6-12]. In this application, the great advantage of this kind of cycles is the low requirement for cooling water comparatively to steam cycles. For direct cycle solar applications, the usual design temperature of turbine inlet is $750^{\circ} \mathrm{C}$ set by the materials' properties limitations. For indirect solar plants the maximum temperature of the cycle depends on molten salts' properties, with a maximum possible temperature of $580^{\circ} \mathrm{C}$. Marine applications consist also another heat source that could employ a supercritical carbon dioxide cycle, reducing the fuel consumption.

Moreover, geothermal energy, fuel cells and waste heat applications can be combined with supercritical $\mathrm{CO}_{2}$ Brayton cycle. Finally, a supercritical carbon dioxide Brayton closed-loop has been lately studied by Rolls Royce as a bottoming power cycle for aero engine applications, using high enthalpy gases and resulting in lower specific fuel consumption of the gas turbine [13].

\subsection{Research and Development}

Supercritical $\mathrm{CO}_{2}$ cycles take advantage of the $\mathrm{CO}_{2}$ properties variation near the critical point. However, the same variation in properties present challenges for accurate modeling, design, and performance predictions of s- $\mathrm{CO}_{2}$ cycles and their individual components. Moreover, the commercialization of supercritical $\mathrm{CO}_{2}$ power cycle requires the development of reliable and affordable components, such as generator, instrumentation and heat exchangers, but also design and control methods that can assure the efficient operation of the power plant with minimum risk. 


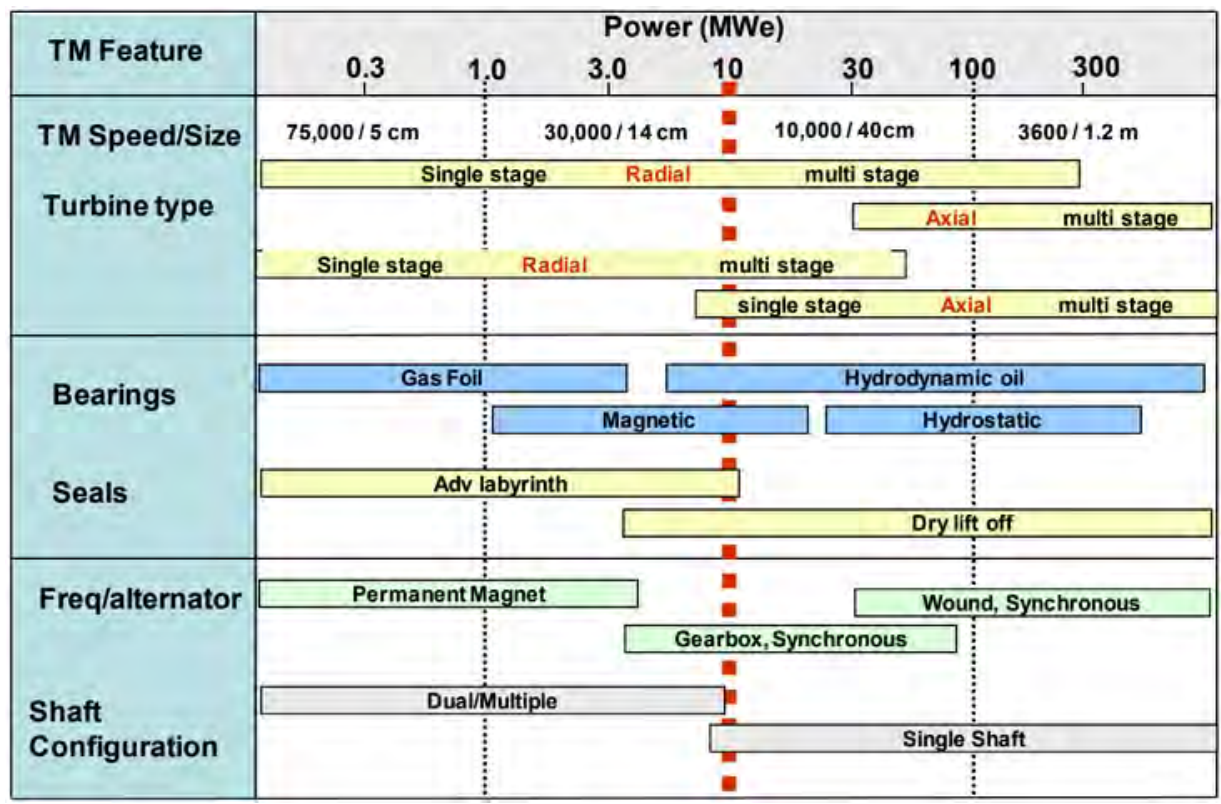

Figure 1.6. Component and technology options for s- $\mathrm{CO}_{2}$ Brayton cycles for various power level systems [14].

The majority of the equipment that needs to be employed in $\mathrm{s}-\mathrm{CO}_{2}$ power cycle is already in use in many industrial applications, although there are many components or control methods that cannot be considered mature and demand to be further researched. Depending on the size of the s- $\mathrm{CO}_{2}$ power plant, the options and the technology of the components may vary. Figure 1.6 summarizes some alternative options for the basic components categorized by the power required.

Since 2012, many research groups have been working on supercritical $\mathrm{CO}_{2}$ Brayton power cycles. For example, SANDIA National Laboratories have been working on a project for power production of $10 \mathrm{MWe}$, while the Bettis Atomic Power Laboratory in USA has built a $100 \mathrm{kWe}$ test facility to investigate the cycle operation over a range of conditions, for steady-state and dynamic performance $[15,16]$. In 2014, the Department of Energy of USA funded various projects in order to develop further the s- $\mathrm{CO}_{2}$ power cycles. In 2016, the US DOE invested a further 30 million dollars in projects developing components for advanced turbine and supercritical $\mathrm{CO}_{2}$ power cycles. The majority of the projects included GE Global Research (USA) and SWRI. Later, in October 2016, the US DOE announced the investment of 80 million dollars for a six-year project to design and operate a $10 \mathrm{MWe} \mathrm{S}-\mathrm{CO}_{2}$ pilot plant facility, which was carried out by a team including Gas Technology Institute (GTI), SWRI and GE Global Research.

The $\mathrm{s}-\mathrm{CO}_{2}$ cycles have been also studied in Japan (Tokyo Institute of Technology), where researchers have been studying the incorporation of these cycles for nuclear plants. Various $\mathrm{s}-\mathrm{CO}_{2}$ cycle configurations were studied including designs of s- $\mathrm{CO}_{2}$ turbomachinery and recuperators. 
Table 1.2. Test facilities with $\mathrm{s}-\mathrm{CO}_{2}$ in the world.

\begin{tabular}{ccccl}
\hline Year & Research group & Country & Power load & Application \\
\hline 2010 & Sandia NL & USA & $260 \mathrm{MW}_{\text {th }}$ & Nuclear \\
2010 & Sandia NL & USA & $1 \mathrm{MW}_{\text {th }}$ & Nuclear \\
2010 & Echogen ES & USA & $6-8 \mathrm{MW}_{\mathrm{e}}$ & WHR \\
2010 & TITech & Japan & $10 \mathrm{~kW}_{\mathrm{e}}$ & General \\
2013 & KAERI-KAIST & South Korea & $80 \mathrm{~kW}_{\mathrm{e}}$ & Nuclear \\
2013 & KIER & South Korea & $730 \mathrm{~kW}_{\text {th }}$ & Nuclear \\
2015 & Czech TU & Czech Republic & $500 \mathrm{~kW}_{\text {th }}$ & General \\
\hline
\end{tabular}

In June 2007, a three-year project funded by the Japanese government began at the Tokyo Institute of Technology, with the construction of a small centrifugal compressor test loop and cycle performance studies. Moreover, their research was expanded at applications that include fossil fuel plants and the study of axial compressors [17-20].

Furthermore, significant research regarding $\mathrm{s}-\mathrm{CO}_{2}$ technology takes place in South Korea, mainly at the Korea Atomic Energy Research Institute (KAERI) and Korea Advanced Institute of Science \& Technology (KAIST). In 2013, KAERI designed and constructed a $300 \mathrm{kWe}, \mathrm{S}-\mathrm{CO}_{2}$ compressor test loop, the s- $\mathrm{CO}_{2}$ Integral Experiment Loop (SCIEL), to investigate technologies regarding $\mathrm{s}-\mathrm{CO}_{2}$ power generation systems. The first tests focused on a centrifugal compressor performance and the cycle control, while in 2015 the test facility was upgraded to a closed test loop for power generation [21-24].

Also, some projects have been lately funded in Europe, such as I-ThERM $\mathrm{CO}_{2}$ project that investigates waste heat recovery solutions in the temperature range of $70^{\circ}-1000^{\circ}$, and $\mathrm{sCO} 2-$ Hero and following the sCO2-Flex investigating the heat removal from a nuclear reactor and developing two test loops, one in Stuttgart (Germany) and another in Plzen (Czech). Moreover, in 2014 the Seville University in Spain was funded in order to study a $10 \mathrm{MW}_{\mathrm{e}} \mathrm{CSP}$ plant. Finally, in 2018, the first test rig in United Kingdom was developed and run at Cranfield University in a project that co-funded by Rolls-Royce plc, investigating the possibility of s- $\mathrm{CO}_{2}$ cycle in marine applications.

\subsubsection{Turbomachinery}

The turbomachinery in these cycles is quite different from that met in conventional Brayton cycles or Rankine cycles. Compared to perfect gas Brayton cycles these turbomachines have a large mass flow rate, a considerable bending stress, a low Mach number and are very compact. Due to the highly pressurized conditions of $\mathrm{CO}_{2}$, the specific enthalpy change is much smaller for given a 
pressure ratio, which translates into reduced compression work and the potential for higher efficiency.

Over the past two decades, laboratory-scale s- $\mathrm{CO}_{2}$ cycle test loops have been assembled, and designs and fabrication methods developed and validated, for S$\mathrm{CO}_{2}$ turbomachinery including bearings, seals and alternators [14, 25, 26]. A single stage radial turbine was used in all these small-scale test facilities.

The high-energy density of the $\mathrm{S}-\mathrm{CO}_{2}$ power cycle shows that at $125 \mathrm{kWe}$, each turbine and compressor wheel will have a very small size (only a few centimeters diameter) leading in high shaft speeds (around $75000 \mathrm{rpm}$ ) that require specialized approaches for bearings, seals, and alternators. The turbines and compressors operating at test facilities have performed close to the design maps and have operated smoothly both below and above the critical temperature [27].

Since May 2008, the Sandia National Laboratories (SNL) have developed a small scale compression loop for supercritical carbon dioxide, in order to evaluate the performance of such turbomachinery, mainly for nuclear applications [28]. The facility was designed and operated by SNL and Barber-Nichols Inc. using a 50 kWe motor-driven compressor. Later, in January 2009 a radial turbine of 1.2-inch diameter was added to the test rig, in order to investigate the turbine temperature inlet.

Moreover, a research group in Tokyo Institute of Technology (TITech) developed a small scale supercritical $\mathrm{CO}_{2}$ test rig, operating with a turbine inlet temperature up to $550 \mathrm{~K}$ (Figure 1.8). The main results of the study focus on the aerodynamic effect of compressor on the performance of a $18 \mathrm{~kW}$ cycle and the verification of the meanline tool that was developed by the group [29].

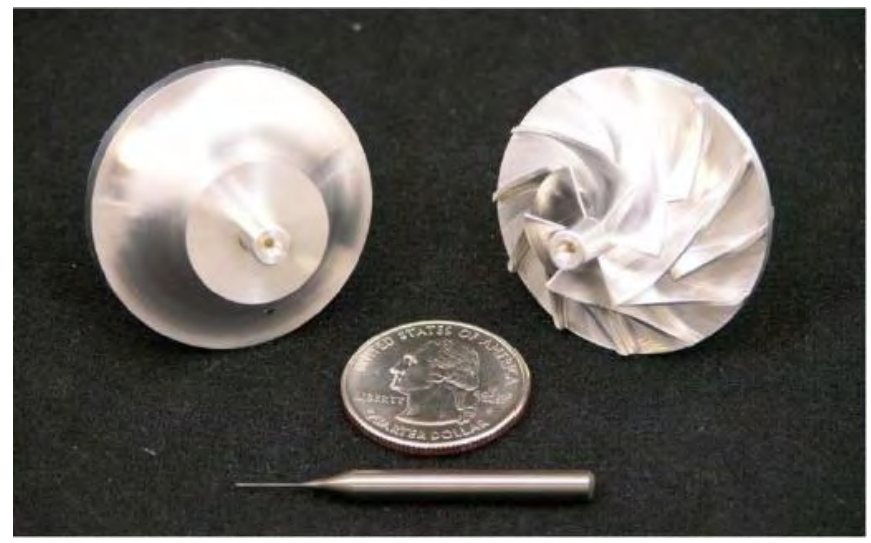

Figure 1.7. Centrifugal compressor's impeller developed by SNL [28]. 


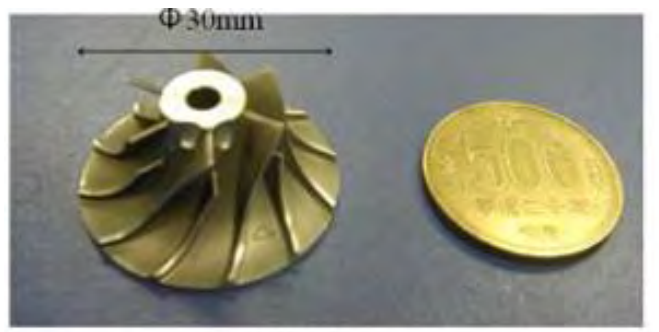

Centrifigal compressor

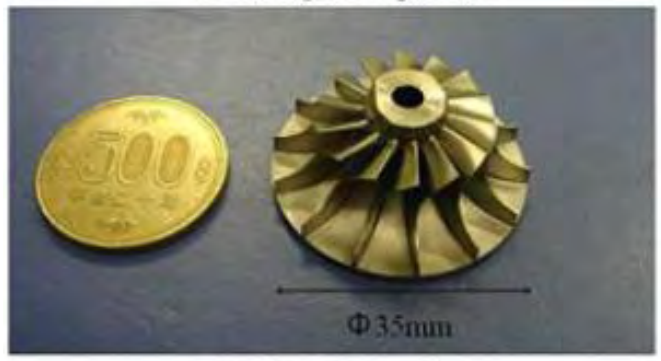

Radial inflow turbine

Figure 1.8. Centrifugal compressor's impeller and radial turbine designed and operated at the TITech test rig [29].

\subsubsection{Commercial s- $\mathrm{CO}_{2}$ Power Cycles}

The first commercial $8 \mathrm{MWe}$ closed s- $\mathrm{CO}_{2}$ Brayton cycle heat engine EPS100, developed by Echogen Energy Systems, was entered to the market in 2014. It turns waste heat from various industrial processes to electricity and operates at relatively low temperatures. A recuperated closed condensing Brayton cycle with multiple stages of recuperation and heat extraction from the primary heat source is employed by EPS100. The EPS100 uses two separate turbines. One, the 'drive turbine' is connected directly to the compressor, while the other 'power turbine' is coupled to a generator for power generation. The power turbine operates at a constant speed. The turbo-compressor speed can be varied independently over a wide range to maintain the optimal flow rate for the fluid loop for the given heat source and coolant conditions. Figure 1.9 shows a simplified cycle layout of the EPS100. The heat energy of the exhaust stream from industrial processes or gas turbines is recovered through a waste heat exchanger $\left(\mathrm{S}-\mathrm{CO}_{2}\right.$ heater $)$ by heating a flow of compressed S-CO $-\mathrm{CO}_{2}$. Downstream of $\mathrm{S}-\mathrm{CO}_{2}$ heater, the heated $\mathrm{S}-\mathrm{CO}_{2}$ flow is split into two main streams. Approximately two-thirds of the flow is directed to the power turbine, while the remainder is directed to the drive turbine that provides the shaft power for the main $\mathrm{S}-\mathrm{CO}_{2}$ compressor. The $\mathrm{S}-\mathrm{CO}_{2}$ streams exiting from the power turbine and drive turbine pass through recuperators to preheat the $\mathrm{CO} 2$ stream from the main compressor before being cooled, compressed and then sent to the $\mathrm{S}-\mathrm{CO}_{2}$ heater to complete the cycle. The power turbine has a single- 
stage radial design. The recuperators and $\mathrm{CO}_{2}$ coolers (condenser) are all of the PCHE type, while the $\mathrm{S}-\mathrm{CO}_{2}$ heater has a shell and finned tube design [41].

Echogen can now provide standard heat engines Scalable from 1 to $9 \mathrm{MWe}$. Based on the EPS100 system, Echogen has also completed a conceptual design of a 10-MWe S-CO $\mathrm{CO}_{2}$ test facility for the US DOE Nuclear Energy Group (Figure 1.9). Echogen is currently working with EPRI to develop integrated solutions for coalfired power plants using $\mathrm{S}-\mathrm{CO}_{2}$ power cycles as part of an ongoing US DOEfunded project. Several developers have developed conceptual designs of utilityscale coal-based closed $\mathrm{S}_{-} \mathrm{CO}_{2}$ cycle power plant including designs for key components such as boilers, heat exchangers and compressors. However, the viability of these designs needs to be tested and validated.

Moreover, Allam Cycle (Figure 1.10) is a power cycle that utilizes a recirculating $\mathrm{CO}_{2}$ working fluid in a high-pressure, low-pressure-ratio and highly recuperated Brayton cycle [30]. Since 2016, a power plant using Allam Cycle produces $25 \mathrm{MW}$ of electricity (50 MW of thermal load), while a $300 \mathrm{MW}$ version of the plant has been already designed and is going to begin in 2021 .

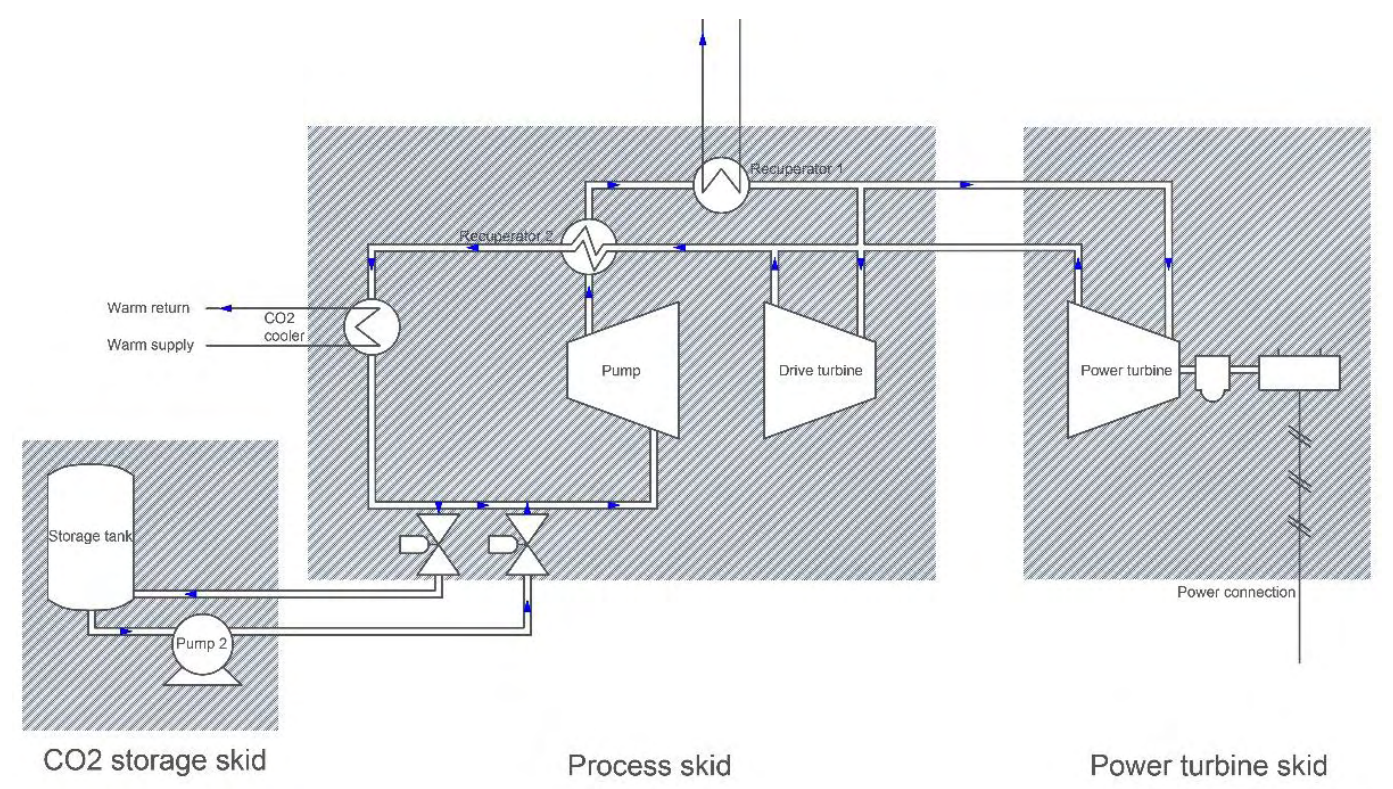

Figure 1.9. Process flow diagram of the EPS100 test facility [31]. 


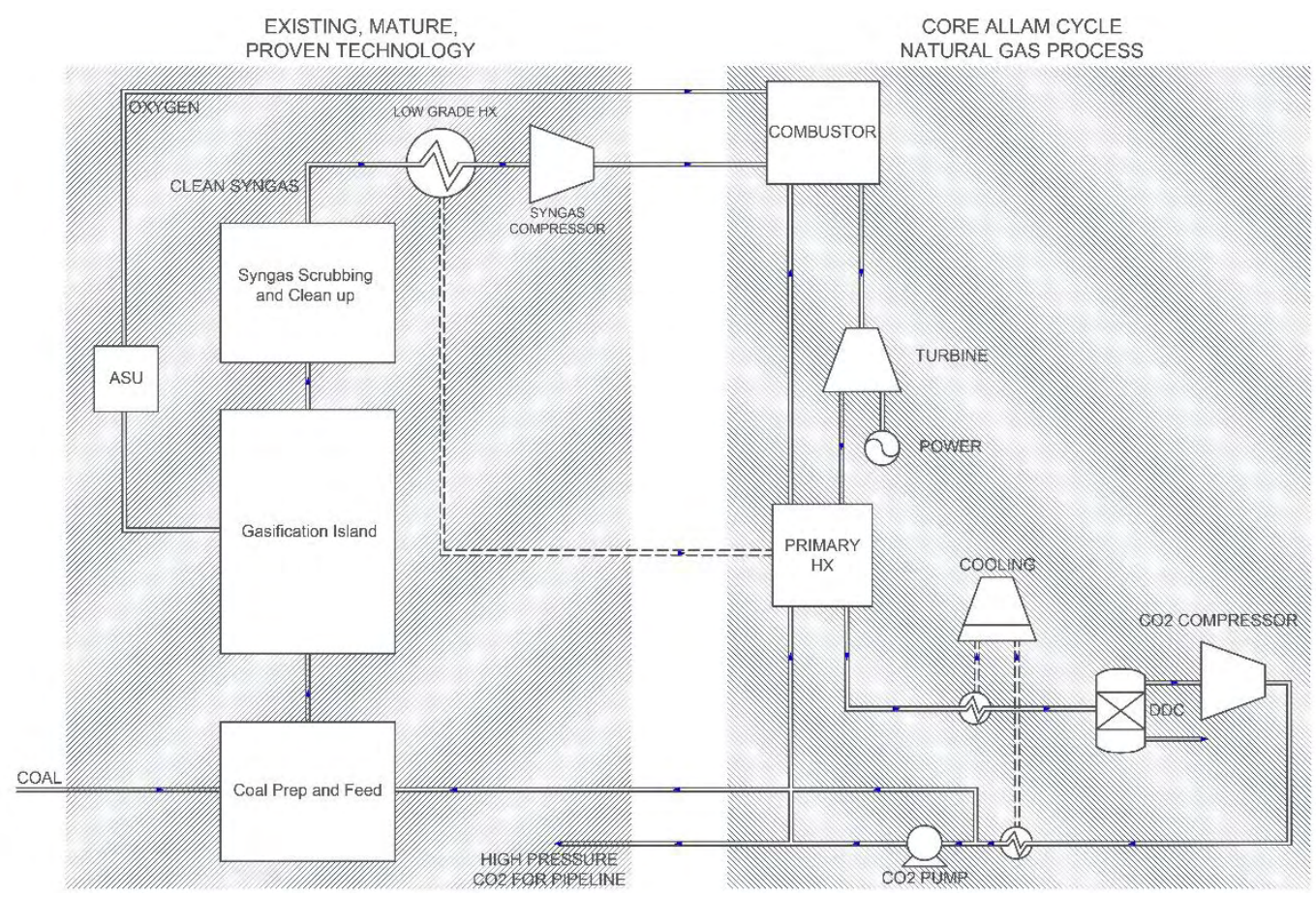

Figure 1.10. General arrangement of the coal Allam cycle for s- $\mathrm{CO}_{2}[30]$.

\subsection{Motivation}

The supercritical $\mathrm{CO}_{2}$ Brayton cycle is a promising alternative to the ideal gas (helium or air) Brayton cycles and the traditional water/steam Rankine cycle for power generation with nuclear or solar thermal sources. Also, it is very convenient for compact installations fired by natural gas. The $\mathrm{s}-\mathrm{CO}_{2}$ Brayton cycle has the following advantages: Carbon dioxide is less corrosive than water/steam, thus reducing material requirements at high temperatures. The $\mathrm{s}-\mathrm{CO}_{2}$ Brayton cycle can reach a higher cycle temperature, thus improving the thermal efficiency. Compared with the ideal gas Brayton cycles, the s- $\mathrm{CO}_{2}$ Brayton cycle has less compression work, thus higher thermal efficiency, while compared with the conventional power plants, the s- $\mathrm{CO}_{2}$ Brayton cycle requires significantly smaller turbines and heat exchangers.

Due to the above-mentioned advantages, research on s- $\mathrm{CO}_{2}$ power cycles is continuously increasing, focusing on thermodynamic cycle improvements as well as on the design optimization of critical components. As regards components optimization, a significant part of the work published in the literature concentrates on the heat exchangers (high and low-temperature recuperators) and the compressor. The heat exchangers constitute a crucial component and minimization 
of exergy losses must be effected with acceptable pressure drop, in order to improve cycle's efficiency.

On the other hand, compressors operate in a region around the critical point with a high density working fluid maximizing the compression work. However, the compressor needs to be carefully designed, because the acceleration of the flow could lead to two-phase flow in the compression stage, resulting in the decrease of its performance. Furthermore, the compressor blades are subjected to high stress levels and the bearing selection and performance are critical for efficient and reliable operation of compressor and turbine. Most research studies are based on standard compressor designs for conventional fluids.

The aim of this study is to investigate the performance of two critical cycle components, namely, the compressor and high temperature recuperator. The present work examines the thermal-hydraulic performance of printed-circuit heat exchangers in the cycle. The possible occurrence of two-phase flow may affect the compressor operational range. The real gas approximation employed for $\mathrm{S}-\mathrm{CO}_{2}$ is also studied because it influences the flow behavior, especially around the critical point of carbon dioxide, and determines under which circumstances the condensation could appear.

The outcomes of this study, are intended to be used as a reference regarding the design of printed circuit heat exchangers used as recuperators and their impact on cycle efficiency, and the performance of a centrifugal compressor stage for an operational range in applications with supercritical carbon dioxide.

\subsection{Goals and Objectives}

The goal of this thesis is to investigate the performance of printed-circuit heatexchangers and compressors as components of a recompression supercritical $\mathrm{CO}_{2}$

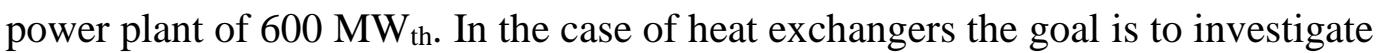
the thermal-hydraulic performance and examine how it affects the cycle efficiency (Figure 1.11), while for the compressors the goal is to investigate how the real gas effects influence the condensation on compressor and how the tip clearance gap impacts on the compressor performance (Figure 1.12).

In summary the methodology applied in this work in order to meet the objectives are to:

- Perform a thermodynamic analysis of the recompression s- $\mathrm{CO}_{2}$ Brayton cycle in order to study the relative importance of system's components on cycle performance.

- Perform an exergy analysis to find out the components that produce the maximum irreversibilities. 
- Design and model a printed-circuit heat exchanger (PCHE) in order to study its thermal-hydraulic performance and optimize it for the specific plant.

- Validate the heat exchanger performance models employed against available test results on a PCHE of similar technology.

- Investigate how the designed PCHE affects the cycle efficiency.

- Design a centrifugal compressor for the $\mathrm{s}-\mathrm{CO}_{2}$ power cycle taking into consideration specialized loss models.

- Quantify the impact of real gas effects on numerical simulation of centrifugal compressor.

- Examine possible condensation in the compressor stage for alternative design options, such as varying tip clearance gap.

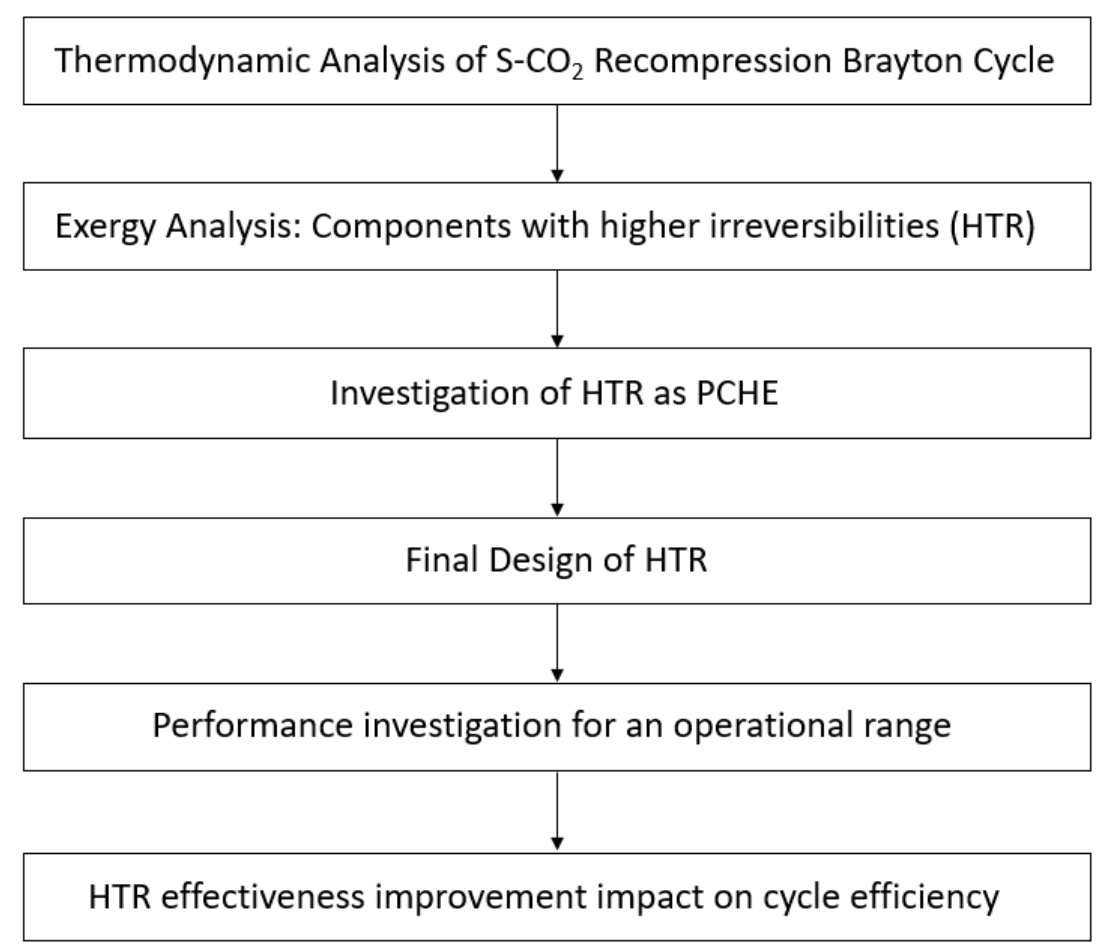

Figure 1.11. Thesis roadmap for the heat exchanger design and study. 


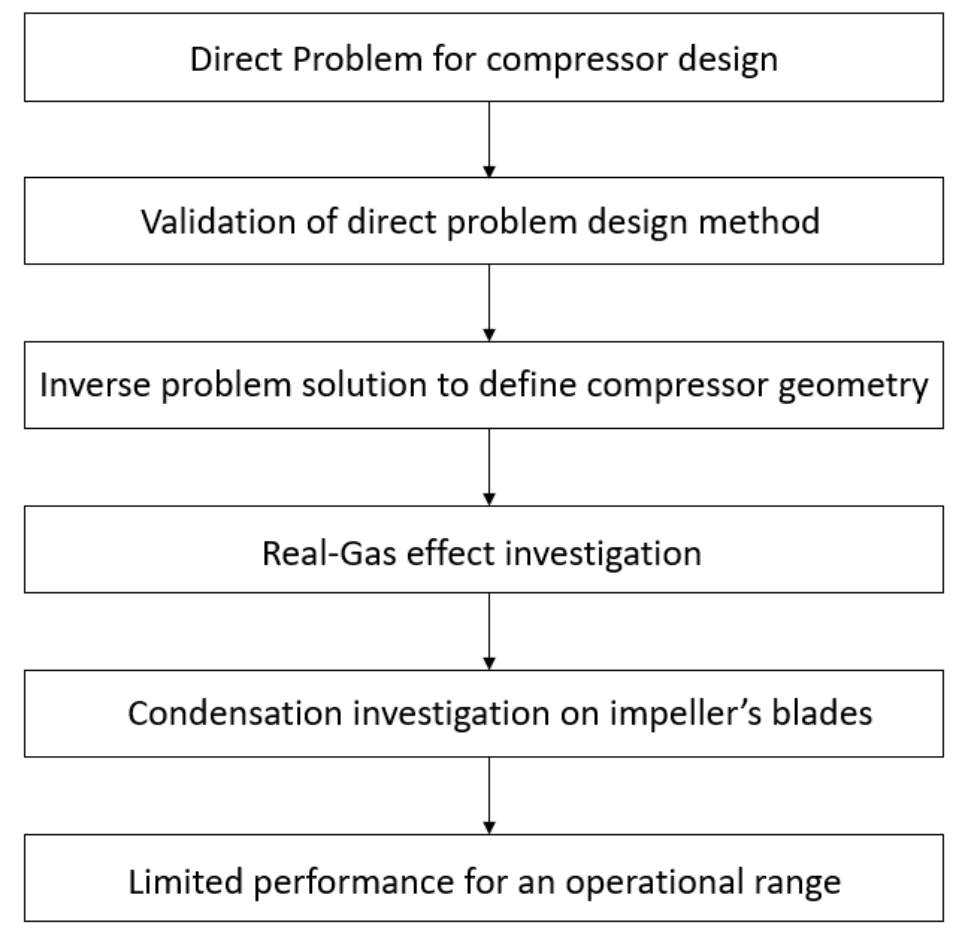

Figure 1.12. Roadmap for the compressor design and investigation.

\subsection{Contributions to Science and Technology}

The most important results from this thesis that can be considered as novel are the following:

- The cycle's efficiency is improved by the optimized design of a printed-circuit heat exchanger (HTR), supported by numerical models, resulting in improved heat exchanger effectiveness (higher than 99\%), with acceptable pressure drop $(<1 \%)$.

- The real gas effects influences the simulation results. The study showed that a look-up table with high resolution close to critical point can predict with higher accuracy the condensation effect.

- The condensation investigation resulted in a limited operational range of compressor performance map in order to ensure supercritical flow. The range is limited to a $30 \%$ of the initial range for low rotational speed to a $25 \%$ for higher rotational speeds. 


\subsection{Organization of the Thesis}

The thesis is organized in nine chapters, including the Introduction (Chapter 1). In the second chapter a literature review takes place, concerning the printed-circuit heat exchangers and the compressor design, which constitute the two main branches that this thesis deals with.

In Chapter 3 a thermodynamic analysis of the recompression Brayton cycle with $\mathrm{s}-\mathrm{CO}_{2}$ is presented and the impact of significant parameters on the cycle efficiency is discussed, while an exergy analysis is performed too. Chapter 4 and Chapter 5 contain the methods employed for the design and study of the printed-circuit heat exchangers and centrifugal compressors respectively. In the fourth chapter, the one-dimensional model developed for printed-circuit heat exchangers is analyzed, including the steady-state and dynamic models, the numerical model for the threedimensional analysis is discussed and the employed experimental method is presented. Chapter 5 presents the design method developed for the centrifugal compressor analysis, incorporating the direct and inverse problem and the loss models used. Moreover, the numerical method used for the three-dimensional analysis and the real gas effect on the numerical solution are explained.

The results of the presented methods are presented in Chapter 6 and 7 for printed-circuit heat exchangers and centrifugal compressors respectively. Finally, in Chapter 8 the conclusions of the thesis and future work suggestions are discussed, while Chapter 9 includes the references of the thesis. 


\section{Chapter 2}

\section{Literature Survey}

Supercritical carbon dioxide cycles constitute a relatively new technology for power generation. Consequently, many components of the power cycle need further study in order to reach a mature level in the commercialization of this technology. The design of the heat exchanger and the compression system presents significant challenges in the way of making the operation efficient, safe and cost effective. This chapter presents a detailed literature review on the research done regarding printed-circuit heat exchangers used as high and low temperature recuperators in supercritical carbon dioxide power cycles, as well as the compressor design and performance investigation in order to ensure an operation above the critical point.

\subsection{Printed-Circuit Heat Exchangers}

A large amount of heat is recovered in recuperators using supercritical carbon dioxide in order to increase the thermal efficiency of the thermodynamic cycle. Hence, the high effectiveness of heat exchangers is a critical design consideration. Moreover, the use of conventional heat exchangers would increase the capital cost of large establishments. In consequence, compact heat exchangers such as printedcircuit heat exchangers (PCHE), have been introduced in market and can be applied in s- $\mathrm{CO}_{2}$ applications. These heat exchangers are made of high-strength materials in order to operate safely in high pressure. PCHE consists of many plates bonded together with channels manufactured by chemical etching. Their high surface area per unit volume allows a closer temperature approach in a more compact block compared to an equivalent Shell and Tube heat exchanger. Moreover, a wide range of materials can be used for PCHE manufacture, including high grade alloys which are useful for higher temperature or pressure applications [32]. Figure 2.1 presents the way that the hot and cold plates are joined together. 

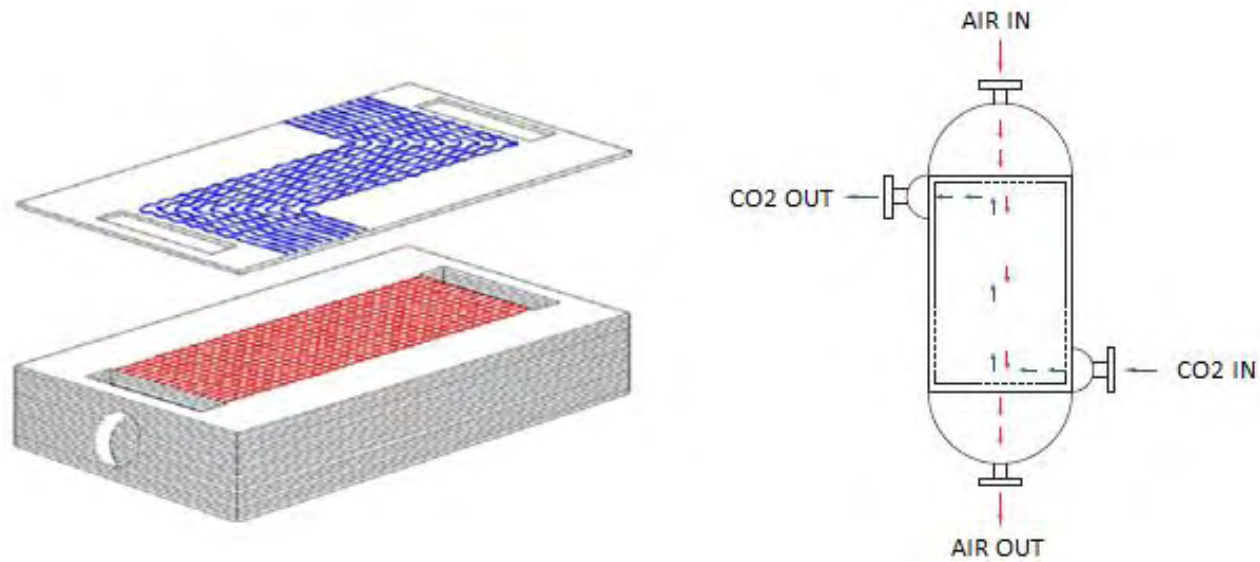

Figure 2.1. Structure of the PCHE's hot and cold plates [32].

The channels are semicircular with diameters between 0.8 and $2.2 \mathrm{~mm}$. The channels can be straight, wavy (Figure 2.3), S-shaped fin or airfoil-finned channel (Figure 2.2) depending on the process requirements. The wavy design constitutes the most common practice as it enhances the heat transfer and decreases the component length. Many studies have been carried out to investigate the optimum design for specific applications, having the minimum pressure drop and the maximum heat transfer [33-37].

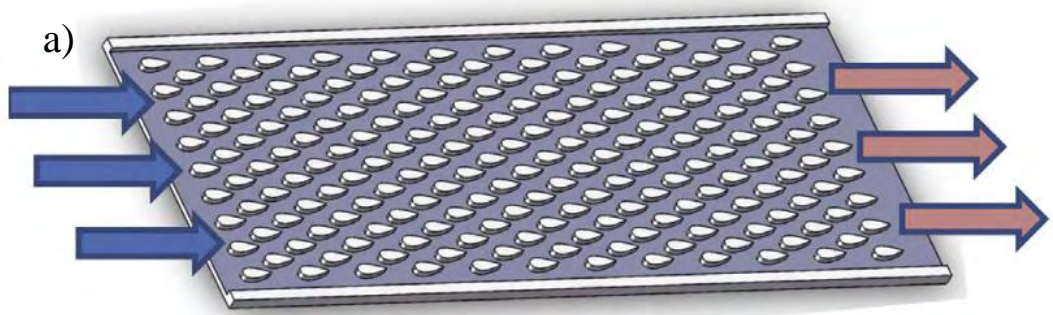

b)
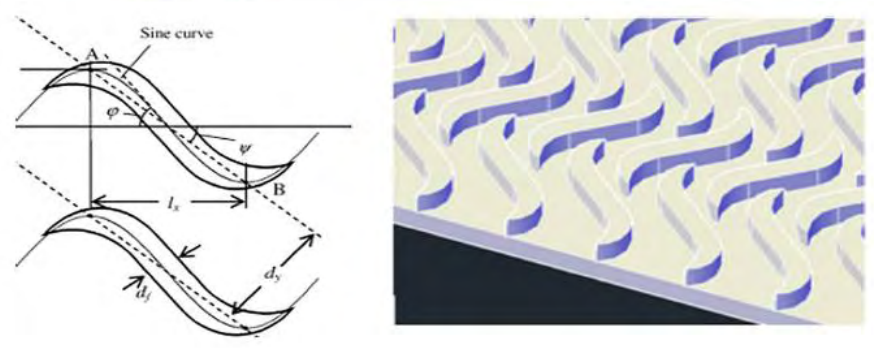

Figure 2.2. (a) Airfoil finned and (b) S-shaped finned channels for PCHE $[33,35]$. 


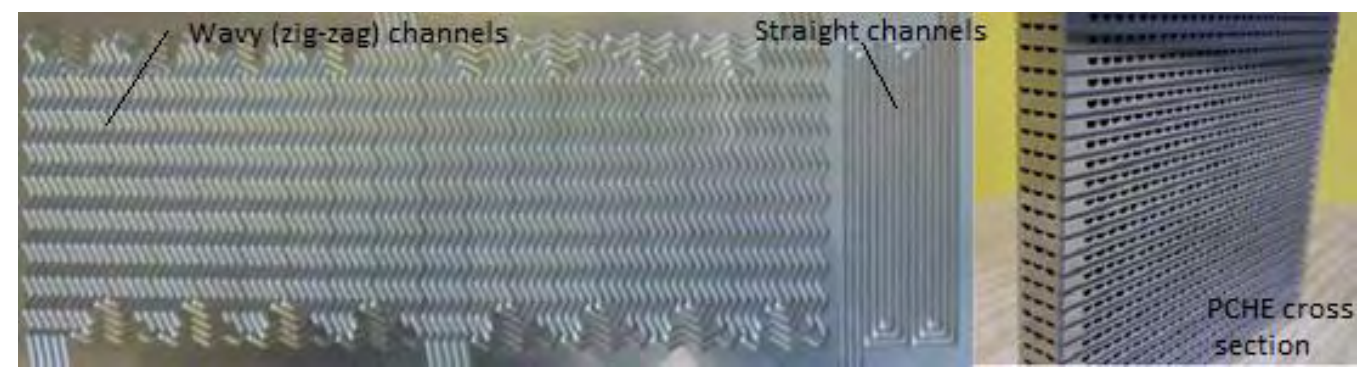

Figure 2.3. Plate with straight and wavy channels and cross section of the PCHE $[31,37]$.

The angle increment for the wavy channels has a wide range, as the etching process offers this possibility. Moreover, the plates' arrangement in the heat exchanger's block present two different configurations, either parallel or offset, as presented in Figure 2.4. In the parallel configuration the plates are stacked in a way that the hot and cold channels are aligned, while for the offset configuration there is a shift between them. Each design choice presents a different thermal-hydraulic performance, covering a range of applications.

The printed-circuit heat exchangers' weight varies according to the application, reaching up to 60 tones, while the maximum design pressure and temperature can reach up to $65 \mathrm{MPa}$ and $1160 \mathrm{~K}$, assuring the appropriate mechanical integrity. Heatric (UK), a manufacturing company over 30 years old, has sold more than 1000 units mainly in the oil and gas industry. The design considerations, material selection, configuration options and manufacturing methods of PCHEs for potential application in the nuclear power industry have been discussed in detail by engineers at Heatric $[32,38,39]$. The following table (Table 2.1) presents the main design characteristics for the Heatric printed-circuit heat exchangers.
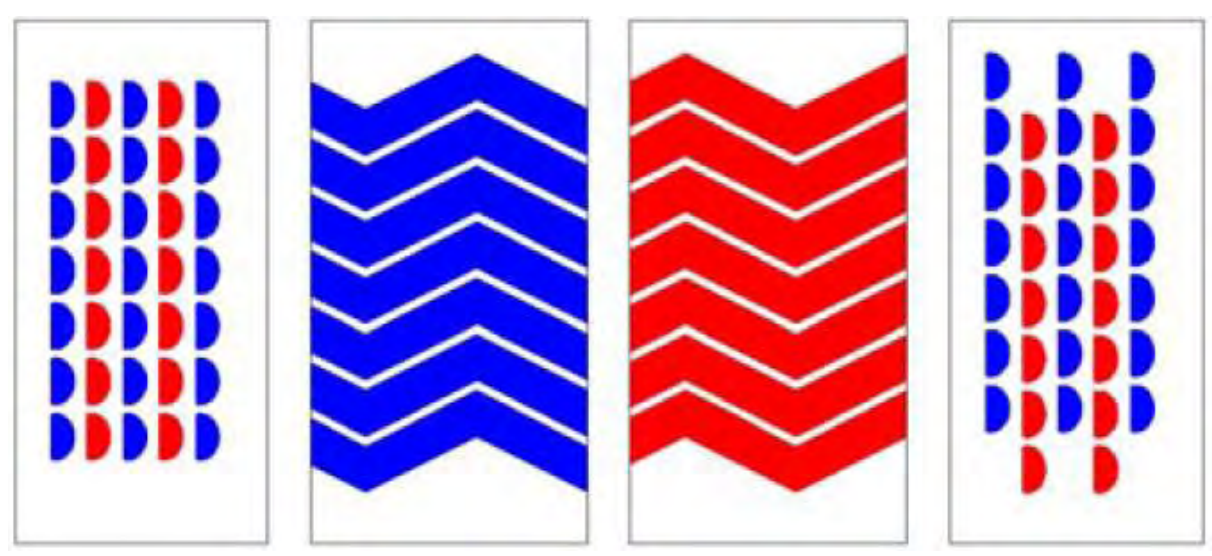

Figure 2.4. Parallel and offset configuration of plates in the PCHE [32]. 
Table 2.1. PCHE design characteristics [40].

\begin{tabular}{lcl}
\hline \multicolumn{1}{c}{ Characteristic } & & \multicolumn{1}{c}{ Value } \\
\hline Unit weight & $\mathrm{kg}$ & $1-60000$ \\
Maximum design pressure & $\mathrm{bar}$ & 650 \\
Design temperature range & $\mathrm{K}$ & $2-1160$ \\
Maximum surface area & $\mathrm{m}^{2}$ & 10000 \\
Area/unit volume & $\mathrm{m}^{2} / \mathrm{m}^{3}$ & $1300(100$ bar $)$ \\
& $\mathrm{m}^{2} / \mathrm{m}^{3}$ & $650(500$ bar $)$ \\
Minimum $\Delta \mathrm{T}$ & $\mathrm{K}$ & 1 (typically $3-5)$ \\
Effectiveness & - & up to $98 \%$ \\
Overall HTC & $\mathrm{W} / \mathrm{m}^{2} \mathrm{~K}$ & LP gas cooler $500-1000$ \\
& $\mathrm{~W} / \mathrm{m}^{2} \mathrm{~K}$ & HP gas cooler $1000-4000$ \\
Plate thickness & $\mathrm{mm}$ & $0.5-5.0$ \\
Passage width & $\mathrm{mm}$ & $0.5-5.0$ \\
Reynolds number & - & Gases: $10^{3}-10^{5}$ \\
& - & Liquids: $10-5000$ \\
\hline
\end{tabular}

The effectiveness of the PCHE can reach up to $98 \%$, presenting a minimum pinch of 1 degree (typically 3-5 degrees). The PCHE, operating with supercritical $\mathrm{CO}_{2}$ close to critical point, are often affected by a pinch-point problem on both the hot and the cold side. This means that the temperature difference approach does not provide a reliable heat transfer between the hot and the cold side, but the temperature gradient could also have a negative value. The pinch point is typically located at the inlet or the outlet of a heat exchanger if the specific heat capacity of fluids inside the heat exchanger is constant. However, when the specific heat is changing during the heat transfer process, the pinch point can be located inside a heat exchanger (Figure 2.5). The pinch point is observed when the heat capacity of the hot and cold streams are equal for two different pressure levels. Specifically in the case of carbon dioxide that its properties vary in a non-linear and intense way near the critical point, there is a high probability of presenting the same heat capacity at the hot and cold side of the heat exchanger, leading to a pinch-point problem. Pinch point location also depends on geometry of heat exchanger and physical properties of fluid. The occurrence of a pinch point is a significant issue for heat exchanger design, as it makes its performance unstable leading in a low efficiency and probably over-sized heat exchanger. Therefore, a common practice to estimate the PCHE performance considering a fixed pinch $(4-5 \mathrm{~K})$ at the exit of the flow. 


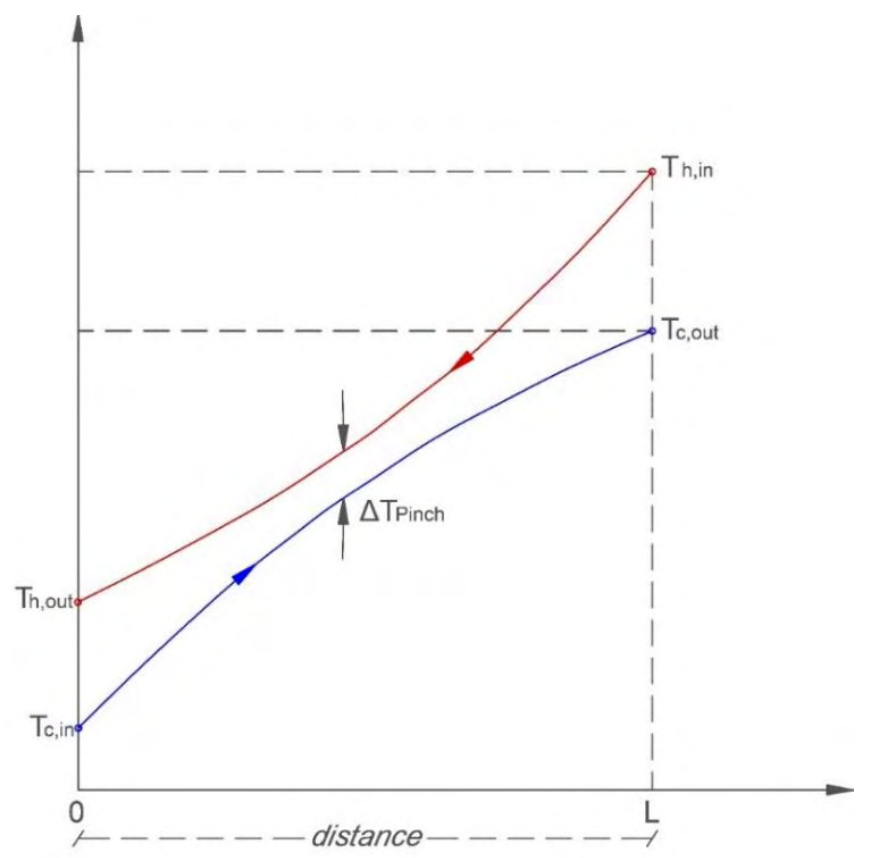

Figure 2.5. Pinch-point problem for heat exchanger.

The design of the PCHE is a procedure that has to take many parameters into account. The pressure drop and the heat transfer rate can significantly affect the cycle performance, while the presence of a pinch-point problem may lead to a reduced cycle performance. This the reason that so much research is focused on the thermal-hydraulic parameter of the PCHE for s- $\mathrm{CO}_{2}$ power cycle, using both numerical models and experimental test facilities. Wang (2003) [41] and Dostal (2004) [3] from Massachusetts Institute of Technology carried out the first studies on PCHE, regarding their application on nuclear reactors. In the next years, many studies have been conducted on PCHEs' design and steady-state and dynamic performance [42], focusing mainly on the numerical and experimental investigation of the thermal-hydraulic performance and the optimization of their geometry.

\subsubsection{Steady state thermal-hydraulic performance of PCHE}

Nikitin et al. (2006) [43] examined the heat transfer and pressure drop of a 3 $\mathrm{kW}$ PCHE employing zigzag channels and operating with $\mathrm{S}_{-} \mathrm{CO}_{2}$ on both its hot and cold sides. It was found that the overall heat transfer coefficients varied at 300 to $650 \mathrm{~W} /\left(\mathrm{m}^{2} \mathrm{~K}\right)$. Tsukuzi et al. in 2007 [44] investigated S-shaped finned channels of a PCHE, concluding in a five-times reduced pressure drop in comparison with a PCHE with zigzag channels, for similar heat transfer performance. Ngo et al. 
(2007) [45] investigated the heat transfer and the pressure drop of a PCHE with zigzag and $\mathrm{S}$-shape finned channels for $\mathrm{S}-\mathrm{CO}_{2}$, employing both three-dimensional computational fluid dynamics (CFD) code and experiments. The results showed that zigzag channels presented a $24-34 \%$ higher Nusselt number than S-shape finned channels, while their pressure drop was about 4-5 times higher depending on the Reynolds number. In 2008, Kim et al. [34] presented a numerical three dimensional analysis on zigzag channels of PCHE and proposed a PCHE with airfoil-finned channels, obtaining a pressure drop equals $8 \%$ of the pressure drop that the zigzag channels present for the same heat transfer performance. Kim also conducted experimental studies on PCHEs with working helium-helium and helium-water on hot and cold sides [46, 47], proposing correlations for Nusselt number and Fanning factor for zigzag channels. Mylavarapu et al. [48, 49] designed and constructed a test facility with PCHE employing high-temperature helium at temperatures and pressures up to $800{ }^{\circ} \mathrm{C}$ and $3.0 \mathrm{MPa}$, with helium mass flow rates from 15 to $49 \mathrm{~kg} / \mathrm{h}$. Two similar straight-channel PCHEs were fabricated using Alloy 617 plates in order to withstand creep and their thermal-hydraulic performance was investigated. In 2012, Kruizenga et al. [50] investigated the heat transfer of supercritical $\mathrm{CO}_{2}$ in horizontal semicircular channels, showing that the existing correlations over-predict heat transfer near the critical point, and a new correlation was proposed. In 2013, Figley et al. [51] investigated the laminar and laminar-to-turbulence transition behavior of the channels manufactured in the previous study [48]. The numerical results showed that the transition is observed at Reynolds numbers of 2300 for the circular and 3100 semicircular channels, while the heat transfer and pressure drop characteristics were also investigated. Yoon et al. [52] from Idaho National Laboratory (INL) presented general methods for thermal design and cost estimation in order to determine the size, the thermalhydraulic characteristics and the cost of a crossflow PCHE. The effectiveness calculated by the developed code showed a good agreement with the results obtained with the $\varepsilon$-NTU method.

Baik et al. (2015) [53] investigated experimentally the thermal-hydraulic performance of a PCHE used as precooler in a supercritical $\mathrm{CO}_{2}$ Brayton cycle, and the results were compared with the performance of a conventional shell-andtube heat exchanger, resulting in a lower pressure drop for the PCHE. Schmitt et al. [54] conducted a numerical design study of a PCHE for a $100 \mathrm{MW} \mathrm{s- \textrm {CO } _ { 2 }}$ Brayton cycle, focusing on the Reynolds number, the Nusselt number and the fluid temperature along the high and low temperature recuperator and resulting in an overall heat transfer coefficient of $850 \mathrm{~W} /\left(\mathrm{m}^{2} \mathrm{~K}\right)$ and $1097 \mathrm{~W} /\left(\mathrm{m}^{2} \mathrm{~K}\right)$ respectively. Khan et al. (2015) [55] studied numerically wavy (zigzag) channels using threedimensional numerical model(CFD), resulting in thermal-hydraulic characteristics and incorporating the incline angles in this study in order to investigate the 
secondary flow regimes. Aneesh et al. (2106) [56] also carried out a numerical study on straight-channel of PCHE with working helium, investigating the effects of operating conditions, the modification of the flow pattern and hemispherical dimples inside the channels on thermal-hydraulic performance. Chen et al. (2016) [57] presented the fabrication procedure of a counter-flow PCHE with zigzag channels that could be used in high-temperature and high-pressure conditions and performed an investigation of the pressure drop and the heat transfer coefficient for the fabricated PCHE in the transitional flow regime. Meshram et al. [58] developed an one-dimensional tool to investigate Reynolds and Nusselt number and the temperature variation in a PCHE with straight and zigzag channels. Moreover, Guo [59] presented a segmental analysis of a printed-circuit heat exchanger, in order to find out the heat transfer rate and the effectiveness. Kim et al. (2016) [60] used a computational fluid dynamics tool in order to explore the validity of existing correlations for Nusselt number and friction factors and proposed a new correlation derived by computational fluid dynamics (CFD) model for a Reynolds number of $2 \times 10^{3}-5.8 \times 10^{4}$.

Yoon et al. (2017) [61] conducted a detailed numerical study of the friction factor and heat transfer for laminar flow in semi-circular zigzag channel of PCHE with various geometrical parameters. General explicit correlations were developed for both the friction factor and Nusselt number as a function of geometrical parameters, Reynolds and Prandtl numbers based on three-dimensional computational results. The study indicated that the friction factor for zigzag channels in PCHEs was mainly influenced by zigzag channel geometry while the Nusselt number was influenced by the overall heat exchanger design, including the plenum sections. Also, Baik et al. [62] investigated numerically and experimentally the flow phenomena of a $\mathrm{CO}_{2}$-water PCHE for a s- $\mathrm{CO}_{2}$ Brayton cycle, resulting in friction factor and heat transfer correlations in a Reynolds number range of $15 \times 10^{3}$ $10^{6}$ and Prandtl number range of 2-33. Moreover, Chen et al. [36] presented a comprehensive study on the airfoil-finned channels of a PCHE, while Chu et al. (2017) [63] investigated experimentally the straight channels' heat transfer characteristics of a s- $\mathrm{CO}_{2}$-water $\mathrm{PCHE}$, investigating the region that carbon dioxide approximates the critical point.

In 2018, Aneesh et al. [64] investigated the effects of different wavy channels configuration on the thermal-hydraulic performance of a PCHE Alloy 617 with working helium on both sides, reporting that trapezoidal PCHE model presents the highest heat transfer rate, $41 \%$ higher than straight channels, but with a pressure drop penalty. Cui et al. [37] and Ding et al. [33] (2018) investigate alternatives geometries in order to improve the heat transfer effects in airfoil-finned channels of $\mathrm{PCHE}$ for $\mathrm{s}-\mathrm{CO}_{2}$ applications. 
As a general remark, the above mentioned research works study the extents of applicability of the state of the art PCHE manufacturing technology. On the other hand, this technology is constantly improving and the advances of the printed circuit technology allow improved design versions, especially regarding the curvatures of the wavy channels and the improved heat transfer rates.

\subsubsection{Dynamic performance of PCHE}

Apart from the steady-state heat transfer and pressure drop investigation, the understanding of the dynamic behavior is significant as the varying thermal load can result in design options that could alter the PCHE design for safety reasons and performance improvement. Any change of the load in a supercritical $\mathrm{CO}_{2}$ power plant would lead to transient phenomena in all the components of the system including heat exchangers. Although the steady-state thermal-hydraulic performance of PCHEs has been investigated thoroughly, limited research on transient behavior of PCHEs, numerical or experimental, has been conducted. In literature, Sharifi [65] investigated the dynamic performance of plate heat exchangers in 1995, but after the s- $\mathrm{CO}_{2}$ cycle revival Carstens (2007) was the first [66] that developed a model to solve the energy, momentum and continuity equations in order to model the transient effects for a PCHE of a s- $\mathrm{CO}_{2}$ cycle. A year later, Pra et al. [67] published some transient test cases in order to study the behavior of a PCHE, without developing a transient model to validate it. Since then, according to the author's knowledge, no research studies have been published on PCHE's dynamic performance, until the investigation of Chen et al. (2016) [68]. The study validates results derived from a dynamic model with experimental results for straight-channel PCHE with helium working fluid, resulting in good accordance between models and experiments for various test cases. In 2018, the same research group published a study presenting numerical and experimental results of the steady-state and dynamic performance of a PCHE with zigzag channels [69]. Furthermore, Jiang et al. [70] and Park et al. [71] presented analyses of the dynamic behavior of a PCHE, comparing experimental data with transient models. 


\subsection{The Centrifugal Compressor}

The compressor is one of the most important components of the cycle, as the development of a compressor that can stably operate over a wide range helps ensures a robust operation of the engine. The centrifugal compressor consists of four main elements, namely inducer, impeller, diffuser and volute. The flow is pressurized as it passes through the impeller and the radial vanes of the diffuser. Qualitative structure of the centrifugal compressor is presented in Figure 2.6. A significant detail regarding the recompression $\mathrm{s}-\mathrm{CO}_{2}$ cycle is that it requires the inlet of the compressor to be close to the critical point in order to exploit the abrupt properties variation of carbon dioxide at this point, decrease the compression work and finally improve the cycle efficiency. The compression has to be completed by a reliable design, without allowing two-phase flow in the compressor because of the flow diffusion.

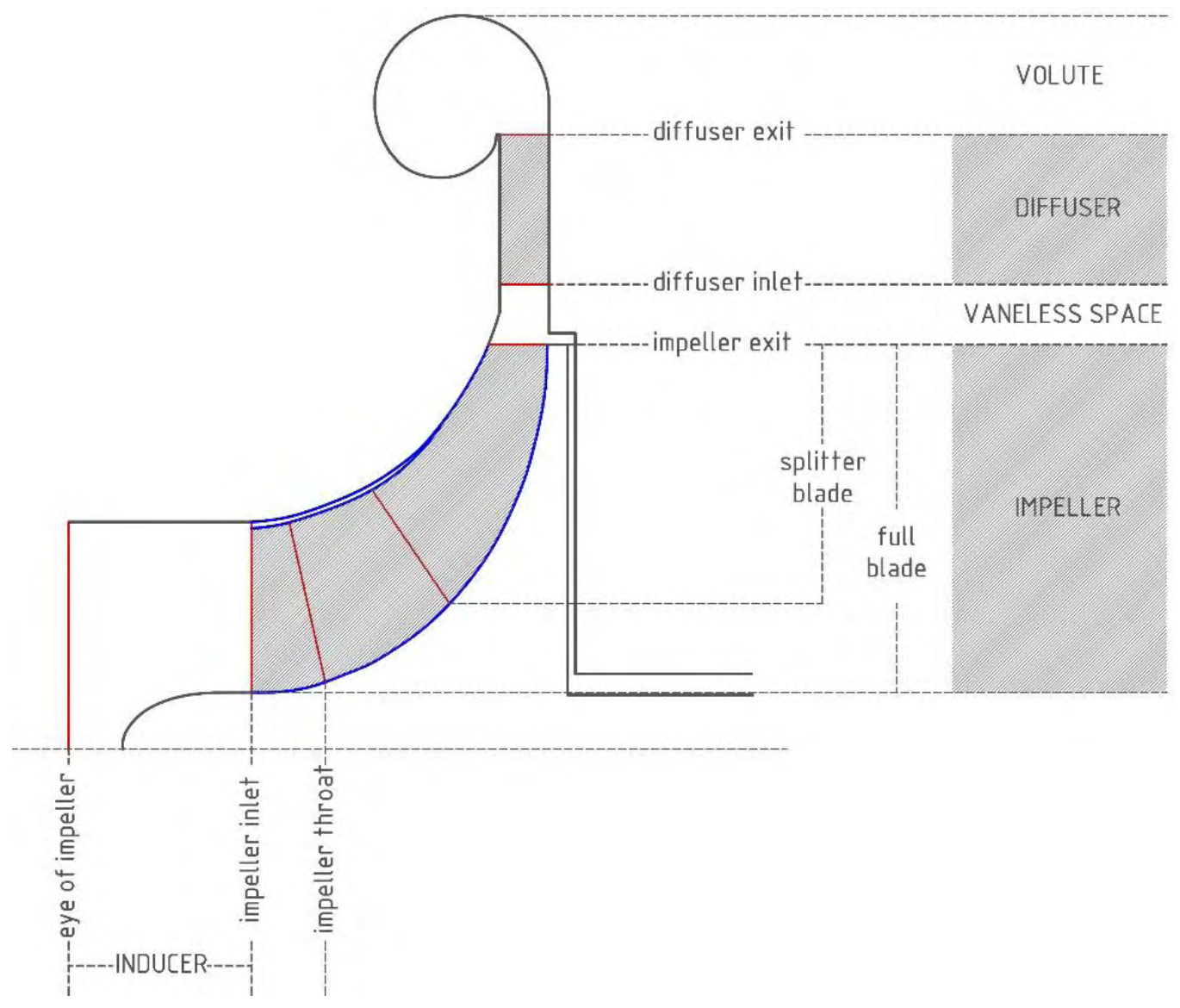

Figure 2.6. Configuration of a centrifugal compressor stage. 
Supercritical carbon dioxide power cycles demand a high nominal and acceptable off-design efficiency of the compressor, as the compressor's aerodynamic performance can decisively affect the whole system. The volute is not included at this study, as there is no available design for supercritical $\mathrm{CO}_{2}$ to compare with.

\subsubsection{Design consideration methods}

In order to design a compressor and study its performance, the understanding of the flow distribution among the blades is indispensable, as the unsteadiness of the flow can make the phenomena really complex. The analysis of the flow-field within the rotating blades is performed in a frame of reference that is stationary relative to the blades, where the flow is considered steady. The analysis of the velocity triangles and the flow parameters in the relative frame of reference is a basic part of the turbomachine's study.

Figure 2.7 presents the velocity triangles at the inlet and outlet of the impeller.

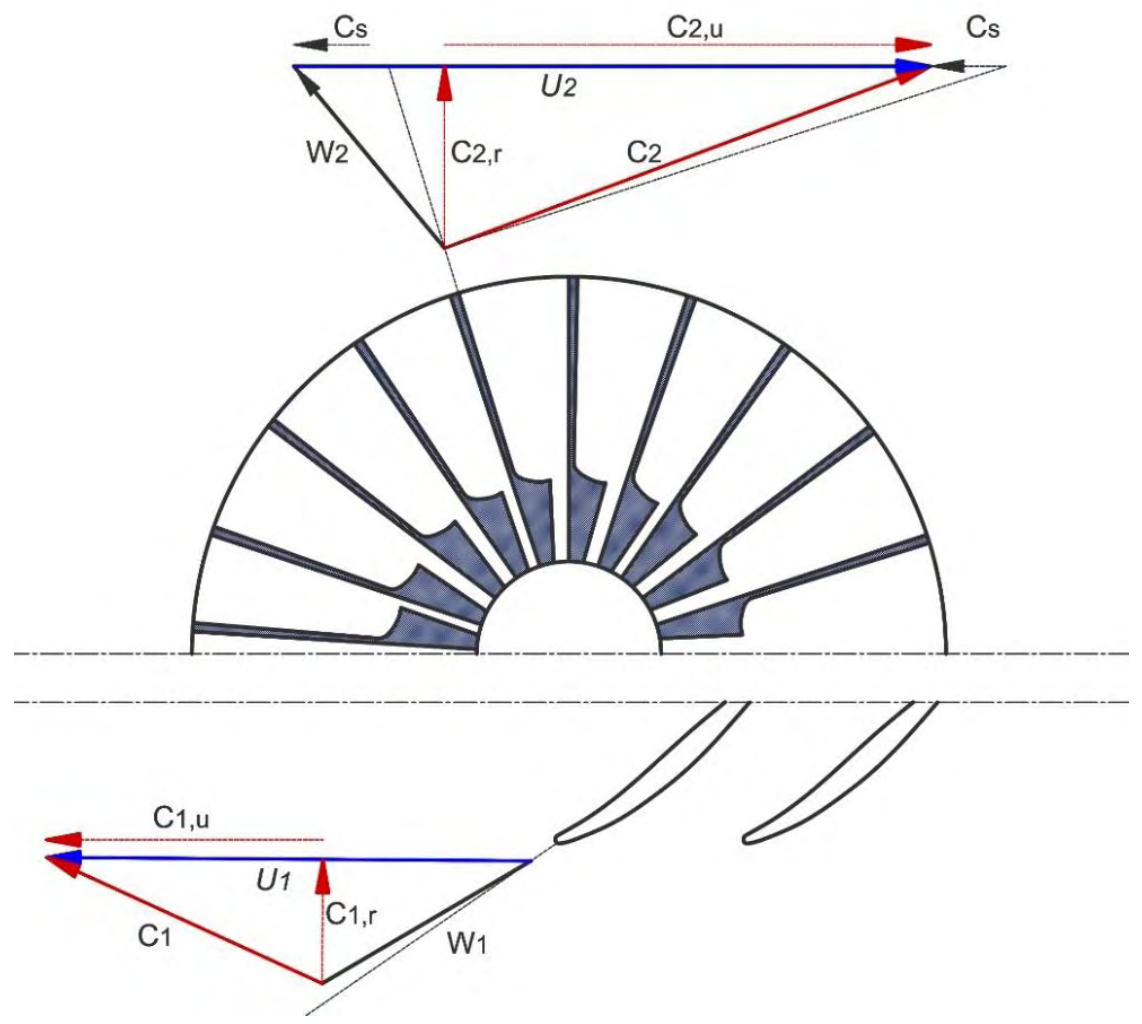

Figure 2.7. Velocity triangles at inlet and outlet of the impeller. 
The analysis is performed using three velocity components:

- Absolute velocity $\vec{c}$ : the flow velocity with reference to the stationary frame

- Circumferential blade velocity $\vec{U}$ : the tangential velocity of the rotating shaft, derived as $\vec{U}=\vec{\omega} \times \vec{r}$

- Relative velocity $\vec{w}$ : the relative velocity of the flow, derived as vector subtraction of the blade velocity $\vec{U}$ of the absolute velocity $\vec{c}$.

The work done in the compressor is derived by the Euler's equation that combines the aerodynamic characteristics of the flow with the thermodynamic:

$$
\dot{W}_{c}=h_{2}-h_{1}=U_{2} c_{\theta, 2}-U_{1} c_{\theta, 1}
$$

Different methodologies have been proposed for the centrifugal compressor's design. Although most of the design approaches have been developed for conventional working fluids (mainly air), they are also applied in the case of supercritical $\mathrm{CO}_{2}$.

The first studies on supercritical $\mathrm{CO}_{2}$ turbomachinery design used the preliminary design proposed by Balje (1981) [72] for conventional turbomachinery. Balje proposed a model that is based on the evaluation of displacement and momentum boundary layer thickness, incorporating only three loss mechanisms, profile, endwall and gas clearance losses. Moreover, Balje developed a general diagram for both types (axial and radial) of compressors and turbines, using the specific speed $\left(\mathrm{N}_{\mathrm{s}}\right)$ and the specific diameter $\left(\mathrm{D}_{\mathrm{s}}\right)$ (Figure 2.8). In general, centrifugal compressors are characterized by specific speed of 0.4-1.8 with optimum values 0.6-0.77 [25]. Wang [41] in 2003 designed an axial compressor for a $300 \mathrm{MWe}$ s- $\mathrm{CO}_{2}$ cycle (Dostal cycle), while Gong [73] in 2006 used a one-dimensional tool in order to design a single-stage centrifugal compressor for the same cycle At the moment the majority of the research interest is concentrated on the centrifugal compressors even for large scale power cycles, and many research groups focus on centrifugal rather than axial flow compressors. Gong and Fuller [25] proposed the Balje diagram as a reliable tool for centrifugal compressor's preliminary design.

A design methodology proposed by Whitfield and Baines (1990) [74] for both compressors and turbines, estimating the enthalpy drop as dimensionless energy loss instead of pressure loss. Therefore, the enthalpy loss through the compression process is estimated and the entropy change $(\Delta s)$ of the process can be computed, an approach also proposed by Denton (1993) [75]. Oh et al. (1997) [76] developed a similar loss model, estimating the enthalpy drop through the compressor in dimensions. 


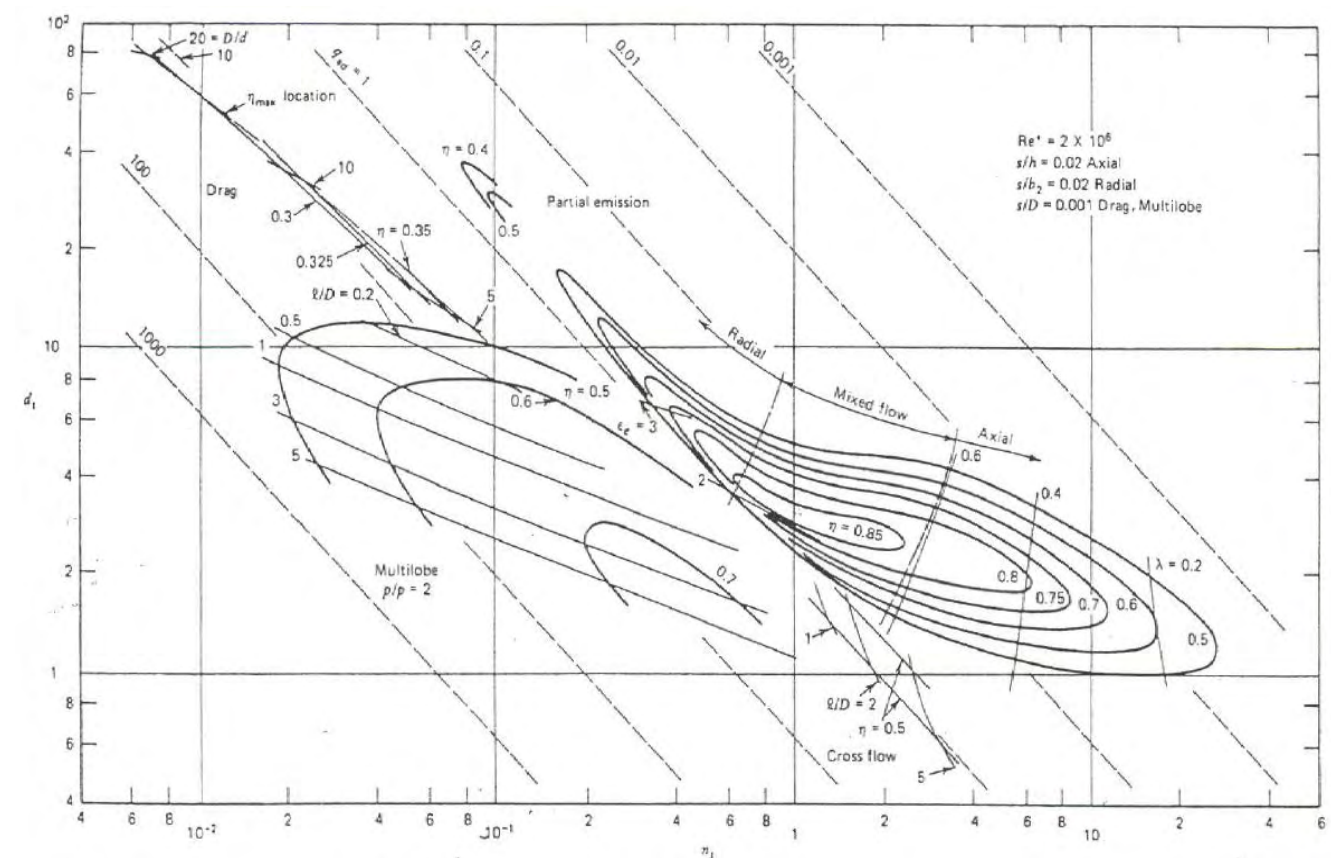

Figure 2.8. Balje diagram for compressors design [72].

Another design methodology was proposed by Japikse (1996) [77], focusing on the two-zone model, developed firstly by Dean-Senoo (1960), based on splitting the flow into an isentropic main flow (jet) and a non-isentropic distorted flow (wake) at the impeller. This method requires parameters that are defined experimentally for conventional turbomachines, but are inaccurate for $\mathrm{s}-\mathrm{CO}_{2}$ compressors since there is no adequate experimental data. Another methodology for radial turbomachinery design has been proposed by Aungier (2000) [78] and was also adopted by Monge (2014) [79]. This study is not based on two different zones, like the previous methods, but incorporates several loss coefficients for each of the compressor's elements, resulting in a total pressure loss coefficient. The current study for compressor design, also, uses the Aungier's approach combined with some modifications proposed by Monge. Table 2.2 concentrates all the losses incorporated in this study for each compressor's element.

Since then, research efforts on supercritical $\mathrm{CO}_{2}$ compressors have mainly focused on optimizing the compression process to reduce the power requirements and avoid the condensation of the supercritical carbon dioxide. Several variations of the conventional centrifugal compression system are discussed by Moore et al. [80]. To improve the efficiency of the process, a semi-isothermal compression is proposed using an intercooling between each centrifugal stage. This approach however, adds significant complexity to the system and leads to potential reliability risks. Moore et al. [81] have also presented an internally cooled centrifugal compressor, where a cooling jacket is embedded around the gas flow path. The 
experimental results presented for a single stage test rig showed that over $50 \%$ of the heat addition by the impeller can be removed. The feasibility of this concept in a multistage configuration at supercritical pressures is still questionable.

Moreover, much of research that takes place in research laboratories, such as Sandia National Laboratories in USA, Tokyo Technology Institute in Japan or KAIST in South Korea, operate experimental facilities and focus on the feasibility of the system and components investigation. The compressor operation in Sandia has led to a performance range [28] of the small scale centrifugal compressor, while Utamura et al. [29] from TITech presented an experimental study on centrifugal compressor of $30 \mathrm{~mm}$ diameter and designed for a rotational speed of $100 \mathrm{krpm}$. The windage losses were measured and the maximum work output was $206 \mathrm{~W}$. The experimental data were compared with calculations based on a meanline tool developed by Concepts ETI, showing good accordance.

A discussion on deriving the turbomachinery specifications from the cycle analysis is presented by Fuller at al. [25], while a two-dimensional model for the design of supercritical $\mathrm{CO}_{2}$ turbomachinery is proposed by Lee et al [16].

Table 2.2. Loss models adopted for centrifugal compressor design.

\begin{tabular}{ll}
\hline \hline \multicolumn{2}{c}{ Centrifugal Compressor Losses } \\
\hline \multicolumn{3}{c}{ Inducer } \\
\hline $\begin{array}{l}\text { Incidence losses } \\
\text { Contraction losses }\end{array}$ & $\begin{array}{l}\text { Aungier [78] } \\
\text { Impeller }\end{array}$ \\
\hline Blade loading losses & Aungier [78] \\
Aerodynamic loading losses & Aungier [78] \\
Mixing losses & Aungier [78] \\
Friction losses & Schlichting [83] \\
Clearance losses & Aungier [78] \\
\hline & Vaneless Diffuser \\
\hline Friction losses & Aungier [78] \\
\hline & Vaned Diffuser \\
\hline Blockage losses & Aungier [78] \\
Friction losses & Schlichting [83] \\
Incidence losses & Monge [82] \\
Mixing losses & Aungier [78] \\
\hline
\end{tabular}


The methodology aims to integrate different empirical loss models developed for conventional turbomachinery with a real gas equation of state to establish the stage geometry and the performance maps.

Rinaldi et al. [84] performed a numerical study on the centrifugal compressor performance close to the critical point, focusing mainly on the numerical methodology and the interaction between the solver and the real gas model. Monge $[79,82]$ developed a design strategy of a centrifugal compressor for $10 \mathrm{MWe}$ s$\mathrm{CO}_{2}$ power cycle based on Aungier's method, proposing a parameter that ensures the velocity of the flow will not decrease until a limit when the condensation takes place. Also, Zhao et al. [85] conducted a numerical study for the aerodynamic design optimization and the structural analysis of a centrifugal compressor. The same research group investigated how the tip clearance of the centrifugal compressor affects its performance, numerically examining various tip clearance gaps and the pressure losses that they produce [86]. In 2016, Pham et al. [87] established an approach in order to produce the centrifugal compressor map, using a three-dimensional computational fluid dynamics tool. Finally, Liu et al. [88] in 2018 proposed a one-dimensional model that can accurately predict the losses of the impeller, and compared these results with corresponding CFD analysis resulting in a 3\% deviation for the efficiency. In the current work, the approach suggested by Aungier is generally adopted, with several modifications discussed in the following chapters.

\subsubsection{Condensation and two-phase flow investigation}

Condensation is an important issue that is faced particularly in low-pressure steam turbines and is associated with the cavitation problems. However, supercritical carbon dioxide compressors inlet is in the vicinity of the critical point and this problem can also occur at $\mathrm{s}-\mathrm{CO}_{2}$ compression. This is the reason that the inlet conditions of the compressor are commonly shifted away from the critical point because of the possibility of condensation that can occur at the leading edge of the impeller, where the flow accelerates. The acceleration is commonly observed on the suction side of the vane. The acceleration in that region can cause the static conditions to fall into the two-phase region. Even at the compressor's design point, two phase effects are expected in a small region near the leading edge. Moreover, for transient operation, particularly at start-up, condensation is possible at compressor, as usually the initial conditions at start-up are ambient conditions, thus subcritical.

Firstly, Wright et al. [28] at Sandia test facility investigated a centrifugal compressor with s- $\mathrm{CO}_{2}$ operating at two-phase region, reporting that the two-phase effects may not significantly affect the compressor. Monge [79] presented a study on the conditions that could lead to condensation, proposing a limit for velocity acceleration (AMC). Pecnik et al. [89] investigated the condensation for a 
centrifugal compressor's impeller near the $\mathrm{CO}_{2}$ critical point, treating the fluid inside the two-phase flow in equilibrium condition. Baltadjiev [90] presented a study that takes into consideration the metastable region and the droplet generation in non-equilibrium conditions, proposing a criterion for the droplet generation. Also, Ameli et al. [91] investigated the real gas effects near the critical point, examining the accuracy of real gas properties tables created for their numerical models and how the two-phase flow is created at the impeller leading edge.

As a general remark, in specific component studies (i.e. compressor) there exists a tendency to shift the design point to higher inlet temperatures in order to allow for a safety margin towards condensation. However, as will be shown in the next chapter, this may sacrifice cycle's efficiency. Thus, the detailed design of s- $\mathrm{CO}_{2}$ cycles requires a system approach, where the safety margins should be minimized with careful design of sensitive parts (i.e. compressor inlet, low temperature recuperator outlet etc.). These issues are discussed in the pertaining chapters. 


\section{Chapter 3}

\section{Thermodynamic Analysis of Recompression Brayton Cycle}

Many layouts have been investigated for supercritical carbon dioxide applications. The recompression cycle has been theoretically proved to be the most efficient, presenting better efficiencies than Rankine cycles, while the use of a second compressor (recompressor) aims to split the mass flow in the two sides of the low temperature recuperator, eliminating pinch-point problems in heat exchangers. Moreover, the rapid properties' variation of carbon dioxide near the critical point, result in a reduced compression work. The distinct behavior of carbon dioxide demands the use of non-ideal gas approach and the use of specific governing equation of state. The current study performs a thermodynamic analysis of a $600 \mathrm{MW}_{\text {th }}$ cycle, investigating the key parameters of the cycle, such as the compressor's pressure ratio or turbine inlet temperature, and their impact on the system's efficiency. Moreover, an exergy analysis takes place in order to examine which components produce the more significant irreversibilities and require meticulous design.

\subsection{Carbon Dioxide Properties}

Carbon dioxide is a particular fluid, as approaching its critical point it is difficult to distinguish if its behavior is closer to a compressible or an incompressible fluid. The non-ideal state of the fluid gives rise to a set of derived properties that impact the governing equations of compressible flow of real fluids. The parameter employed to clear such behaviors is compressibility factor, which determines how close or far to the ideal gas behavior, is the fluid's behavior.

$$
Z=\frac{P v}{R T}
$$

A value of compressibility factor close to unity translates to an ideal gas behavior, while when compressibility factor presents values down to 0.2 near the critical point the real gas effects are more important. However, the compressibility factor (Z) only gives the magnitude of this deviation, while the gradients of $Z$ with respect to pressure and temperature determine the behavior of the fluid in thermodynamic 
processes. These secondary properties can be related to the isothermal and isobaric compressibilities of a fluid by the following expressions [90]:

$$
\begin{aligned}
& \beta_{T}=-\frac{1}{v}\left(\frac{\partial v}{\partial p}\right)_{T}=\frac{1}{p}-\frac{1}{Z}\left(\frac{\partial Z}{\partial p}\right)_{T} \\
& \beta_{p}=-\frac{1}{v}\left(\frac{\partial v}{\partial T}\right)_{p}=\frac{1}{T}+\frac{1}{Z}\left(\frac{\partial Z}{\partial T}\right)_{P}
\end{aligned}
$$

So, the specific enthalpy and internal energy for real gas are estimated as:

$$
\begin{gathered}
d h=c_{p} d T+v\left(1-\beta_{p} T\right) d p \\
d u=c_{v} d T+p\left(\frac{\beta_{p} T}{\beta_{T} p}-1\right) d v
\end{gathered}
$$

These relationships indicate that whenever $\mathrm{Z}$ is not constant both the enthalpy and the internal energy of a real gas no longer solely dependent on temperature. This has critical implications in the derivation of the isentropic relations for real gas. The expressions for the isentropic exponents $n_{s}$ can be written as

$$
n_{s}=-\frac{v}{p}\left(\frac{\partial p}{\partial v}\right)_{s}=\frac{\gamma}{\beta_{T} p}
$$

So, the speed of sound for a real gas is expressed as follows:

$$
\alpha^{2}=\left(\frac{\partial p}{\partial \rho}\right)_{s}=-v^{2}\left(\frac{\partial p}{\partial v}\right)_{s}=n_{s} p v=n_{s} Z R T
$$

Carbon dioxide varies its compressibility factor and all the other thermodynamic properties really abruptly approaching the critical point as it is presented in Figure 3.1 and Figure 3.2. It can be observed that the compressibility factor (Figure 3.2) for the critical conditions presents a value around 0.2 , which quickly varies when the pressure and the temperature increase. Especially for pressures above $20 \mathrm{MPa}$ the compressibility factor takes values around 0.5 which remain almost constant as the temperature increases. Moreover, properties such as the specific heat capacity, density and sound speed present the same behavior at the pseudocritical region and seem to remain almost constant as the conditions get away from this region. 

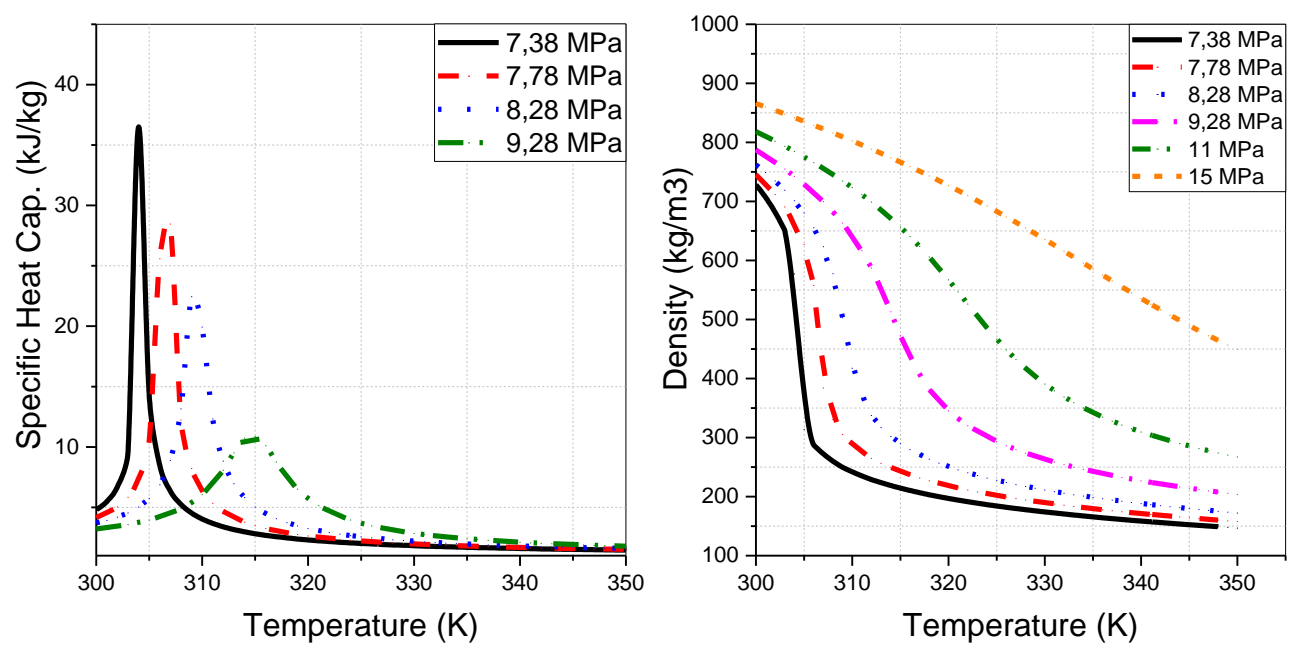

Figure 3.1. Specific heat-temperature and density-temperature diagrams of $\mathrm{CO}_{2}$ for various pressures.
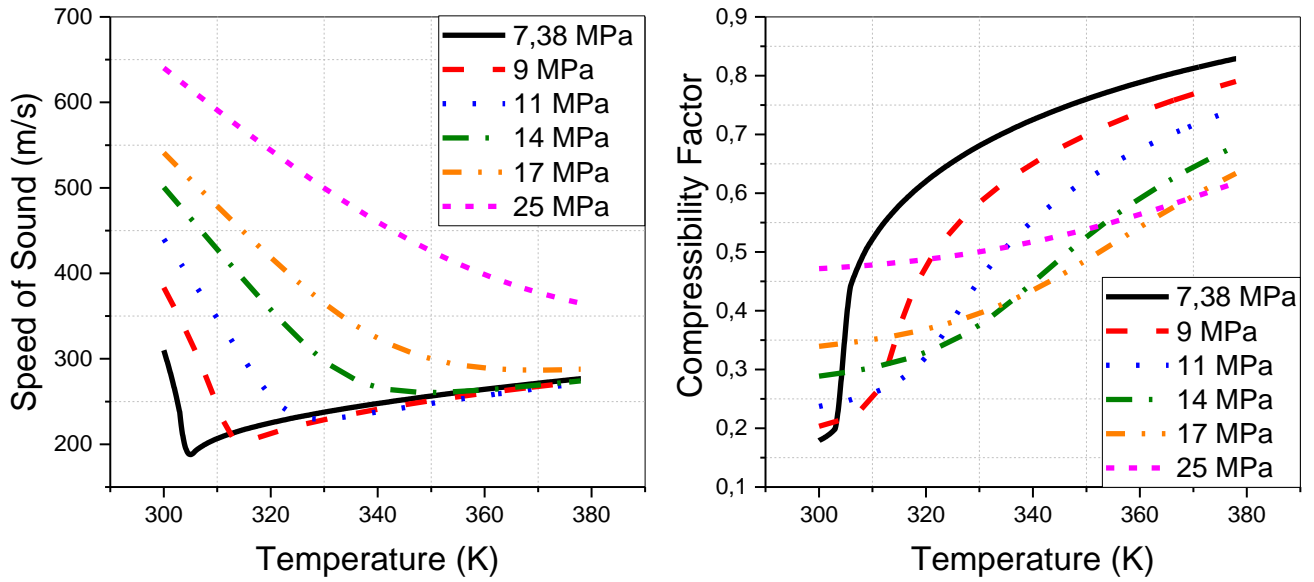

Figure 3.2. Carbon dioxide speed of sound and compressibility factor variation with temperature for different pressures.

A variety of equation of state (EOS) models have been proposed, presenting different accuracy depending on the range of thermodynamic states of interest. A study by Lüdtke [92] compared various common real gas models, proving that the Lee and Kesler model (1975) modified by Ploker, Knapp, and Prausnitz (1978) is the best, presenting a deviation with experimental data less than $2 \%$.

However, the model of Span and Wagner (SW) [93] was published more recently (1996) and developed particularly for carbon dioxide, is more widespread in the carbon-dioxide properties modeling in science. Span-Wagner equation of state (EOS) is a real gas model especially for $\mathrm{CO}_{2}$, which is expressed in terms of 
Helmholtz energy and covers the range from the triple point up to $1100 \mathrm{~K}$ and 80 MPa for temperature and pressure respectively. Lately, Baltadjiev [90] compared the above mentioned EOS models with special treatment in the region of the critical point, indicating that the models are in good agreement in the operational range of a compressor for supercritical $\mathrm{CO}_{2}$ applications, but for simulation closer to the critical point $\mathrm{SW}$ model is more recommended.

The SW EOS in terms of Helmholtz energy is expressed as:

$$
\begin{gathered}
\frac{A(\rho, T)}{(R, T)}=\varphi(\delta, \tau)=\varphi^{o}(\delta, \tau)+\varphi^{r}(\delta, \tau) \\
\varphi^{r}(\delta, \tau)=\sum_{i=1}^{7} n_{i, j} \delta^{d_{i}} \tau^{t_{i}} \\
+\sum_{i=8}^{34} n_{i} \delta^{d_{i}} \tau^{t_{i}} e^{-\delta^{c_{i}}} \\
+\sum_{35}^{39} n_{i} \delta^{d_{i}} \tau^{t_{i}} e^{-a_{i}\left(\delta-\varepsilon_{i}\right)^{2}-\beta_{i}\left(\tau-\gamma_{i}\right)^{2}} \\
+\sum_{i=40}^{42} n_{i} \Delta^{b_{i}} \delta e^{-\left[c_{i}(\delta-1)^{2}-D_{i}(\tau-1)^{2}\right]} \\
\varphi^{o}(\delta, \tau)=\ln (\delta)+\alpha_{1}{ }^{o}+\alpha_{2}{ }^{o} \tau \\
\quad+\alpha_{3}{ }^{o} \ln (\tau)+\sum_{i=4}^{8} \alpha_{i}^{o} \ln \left[1-\exp \left(-\tau \vartheta_{i}^{o}\right)\right]
\end{gathered}
$$

Where, $=\rho / \rho_{c}, \tau=T / T_{c}$ are the reduce parameters for density and temperature and

$$
\Delta=\left\{(1-\tau)+A_{i}\left[(\delta-1)^{2}\right]^{1 /(2 \beta)_{i}}\right\}^{2}+B_{i}\left[(\delta-1)^{2}\right]^{\alpha_{i}}
$$

$\varphi$ is the dimensionless Helmholtz function, while $\varphi^{o}$ is the ideal gas part of Helmholtz function and $\varphi^{r}$ is the residual part of Helmholtz function. The last terms $(i=40-42)$ represent the effect of the critical point, with $\Delta$ being an exponential function that damps their influence outside the critical region. All the coefficients can be found in [93]. The Span and Wagner model is used by NIST Refprop for thermodynamic properties estimation of $\mathrm{CO}_{2}$.

Table 3.1 presents the deviation of density between the model proposed by Span-Wagner and other models for carbon dioxide, for conditions in the pseudocritical region. It is observed that the ideal gas approach presents the highest 
deviation from SW model, but none of the other equations of state deviates less than $13 \%$.

Table 3.1. Deviation of density between Span-Wagner model and other equations of state.

\begin{tabular}{lcc}
\hline EOS & $\begin{array}{c}\text { Density }\left(\mathrm{kg} / \mathrm{m}^{3}\right) \\
\text { at } 7.69 \mathrm{MPa}, 304.3 \mathrm{~K}\end{array}$ & Deviation (\%) \\
\hline Span-Wagner & 598.81 & - \\
Perfect gas & 133.41 & -77.7 \\
Redlich-Kwong & 484.55 & -19.1 \\
Aungier/Redlich-Kwong & 519.14 & -13.3 \\
Soave/Redlich-Kwong & 477.8 & -20.2 \\
\hline
\end{tabular}

Moreover, Figure 3.3 presents the specific heat variation with temperature for a constant pressure of 7.69 MPa for Span-Wagner and Peng-Robinson models. It is obvious that Peng-Robinson cannot predict the rapid change around the critical point, while the SW model seems to approach it in a more efficient way. However, when the temperature increases in the range that $\mathrm{CO}_{2}$ approaches perfect gas behavior, the two models present good agreement. Therefore, for the cycle components that operate close to critical point, SW model has to be used. On the other hand, for a heat exchanger that operates in the temperature range far from critical point, Peng-Robinson model could also be used.

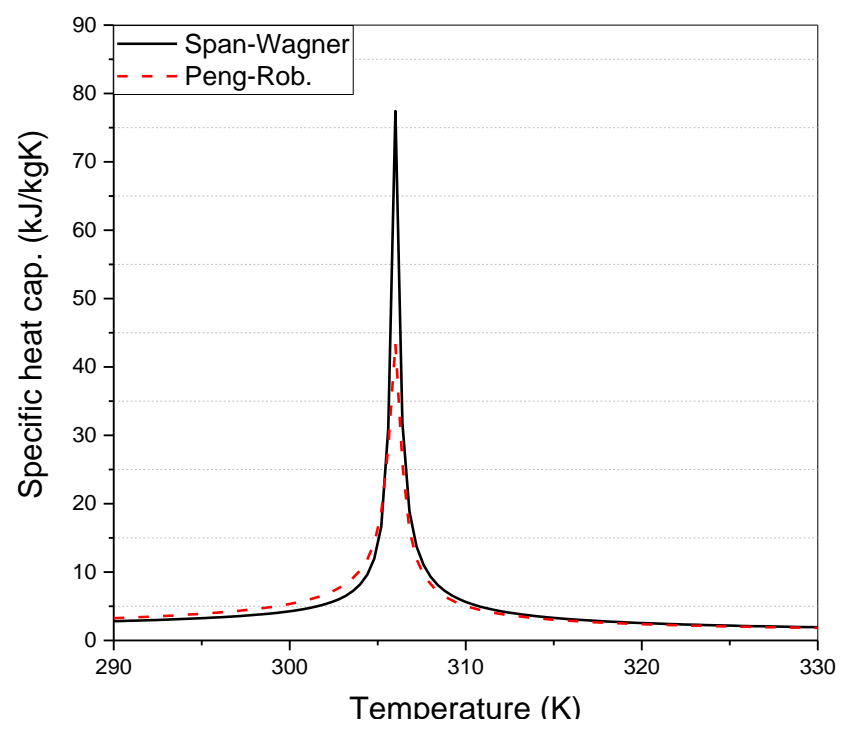

Figure 3.3. Specific heat capacity variation for Span-Wagner and Peng-Robinson equations of state. 


\subsection{Recompression Cycle Layout}

The recompression s- $\mathrm{CO}_{2}$ Brayton cycle layout improves efficiency by introducing a second compressor (a recompressing compressor), which compresses a part of the working fluid before it enters the pre-cooler (Figure 3.4). The split of the flow aids to overcome the pinch-point problem, mainly for the low temperature recuperator. A short description of the recompression Brayton cycle follows:

The main compressor (states 1-2) compresses the largest fraction of the flow to a high pressure and temperature. Subsequently, the flow is preheated through the low temperature recuperator (8-3a) to an outlet temperature level that matches that of the recompressing compressor outlet (states 8-3b), and the two partial flows are merged (state 3 ). Then, the entire fluid flow is further preheated in the high temperature recuperator (states 3-4), until it reaches the inlet temperature of the primary heat exchanger where the heat addition takes place (states 4-5). The fluid exits the primary heat exchanger at the highest cycle temperature which is about $550^{\circ} \mathrm{C}$. At this temperature, it enters the turbine, where the fluid is expanded (states 5-6) to produce mechanical power. After leaving the turbine, the fluid is cooled in the HTR (states 6-7) and LTR (states 7-8), where high temperature heat from the working fluid is transferred to preheat the cooler high pressure flow.

After leaving the LTR the flow is split (state 8). One part is recompressed to high pressure (states 8b-3) and the other is cooled in the pre-cooler to the main compressor inlet temperature (states 8a-1).

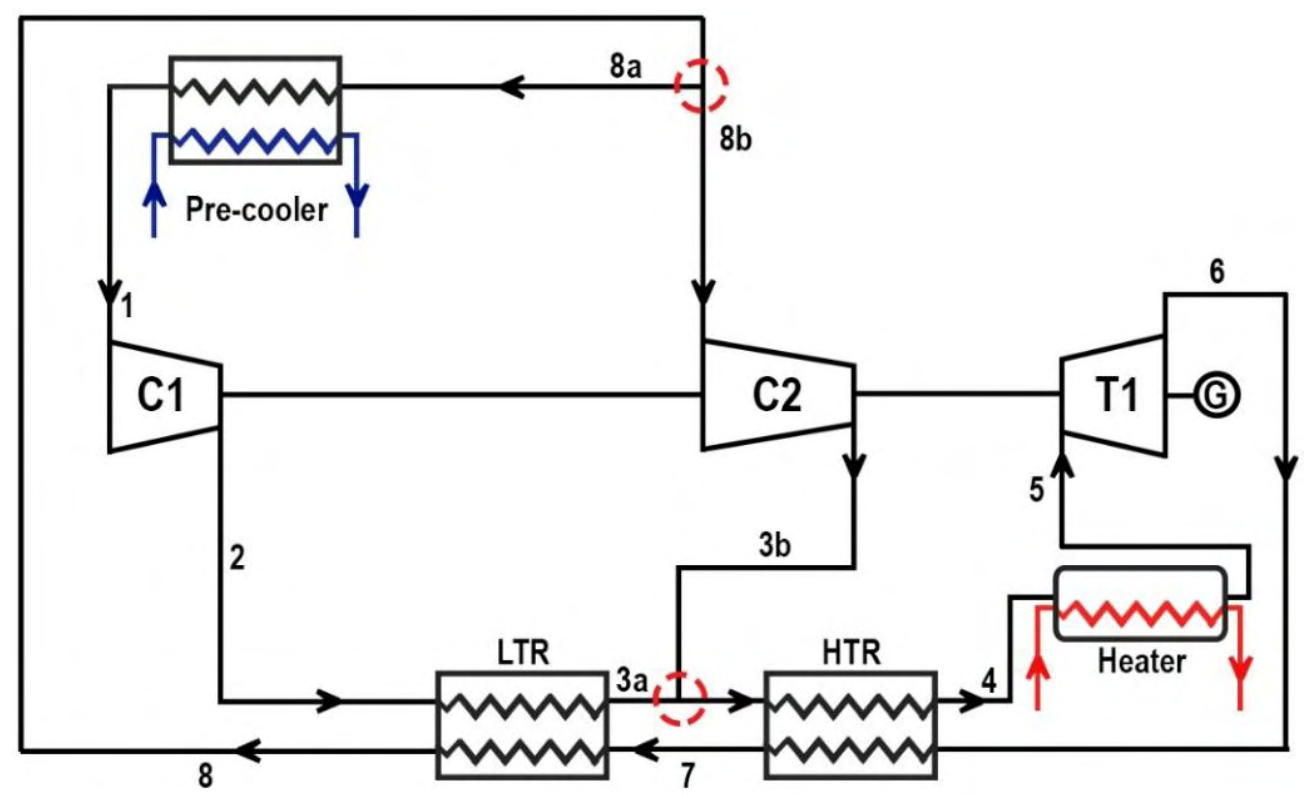

Figure 3.4. Recompression s- $\mathrm{CO}_{2}$ Brayton cycle layout. 
Figure 3.5 and Figure 3.6 present the cycle in temperature-entropy and pressureenthalpy diagrams. It can be observed from these two diagrams that the compressor inlet is just above the saturation line of carbon dioxide. This is the reason that the main compressor of the system presents great challenge for its design, in order to avoid possible condensation during the compression process.

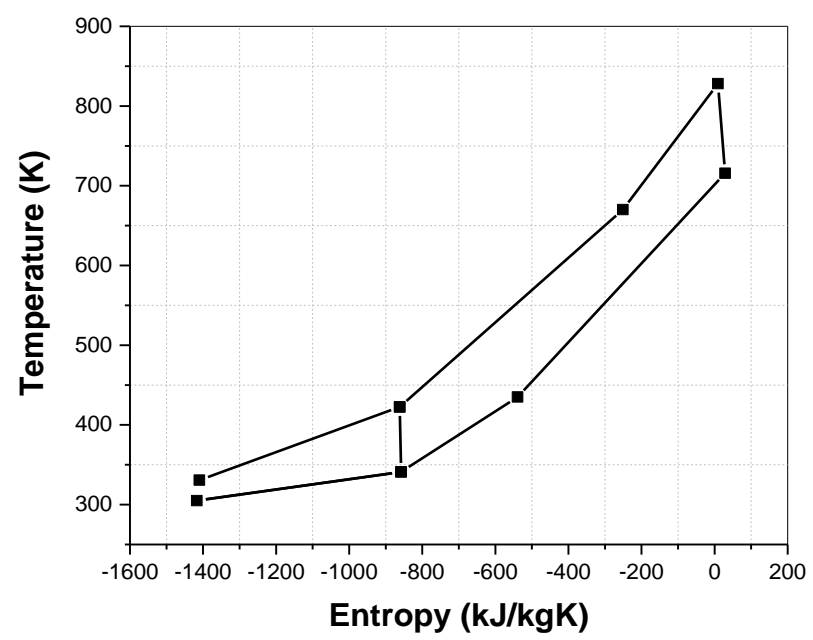

Figure 3.5. Temperature-entropy diagram for recompression s- $\mathrm{CO}_{2}$ Brayton cycle.

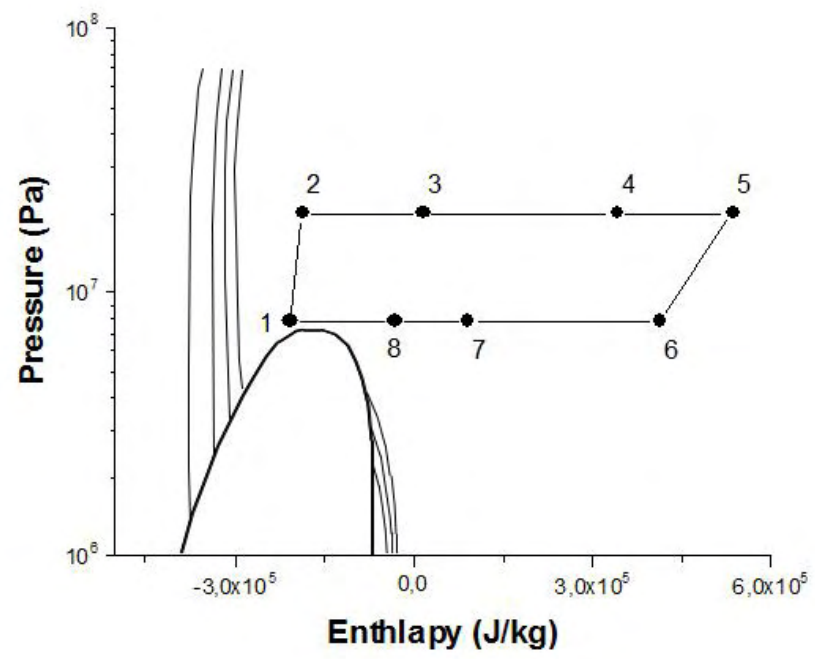

Figure 3.6. $\mathrm{LogP}$-enthalpy diagram for recompression s- $\mathrm{CO}_{2}$ Brayton cycle, presented with the saturation line.

The modeling tool employed in this thermodynamic study was Engineering Equation Solver (EES), which includes libraries with thermodynamic properties 
for the majority of the possible working mediums, and offers to the user the possibility to develop the appropriate thermodynamic and fluid mechanics equations and equilibriums describing the main system components (compressors, turbine and heat exchangers). EES gives the opportunity to approach carbon dioxide as ideal or real gas, employing the Span-Wagner equation of state for the real gas approach, such as the thermophysical data derived by NIST Refprop. The main input parameters for the simulated Brayton cycle are included in the Table 3.2 .

Table 3.2. Input design parameters for the recompression cycle.

\begin{tabular}{cc}
\hline Parameter & Design Point Value \\
\hline Heater input (MW) & 600 \\
Total mass flow (kg/s) & 3180 \\
High cycle pressure (MPa) & 20 \\
High cycle temperature (K) & 828 \\
Compressor efficiency & 0.85 \\
Recompressor efficiency & 0.85 \\
Turbine efficiency & 0.9 \\
\hline
\end{tabular}

The results of the steady-state EES modeling are presented in tabular form in Table 3.3. EES provides entropy and enthalpy at a reference state of $298 \mathrm{~K}$ and 1 atm [94]. The pressure drop in the heat exchangers was neglected for the primary thermodynamic analysis.

Table 3.3. Results for each operating point of the recompression cycle.

\begin{tabular}{cccccc}
\hline No & $\begin{array}{c}\text { Pressure } \\
(\mathbf{M P a})\end{array}$ & $\begin{array}{c}\text { Temperature } \\
(\mathbf{K})\end{array}$ & $\begin{array}{c}\text { Enthalpy } \\
(\mathbf{k J} / \mathbf{k g})\end{array}$ & $\begin{array}{c}\text { Entropy } \\
(\mathbf{k J} / \mathbf{k g K})\end{array}$ & $\begin{array}{c}\text { Specific volume } \\
(\mathbf{m} \mathbf{3} / \mathbf{k g})\end{array}$ \\
\hline 1 & 20 & 330.8 & 336.78 & 1.3829 & 0.0014437 \\
2 & 20 & 422.6 & 522.29 & 1.8775 & 0.003047 \\
3 & 20 & 670.03 & 846.72 & 2.4879 & 0.00636 \\
4 & 20 & 828.1 & 1041.3 & 2.7485 & 0.00809 \\
5 & 7.7 & 715.7 & 917.23 & 2.7682 & 0.01734 \\
6 & 7.7 & 434.95 & 596.69 & 2.2002 & 0.009473 \\
7 & 7.7 & 341.1 & 474.86 & 1.8825 & 0.00585 \\
8 & 7.7 & 305 & 300.27 & 1.3262 & 0.00157 \\
\hline
\end{tabular}


In respect of the optimal sizing and placement of the cycle in the thermodynamic diagrams of carbon dioxide (Figure 3.6), the effect of various parameters of the cycle on cycle performance was investigated.

\subsubsection{Compressor suction temperature and pressure ratio effect}

This section presents how the compressor outlet temperature and pressure affect the system performance. Even if turbine inlet temperature has more significant impact on cycle's efficiency, the compressor pressure ratio can be crucial for an efficient cycle. Moreover, the compressor suction temperature is connected with the outlet flow temperature of the low temperature recuperator, affecting both the recuperators' effectiveness and the cycle efficiency, since the specific heat capacity's variation is still decisive in this temperature and pressure range.

\section{a. Effect of pressure ratio}

A sensitivity analysis for the compressor pressure ratio and the corresponding efficiency was carried out for a fixed geometry of the recuperators, with the results presented in Figure 3.7. For a supercritical $\mathrm{CO}_{2}$ cycle the thermal efficiency increases at first presenting a maximum efficiency of $46 \%$ at an optimal pressure 2.55 , and then decreases because of the recuperators' disability to increase the cycle efficiency at high discharge pressures.

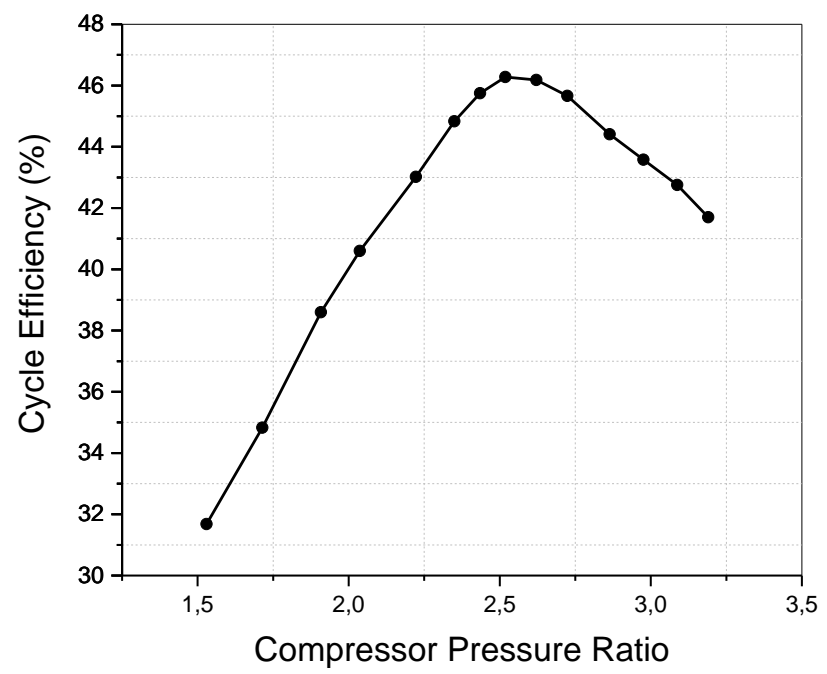

Figure 3.7. Thermal efficiency variation as compressor pressure ratio increases. 


\section{b. Effect of minimum operating temperature}

Moreover, the compressor inlet temperature has a significant impact on the cycle efficiency. Low inlet temperatures, closer to critical conditions, are associated with abrupt property variations. Thus, a change in compressor inlet temperature could lead to a significant change in the efficiency of the compression process, but could also play an important role in the recuperators' effectiveness variation.

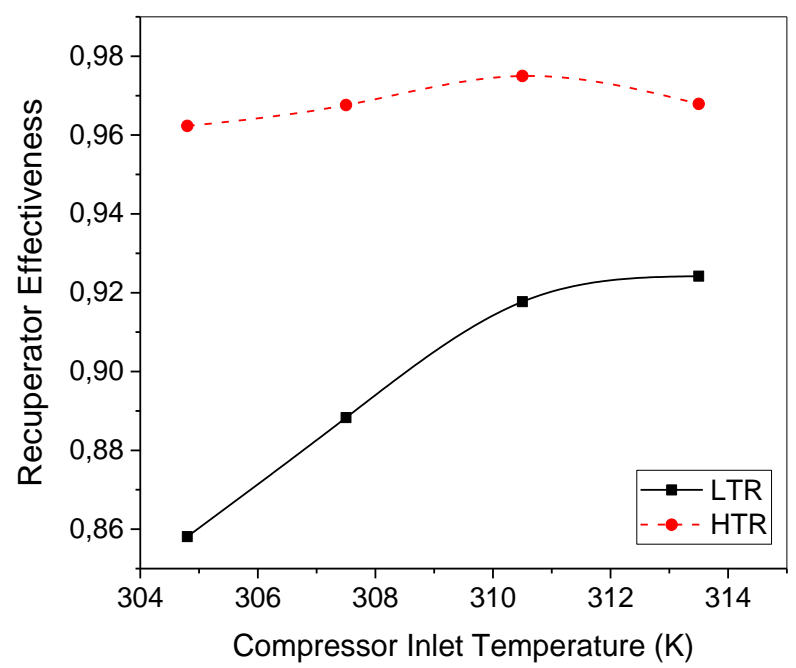

Figure 3.8. Recuperators' effectiveness as function of compressor inlet temperature.

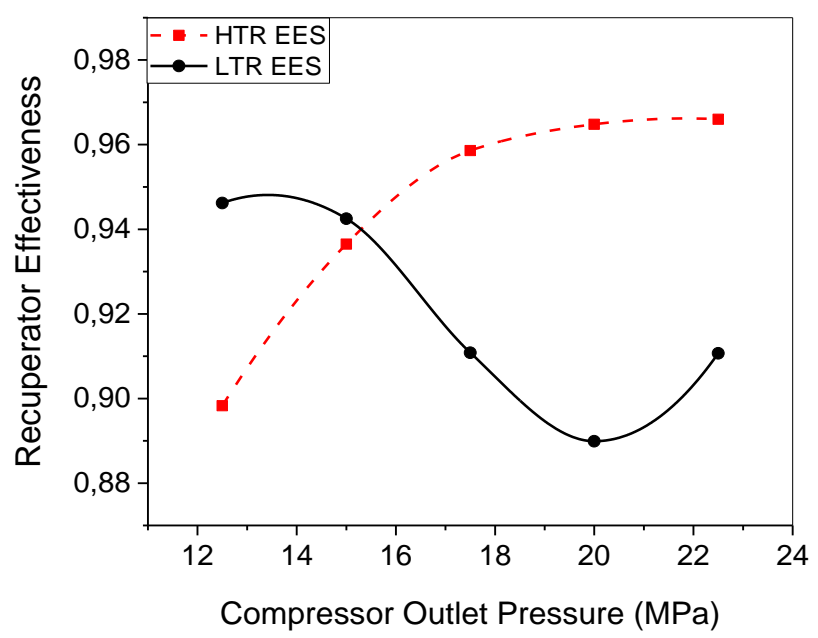

Figure 3.9. High \& low temperature recuperator effectiveness as function of compressor discharge pressure.

As the main compressor inlet temperature increases, the effectiveness of both HT and LT recuperators change. This is affected by the operation of the carbon 
dioxide far from the critical point. The effectiveness of the high temperature recuperator remains high and slightly increases to a maximum. The low temperature recuperator effectiveness remains at a lower level and is significantly improved by increasing compressor inlet temperature in the specific range of Figure 3.8. The level of the estimated effectiveness seems to be achievable according to published measurement results [62].

It is evident that the compression process has a significant impact to recuperator effectiveness and eventually to cycle performance. Thus, it is important to investigate the maximum cycle operating conditions and the way they affect cycle performance. Figure 3.9 presents the effectiveness of the high and low temperature recuperators. It can be observed that the compressor outlet pressure significantly affects the effectiveness, especially of the HTR. It seems that no further gains are possible above $20 \mathrm{MPa}$. On the other hand, for the LTR a decreasing effectiveness is predicted until the pressure reaches $20 \mathrm{MPa}$, while above this pressure the effectiveness improves. This change occurs because of the change of the recompressed mass flow that decreases after $20 \mathrm{MPa}$, affecting the temperatures of the hot heat exchanger's side.

Figure 3.10 show the cycle's efficiency improvement for increasing high pressure of the cycle and increasing the turbine entry temperature (TET), showing the effect of the combination of compressor outlet pressure and TET on the cycle efficiency. It can be observed that the temperature improves the efficiency almost linearly, although the compressor outlet pressure is not really beneficial for the cycle's efficiency.



Figure 3.10. Efficiency improvement as turbine entry temperature increases for various cycle's maximum pressures.

From the above analysis, it is obvious that the cycle performance is strongly affected by operating conditions. The final load of each system's component as 
derived by the used modeling tool and the same $600 \mathrm{MW}_{\text {th }}$ examined by Dostal, is presented in Table 3.4 .

Table 3.4. Operating characteristics of the power system for the design parameters

\begin{tabular}{cccc}
\hline Parameter & $\begin{array}{c}\text { Current } \\
\text { model }\end{array}$ & $\begin{array}{c}\text { Reference } \\
\text { model [3] }\end{array}$ & $\begin{array}{c}\text { Difference Ref.- } \\
\text { current model (\%) }\end{array}$ \\
\hline$\dot{\mathbf{Q}}_{\text {LTR }}(\mathbf{M W})$ & 385.5 & 398.8 & 3.3 \\
\hline$\dot{\mathbf{Q}}_{\text {HTR }}(\mathbf{M W})$ & 1011 & 985.51 & 2.5 \\
\hline$\dot{\mathbf{Q}}_{\text {Heater }}(\mathbf{M W})$ & 603.6 & 600 & 0.6 \\
\hline$\dot{\mathbf{Q}}_{\text {Prec }}(\mathbf{M W})$ & 332.1 & 328.4 & 1.1 \\
\hline$\dot{\mathbf{W}}_{\text {comp }}(\mathbf{M W})$ & 107 & 112 & 4 \\
\hline$\dot{\mathbf{W}}_{\text {turb }}(\mathbf{M W})$ & 391.1 & 383.7 & 1.8 \\
\hline $\boldsymbol{\eta}_{\text {cycle }}$ & 0.462 & 0.4527 & 2 \\
\hline
\end{tabular}

It can be observed that the heater's and recuperators' load is the largest of the components, since the recompression cycle allows to recuperate large thermal energy quantities during the recuperation process. Moreover, the power of the compression process constitutes only $27 \%$ of the turbine-produced power, leading to an increased cycle's net power, which consists a main advantage of the s- $\mathrm{CO}_{2}$ Brayton cycles.

\subsubsection{Exergy analysis}

A second thermodynamic law analysis took place in order to examine the thermodynamic losses that cause exergy destruction in a power cycle. The exergy of an energy system is defined as the maximum work that can be produced by the interaction between a system and a specified reference environment. An exergy analysis is performed in order to reveal the component that produces the most significant irreversibilities, thus the component that can be improved more. The general exergy balance for a system is defined as:

$$
\text { Exergy_inlet-Exergy_outlet-Exergy_consumed }=\text { Exergy_accumulated }
$$

For a steady-state state system, no accumulation is considered in the system. The exergy that enters the system is estimated as:

$$
\dot{E}_{i}=\left(1-\frac{T_{o}}{T_{H}}\right) \dot{Q}_{i}
$$


Where $T_{o}$ corresponds to the environmental reference temperature, $\dot{Q}_{\iota}$ and $T_{H}$ to the heat addition and the average temperature (between inlet and outlet) of the process respectively. The exergy output of the system corresponds to the net work output. The exergy consumption corresponds to the exergy losses produced by the irreversibilities of each component, the destruction of exergy in the heat transfer processes and the exergy rejected by the cooler.

The exergy change of an open steady-state flow is defined as flow exergy and is computed by the following equation (3-13), while Figure 3.11 presents the temperature-flow exergy diagram for the recompression cycle.

$$
\dot{E}_{i}=\dot{m}\left[\left(h_{i}-h_{o}\right)-T\left(s_{i}-s_{o}\right)\right]
$$

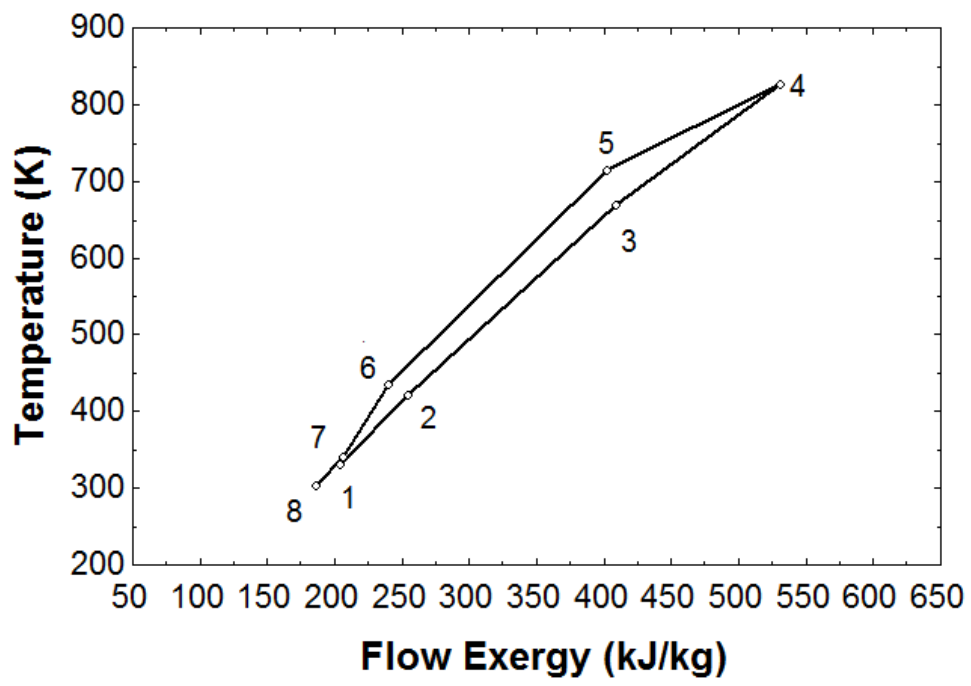

Figure 3.11. Temperature-exergy diagram of the cycle.

The exergy loss of a fluid for a process is expressed as:

$$
\dot{E}_{\text {loss }}=\dot{m} T_{o} \Delta s_{G}
$$

where $\dot{m}$ is the mass flow rate and $\Delta s_{G}$ the entropy generated through the process. So in order to obtain the exergy loss for the compressor, the turbine and a heat exchanger, the following expressions are applied:

$$
\begin{gathered}
\dot{E}_{\text {loss }, \text { comp }}=\dot{m}_{c} T_{o}\left(s_{2}-s_{1}\right) \\
\dot{E}_{\text {loss }, \text { turb }}=\dot{m}_{t} T_{o}\left(s_{5}-s_{4}\right) \\
\dot{E}_{\text {loss }, H E X}=\dot{m}_{\text {in }}\left[\left(h_{\text {out }}-h_{\text {in }}\right)-T_{o}\left(s_{\text {out }}-s_{\text {in }}\right)\right]
\end{gathered}
$$


The exergy loss was estimated for every component of the recompression cycle and the results are presented in the following Figure 3.12 and Table 3.5. The mass flow used in the equations must be adjusted depending on the component, while the low temperature recuperator deserves further attention, since there are two streams with different mass flow rates running the heat exchanger.

Table 3.5. Exergy losses for each component of the recompression s- $\mathrm{CO}_{2}$ Brayton cycle.

\begin{tabular}{lc}
\hline Component & Exergy loss (MW) \\
\hline HT Recuperator & 25 \\
LT Recuperator & 9.2 \\
Pre-cooler & 22.8 \\
Main Compressor & 2.3 \\
Recompressor & 0.5 \\
Turbine & 17.2 \\
\hline
\end{tabular}

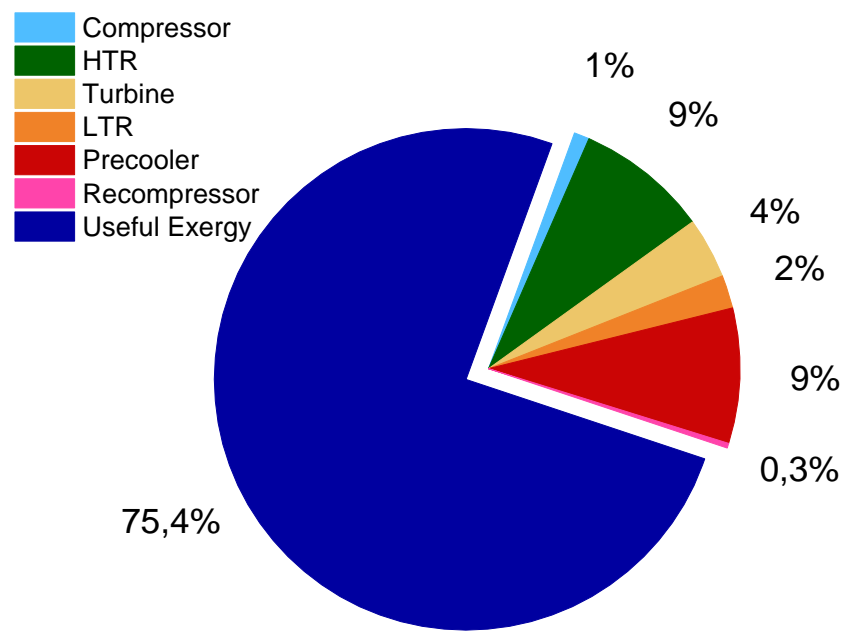

Figure 3.12. Exergy balance of the recompression $\mathrm{s}-\mathrm{CO}_{2}$ Brayton cycle.

The exergy efficiency of the recompression cycle can be defined by the ratio of net exergy output (which corresponds to the net work output as referred above) to the exergy input through the heat source, which is expressed by the following relationship: 


$$
\eta_{I I}=\frac{\dot{W}_{T}-\dot{W}_{m c}-\dot{W}_{\text {recomp }}}{\dot{E}_{i n}}
$$

Finally, Figure 3.13 presents how cycle exergy efficiency varies as turbine entry temperature increases from $530^{\circ} \mathrm{C}$ to $630^{\circ} \mathrm{C}$, showing at the same time the variation of cycle thermal efficiency. It is observed that second law efficiency follows the same graphical behavior with the first law efficiency in the referred operating range, thus after a maximum value it starts decreasing in opposition with thermal efficiency. In the restricted temperature range that was examined, the efficiencies present linear variation. However, the dependence of efficiencies for higher temperatures was not investigated, as the proposed applications could not overcome this temperature range.

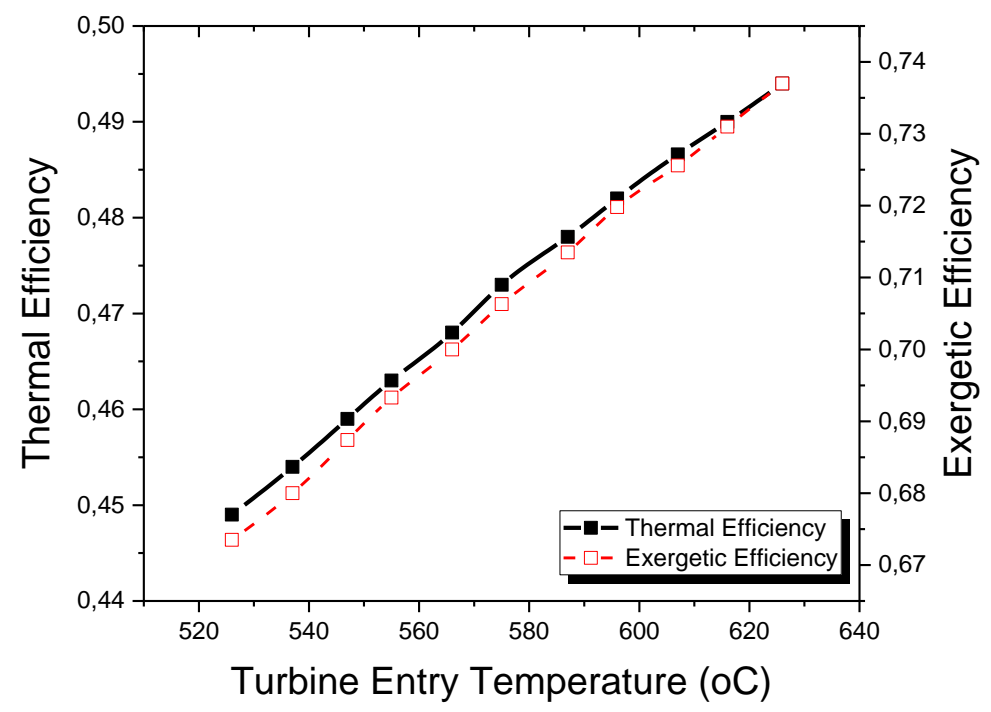

Figure 3.13. Thermal and exergy efficiency of the cycle as the turbine entry temperature increases.

\subsection{Conclusions}

This chapter presents a thermodynamic analysis of a supercritical carbon dioxide Brayton cycle with recompression for power generation. The carbon dioxide properties close to the pseudo-critical region were discussed and the SpanWagner model that is considered the most accurate for carbon dioxide was presented. Moreover, the recompression cycle layout and the design parameters of 
a 600 MWth cycle were presented. The main parameters that affect the components and the cycle efficiency, such as compressor inlet temperature, compressor pressure ratio and turbine inlet temperature, were investigated, leading in an optimum pressure ratio of 2.55. Finally, an exergy analysis was conducted, signifying that the pre-cooler and the high temperature recuperator present the maximum exergy destruction. For the cycle's design point, the thermal efficiency was proved around $46 \%$ and the exergetic efficiency $70 \%$. The components that destroy the maximum amount of exergy are proved to be the high temperature recuperator and the pre-cooler. 


\section{Chapter 4}

\section{Design and Modeling Methods of Printed- Circuit Heat Exchangers}

The thermodynamic analysis showed that the cycle heat exchangers need thorough investigation, since a large amount of irreversibilities is produced there. This chapter presents the methods used in order to model and analyze the printedcircuit heat exchangers thermal-hydraulic performance. The heat exchangers design requirements are presented and the governing equations are discussed.

The PCHEs investigation included one-dimensional steady-state modeling, presenting a segmental analysis, in order to define the heat transfer coefficient, the effectiveness and the pressure drop of the heat exchangers. Moreover, the heat exchangers dynamic performance is investigated under various transient cases. Furthermore, a three-dimensional geometry of a PCHE is investigated with computational fluid dynamics in order to examine the heat transfer phenomena and finally an experimental study of a PCHE is presented. The results are compared in order to validate the models and useful conclusions are drawn

\subsection{Selection of Heat Exchangers Design}

Before starting the design, the requirements of the proposed heat exchanger duty are screened to check for feasibility. This initial screening should review if:

- The materials of the heat exchanger can withstand the cycle's design pressure and temperature.

- The working supercritical carbon dioxide is compatible with the heat exchanger type and free from effects that can cause fouling or other excessive blockage.

- There exists an energy balance for the specified flow-rates and temperatures.

- The presented pressure drop that makes the application affordable.

- The required thermal effectiveness can be accomplished by a reasonable heat transfer area.

The first above design choices (especially the first) influence the material choice and can influence the designer to select one heat exchanger type in preference to another. For example, a specification of a higher design temperature requiring a 
higher alloy of greater cost may result in selection of a FPHE solution, as FPHE construction tends to require less raw material. Specification of a higher design pressure results in selection of a PCHE solution, which due to fin forming limitations can be designed to satisfy a higher design pressure than a FPHE design.

Since the recompression Brayton s- $\mathrm{CO}_{2}$ cycles operates with high pressures (up to $20 \mathrm{MPa}$ ), while the temperatures present a range that is common for waste heat recovery applications, the heat exchanger selection will be the printed-circuit heat exchanger. In the following sections of Chapter 4 the thermal-hydraulic performance of the heat exchanger is discussed.

\subsection{Steady-State One-Dimensional Modeling}

For the development of an analytical performance estimation method for heat exchangers design, the most common calculation methods are the mean logarithmic temperature difference method (LMTD) and the effectiveness number of transfer units ( $\varepsilon$-NTU). However, any of these methods are not suitable for printed-circuit heat exchangers with s- $\mathrm{CO}_{2}$, as they are based on the assumption that the heat transfer fluids have constant specific heat and cannot be applied in the case of supercritical carbon dioxide that its properties vary significantly near the critical point. As a result, the length of the heat exchanger is discretized into a number of elements in series, where the heat is transferred between the hot and cold streams in each element. The heat transfer is considered that takes place in one dimension (x-direction) as presented in Figure 4.1, and consists the most common approach of PCHE study, in order to calculate the effectiveness and other pertaining parameters of every element $[54,60,62,95]$.

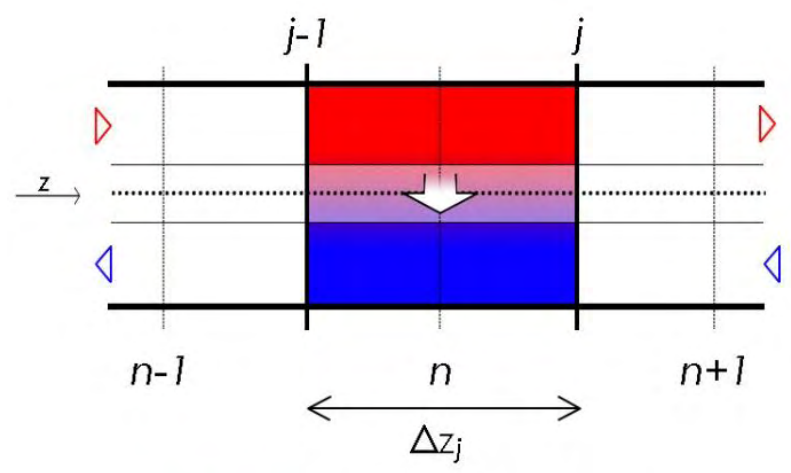

The $n$-th control volume

Figure 4.1. PCHE cross-flow discretization stencil [96].

There are two methods to define the heat exchanger size. The first method need to specify a fixed effectiveness and calculate the necessary conductance (UA) for 
each node, resulting in a total UA value for the entire heat exchanger and calculating the corresponding effectiveness, and the second method requires just the specification of two inlet temperatures and one outlet temperature. For this problem, the second approach was adopted. The following sections present the procedure for the heat transfer coefficient and the pressure drop for a printed-circuit heat exchanger. This procedure describes the steady-state modeling of the PCHE.

\subsubsection{Overall heat transfer coefficient}

The heat transferred between the hot and cold streams is estimated for each element with the following Equations (4-1)-(4-3):

$$
\begin{gathered}
\dot{Q}_{i}=\dot{m}_{c}\left(h_{i, \text { out }}-h_{i, \text { in }}\right)_{c}=\dot{m}_{h}\left(h_{i, \text { out }}-h_{i, \text { in }}\right)_{h} \\
\dot{Q}=\frac{\Delta T}{R_{\text {tot }}} \\
R_{\text {tot }}=\frac{1}{U A}=\frac{1}{a_{c} A}+\frac{\ln \frac{d_{o}}{d_{i}}}{2 \pi k L}+\frac{1}{a_{h} A}
\end{gathered}
$$

For the estimation of the heat transfer resistance, the geometrical dimensions of the PCHE have to be used, as they are presented in the Figure 4.2.
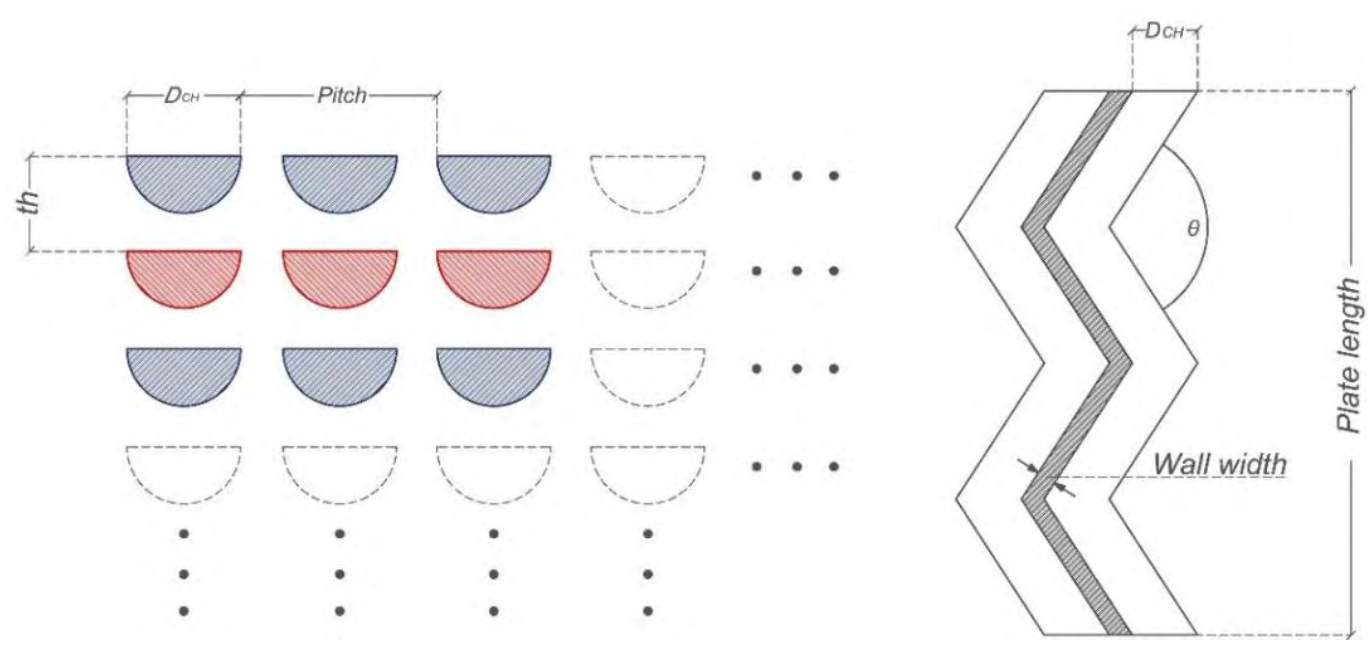

Figure 4.2. Geometrical characteristics of PCHE channels.

Finally, the heat transfer coefficient is given by the equation: 


$$
a=\frac{N u}{d_{h}} k
$$

where the hydraulic diameter is expressed as:

$$
d_{h}=\frac{\pi d_{c}^{2}}{2\left(\pi \frac{d_{c}}{2}+d_{c}\right)}
$$

The thermal conductivity of the fluid $k$ can be estimated as a function of pressure and temperature of the carbon dioxide. The Nusselt number has to be defined in order to estimate the heat transfer coefficient. However, the heat transfer coefficient may not be accurately predicted from theory since the flow passages through PCHE are too complex. The correlations for designing commercial PCHEs are not available in the open literature. However, extensive experimental and numerical studies have been conducted and thermal-hydraulic models for designing PCHEs with different types of channel geometry have been developed. In the following sections various models for the Nusselt number and friction factor are presented in order to obtain the heat transfer coefficient.

The straight channel consists a channel type of a conventional plate type heat exchanger. The Nusselt number was evaluated using the Gnielinski [97] equation:

$$
N u=\frac{\frac{f}{8} \cdot(\operatorname{Re}-100) \cdot \operatorname{Pr}}{1+12.7 \cdot \sqrt{\frac{f}{8}} \cdot\left(\operatorname{Pr}^{2 / 3}-1\right)}
$$

where Pr is the Prandtl number and $\mathrm{f}$ the friction factor determined by:

$$
f=\left(\frac{1}{1.8 \log \mathrm{Re}-1.5}\right)^{2}
$$

The Gnielinski correlation is valid for Reynolds numbers between 2300 and $5 \times 10^{6}$ and Prandtl numbers between 0.5 and 2000. For laminar flows, Hesselgraves [98] recommends a Nusselt number of 4.089. Dostal [3] proposes an interpolation to calculate the Nusselt in the transition region $(2300 \leq \operatorname{Re} \leq 5000)$.

$$
N u=4.089+\frac{N u_{\mathrm{Re}=5000}-4.089}{2300}(\mathrm{Re}-2300)
$$

where Reynolds number is estimated by the equation:

$$
R e=\frac{\rho v d_{h}}{\mu}
$$


The Gnielinski equation has been tested in different heat exchangers and conditions. Kruizenga et al. [40] studied the heat transfer coefficient in a straight channel PCHE with $\mathrm{CO}_{2}$ under supercritical pressure. They compare the results with several correlations and conclude that the Gnielinski equation gives results in good agreement with experimental data, within a difference of $20 \%$ at conditions far from the pseudo-critical temperature. Moreover, it is reported that Nusselt numbers mostly overpredicted by values with an error close to $60 \%$ when the $\mathrm{CO}_{2}$ is close to the critical region.

On the other hand, the wavy or zigzag channel increases the heat transfer with moderate friction loss. Extensive research has been carried out on wavy-channel PCHEs, as it was presented in Chapter 2. For this type of channels, the Nusselt number and the friction factor is calculated using new correlations. Baik et al [62] proposed a new correlation:

$$
\begin{gathered}
N u=0.8405 R e^{0.5704} \operatorname{Pr}^{1.08} \\
f=0.0748 R e^{-0.19}
\end{gathered}
$$

While another correlation is proposed by Kim for applications with $2000<$ $\operatorname{Re}<58000$ and $0.7<\operatorname{Pr}<1.0[60]$ :

$$
\begin{gathered}
N u=0.0292 R e^{0.8137 \pm 0.0050} \\
f=(0.2515 \pm 0.0097) R e^{-0.2031 \pm 0.0041}
\end{gathered}
$$

\subsubsection{Effectiveness calculation}

Since the heat transferred between the hot and cold streams has been defined, an iterative method for the estimation of enthapy outlet of each stream can be applied.

$$
\begin{gathered}
h_{h, i}=h_{h, i-1}+\frac{\dot{Q}}{N \cdot \dot{m}_{h}} \\
h_{c, i}=h_{c, i-1}+\frac{\dot{Q}}{N \cdot \dot{m}_{c}}
\end{gathered}
$$

For each element of the heat exchanger, the average capacitance rate $(\dot{C})$ for hot and cold stream is calculated according to: 


$$
\dot{C}=\dot{m} \frac{h_{\text {in }}-h_{\text {out }}}{T_{\text {in }}-T_{\text {out }}}
$$

The effectiveness and the dimensionless number of transfer of units (NTU) of each sub-heater for counter flow is calculated respectively as:

$$
\begin{aligned}
\varepsilon_{i} & =\frac{\dot{Q}_{i}}{\dot{C}_{\text {min }}\left(T_{h, \text { in }}-T_{c, \text { in }}\right)} \\
N T U_{i} & =\frac{1}{1-C_{r}} \ln \left(\frac{\varepsilon_{i}-1}{\varepsilon_{i} \cdot C_{r}-1}\right)
\end{aligned}
$$

where $C_{r}$ is the capacitance ratio:

$$
C_{r}=\frac{\dot{C}_{\min }}{\dot{C}_{\max }}
$$

The conductance of each sub-heater is estimated using the above calculated parameters:

$$
U A_{i}=N T U_{i} \cdot \dot{C}_{\min }
$$

\subsubsection{Pressure drop}

The pressure drop is also critical for the PCHE design, because it can crucially affect the s-CO $\mathrm{CO}_{2}$ cycle performance. The pressure losses vary according to the channel type.

The friction losses are calculated with the Darcy-Weisbach equation,

$$
\Delta P=f \frac{L}{D} \frac{\rho V^{2}}{2}
$$

where $L$ is the length of the section. The Darcy friction factor is dependent on the Reynolds number and therefore it is different for each flow regime. For laminar flows with $\operatorname{Re}<2000$ the friction factor is calculated with the Hagen-Poiseuille law,

$$
f=\frac{64}{R e}
$$

While for turbulent flow:

$$
f=0.3164 R e^{-0.25}
$$




\subsection{Dynamic Modeling for PCHE Performance}

For the dynamic study, the thermal mass of the heat exchanger's wall was considered to account for the thermal inertia of the heat exchanger solid plate. During steady-state operation, the amount of heat transferred from the hot fluid is expected to be almost equal to the amount transferred to the cold fluid. However, under transient heating conditions such as shutdown or power-loss of the system, it is expected that the thermal inertia of the heat exchanger should result in time delays between the hot and the cold-side heat transfer rates. The heat exchanger's thermal inertia is estimated as follows:

$$
C_{t h}=m \cdot c_{p}
$$

where the total mass of the heat exchanger is $270 \mathrm{~kg}$ (given by the manufacturer).

The energy conservation equation for the hot and cold fluid and the solid plate are expressed, in order to simulate the heat exchanger's dynamic behavior. To develop the governing equations for transient analysis, the following assumptions were made:

- The fluids are uniformly distributed into the flow channels.

- The wall is in thermal equilibrium and no axial conduction takes place, because of the small thickness of the plates (order of $1.5 \mathrm{~mm}$ ).

- At each node, the calculations are based on average fluid properties.

Therefore, finally, the equation for the hot side is:

$$
\rho_{h} c_{p, h} \frac{\partial T_{h}}{\partial t}+\rho_{h} c_{p, h} u_{h} \frac{\partial T_{h}}{\partial x}=-\left(a \frac{A}{V}\right)_{h}\left(T_{h}-T_{P}\right)
$$

For the cold side:

$$
\rho_{c} c_{p, c} \frac{\partial T_{c}}{\partial t}+\rho_{c} c_{p, c} u_{c} \frac{\partial T_{c}}{\partial x}=-\left(a \frac{A}{V}\right)_{c}\left(T_{P}-T_{c}\right)
$$

For the solid plate:

$$
\rho_{P} c_{p, P} \frac{\partial T_{P}}{\partial t}=\alpha_{h}\left(\frac{A}{V}\right)_{P, h}\left(T_{h}-T_{P}\right)-a_{c}\left(\frac{A}{V}\right)_{P, c}\left(T_{P}-T_{c}\right)
$$

where the ratio $\mathrm{A} / \mathrm{V}\left(\mathrm{m}^{2} / \mathrm{m}^{3}\right)$ indicates the ratio of the surface area in contact with the fluid to the volume of a unit cell for each fluid side or the solid plate. It is considered that the ridge width is small and the thermal conductivity of the plate material is relatively large, therefore the heat conduction resistance of the edges 
between channels was neglected. Furthermore, the heat transfer coefficient and temperature of the fluid are calculated from the average node enthalpy and pressure.

For steady-state simulations, all time derivatives and time dependent terms in the equations are equal to zero. Transient operation analysis requires the time derivatives in order to simulate the gas thermal dynamic effects. The capability to approximate the effects of transient events was built into a model using a quasisteady approach, so the steady state solver is run for each time step, corresponding value of the transient variables.

For the dynamic performance prediction, a Matlab-Simulink model was used, employing the Thermolib library to estimate the thermodynamic properties of $\mathrm{CO}_{2}$ for each time step. Thermolib uses Peng-Robinson equation of state for $\mathrm{CO}_{2}$ properties calculation, which would cause problems if the performance prediction was considering the pseudo-critical region. However, the heat exchanger studied in a region far form critical point, where Peng-Robison and Span-Wagner equations of state seem to present good agreement, as was shown in Chapter 3.1.

\subsection{Numerical Method}

The investigation of the flow phenomena depicting the printed-circuit heat exchanger performance, require the solution of a set of non-linear partial differential equations. This set of equations that describes the fluid dynamics is known as Navier-Stokes equations and their numerical solution gives an understanding of the flow phenomena in three-dimensions. However, the unsteadiness of the three-dimensional flow requires assumptions and models in order to reduce the complexity and the computational time required for the calculations. The development of turbulence models and numerical schemes have helped significantly in this direction. The equations that govern the flow-field are the conservation of mass (continuity equation), the momentum equation and the energy equation. These equations can be expressed either in integral (conservative) or in differential form. The integral form is defined within a control volume with specific size that an external observer considers fixed. Mass, energy and heat can be exchanged through the control surface that encloses the control volume. The differential form can be directly obtained from the integral, if the dimensions of the control volume are considered infinitesimal. The differential form is more common, referring to CFD analyses, so the equations that describe the working fluid are expressed in this form.

Continuity equation:

$$
\frac{\partial \rho}{\partial t}+\nabla \cdot(\rho U)=0
$$


Momentum equation:

$$
\begin{gathered}
\frac{\partial(\rho U)}{\partial t}+\nabla \cdot(\rho U \times U)=-\nabla p+\nabla \cdot \tau+S_{M} \\
\tau=\mu\left(\nabla U+(\nabla U)^{T}-\frac{2}{3} \delta \nabla \cdot U\right)
\end{gathered}
$$

Energy equation:

$$
\frac{\partial\left(\rho e_{0}\right)}{\partial t}-\frac{\partial p}{\partial t}+\nabla \cdot\left(\rho U e_{0}\right)=\nabla \cdot(\lambda \nabla T)+\nabla \cdot(U \cdot \tau)+U \cdot S_{M}+S_{E}
$$

The terms $\mathrm{U}$ is the flow velocity, $\rho$ is the flow density, $\mathrm{p}$ is the pressure, $e_{0}$ is the total inner energy of the flow, $\lambda$ is the volume viscosity and $t$ is the time. The term $\nabla \cdot(U \cdot \tau)$ represents the work done by the viscous stresses and is called viscous work term. This term expresses the heat production in the fluid because of the viscous stresses and in most cases is considered negligible. The term $U \cdot S_{M}$ represents the momentum work done because of external factors. For this analysis, no body or other external forces act on the system, so $S_{M}$ is neglected. Moreover, for the supercritical carbon dioxide that was investigated for this thesis, all the thermodynamic properties were estimated based on the Span-Wagner model, presented in Chapter 3.

The software used for this study is Ansys CFX, as it is suitable to model flows in closed ducts and channels, as the flow in PCHE channels. The entire process of the CFD analysis includes five general steps: (i) three-dimensional geometry definition (designed with SolidWorks), (ii) meshing (Ansys mesh software from suite is used), (iii) CFX-pre where the carbon dioxide properties were introduced and the boundary conditions were selected, (iv) CFX-solver and (v) CFD-Post, where post-processing tools are available.

Ansys CFX solver that follows the computational grid generation, uses a finite volume method for spatial discretization. The grid is firstly used to construct finite volumes, which are employed to solve the conservation equations for mass, momentum and energy for each one. In order to investigate the flow phenomena in the PCHE channels a three-dimensional model was developed. The model includes two zig-zag channels, one for hot and one for cold fluid with intermediate solid walls. 

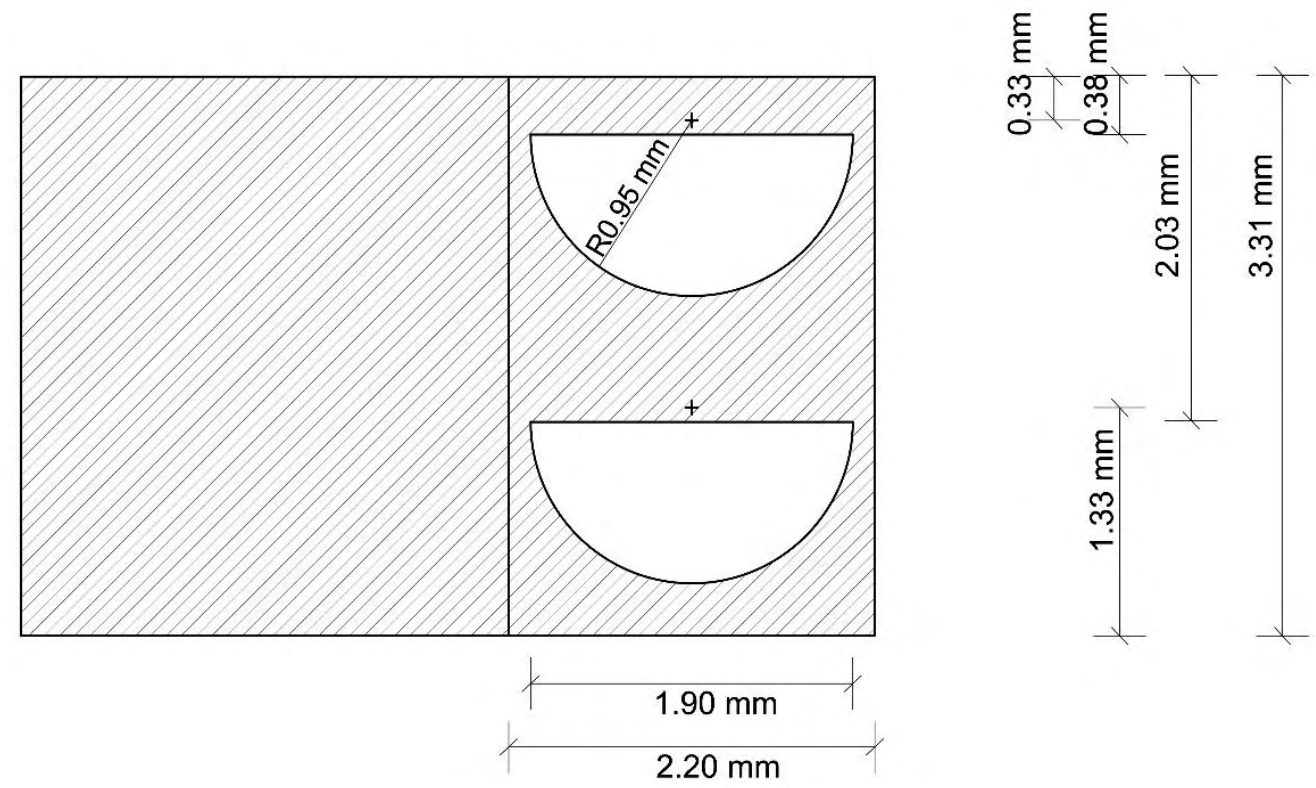

Figure 4.3. Detailed geometry of the designed PCHE channels.

The geometry of the designed channel is presented in Figure 4.3, where the channel diameter, the pitch, the channels thickness and the plate width are presented. The examined heat exchanger length is $54 \mathrm{~mm}$, while the turning angle of the zig-zag passage is 115 degrees (Figure 4.4).

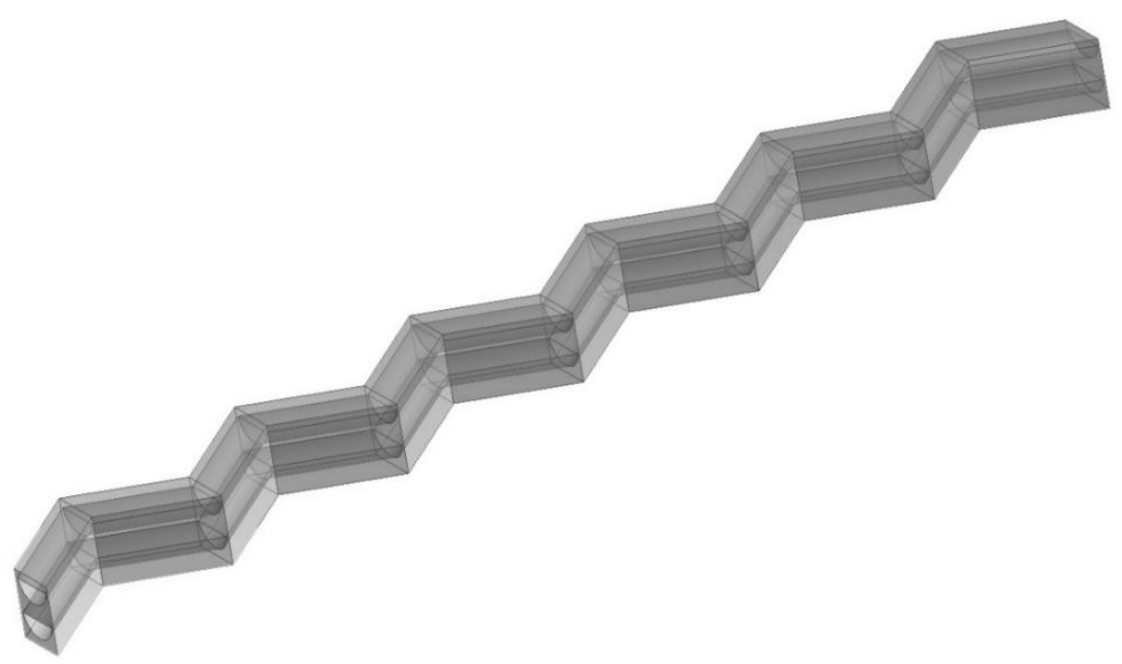

Figure 4.4. Geometry of the whole PCHE developed model. 


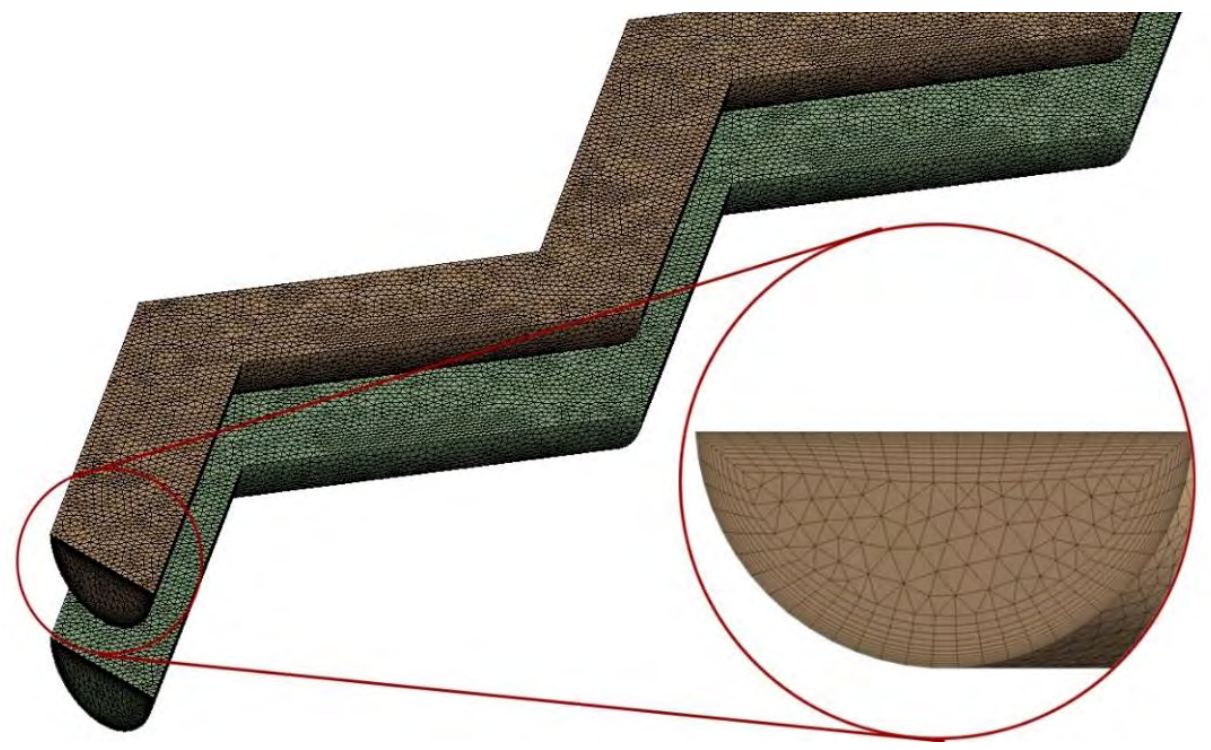

Figure 4.5. Mesh of printed-circuit heat exchanger channels.

An initial mesh was produced and then the mesh was inflated in the boundary, to better catch the boundary layer effects. The final mesh employed is shown in the Figure 4.5, after the mesh independence study. First layer mesh thicknesses were chosen to give a y+ value near 1 . The mesh sensitivity study results converged within 5\%. A total of 389975 nodes (866957 elements) for the $\mathrm{CO}_{2}$ domain, 389488 nodes (863984 elements) for the air domain and 380289 nodes (318000 elements) for the steel domain were employed in the final mesh.

The solid model (heat exchanger module) referenced above was inserted in ANSYS CFX, where a steady state simulation was setup, by employing the conjugate heat transfer mode with two different fluids and the solid part of the heat exchanger made from steel. The location of the boundary conditions and interfaces is illustrated in Figure 4.6. The basic settings for the $\mathrm{CO}_{2}$ part were set with a reference pressure of 80 bar, to minimize errors, while for the estimation of $\mathrm{CO}_{2}$ properties a real gas properties file used, derived from database NIST Refprop. Reference pressure for the air part was set to $10^{5} \mathrm{~Pa}$. A thermal energy heat transfer model was selected. The k-epsilon turbulence model was selected because it was found to better match the thermal behavior of the heat exchanger module (the flow was fully developed and the boundary layer does not affect the solution), as was used also by Baik [62]. The inlet boundary conditions were defined in terms of inlet mass flowrate and temperature, exit pressure, both for the $\mathrm{CO}_{2}$ side and air side. Turbulence intensity at inlet was initiated at $1 \%$. The entire investigation assumed a smooth wall. Periodic boundary conditions were considered for the top, bottom, left and right sides of the steel module. The mass flowrates for each module have been computed by considering a total of 6800 cells for $\mathrm{CO}_{2}$ and air. Reynolds numbers in the heat exchanger module for the nominal operation (design point) were found to be 3762 for the $\mathrm{CO}_{2}$ stream and 3489 for the air stream, respectively. 


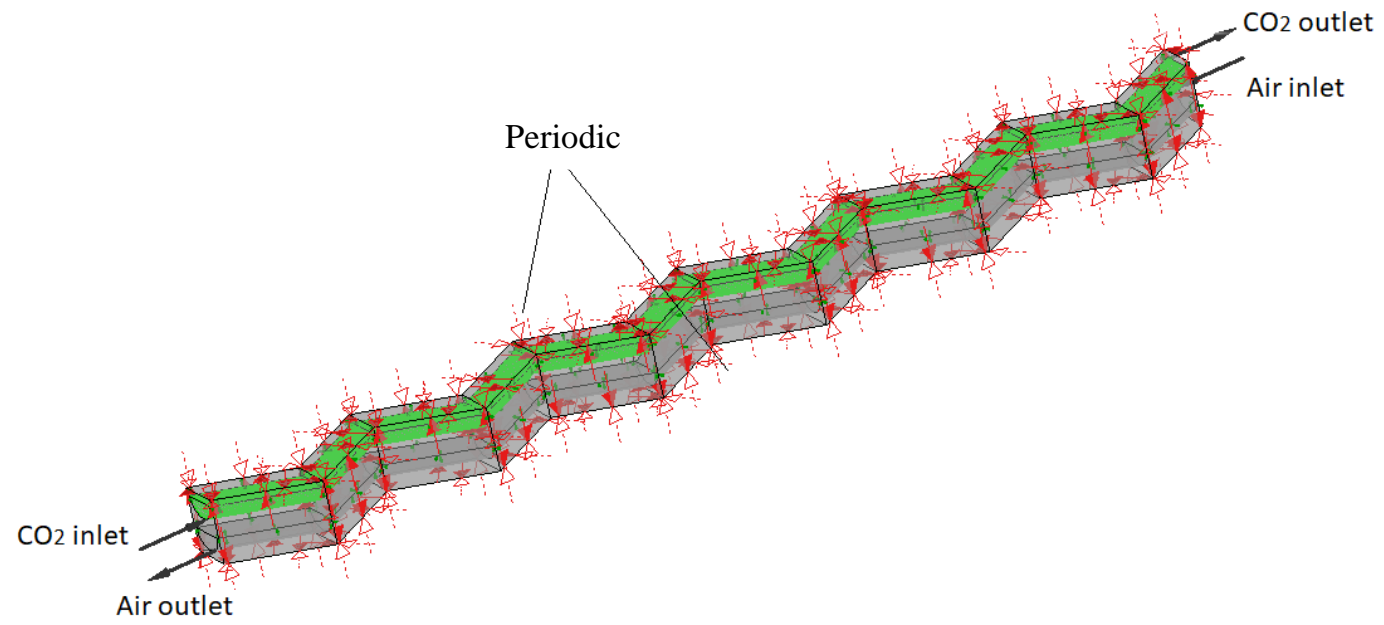

Figure 4.6. Boundary conditions for $\mathrm{CO}_{2}$ and air side.

\subsection{Experimental Method}

A test rig facility has been developed at Cranfield University to enable critical component testing and whole cycle demonstration of a representative waste heat recovery system [99]. Test campaigns associated with this test rig were planned to investigate the behavior of $\mathrm{CO}_{2}$ near the critical point and to examine the control of future cycles by operating and registering the performance of key components under transient performance and comparing the results with numerical simulations.

The test-loop comprises six components including a main heat exchanger, an expansion valve, pre- and post- gas cooler heat exchangers and two transcritical compressors. Figure 4.7 shows a simplified schematic of the conceptual design of the test rig. The main requirement behind this approach was to isolate major equipment components (i.e. heat exchanger) or control loops (i.e. flow split, bypass valves) for testing. A key testing requirement was to maintain a transcritical circulation loop, using different expansion devices to adjust the low pressure level and mass flow in each branch. The circulation loop is a modification of a transcritical $\mathrm{CO}_{2}$ refrigeration system, which can be adjusted to provide a reliable source of carbon dioxide in a supercritical state.

The testing of the main heat exchanger has been split into two steps. Currently, a centrifugal fan provides the required air for testing the main heat exchanger coupled with the aforementioned transcritical compressors (Figure 4.8) (cold-run). At a later stage, the final configuration of the rig is designed to enable fully coupled operation of the centrifugal compression and expansion system together with the main heat exchanger exposed to a hot stream (hot-run). The compression system can deliver the specified mass flow rate of $\mathrm{CO}_{2}$ up to $1 \mathrm{~kg} / \mathrm{s}$. 


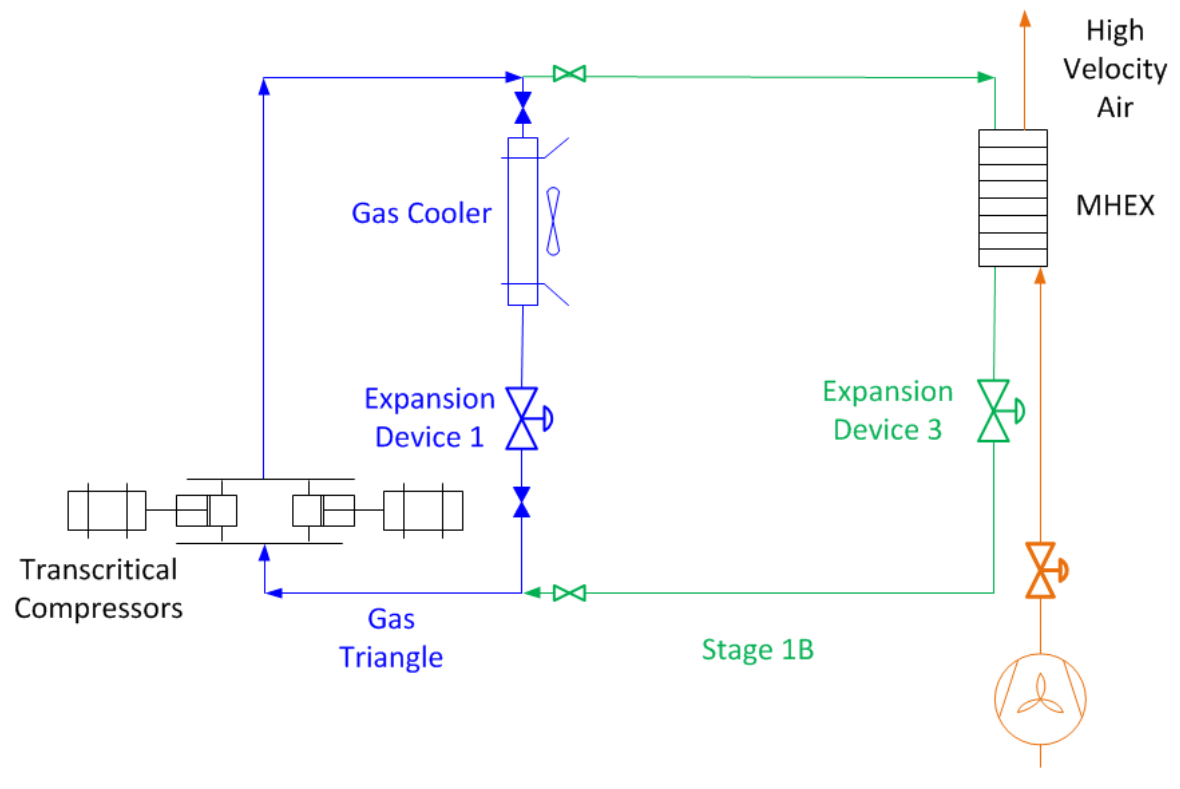

Centrifugal fan

Figure 4.7. Stage development concept of test rig.

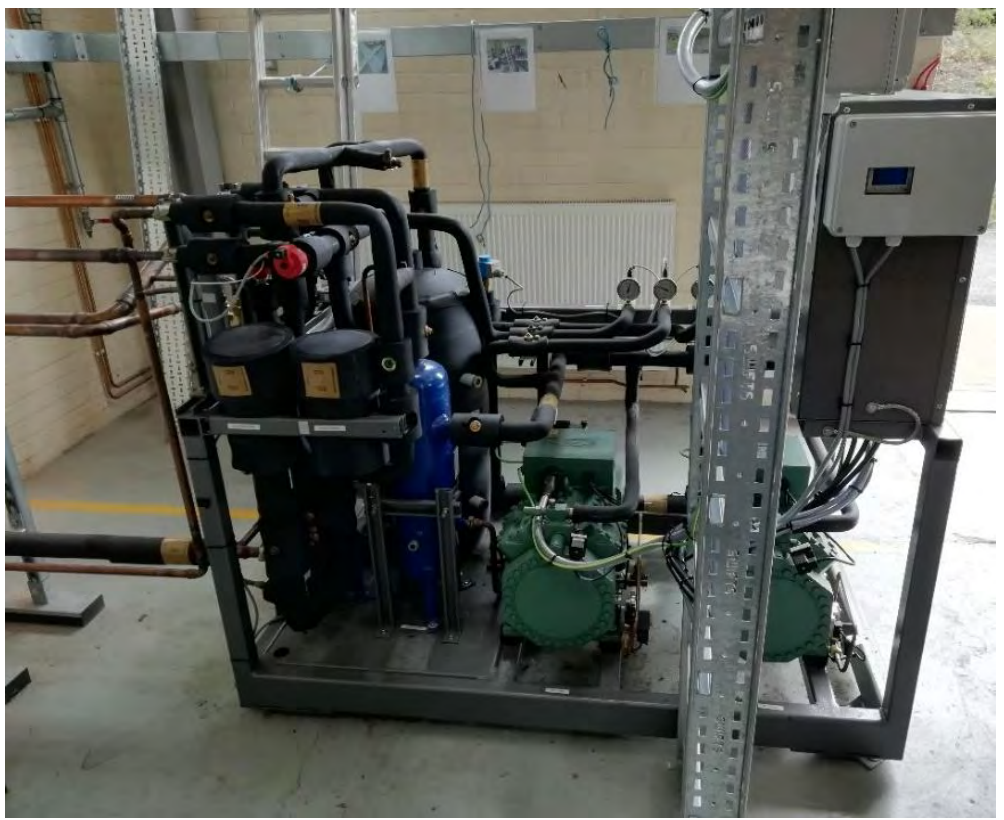

Figure 4.8. Trascritical compressors used for the test facility.

Moreover, the main heat exchanger and the precooler are of the printed-circuit heat exchanger type, designed by Heatric [40], while the post cooler is a typical plate-fin employed in the refrigeration industry. This study intends to present the investigation of the main heat exchanger performance, so the measurement equipment was installed around this component. The design parameters of the cycle are presented in Table 4.1. 
Table 4.1. Design parameters for the $\mathrm{s}-\mathrm{CO}_{2}$ rig test facility.

\begin{tabular}{cc}
\hline Parameter & Value \\
\hline Overall Pressure Ratio & 2.66 \\
Top Pressure $(\mathrm{MPa})$ & 12 \\
Top Temperature $(\mathrm{K})$ & 440 \\
Inlet Compressor Temperature $(\mathrm{K})$ & 294 \\
$\mathrm{CO}_{2}$ mass flow $(\mathrm{kg} / \mathrm{s})$ & $<1$ \\
\hline
\end{tabular}

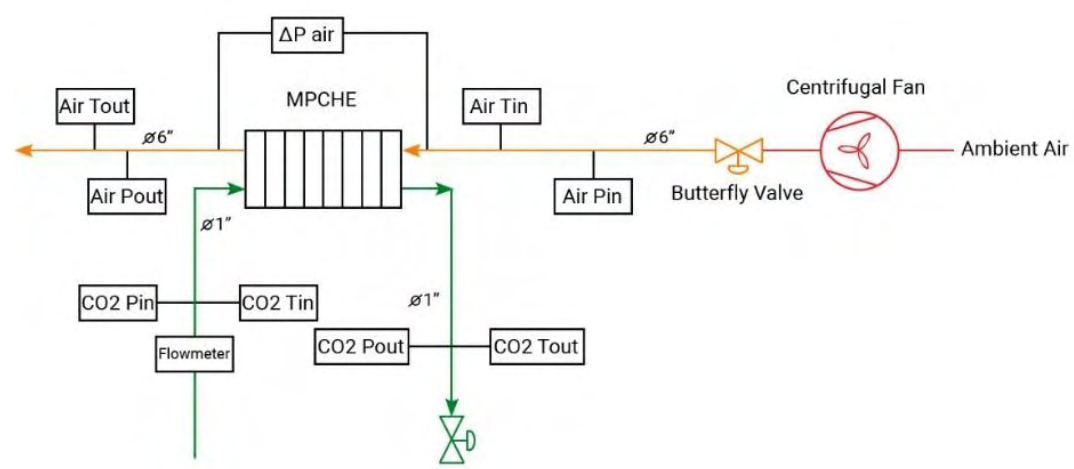

Figure 4.9. Piping and instrument diagram of the experimental setup.

The MPCHE prototype tested in 2018 is a modification of a traditional PCHE (Figure 4.9). The equipment tested at Cranfield comes from a deep-etch development, an improvement in the manufacturing technology suitable for high pressure systems. Testing follows guidelines from the ASME PTC 12.5 [100]. The printed-circuit heat exchanger was based on a special support and the remaining test equipment laid out according to the schematic diagram of Figure 4.10. The MPCHE was connected in counter flow and measurement equipment allowed for the validation of energy balance between the hot and the cold side. Platinum RTD sensors "Class A" $( \pm 0.2 \mathrm{~K})$ were installed at inlet and outlet of MPCHE for both $\mathrm{CO}_{2}$ and air sides according to the operating temperature range, while pressure transducers were installed at each side too. The carbon dioxide temperature and pressure are measured at a distance approximately $10 \mathrm{~cm}$ (equals almost 3 tube diameters) upstream from the respective inlet and downstream the outlet of both MPCHE's. Moreover, a differential pressure gauge was attached to the air side, in order to measure the pressure drop through the heat exchanger for the cold side. Mass flow rates of both sides are controlled by valves, while a Coriolis mass flow meter $( \pm 0.1 \%)$ was installed to measure the $\mathrm{CO}_{2}$ mass flow rate. A hot-wire anemometer RS - 1340 was employed to measure air velocity at a specified position in the air duct and thus estimate air mass flow rate. A centrifugal Alcosa $22142 \mathrm{Fan}\left(1000 \mathrm{ft}^{3} / \mathrm{min}\right.$ nominal capacity) was used to provide cold air to the heat exchanger, while varying a manifold the mass flow was changing. 


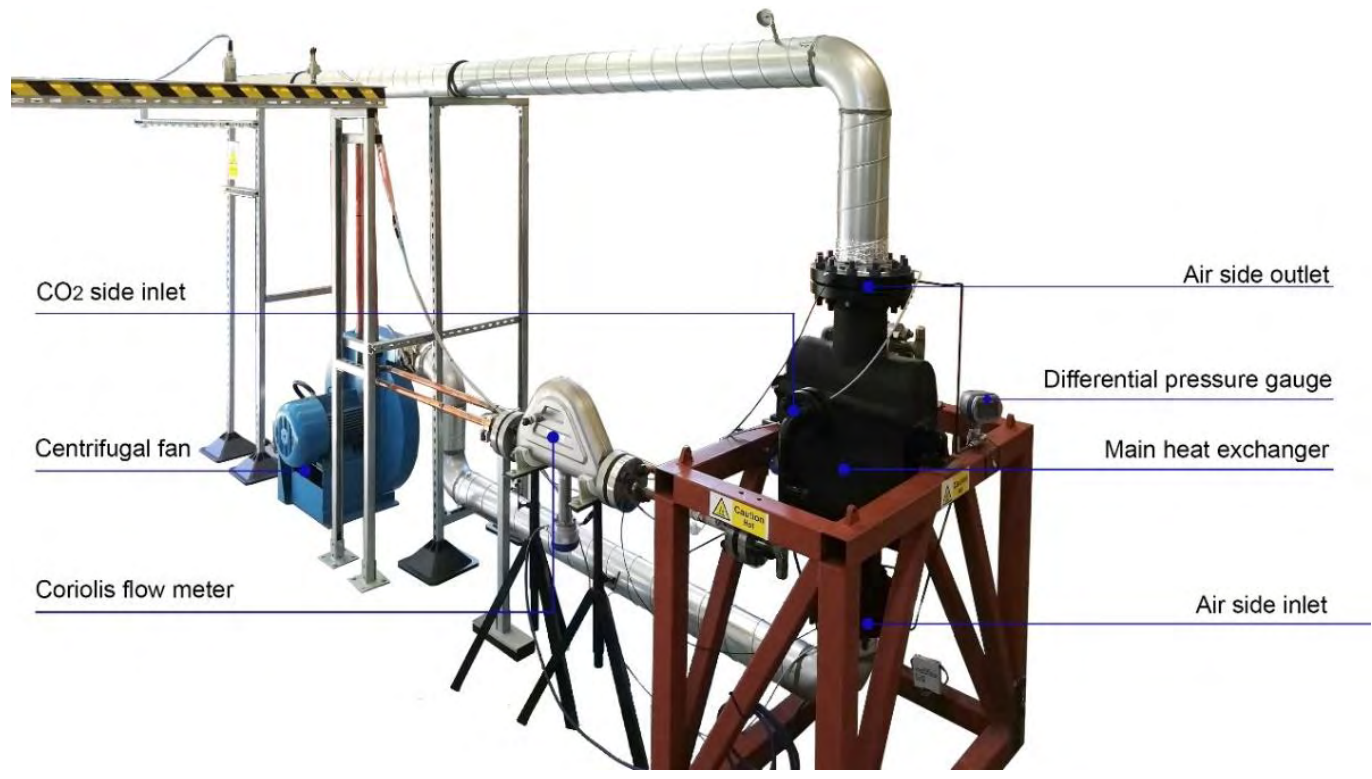

Figure 4.10. Components of experimental test rig for PCHE.

Table 4.2 presents the main characteristics of the tested heat exchanger, and Figure 4.12 and Figure 4.13 show the dimensions and the main views of the tested heat exchanger. Table 4.3 summarizes accuracy details about the detailed instrumentation. Finally, the tubes (Figure 4.11) and the fittings that were used, are made of high-strength copper alloy (Wieland K65), which is a high strength material that permits operating pressures up to $12 \mathrm{MPa}$. The composition of K65 allows silver (hard) brazing to be used in system construction without restrictions.

Table 4.2. Main characteristics of the tested MPCHE.

\begin{tabular}{ccc}
\hline Characteristic & Unit & Value \\
\hline Core dimensions & $\mathrm{mm}$ & $232 \times 366 \times 427$ \\
Mass operating & $\mathrm{kg}$ & 270 \\
Area provided & $\mathrm{m}^{2}$ & 12.7 \\
Design heat load & $\mathrm{kW}$ & 45.7 \\
\hline
\end{tabular}

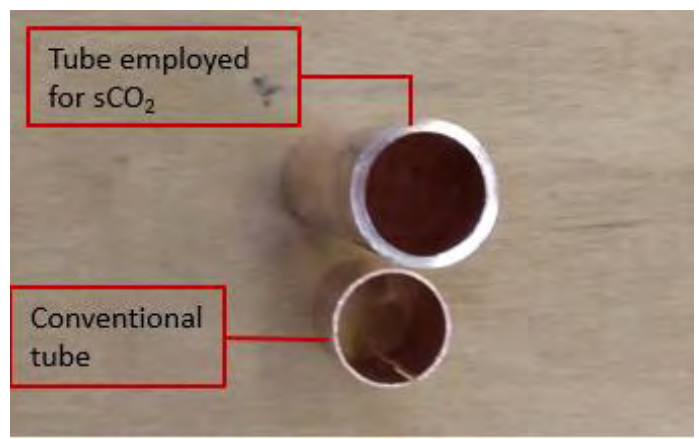

Figure 4.11. Difference between tubes employed for conventional and $\mathrm{s}-\mathrm{CO}_{2}$ applications. 


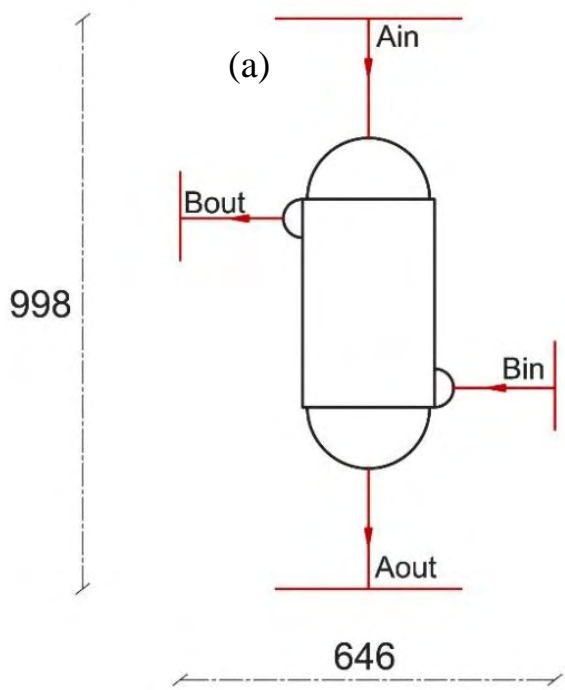

(b)

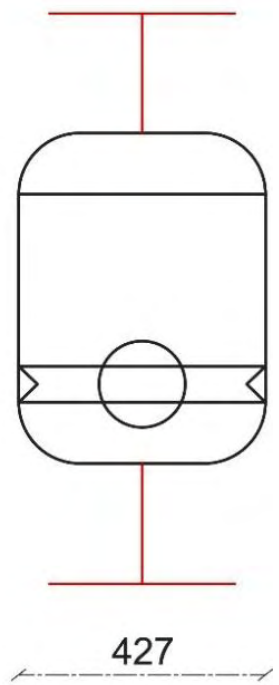

Figure 4.12. Schematic diagram of the (a) front and (b) side view of the tested PCHE.
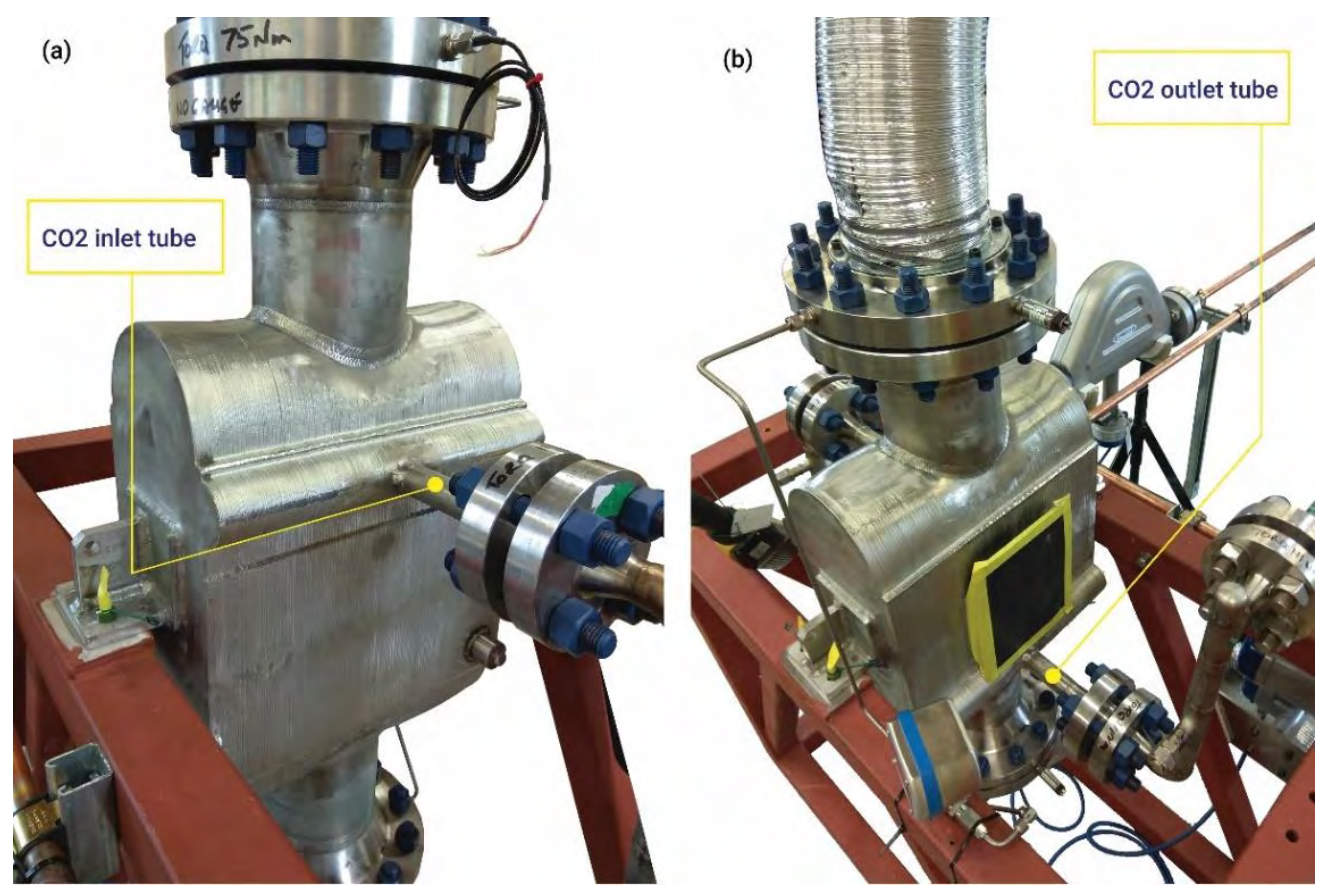

Figure 4.13. View of the (a) inlet and (b) outlet side of the tested PCHE. 


\subsubsection{Uncertainty analysis}

Knowing the experimental uncertainty, associated to a measurement, allows the comparison to be made with the same measurement taken under different circumstances. The measurement of the same quantity by means of the same equipment but in different conditions will not produce probably the same results. Differences are due to variations in the calibration, environmental effects and human factors. The results of a repeated experiment are nevertheless expected to remain within the interval around each measurement point. This interval is referred to as the experimental uncertainty. It is a measure of possible errors made on particular experiment. From the experiments conducted in the facility, raw data of temperature, pressure, flow rate and differential pressure between streams of MPCHE are collected. Because of the rapidly changing properties of $\mathrm{CO}_{2}$ near the critical point, the obtained raw data required post-processing.

The accuracy of measurements of facility's sensors is presented in Table 4.3. Because of this abrupt variation close to the critical point of carbon dioxide, small uncertainty value of the measurement could result in a considerable uncertainty near the critical point.

The procedure that must be followed in order to obtain the overall uncertainties is presented in the GUM (Guide to the expression of Uncertainty in Measurements) method [101]. For this study the uncertainty analysis followed by Stefopoulos et al. [102]. The calculation of the heat transfer in the printed-circuit heat exchanger has to take into consideration the uncertainty in the measured mass flow rate, temperature and pressure.

Table 4.3. Key instrumentation characteristics.

\begin{tabular}{cccc}
\hline Parameter & Device & Range & Accuracy \\
\hline Pressure & Piezo resistive & & \\
Temperature & RTD Pt100 & $0-20 \mathrm{MPa}$ & $\pm 0.25 \%$ \\
Mass flow & Coriolis & $-20-250{ }^{\circ} \mathrm{C}$ & $\pm 0.2{ }^{\circ} \mathrm{C}$ \\
\hline \multicolumn{2}{c}{ Air Stream } & $0-1 \mathrm{~kg} / \mathrm{s}$ & $\pm 0.35 \%$ \\
\hline Pressure & Piezo resistive & $0-0.1 \mathrm{MPa}$ & $\pm 0.25 \%$ \\
Diff. pressure & Piezo resistive & $0-75 \mathrm{mbar}$ & $\pm 0.3 \%$ \\
Temperature & RTD Pt100 & $0-150{ }^{\circ} \mathrm{C}$ & $\pm 0.2{ }^{\circ} \mathrm{C}$ \\
Velocity & Hot wire Anemometer & $0-40 \mathrm{~m} / \mathrm{s}$ & $\pm 1 \%$ \\
\hline
\end{tabular}


The equation for the heat transfer is expressed as:

$$
\frac{\delta \dot{Q}}{\dot{Q}}=\left[\left(\frac{\delta \dot{m}}{\dot{m}}\right)^{2}+\left(\frac{\delta h_{\text {in }}}{h_{\text {out }}-h_{\text {in }}}\right)^{2}+\left(\frac{\delta h_{\text {out }}}{h_{\text {out }}-h_{\text {in }}}\right)^{2}\right]^{1 / 2}
$$

where

$$
\begin{gathered}
\delta h_{\text {in }}=\left[\left(\frac{\partial h_{\text {in }}}{\partial T_{\text {in }}} \delta T\right)^{2}+\left(\frac{\partial h_{\text {in }}}{\partial P_{\text {in }}} \delta P\right)^{2}\right]^{1 / 2} \\
\delta h_{\text {out }}=\left[\left(\frac{\partial h_{\text {out }}}{\partial T_{\text {out }}} \delta T\right)^{2}+\left(\frac{\partial h_{\text {out }}}{\partial P_{\text {out }}} \delta P\right)^{2}\right]^{1 / 2}
\end{gathered}
$$

Therefore, from the measured values and the accuracy of sensors for the mass flow rate, temperature and pressure, the uncertainty of both hot and cold sides can be calculated. The partial derivatives for the accuracy estimation of enthalpy were estimated using the Nist Refprop database [103].

The pressure drop of the PCHE is measured with the differential pressure gauge of the facility for the air-side and pressure transmitters for s- $\mathrm{CO}_{2}$ side. The pressure drop of the PCHE comes from the headers and the channels of the heat exchanger.

$$
\Delta P_{P C H E}=\Delta P_{c h}+\Delta P_{\text {header, }, \text { in }}+\Delta P_{\text {header, }, \text { out }}
$$

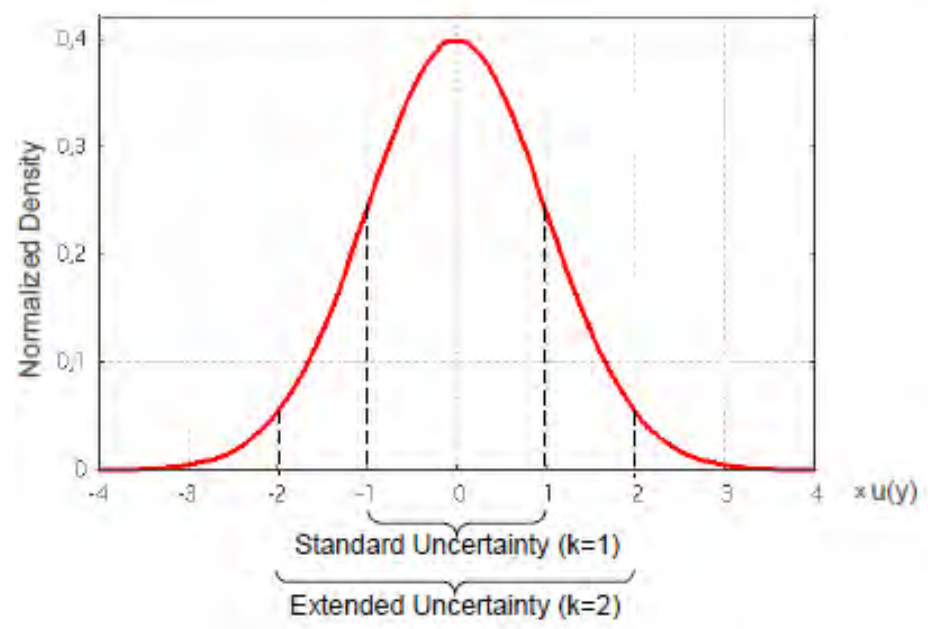

Figure 4.14. Normalized normal distribution of uncertainty for standard (66\% level of confidence) and extended uncertainty (95\% level of confidence) [104]. 
The pressure drop of the heat exchanger is measured with the differential pressure gauge, and the pressure drop for the inlet and outlet headers are calculated with loss factors. Each pressure drop includes the uncertainty of each instrument.

The relative uncertainty in the measured and calculated pressure drop is expressed as:

$$
\frac{\sigma_{\Delta P_{c h}}}{\Delta P_{c h}}=\left[\frac{\sigma_{\Delta P_{P C H E}}^{2}+\sigma_{\Delta P_{\text {header }, \text { in }}}^{2}+\sigma_{\Delta P_{\text {header }, \text { out }}}^{2}}{\left(\Delta P_{P C H E}-\Delta P_{\text {header }, \text { in }}-\Delta P_{\text {header }, \text { out }}\right)^{2}}\right]^{1 / 2}
$$

where $\sigma_{\Delta P}$ represents the standard deviation of each pressure drop, as the uncertainty follows a normal distributed interval with a $95 \%$ level of confidence.

The header pressure drop is calculated as:

$$
\Delta P_{\text {header }}=\frac{1}{2} K \rho v^{2}=\frac{K \dot{m}^{2}}{2 A^{2} \rho}
$$

where $K$ is the loss coefficient, $\rho$ is the density of fluid, $v$ is the velocity of the fluid, $\dot{m}$ is the mass flow rate ad $\mathrm{A}$ is the tube area.

So, the standard deviation of the header pressure drop is calculated as:

$$
\begin{gathered}
\sigma_{\Delta P_{\text {header }}}=\left[\left(\frac{\partial(\Delta P)}{\partial \dot{m}}\right)^{2} \sigma_{\dot{m}}^{2}+\left(\frac{\partial(\Delta P)}{\partial A}\right)^{2} \sigma_{A}^{2}+\left(\frac{\partial(\Delta P)}{\partial \rho}\right)^{2} \sigma_{\rho}^{2}\right]^{1 / 2} \\
\frac{\sigma_{\Delta P_{\text {header }}}}{\Delta P_{\text {header }}}=\left[\left(2 \frac{\delta \dot{m}}{\dot{m}}\right)^{2}+\left(4 \frac{\delta D}{D}\right)^{2}+\left(\frac{\delta \rho}{\rho}\right)^{2}\right]^{1 / 2} \\
\delta \rho=\left[\left(\frac{\partial \rho}{\partial T} \delta T\right)^{2}+\left(\frac{\partial \rho}{\partial P} \delta P\right)^{2}\right]^{1 / 2}
\end{gathered}
$$

Thus, the relative uncertainty of the pressure drop is calculated with the form loss coefficient, the accuracy of sensors and the measured values of mass flow, temperature and pressure. 


\subsection{Conclusions}

This chapter presents the methods that have been employed for the design and analysis of the printed-circuit heat exchangers. Firstly, the selection of heat exchangers design is discussed based on the operating conditions and the system requirements. Then, the methods employed for the steady-state one-dimensional analysis are presented in order to estimate the heat transfer coefficient and pressure drop for straight and wavy channels of heat exchangers, while the dynamic modeling of a PCHE is also discussed. Consecutively, the numerical method used is analyzed, with the employing compressible flow equations (Navier-Stokes), the turbulence model, the developed geometry model and the meshing of the model. Finally, the experimental method is explained, presenting the experimental facility, the sensors type and the test cases that were simulated, while the uncertainty analysis method is also discussed. 


\section{Chapter 5}

\section{Centrifugal Compressor Design Methodology}

Supercritical $\mathrm{CO}_{2}$ cycles include compressors operating near the critical point, resulting in compact power-dense machinery and introducing plenty of challenges. Centrifugal compressors are a key component of the supercritical carbon dioxide cycles, as it operates in the pseudo-critical region of the carbon dioxide, where the real gas effects are significant. This section presents a centrifugal compressor design method for working supercritical carbon dioxide. The elements consisting the centrifugal compressor and the adopted design approach are presented. The design and analysis of the compressor take place with both one-dimensional and three-dimensional methods. Firstly, the direct problem for a known geometry compressor is presented, employing equations applied to turbomachinery and appropriate pressure loss models. Then, the inverse problem is considered, employing the same procedure but in a way that the geometry is the model output for known inlet and outlet conditions. Finally, the three-dimensional governing equations of the flow and the appropriate models for their solution are presented. The simulations results are presented in Chapter 7.

\subsection{One-Dimensional Compressor Model}

A radial configuration for the main compressor is designed due to its lower volume flow and wider range to facilitate variation in gas properties due to operation near the critical point. A one-dimensional design tool is developed in order to model the performance of the centrifugal compressor, incorporating pressure loss coefficients and performance parameters based on literature. The process was derived in two parts: a direct and an inverse problem, as was proposed by Monge [79]. Firstly, the direct problem is built for a known geometry of a centrifugal compressor used by Sandia National Laboratories, resulting in outlet conditions that were validated by Sandia results. Therefore, the design model was proved to predict with a good accuracy the compressor performance, the indirect problem was solved. For the indirect problem, the thermodynamic conditions derived by the preceded cycle's thermodynamic analysis, were used in order to define the final geometry of the compressor. Then, a three-dimensional geometry 
is derived from the 1D model results and a computational fluid dynamic analysis takes place in order to examine the s- $\mathrm{CO}_{2}$ flow in the compressor.

\subsubsection{Direct problem}

The developed model for the direct problem uses as input known published data by Sandia such as the total inlet conditions, the mass flow rate, the shaft speed and some geometrical data. Using these parameters as input, the model estimates the flow conditions at the compressor outlet and performance aerodynamic parameters such as slip factor, tip clearance and wall frictions. The procedure is based on the approach presented by Aungier [78]. The computational procedure includes the development of numerous modules with each one represening a corresponding part of the centrifugal compressor. Each module's outlet is the inlet for the next module. The consequence of the modules is the following: inducer, impeller, vaneless space and vaned diffuser. In this section, the subscripts from 1 to 5 are used to indicate the inlet and outlet conditions of inducer, impeller, vaneless space and diffuser respectively. The volute is not included in this study.

The flow changes thermodynamic conditions as it passes through each element of the whole configuration, according to the change of its motion and velocity (Figure 5.1). For the impeller, this change of thermodynamic conditions is defined by the Euler's law.

$$
\Delta h_{o}=\Delta\left(U c_{\theta}\right)=U_{2} c_{\theta, 2}-U_{1} c_{\theta, 1}
$$

The fact that the flow presents total pressure losses as it runs through each compressor component, confirms that $\Delta s \geq 0$ affirming the second law of Thermodynamics for the system. All this procedure is expressed in the following enthalpy-entropy diagram (Figure 5.1).

However, the enthalpy change is not represented by the Euler equation, but subtracting all the internal losses [78] from the Euler enthalpy change $\Delta h_{o}$. The internal losses, which come from the non-ideal behavior of the flow, include the following losses:

- Incidence losses

- Friction losses

- Aerodynamic losses (incorporating blade loading)

- Tip clearance losses

- Mixing losses

Apart from the internal losses, there are losses that rise the stagnation enthalpy at the outlet without any corresponding increase in pressure, and are referred as external or parasitic losses [74] and represent the mechanical deficiencies in the impeller. The parasitic losses comprise: 


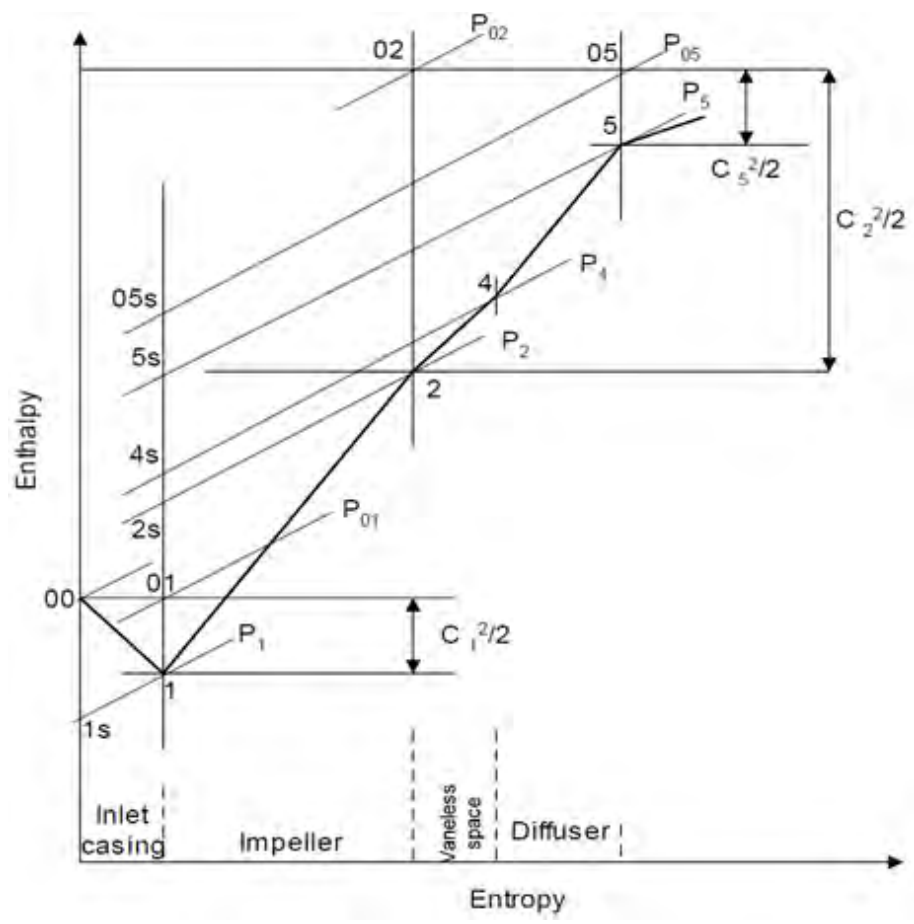

Figure 5.1. Enthalpy-entropy diagram for the centrifugal compressor stage.

- Disk friction (windage) losses

- Recirculation losses

- Leakage losses

The developed model estimates the relative total pressure loss correlations to account for internal losses and work loss correlations to account for parasitic losses. Finally, based on the amount of these enthalpy losses, the efficiency of the impeller is estimated.

\subsubsection{Inducer}

The inducer is used to accelerate the fluid from absolute velocity $c_{1}$ to $c_{2}$ and decrease the static pressure $\left(P_{1}>P_{2}\right)$. Consequently, the static enthalpy is decreased, while the total enthalpy is kept constant $\left(h_{1}>h_{2}, h_{01}=h_{02}\right)$. Due to the vicinity to the critical point, care must be taken to avoid that the fluid is decelerated up to the two phase region, preventing condensation.

From the continuity equation at the inducer inlet:

$$
\dot{m}=\rho_{1} c_{1} A_{1}
$$

where the absolute velocity $c_{1}$ can be calculated only if the inlet area of the inducer $A_{1}$ is known. 
The area is calculated by means of the impeller outlet tip radius $r_{t}$ obtained in the preliminary design, which in this section will be indicated as $r_{3 t}$ (outlet of the impeller). The inducer is modelled assuming its geometry similar to that in Figure 2.6. This means that the hub radius varies reducing the area from $A_{1}$ to $A_{2}$, while the tip radius at inlet and outlet of inducer is kept constant leading to $r_{1 t}=r_{2 t}$. The area $A_{1}$ is obtained by solving the following system of equation:

$$
\begin{gathered}
A_{1}=\pi \cdot\left(r_{1 \text { tip }}^{2}-r_{1 h u b}^{2}\right) \\
A_{2}=(1-B) \cdot \pi \cdot\left(r_{2 t i p}^{2}-r_{2 h u b}^{2}\right) \\
K_{1}=r_{3 t i p} / r_{2} \\
K_{2}=r_{2 t i p} / r_{2 h u b}
\end{gathered}
$$

Where $K_{1}$ and $K_{2}$ are two geometric inputs defined as the ratio between impeller outlet tip radius (values in literature between 1.6 and 3.3), $r_{2}$ expresses the mean impeller inlet radius and $B$ is the blockage factor, a measure of the deviation of the flow from axisymmetry, and the empirical value for simple axial inducer inlets used in this study is 0.02 [77].

\subsubsection{Incidence losses}

By estimating the absolute velocity at inducer inlet, the total operating properties can be calculated. In order to calculate the outlet conditions of the inducer, the pressure loss coefficient has to be estimated. There are two kinds of losses in the inducer, the incidence and the contraction losses. The incidence losses are due to the difference between the relative component of the velocity and the blade angle at the leading edge. Conrad et al. [105] has proposed a loss model $\left(\Delta h_{i n c}=f_{i n c} \frac{w_{L}^{2}}{2}\right)$, where $W_{L}$ is the difference in relative swirl velocity between the flow entering at the optimum blade flow angle and the real one, and $f_{\text {inc }}$ is a dimensionless parameter ranging from 0.5 to 0.7 when experimental correlations are not available.

However, the coefficient that expresses the incidence losses for this study is given by Aungier [78], as an alternative of an experimental that is in use for working air.

$$
\omega_{\text {incidence }}=\left(1-\frac{c_{2}}{w_{2} \cos \beta_{2}}\right)^{2}+\left(\frac{Z_{b} t_{b}}{2 \pi r_{2} \cos \beta_{2}}\right)
$$




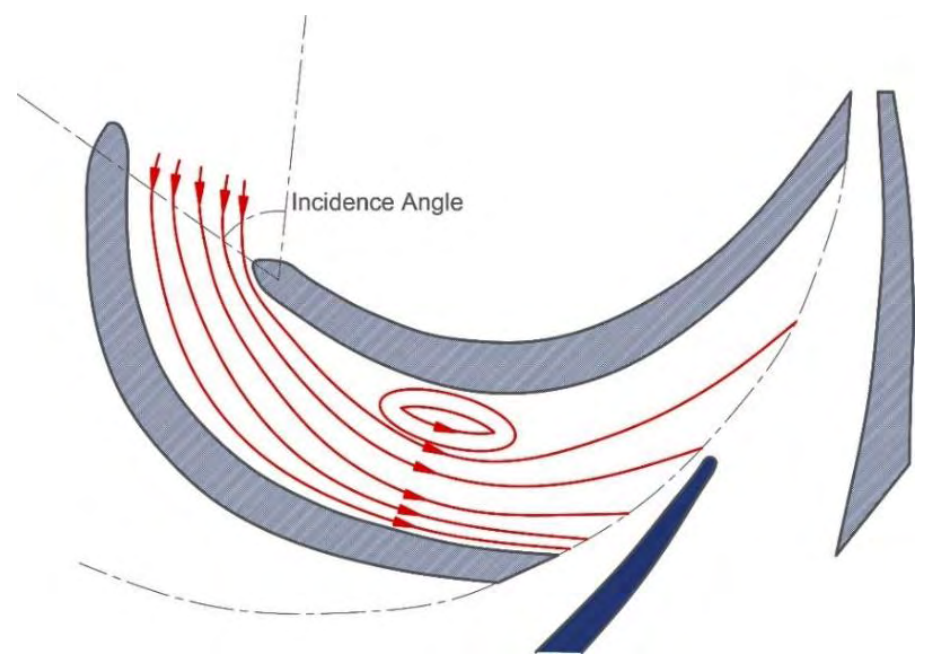

Figure 5.2. Impeller cross section, where incidence losses present.

On the other hand, the contraction losses are considered to be the result of the flow passage area reduction $A_{1}$ to $A_{2}$ and they are estimated:

$$
\omega_{\text {contr }}=\left(1-\frac{A_{2}}{A_{1}}\right)^{2}
$$

For the inducer the pressure loss coefficient $\omega_{\text {inducer }}$ is defined as:

$$
\omega_{\text {inducer }}=\omega_{\text {incidence }}+\omega_{\text {contr }}
$$

Defining this pressure loss coefficient, the outlet pressure of the inducer can finally be defined through the following equation:

$$
P_{02}=P_{01}-\omega_{\text {inducer }}\left(P_{01}-P_{1}\right)
$$

\subsubsection{Impeller}

The most critical and geometrically complex component of the compressor is the impeller. The impeller is used to increase the energy content of the fluid by increasing its angular momentum. It also compresses the flow from pressure $P_{2}$ to pressure $P_{3}$ and due to its rotating nature, it is analyzed in a relative frame of reference, which rotates with the impeller shaft. So, the total conditions are estimated using the relative component of velocity through the rothalpy definition:

$$
I=h_{o, r}-\frac{1}{2} U^{2}=h+\frac{w^{2}}{2}-\frac{U^{2}}{2}
$$


As the flow is analyzed in the relative frame of reference, the loss coefficients apply to the relative dynamic pressure across the impeller. The impeller losses are classified as internal, due to irreversibilities produced by the flow behavior, and parasitic, which come from the mechanical losses which result in a loss of enthalpy increase and in turn total pressure losses. The developed model is based on the pressure loss coefficients for the impeller, incorporating five losses contributions: blade loading coefficient $\omega_{B L}$, aerodynamic loss coefficient $\omega_{H S}$, mixing losses $\omega_{\text {wake }}$ and $\omega_{\Lambda}$, wall friction losses $\omega_{F L}$ and clearance losses $\omega_{C L}$.

The impeller design begins with the definition of some geometrical data, such as the axial length and the impeller length, the blade height, the outlet radius of the impeller, etc. The number of main blades $\left(Z_{b}\right)$ and the number of splitter blades has been defined from the start of the procedure. The above mentioned geometrical parameters are defined by the following equations:

$$
\begin{gathered}
L_{\text {imp }}=2 r_{2}(0.08+1.58 \Phi) \\
L_{\text {axial }}=d_{3 t i p} \cdot\left(0.014+0.023 \cdot \frac{d_{3 t i p}}{d_{2 t i p}}+1.58 \Phi\right) \\
h_{b}=\frac{A_{3}}{2 \pi r_{3}} \\
r_{3}=r_{3 t i p}
\end{gathered}
$$

Where the stage flow coefficient for centrifugal compressors is defined by:

$$
\Phi=\frac{c_{3}}{U_{3}}=\frac{\dot{m}}{\rho_{01} \pi r_{3}^{2} U_{3}}
$$

The Aungier correlations proposed are validated for working air with $0.009<\Phi<$ 0.15 and pressure ratios up to 3.5. Although there are no correlations proposed for supercritical compressors this study uses the Aungier, since the values of flow coefficient and pressure ratio are within ranges.

Moreover, the work input coefficient $I_{c}$, defined as the actual work supplied to the flow is introduced:

$$
I_{c}=\frac{\Delta h_{o}}{U_{3}{ }^{2}}
$$

The quantity $\Delta h_{o, B}$ is the actual specific stagnation enthalpy difference over the impeller and it is related to the blade work input coefficient as [106]:

$$
I_{B}=\frac{\Delta h_{o, B}}{U_{3}^{2}}
$$


Aungier relates the blade work input to the flow coefficient $\Phi$ with the following equations:

$$
\begin{gathered}
I_{c}=0.62-\left(\frac{\Phi}{0.4}\right)^{3}+\frac{0.0014}{\Phi} \text { for shrouded impellers } \\
I_{c}=0.68-\left(\frac{\Phi}{0.37}\right)^{3}+\frac{0.002}{\Phi} \text { for unshrouded impellers }
\end{gathered}
$$

The performance estimation of the impeller demands the definition and calculation of two more significant parameters, the slip factor $(\sigma)$ and the distortion factor $(\lambda)$.

The relative flow leaving the impeller of the centrifugal compressor cannot receive all the guidance from the blade, even if totally frictionless conditions are considered, and part of the provided energy will be lost. This difference between the ideal guidance and the real flow is defined as slip, and is described by the slip factor that expresses the ratio between the actual work input and the ideal work in case the flow takes the perfect guidance by the blade [107]:

$$
\sigma=\frac{\Delta h_{o, B}}{\Delta h_{o, B, i d}}=\frac{c_{\theta, 3} U_{3}}{\Delta h_{o, B, i d}}
$$

Many expressions have been proposed in order to express the slip factor in literature. The three most common expressions have been proposed by Stodola (1927), Stanitz (1952) [108] and Wiesner (1967) [109]. In this study the Wiesner's formulation, which comes from experimental results, has been applied:

$$
\sigma=1-\frac{\sqrt{\cos \beta^{\prime}}}{Z_{b}{ }^{0.7}\left(1-\frac{C_{r, 3}}{U_{3}} \tan \beta^{\prime}{ }_{3}\right)}
$$

where $Z_{b}$ is the number of blades and $\beta^{\prime}{ }_{3}$ is the ideal blade angle at exit.

The distortion factor $(\lambda)$ is used in order to model the blockage of the flow. So the definition of the blockage coefficient $B_{3}$ is used in order to define the distortion factor [79]:

$$
\lambda=\frac{1}{1-B_{3}}
$$

Having defined some important performance parameters, the performance estimation follows that takes into account the principal losses. 


\subsubsection{Aerodynamic losses}

Blade loading losses are the result of the diffusion of the relative flow inside the impeller channels, leading in a pressure gradient in the blade-to-blade direction. The high density supercritical $\mathrm{CO}_{2}$ is expected to present a larger resistance in flow deflection than air, hence a larger loss coefficient. Furthermore, the deceleration of the flow through the passage increases the boundary layer thickness which can induce the separation of the flow from the blading [110]. The obvious solution of the number of blades increase, would lead in more significant friction losses [79]. These losses are related to the Diffusion Factor as this parameter relates the degree of diffusion in relative velocity.

These losses are evaluated with the model proposed by Aungier [78], which requires a simple model for the meridional (stream-wise) velocity distribution along the pressure and suction sides of the blades. A simple velocity profile can be obtained from a simple irrotational flow analysis (Figure 5.3).

The blade loading coefficient is calculation as a function of the velocity difference:

$$
\begin{aligned}
\Delta w & =\frac{2 \pi d_{2} U_{2} I_{B}}{Z_{b} L_{i m p}} \\
\omega_{B L} & =\frac{1}{24}\left(\frac{\Delta w}{W_{2}}\right)^{2}
\end{aligned}
$$

The pressure gradient in the impeller channel although dominant is not the only one acting but there is also another pressure gradient from hub to shroud that has to be taken into account in the form of an additional loss term.

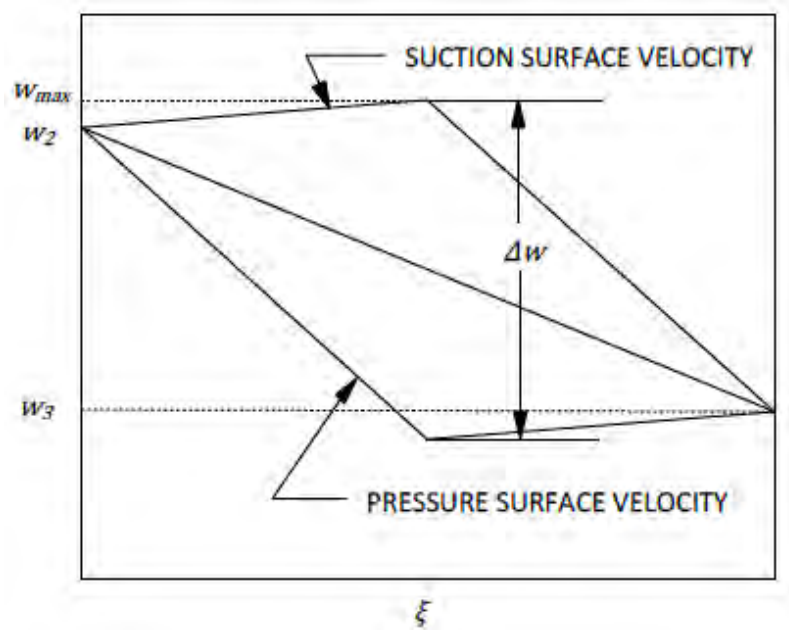

Figure 5.3. Blade loading model [78]. 
The aerodynamic losses from hub to shroud $\omega_{H S}$ depend on the mean channel width $(\bar{l})$ and the velocity profile $(\bar{w})$.

$$
\begin{gathered}
\bar{l}=\frac{\left(r_{2 s}-r_{2 h}\right)+r_{3}}{2} \\
\bar{w}=\sqrt{\frac{w_{2}^{2}+w_{3}^{2}}{2}} \\
\omega_{H S}=\frac{1}{6}\left(\frac{\overline{l w}}{w_{2}}\right)^{2}
\end{gathered}
$$

\subsubsection{Mixing losses}

There are two separate mechanisms that can be developed and provoke mixing losses in the impeller. The first is due to the distorted flow, causing pressure losses and it is a function of the distortion factor previously defined:

$$
\omega_{\lambda}=\left[\frac{(\lambda-1) c_{r 3}}{w_{2}}\right]^{2}
$$

The second loss mechanism is caused because of the mixing process of the wake with the channel flow after the trailing edge and its calculation involves the meridional velocities before and after mixing [78]. The wake velocity is approximated by the separation velocity $w_{\text {sep }}$, which consists the result of the pressure expansion. So, a diffusion limit is assumed, leading in the separation velocity estimation.

$$
\begin{gathered}
w_{\text {sep }}=\left\{\begin{array}{c}
w_{\text {sep }}=w_{3}, \quad \text { for } D_{f} \leq 2 \\
w_{\text {sep }}=\frac{w_{3} D_{f}}{2}, \quad \text { for } D_{f}>2
\end{array}\right. \\
c_{r 3, \text { wake }}=\sqrt{w_{\text {sep }}^{2}-w_{\theta 3}^{2}} \\
c_{r 3, \text { mix }}=c_{r 3} \frac{A_{3}}{\pi d_{3 t} b_{3}}
\end{gathered}
$$

So finally, the loss coefficient is estimated as:

$$
\omega_{\text {mix }}=\left(\frac{c_{r 3, \text { wake }}-c_{r 3, \text { mix }}}{w_{2}}\right)^{2}
$$




\subsubsection{Friction losses}

The wall friction losses are developed due to the shear forces exerted by the fluid on the boundary layer. They are modeled by the well-known Schlichting model [83], based on a friction factor coefficient $c_{f}$ estimated as a function of Reynolds number, averaged at the inlet and outlet of the duct, and relative roughness $\left(\varepsilon / d_{h}\right)$ that is considered for simplicity 0.001 . The wall friction coefficient is a function of the hydraulic diameter $d_{h}$, of the impeller length $L_{i m p}$ and the relative velocity:

$$
\omega_{f r}=4 c_{f} \frac{L_{i m p}}{d_{h}}\left(\frac{\bar{w}}{w_{2}}\right)^{2}
$$

\subsubsection{Tip clearance losses}

The tip clearance losses occur because of the negative pressure gradient at the two sides of the blades and the gap that exists between the impeller and the shroud. The fluid that flows through the gap is not exerting any work to the blade, leading in an energy and pressure loss.

The tip clearance coefficient $\omega_{c l}$ requires estimating both the pressure difference across the gap that the leak happens $\Delta p_{C L}$ and the mass flow that leaks $\dot{m}_{C L}$ :

$$
\begin{gathered}
\Delta p_{C L}=\frac{\dot{m}\left(r_{3} c_{\theta, 3}-r_{2} c_{\theta, 2}\right)}{Z_{b} \bar{r} \bar{b} L_{B}} \\
c_{C L}=0.816 \sqrt{\frac{2 \Delta p_{C L}}{\rho_{3}}} \\
\dot{m}_{C L}=\rho_{3} Z_{b} s_{C L} L_{B} c_{C L} \\
\omega_{C L}=\frac{2 \dot{m}_{C L} \Delta p_{C L}}{\dot{m} \rho_{2} w_{2}{ }^{2}}
\end{gathered}
$$

where $\bar{r}$ and $\bar{b}$ are the mean radius and mean blade height respectively, $s_{C L}$ the clearance length and $c_{C L}$ the velocity through the gap. 


\subsubsection{Recirculation losses}

When the relative diffusion is high enough, the risk of stall is getting more and more significant. Due to the intense pressure gradient in the radial direction, a part of the flow turns around, goes through the vaneless space and re-enters the impeller channel leading in a recirculation flow. This loss is directly related to the absolute exit flow angle $a_{3}$. This is why the flow at the outlet has to be the more tangential in order to obligate the flow to continue towards the diffuser. The limit of the outlet flow angle is defined by Oh et al. [76]:

$$
\begin{gathered}
\tan a_{3}^{\prime}>2 \tan \beta_{3} \\
\tan \alpha^{\prime}{ }_{3}=\frac{w_{\theta, 3}}{c_{r, 3}}
\end{gathered}
$$

The enthalpy losses because of recirculation (parasitic losses) are estimated as:

$$
I_{R C}=\left(\frac{D_{f}}{2}-1\right)\left(\frac{w_{\theta, 3}}{c_{r, 3}}-2 \tan \beta_{3}\right)
$$

\subsubsection{Disk friction}

The disk friction work input coefficient is calculated by the model of Daily and Nece [111] that gives the torque coefficient $\left(C_{M o}\right)$ as a function of the characteristic Reynolds number and the relative roughness of the disk.

$$
I_{D F}=0.75 C_{M} L_{i m p} \frac{1-\left(r_{2 t} / r_{3}\right)^{5}}{r_{3}-r_{2}} \frac{\rho_{3} U_{3} r_{3}{ }^{2}}{2 \dot{m}}
$$

\subsubsection{Leakage losses}

The gap between the tip and the shroud in the impellers allows a part of the $\mathrm{CO}_{2}$ to flow from the pressure side to suction side of the blade, resulting in pressure losses at this portion of the flow that is driven by the blades [106]. The difference between the tip clearance losses (internal loss) and the leakage losses (parasitic loss) is that the first one is generated because of the flow passing from the pressure side to suction side of the blade through the clearance gap, while the leakage is produced because the flow passes through the clearance gap from the impeller outlet back to the low-pressure regions of the overall impeller. The enthalpy losses because of leakage flow are estimated using the following expression:

$$
I_{L}=\frac{\dot{m}_{L} v_{L}}{2 U_{3} \dot{m}}
$$




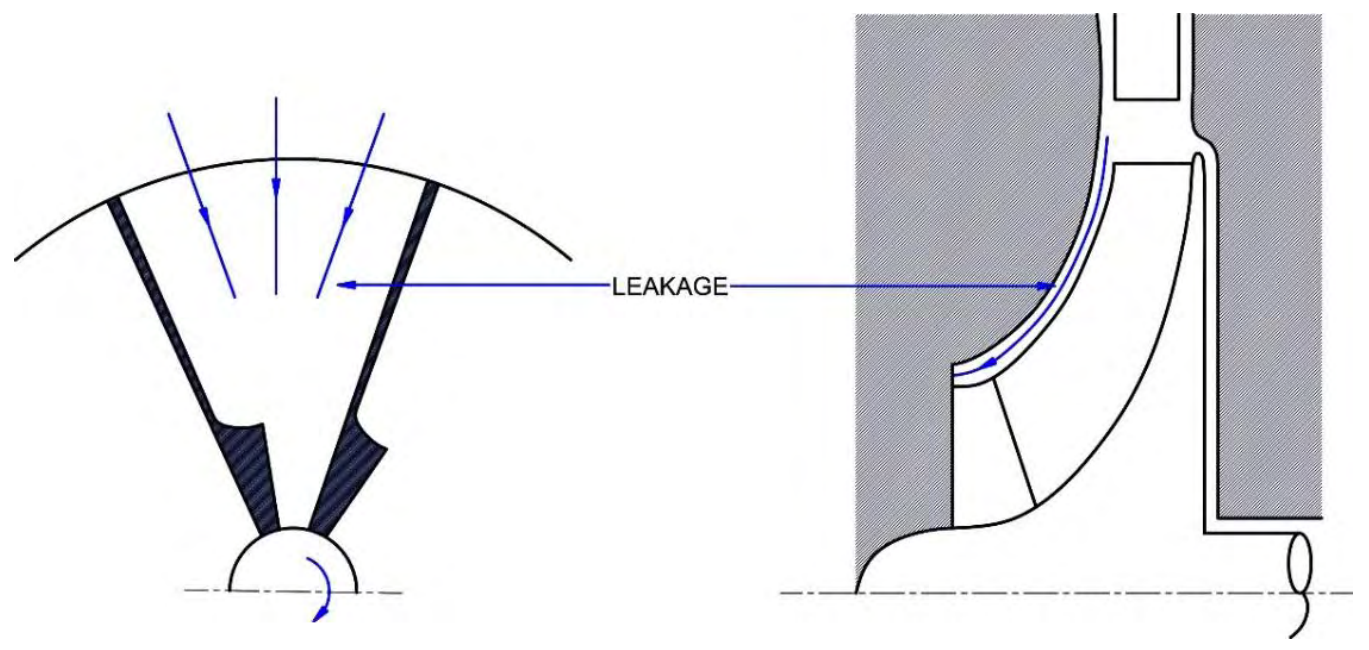

Figure 5.4. Leakage affecting clearance loss [100].

\subsubsection{Impeller performance}

The overall impeller loss coefficient is estimated as the sum of the coefficients of all the loss mechanisms developed above.

$$
\omega_{i m p}=\omega_{B L}+\omega_{H S}+\omega_{\lambda}+\omega_{m i x}+\omega_{f r}+\omega_{C L}
$$

Taking the output of the inducer as the input to the impeller model, the outlet pressure is estimated using the overall pressure losses and the relative reference of frame:

$$
P_{03, r}=P_{03, r, s}-f_{c}\left(P_{02, r}-P_{2}\right) \sum_{2 \rightarrow 3} \omega_{i}
$$

where $f_{c}$ is considered the correction factor and is employed to account for the fact that the losses are applied at the impeller outlet. The factor is defined as [79]:

$$
f_{c}=\frac{P_{03, r}}{P_{02, r}}
$$

Finally, the enthalpy parasitic losses are estimated as:

$$
\Delta h_{o, \text { parasitic }}=U_{2}^{2}\left(I_{R C}+I_{D F}+I_{L}\right)
$$

Then, the aerodynamic and the design efficiency were estimated as: 


$$
\begin{gathered}
\eta_{\text {aerodynamic }}=\frac{h_{o 3, i d}-h_{02}}{\Delta h_{o, \text { Euler }}} \\
\eta_{\text {design }}=\frac{h_{o 3, i d}-h_{02}}{\Delta h_{o, \text { Euler }}+\Delta h_{o, \text { parasitic }}}
\end{gathered}
$$

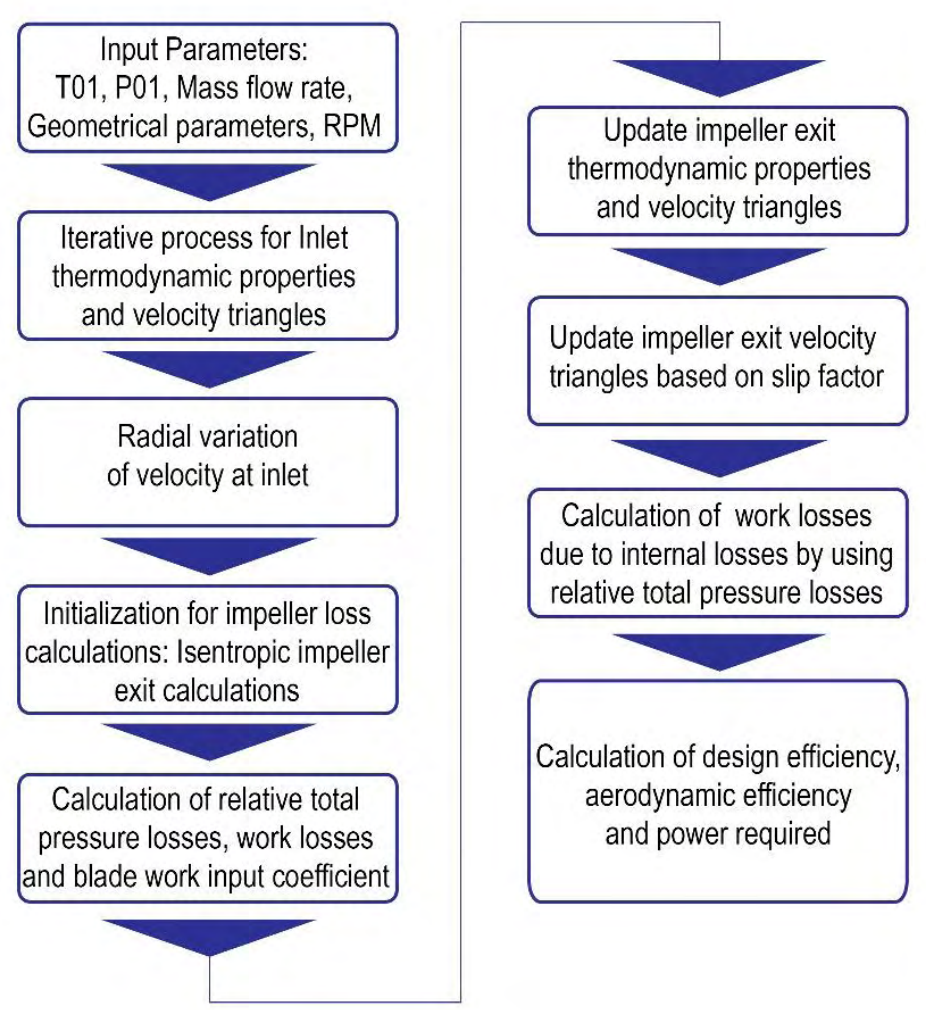

Figure 5.5. Algorithm of impeller mean line analysis.

\subsubsection{Vaneless space}

In centrifugal impellers, there is a vaneless space between the impeller and the vaned diffuser that is often referred to as the vaneless diffuser. The role of the vaneless diffuser is to diffuse the flow in low Mach numbers and insert it appropriately in the following vanes of the diffuser, avoiding shock waves at the leading edge of the vanes. In order to estimate the pressure loss coefficient, some loss mechanisms have to be taken into account. The wall friction loss due to the boundary layer leads to important blockage at the vane leading edge that has to be estimated, thus the vaneless space has to be minimized in order to reduce this blockage if it is appreciable. 


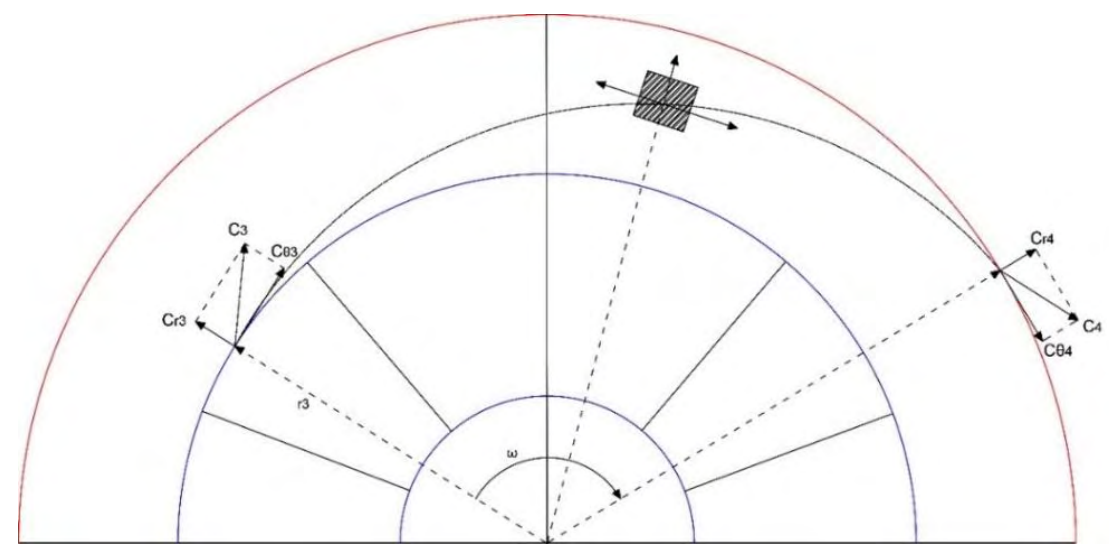

Figure 5.6. Flow distribution inside the vaneless diffuser.

Furthermore, the variation of the mean streamline angle produce total pressure losses, because of the motion presented in Figure 5.6. In order to calculate these coefficients, the geometry and the outlet velocity of the vaneless space has to be estimated. Aungier has proposed the following estimation method that is applied in this study [112]. The flow diffusion model is based on an analogy to classical two-dimensional diffusers. The diffuser is divided into an infinite number of diffusers of length $d m$ and area ratio established by the radius ratio.

$$
\begin{gathered}
\dot{m}=2 \pi r b \rho c_{r} \\
b C_{r} \frac{d\left(r c_{\theta}\right)}{d r}=-r c c_{\theta} c_{f} \\
\frac{1}{\rho} \frac{d p}{d m}=\frac{c_{\theta}^{2} \sin a_{C}}{r}-c_{r} \frac{d c_{r}}{d m}-\frac{c c_{r}}{b} c_{f}-\frac{d I_{D}}{d m}-I_{C} \\
h_{0}=h+\frac{c^{2}}{2}
\end{gathered}
$$

The total enthalpy remains constant during the process. If density is considered constant for the very small length of the diffuser, and applying Bernoulli's equation between $r$ and $r+d r$ where the velocity reduces $(-d c)$, the following area ratio is obtained:

$$
A_{R}=1-\frac{d c}{c}
$$

The divergence parameter to the annular case is defined by Johnston [113]: 


$$
\begin{gathered}
D=-\frac{b}{c} \frac{d c}{d m} \\
D_{m}=0.4(b / d m)^{0.35} \cos a
\end{gathered}
$$

The efficiency of the annular diffuser based on experimental information, is calculated as:

$$
E=\left\{\begin{array}{cc}
1, & \text { if } D \leq 0 \\
1-0.2\left(D / D_{m}\right)^{2}, & \text { if } 0 \leq D \leq D_{m} \\
0.8 \sqrt{D / D_{m}}, & \text { if } D \geq D_{m}
\end{array}\right.
$$

The diffusion loss term is given by:

$$
\frac{d I_{D}}{d m}=-2\left(P_{o}-P\right)(1-E) \frac{1}{\rho c} \frac{d c}{d m}
$$

When stall takes place:

$$
I_{D}=0.65\left(P_{o}-P\right)\left(1-\frac{(r b)_{m}}{r b}\right) \frac{1}{\rho}
$$

The local area without stall $(r b)_{m}$ is calculated as the outlet area of the equivalent diffuser angle of $9^{\circ}$.

$$
(r b)_{m}=(r b)_{3}\left(1+0.16 m / b_{3}\right)
$$

The curvature of the flow streamline results in total pressure losses that are described by [78]:

$$
I_{C}=\frac{\kappa_{m}\left(P_{o}-P\right) c_{m}}{13 \rho c}
$$

Where the curvature is defined as: $\kappa_{m}=-\partial a_{c} / \partial m$.

Furthermore, the blockage effect is modeled for the vaneless diffuser. The blockage factor is expressed as:

$$
B=\delta /(4 b)
$$

If the boundary layer at the diffuser inlet is known, $c_{\theta e}$ can be calculated. The angular momentum of the core flow, $r c_{\theta e}$, is easily calculated using the flow field at the inlet to the vaneless space and the blockage factor that permits closing the boundary layer model. 


$$
r c_{\theta}=\frac{r c_{\theta e}\left(1-\frac{2 \delta}{4.5 b}\right)}{1-B}
$$

The velocity $C_{\theta e}$ has to be conserved until the boundary layer fills the passage $\left(\delta=\frac{b}{2}\right)$.

\subsubsection{Vaned diffuser}

In centrifugal compressors, energy is transferred to the fluid by the impeller. Even though centrifugal impellers are designed for efficient diffusion within the blade passage, approximately half of the energy imparted to the fluid remains as kinetic energy at the impeller exit. The vaned diffuser in conjunction with the vaneless space, convert the remaining kinetic energy of the fluid into pressure energy.

The parameter E, was introduced by Aungier [78] in order to evaluate the offer of vaned diffuser in comparison with a vaneless, taking into account the radius ratio $\mathrm{R}$ at the outlet and inlet section and the area ratio of the vaned diffuser $A_{R}$.

$$
\begin{gathered}
E=\frac{R^{2}\left(A_{R}^{2}-1\right)}{A_{R}{ }^{2}\left(R^{2}-1\right)} \\
A_{R}=\frac{b_{5} r_{5} \sin \beta_{5}}{b_{4} r_{4} \sin \beta_{4}}
\end{gathered}
$$

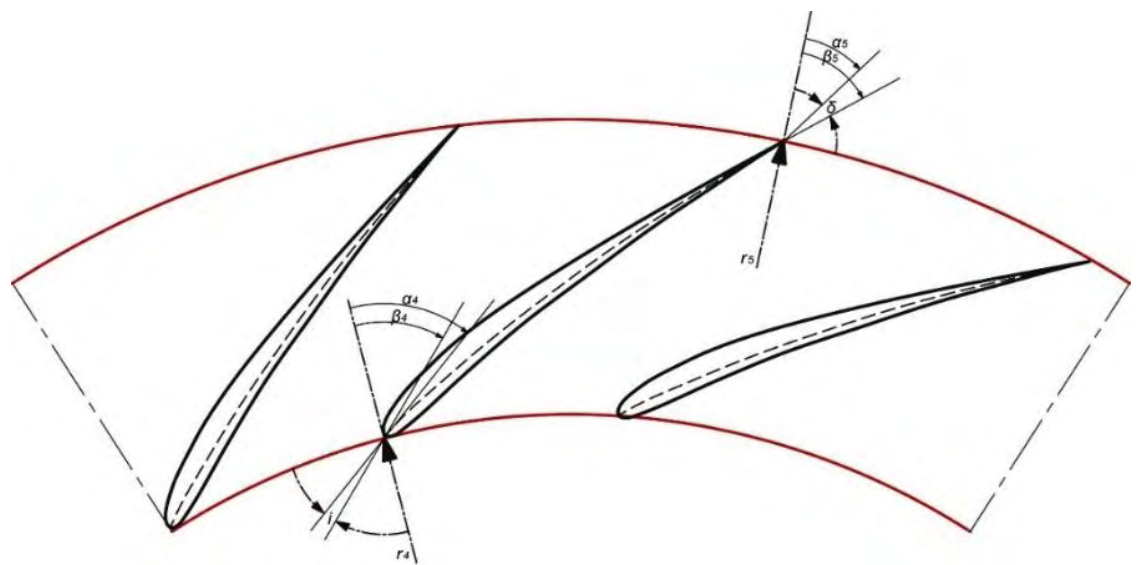

Figure 5.7. Geometry of vaned diffuser. 


\subsection{Inverse Problem}

The inverse problem consists the procedure of estimating the geometry of the centrifugal compressor for given operating conditions that have been derived by the thermodynamic analysis of the cycle. Since the solution of the direct problem in the previous section has led to a good accordance with the published data, the inverse problem will use the same set of equations in different way. For the direct problem the input was the inlet conditions and the geometry of the compressor, in order to estimate the outlet conditions, while for the inverse problem the input is the inlet and outlet conditions, in order to estimate the compressor geometry. The design procedure includes the preliminary design of the compressor for the given operating conditions, the model for the two-dimensional geometry and finally the development of the three-dimensional compressor model. The design procedure is presented in Figure 5.8.

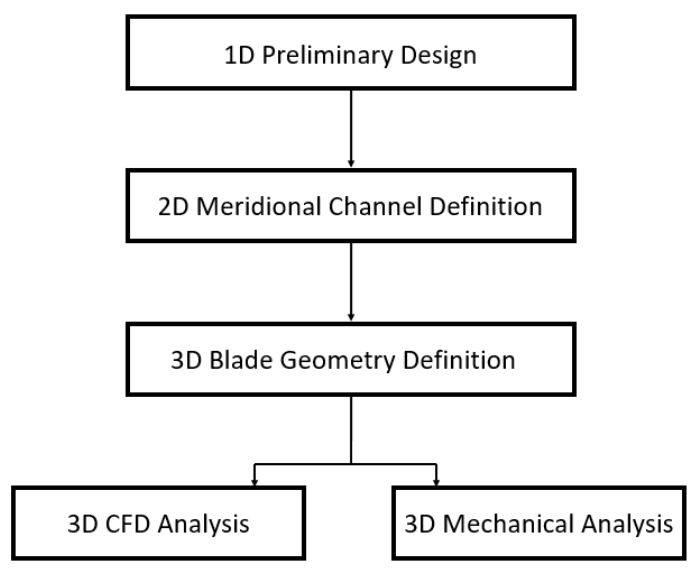

Figure 5.8. Centrifugal compressor design and analysis process.

\subsubsection{Preliminary design}

The preliminary design is based on a procedure developed by Balje in 1981 [72] for every type of turbomachine, based on the specific speed $\left(N_{S}\right)$ and the specific diameter $\left(D_{S}\right)$. Balje suggests that the performance of the compressor depends on the set of specific speed-diameter, when Reynolds number exceeds $2 \times 10^{5}$. So, given a couple of $N_{s}$ and $D_{s}$, the efficiency of each type of compressor can be defined. The Balje diagram (Figure 2.8) is considered a valid tool for the preliminary design of the centrifugal compressor, even if carbon dioxide is not a conventional fluid for turbomachinery. 


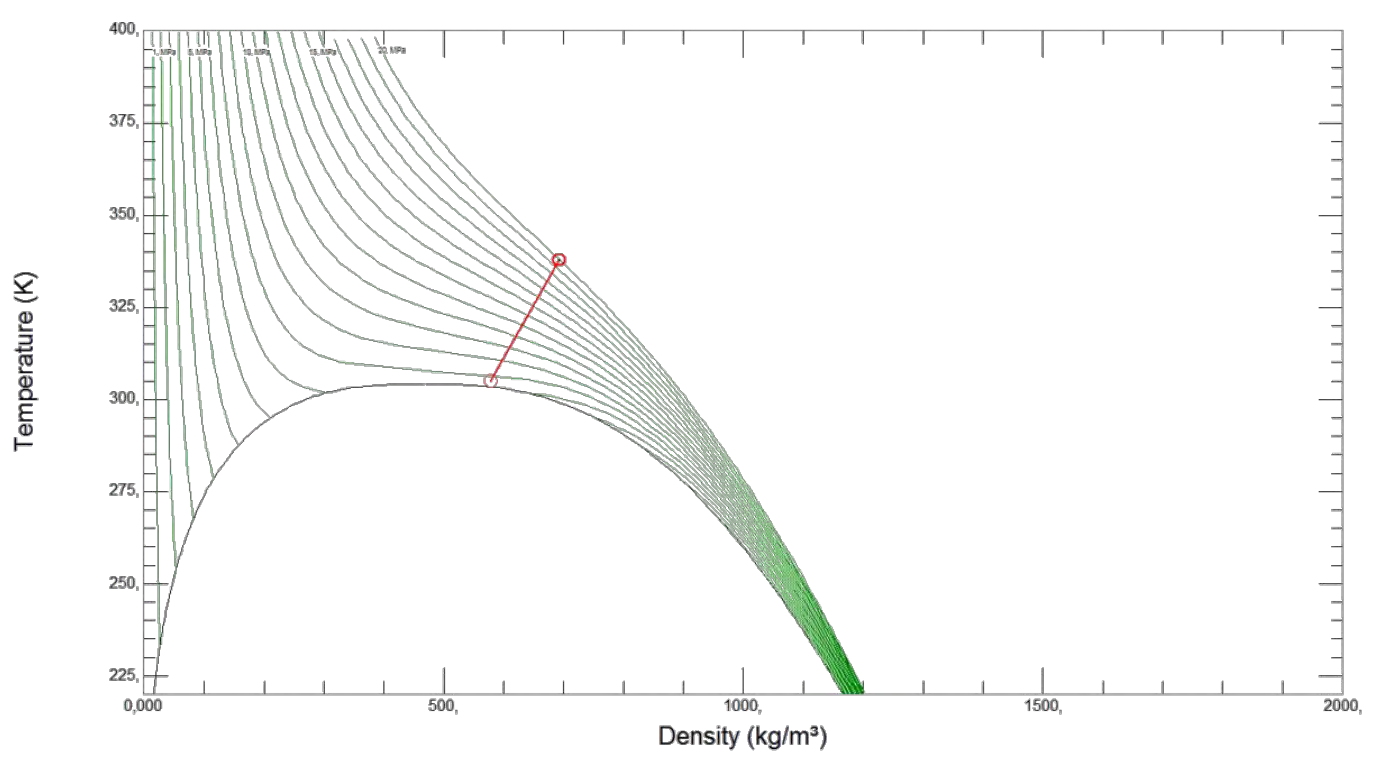

Figure 5.9. Density change during compression procedure in temperature-density diagram.

The specific speed and specific diameter are defined by the following expressions:

$$
\begin{gathered}
N_{s}=\frac{\left(\frac{\dot{m}}{\rho}\right)^{0.5} \Omega}{\left(\Delta h_{s}\right)^{0.75}} \\
D_{s}=\frac{d_{t}\left(\Delta h_{s}\right)^{0.25}}{\left(\frac{\dot{m}}{\rho}\right)^{0.5}}
\end{gathered}
$$

where $\dot{m}$ the mass flow rate $(\mathrm{kg} / \mathrm{s})$ is, $\rho$ is the density $\left(\mathrm{kg} / \mathrm{m}^{3}\right), \Omega$ is the shaft speed $(\mathrm{rad} / \mathrm{s}), \Delta h_{s}$ is the isentropic enthalpy rise $(\mathrm{J} / \mathrm{kgK})$ and $d_{t}$ is the tip diameter $(\mathrm{m})$ of the blade. The enthalpy rise corresponds to the work done for the compression presented in Figure 5.9.

The study considers a rotating speed of $3600 \mathrm{rpm}$ and a mass flow of 1908 $\mathrm{kg} / \mathrm{s}$. As mentioned in Chapter 2, centrifugal compressors are characterized by a specific speed of 0.4-1.8 with optimum values ranging at 0.6-0.77 [25]. The specific speed is selected to achieve the highest efficiency, and for given volumetric flow and enthalpy change, the specific diameter is estimated, resulting in a tip diameter of the centrifugal compressor. The specific speed was selected 0.65 and the specific diameter 4 , resulting in an isentropic efficiency $85 \%$. The specific speed was selected to be 0.65 and the specific diameter 4 , resulting in an isentropic efficiency of $85 \%$. The rotating speed of the compressor was selected $3600 \mathrm{rpm}$ in order to be turbine driven, without need of gearbox. 


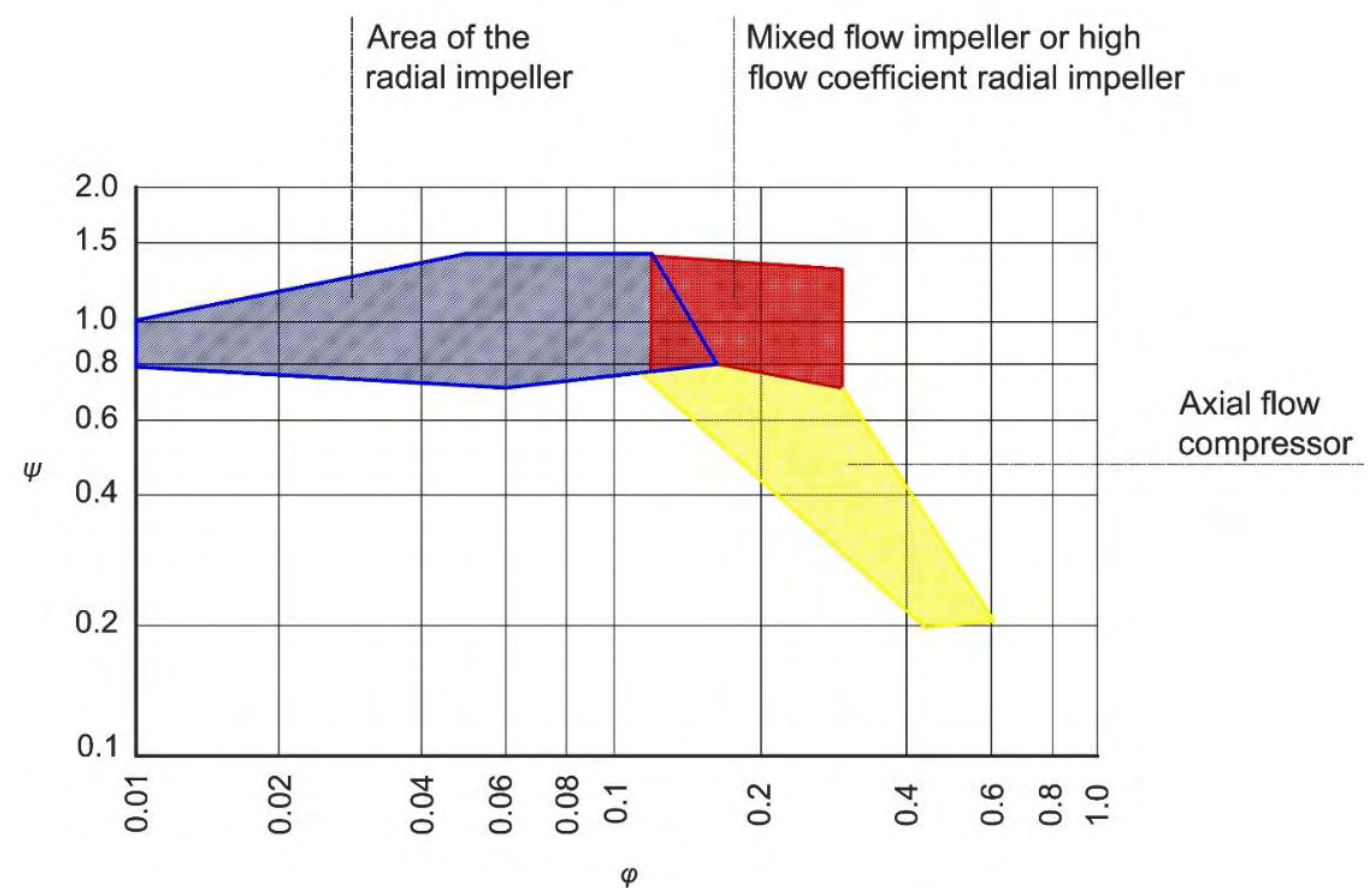

Figure 5.10. Typical values of flow and stage coefficients for radial and axial compressors.

Moreover, the flow and stage coefficients were estimated during preliminary design, in order to check if their values correspond to radial design according to the following diagram. The flow coefficient $\left(\Phi=C_{x} / U\right)$ was estimated at 0.031 and the stage coefficient $\left(\Psi=\Delta h_{\text {stage }} / U^{2}\right.$ ) at 0.59 , which correspond really close to the region of radial impeller according to the diagram of Figure 5.10.

\subsubsection{Compressor design procedure}

With the total pressure and temperature at the inlet, $P_{01}$ and $T_{01}$, which are inputs to the code, it is possible to obtain the stagnation enthalpy and the stagnation entropy through the following equations.

$$
\begin{aligned}
& h_{01}=f\left(P_{01}, T_{01}\right) \\
& s_{01}=f^{\prime}\left(P_{01}, T_{01}\right)
\end{aligned}
$$

Since the entropy between static and stagnation states remains the same, it is feasible to obtain the thermophysical properties directly using the static enthalpy and the stagnation entropy at that same point. The absolute velocity at the inlet is a known variable, which is constant throughout the entire span of the blade. 


$$
\begin{gathered}
h_{1}=h_{01}-\frac{c_{1}{ }^{2}}{2} \\
s_{1}=s_{01} \\
P_{1}=f\left(h_{1}, s_{1}\right) \\
T_{1}=f^{\prime}\left(h_{1}, s_{1}\right) \\
\rho_{1}=f^{\prime \prime}\left(h_{1}, s_{1}\right) \\
c_{P 1}=f^{\prime \prime \prime}\left(h_{1}, s_{1}\right)
\end{gathered}
$$

At the outlet, in order to obtain the thermophysical properties, the stagnation properties in this stage need to be addressed first. Then, the same procedure is repeated for the impeller inlet.

The pressure ratio is an input to the compressor design model, so the stagnation enthalpy at the outlet can be obtained through the equation:

$$
h_{03}=h_{02}-\frac{h_{03 s}-h_{02}}{\eta_{t t}}
$$

Where $h_{03 s}$ is the enthalpy that the flow would have at the outlet if the compression process was isentropic. As the entropy at the inlet is already known and the total pressure at the outlet is determined through the pressure ratio and its inlet counterpart:

$$
h_{03}=f\left(P_{03}, s_{2}\right)
$$

Therefore, the efficiency needs to be guessed and the model will be iterating until a convergence is achieved with the results of the performance analysis via loss models. Consequently, assuming identical total and static entropy again, all the outlet static thermophysical properties can be obtained, using the procedure described above.

The following steps are taken in order to complete the design procedure for the inducer and impeller:

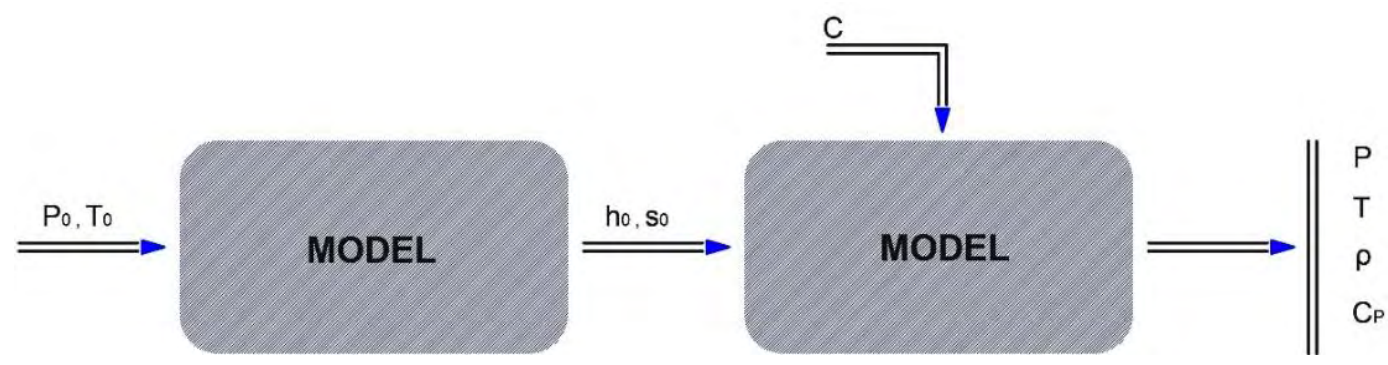

Figure 5.11. Estimation procedure for static variables for the compressor inducer. 
1. A hub to tip ratio is selected and a shroud inlet radius is guessed.

2. The velocity diagram at the inlet and the geometry are derived assuming null incidence $\left(\beta_{2}=\alpha_{2}\right)$

3. The static conditions are checked in order to ensure supercritical flow.

4. The impeller outlet radius is derived by preliminary design.

5. It is assumed that the meridional velocity at inlet and outlet are identical.

6. The total-to-total efficiency $\left(\eta_{t t}\right)$ and the total pressure loss coefficient $\left(L_{C}\right)$ of the system apart from impeller [79], are guessed in order to meet the desired conditions at the impeller exit.

$$
L_{C}=\frac{P_{03}-P_{05}}{P_{03}}
$$

7. The total pressure at impeller's exit $P_{03}$ is calculated as:

$$
P_{03}=\frac{P_{01} P R_{\text {stage }}}{1-L_{C}}
$$

8. The slip factor, distortion factor and loss coefficients for impeller are guessed.

9. The velocity diagram at the impeller exit is estimated.

10. The impeller blade exit angle is estimated:

$$
\beta_{3}=\operatorname{atan}\left[\frac{\left(\frac{V_{\theta 3}}{\sigma}-U_{3}\right)}{\lambda V_{m 3}}\right]
$$

11. The static conditions at the impeller exit are calculated.

12. The geometry of the impeller is derived based on the assumptions. Then the direct model presented in the previous section is used in order to estimate slip, distortion factor and the pressure loss coefficients.

13. The whole procedure is repeated until the convergence between the assumptions and the results for step 12.

Having completed the previous steps, the design of the inducer and the impeller is fixed, and the procedure continues to vaneless space and diffuser.

The significant parameters for the vaneless space design are the radius, the blade height and the inlet blade angle. For the blade height the assumption that $h_{b 4} \leq$ $h_{b 3}$ is used. Monge [79] presents the equation:

$$
\frac{r_{4}}{r_{3}}=1+\frac{90-a_{4}}{360}+\frac{M_{3}^{2}}{15}
$$


Assuming a small diffusion of the flow, the meridional velocity at the inlet of the diffuser is guessed and for a radius ratio between 1.06 and 1.12 [78], the inlet flow angle of the diffuser $\left(a_{4}\right)$ can be estimated.

Finally, for the design of the vaned diffuser some significant parameters presented in the previous section have to be defined according to Aungier [78]. The design for the vaned diffuser comprised the acceptance of ranges presented in Monge [79]:

- Divergence angle lower than $11^{\circ}$.

- Loading parameter lower than $1 / 3$.

- A design parameter: $1.5 \leq E \leq 1.7$

The following diagram presents the design procedure flowchart.

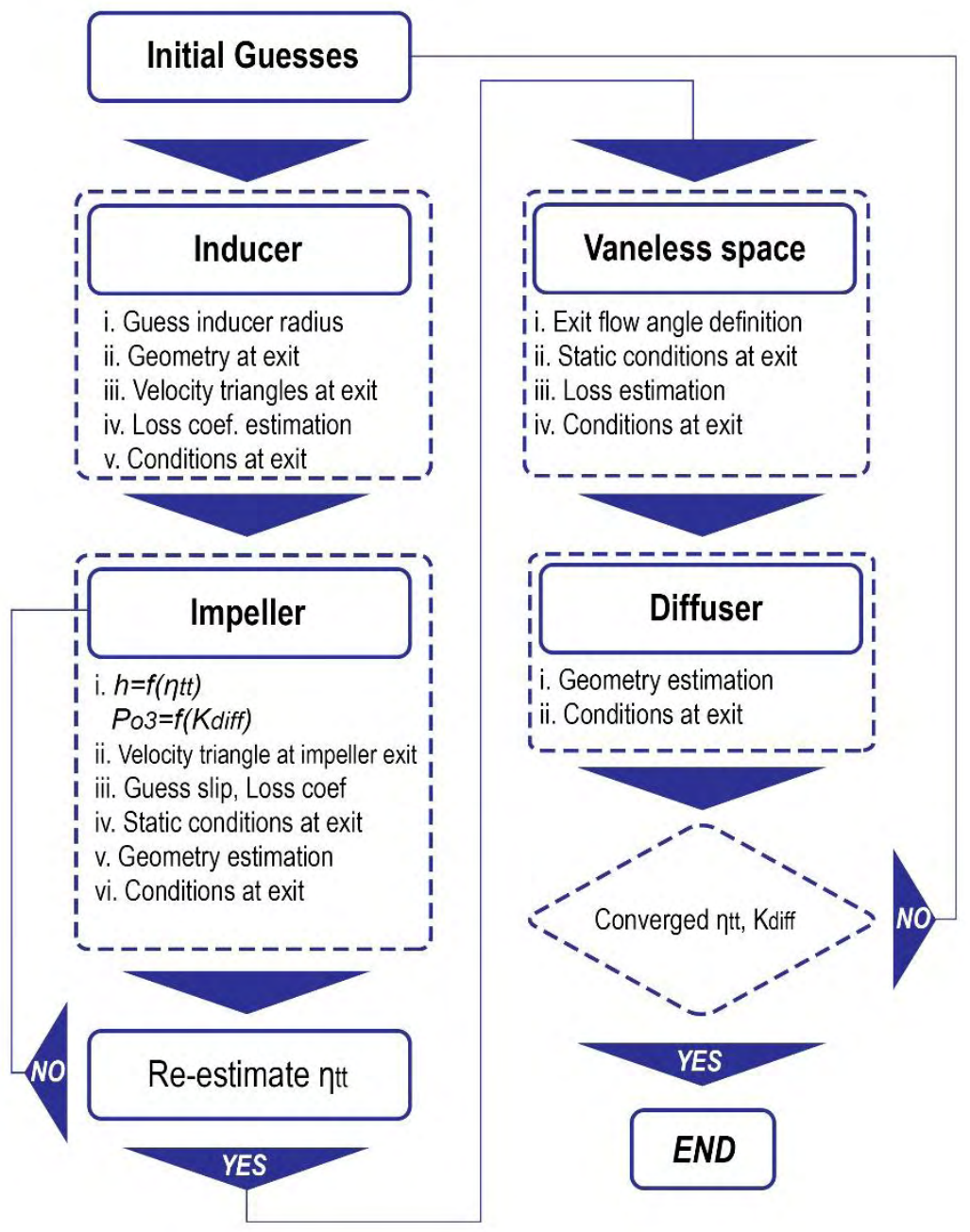

Figure 5.12. Flowchart for inverse problem solution. 


\subsection{Numerical Method}

The investigation of the flow phenomena depicting the centrifugal compressor performance, require the solution of a set of non-linear partial differential equations. The set of equations for compressible fluid are applied for the compressor flow in three dimensions and RANS equations are employed.

The differences between the flow in the heat exchanger and the compressor are firstly the rotating flow in the impeller that has to be predicted, and secondly the interface definition between the rotating and stationary compressor parts. Moreover, the flow in a centrifugal compressor is highly turbulent, presenting Reynolds numbers in the range of $10^{5}-10^{7}$, leading in the development of turbulent boundary layers at the blades' walls. Therefore, the turbulence model used in the compressor study is the shear stress transport (SST), which prevents the overprediction of the turbulence viscosity.

The CFD analysis requires a connection between the rotating and stationary domains. There is an interface connecting the two domains, so when a change occurs in one domain it would be transferred to the other. For this study, the critical interfaces are these connecting the impeller with the stationary inlet and the diffuser. There are different methods that handle this interface, such as frozen rotor and stage/mixing plane. For this analysis, the frozen rotor method is used. The frozen rotor model, which is also known as Multiple Reference Frame (MRF) model, treats the flow from one component to the next by changing the frame of reference across the interface, while keeping the relative orientation of the components fixed. The position of the mesh elements does not change between the two interfaces, but is fixed for the whole computational domain. The frozen rotor model produces a steady-state solution at each time instant, taking into consideration the two frames interaction, but the phenomena varying with time are neglected.

\subsubsection{Numerical procedure}

The software used for the study of centrifugal compressor is Ansys CFX, as it is suitable to model turbomachinery flows and is designed to predict rotating flow sufficiently. The entire process of the CFD analysis includes five general steps: 3D geometry definition (designed with BladeGen), meshing (Turbogrid from Ansys suite is used), CFX-pre where the carbon dioxide properties are introduced and the boundary conditions are selected, CFX-solver and CFD-Post, where postprocessing tools are available.

Ansys CFX solver follows the computational grid generation with finite volume method, which is employed to solve the conservation equations for mass, momentum and energy for each one, as was described in Chapter 4.4.6. The description of the general steps is described in the following sections. 


\subsubsection{Geometry and Mesh generation}

Since the geometry design is derived from the one-dimensional analysis, the three-dimensional geometry of the impeller and diffuser was created using Ansys BladeGen, a tool that allows to create and modify appropriately axial and radial turbomachinery. Two different geometry models were created, one for the impeller geometry and another for the diffuser geometry, and were combined later for the whole compressor flow simulation. Figure 5.13 and Figure 5.14 present the compressor three-dimensional model, which can be adapted according to the user's input data.
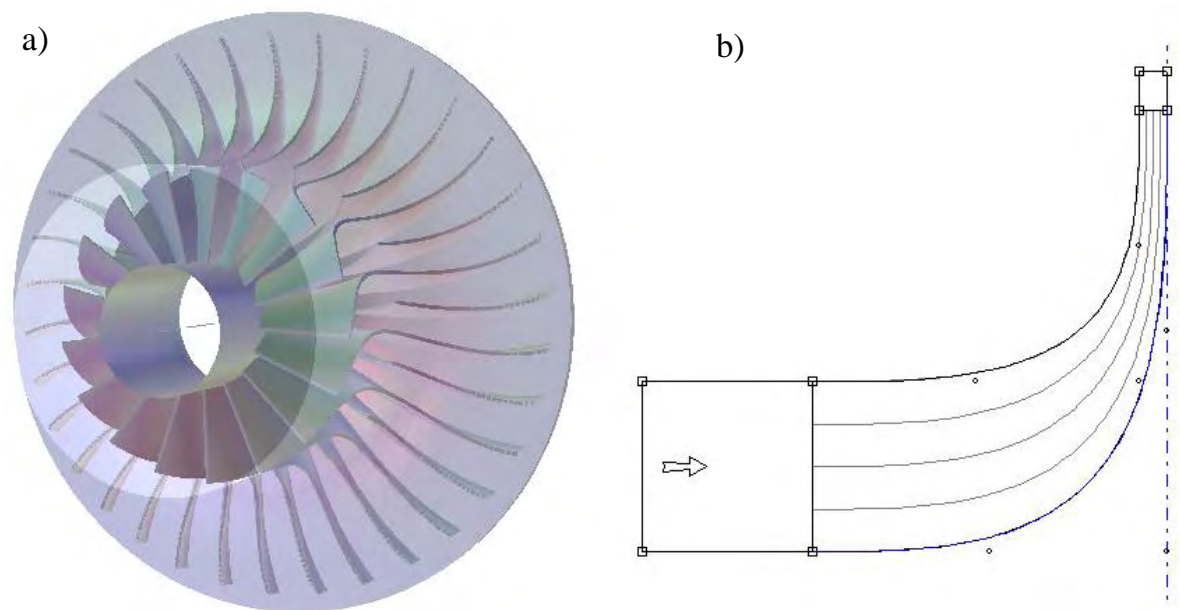

Figure 5.13. Impeller geometry derived by inverse problem: a) full geometry, b) section of blade.

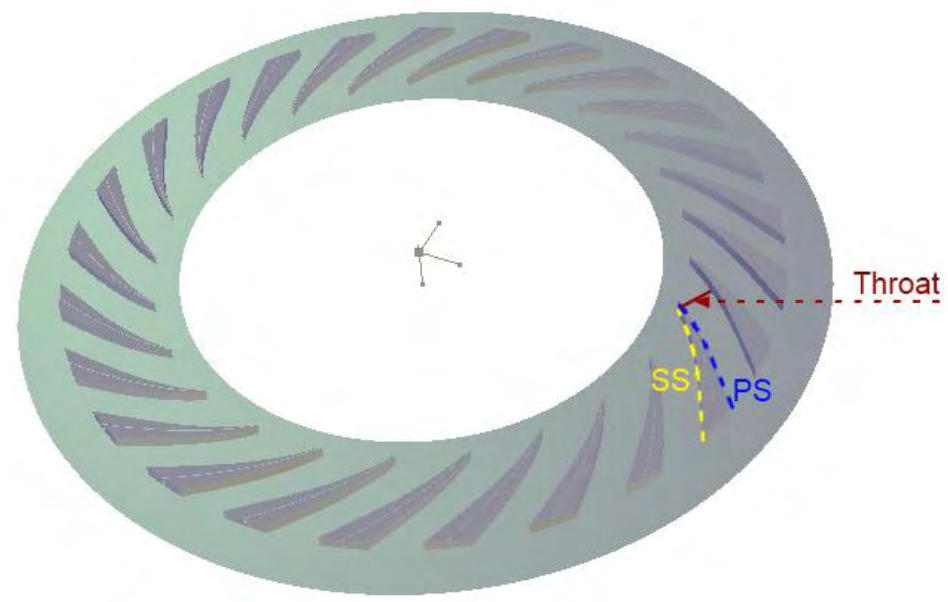

Figure 5.14. Vaned diffuser geometry. 
The mesh generation was carried out with TurboGrid, a mesh-generation tool included in Ansys Workbench and designed specifically for turbomachinery. This meshing tool has a large degree of autonomy, which leads to a less time-consuming meshing. When used in conjunction with the Ansys embedded software for boundary conditions definition, the software automatically detects the type of turbomachinery being imported and generates the mesh in a time efficient manner.

The high performance of this meshing tool is mainly attributed to its topology. The grid is divided into different structured blocks that act as a framework for the positioning of the mesh. The blocks are positioned by default to achieve the desired grid conditions. Each topology block handles the details of the topology surrounding a blade. Each block contains a specific number of mesh elements. The mesh elements vary in size across each of the topology blocks to achieve a smooth transition between blocks. The smooth transition is achieved by node biasing, which also helps reduce the number of mesh elements. The most common topology method employed is ATM Optimized with an automatic method.

The Figure 5.15 and Figure 5.16 show the meshes that were used for the aerodynamic analysis of the centrifugal compressor, for the impeller and diffuser respectively.

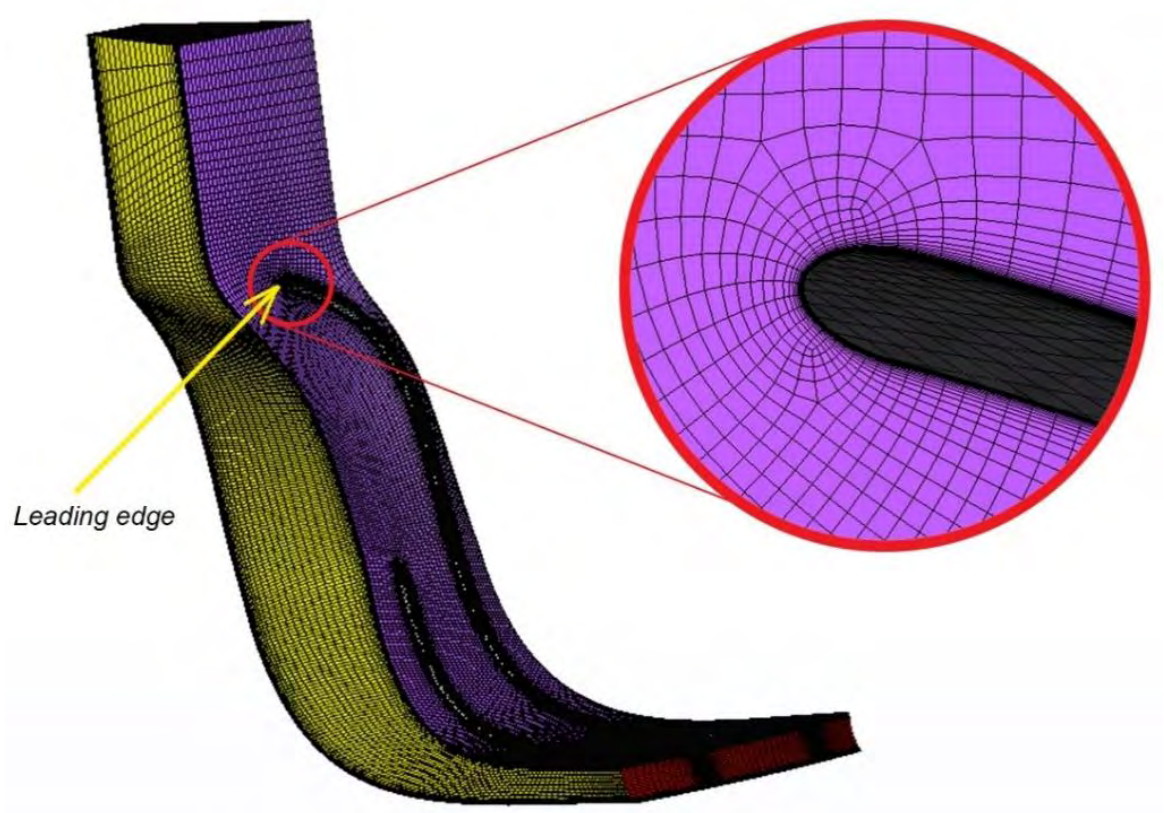

Figure 5.15. Mesh of the blade and impeller passage. 


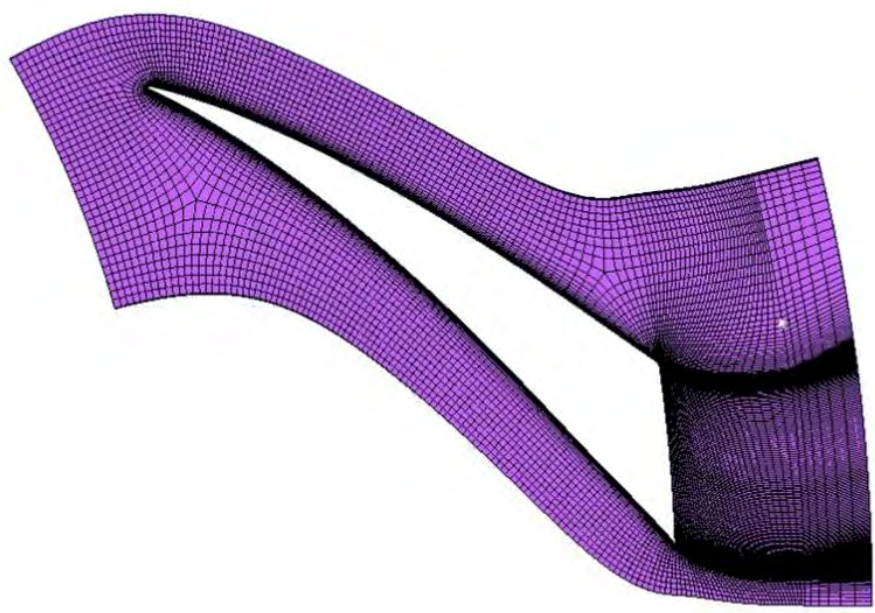

Figure 5.16. Mesh around the diffuser.

\subsubsection{Mesh independency study}

Mesh sensitivity analysis is necessary to ensure the independence of the results on the mesh size and nodes positions. Various meshes were investigated in order to find out their impact on the solution quality. As presented in Table 5.1 the pressure, temperature, density and Mach number present a very small relative difference between the thicker and the coarser mesh. The second mesh-case with a total number of nodes that equals $2.3 \times 10^{6}$ was the one adopted for the simulations. It has to be noted that the tip clearance gap increases significantly with the number of nodes for the flow simulation.

Table 5.1. Outlet thermodynamic properties variation with the mesh refinement.

\begin{tabular}{lccccc}
\hline Mesh & $\mathbf{1}$ & $\mathbf{2}$ & $\mathbf{3}$ & $\mathbf{4}$ & $\mathbf{5}$ \\
\hline Total Nodes & $2.2 \times 10^{6}$ & $2.3 \times 10^{6}$ & $3.6 \times 10^{6}$ & $4 \times 10^{6}$ & $6.2 \times 10^{6}$ \\
Pressure $(\mathrm{MPa})$ & 18.27 & 18.28 & 18.285 & 18.3 & 18.31 \\
Temperature $(\mathrm{K})$ & 343.03 & 343.15 & 343.18 & 343.24 & 343.27 \\
Density $\left(\mathrm{kg} / \mathrm{m}^{3}\right)$ & 493.04 & 493.023 & 493.07 & 493.14 & 493.16 \\
Mach & 0.50 & 0.505 & 0.506 & 0.506 & 0.508 \\
\hline
\end{tabular}

\subsubsection{Boundary conditions}

The simulation is set up by implementing the Turbo mode. This mode is used for turbomachinery and it automatically creates the boundary conditions and interfaces of the component. The Turbo mode is made to complement TurboGrid. The location of the boundary conditions and interfaces is illustrated in Figure 5.17. 
The basic settings of the system were set to a reference pressure of $0 \mathrm{~Pa}$, and a rotational speed of $3600 \mathrm{rpm}$. A total energy heat transfer model was selected as well as the inclusion of viscous work. As mentioned before, the Shear Stress Transport (SST) turbulence model was selected along with the high speed (compressible) wall heat transfer model. The inlet boundary conditions were defined in terms of the total inlet pressure and temperature. The inlet flow was defined to be fully axial, without swirl. Furthermore, the inlet boundary condition was defined as stationary, and the initial turbulence intensity was chosen to be $5 \%$. The outlet boundary condition was defined in terms of the outlet mass flow with an active mass flow outlet constraint and the boundary is set to stationary. For the entire investigation a smooth wall was assumed. The boundary conditions corresponding to the moving part of the impeller were set as rotating, while the parts such as the diffuser, extended inlet, and shroud were specified as a counter rotating wall. Interfaces were set up within the tip clearance region. Periodicity was employed to simplify the modelling of the component, providing the benefit of having to model just one passage. Moreover, periodicity allowed the modelling of turbomachinery to be simplified, and rotational periodic interfaces were set up on the outer sides of the passage.

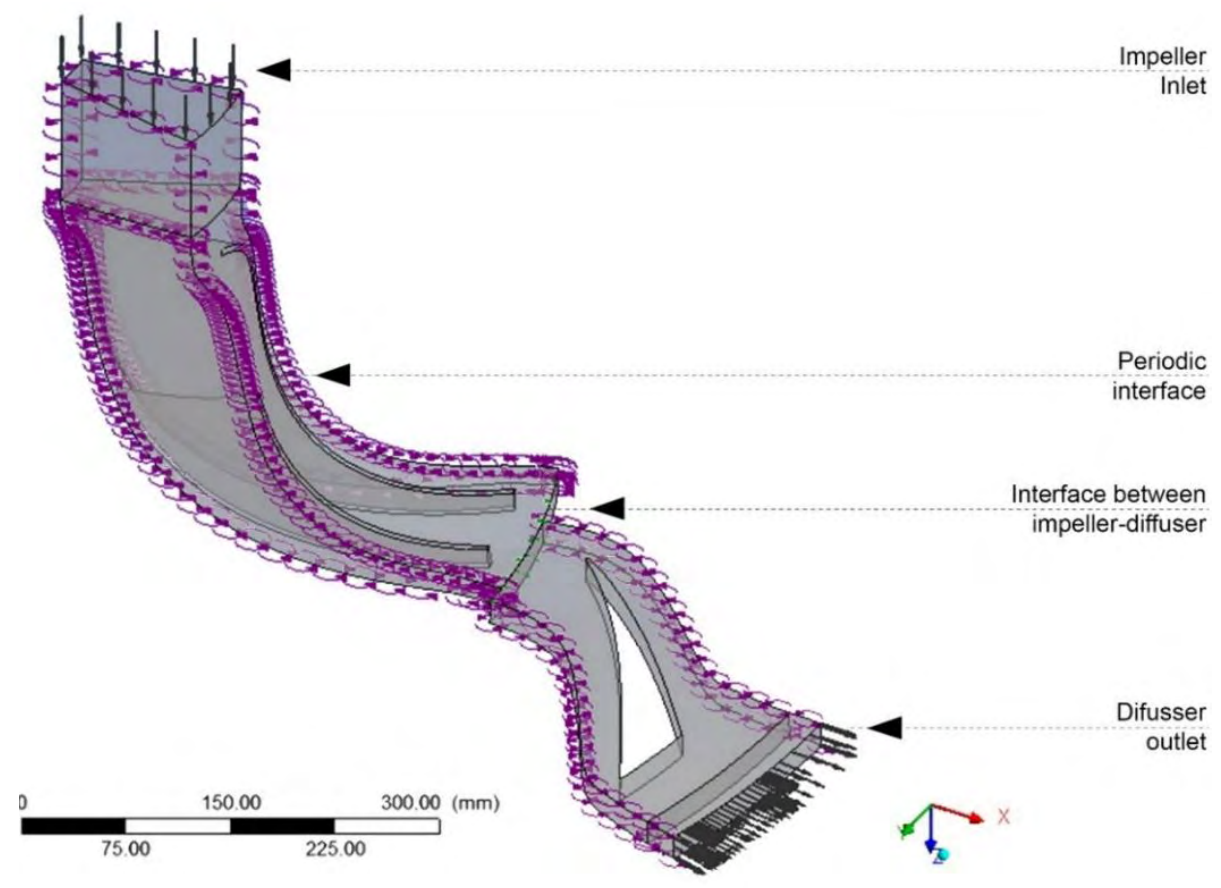

Figure 5.17. Location of the inlet and outlet periodic conditions and periodic interface. 


\subsubsection{Real gas effects on numerical solution}

The computational fluid dynamics analysis needs a library databased with the thermodynamic properties of the carbon dioxide, in order to use them in the solution of the continuity, momentum and energy equilibriums. However, Ansys CFX does not include real gas properties of $\mathrm{CO}_{2}$ in its libraries. Therefore, a real gas properties (RGP) file, which is an external look-up table including the thermodynamic properties of $\mathrm{CO}_{2}$ was developed and was coupled with the flow solver. The real gas properties (RGP) table includes nine main properties: specific entropy, specific enthalpy, speed of sound, specific volume, specific heat at constant pressure and volume, dynamic viscosity, thermal conductivity, and partial derivative of pressure with respect to specific volume at constant temperature. For the look-up table generation, an in-house MATLAB code was generated, using the fluid properties provided by NIST Refprop database, employing the Span and Wagner model as discussed in Chapter 3. The RGP table generated had a range from 290 to $400 \mathrm{~K}$ and 5 to $25 \mathrm{MPa}$ for temperature and pressure respectively, in order to cover all the operational range of the compressor and prevent the extrapolating of the solver in any stage of the simulation.

Moreover, in order model accurately the behavior of $\mathrm{CO}_{2}$ in the pseudo-critical region and overcome instabilities due to the highly nonlinear behavior in the simulation, the RGP table was chosen to have more values around the critical point. As the solver interpolates the properties between table points, it is obvious that the number of values approaching the critical point can affect the solution accuracy.

To acquire a solution with sufficient accuracy the RGP table with the desired number of parameters was investigated. It was selected to examine various grids in the pseudo-critical region, increasing the number of values in this region, from 100 points up to 600 points and investigating the differences of the specific heat capacity. Figure 5.18 presents the accuracy of the table at constant pressure 7.69 $\mathrm{MPa}$ that consists the inlet and Figure 5.19 presents the accuracy of table for pressures $10 \mathrm{MPa}$ and $15 \mathrm{MPa}$, as the fluid properties are far from the critical point.

It can be observed that for pressures 10 and $15 \mathrm{MPa}$, the table accuracy is really high (around $0.004 \%$ ), even for 100 points and there is no significant change between 500 and 600 points. This occurs because carbon dioxide approaches the behavior of perfect gas far at these pressures. The region that presents the most significant instabilities and the simulation of its condition is difficult to predict in the inlet of the compressor at 7.69 MPa. For this pressure, various points were investigated, resulting in a good accuracy for 500 points, as the relative error is lower than $0.3 \%$ and the difference with 600 points is not significant. Less points around the critical point, such as 300 or 400 presented a lower accuracy, leading in an inaccurate prediction of the flow behavior. 


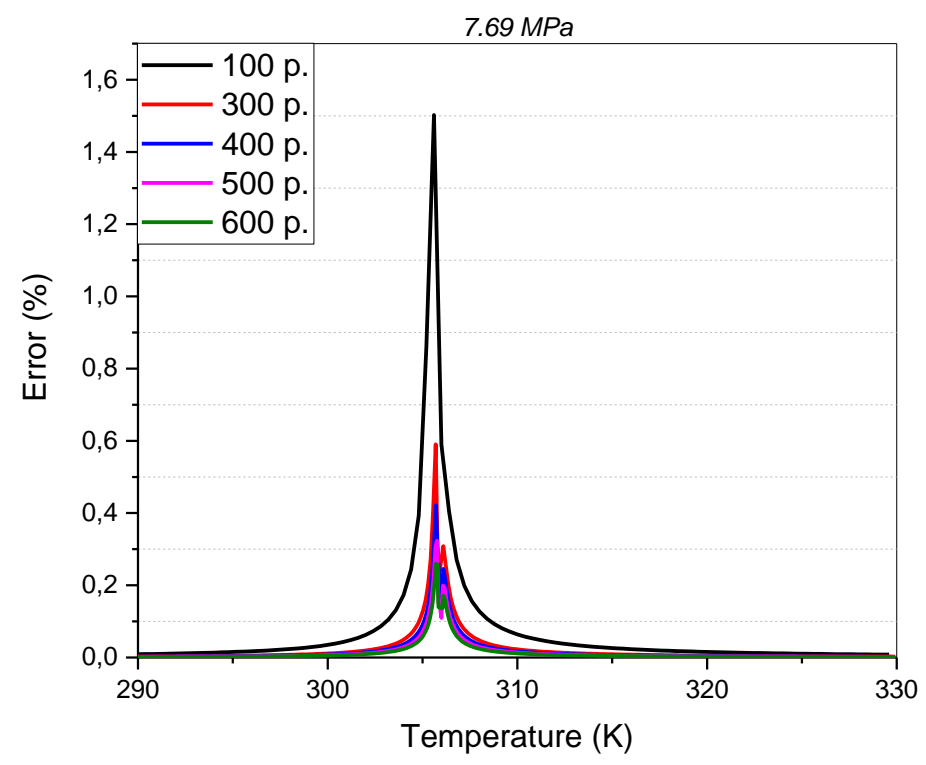

Figure 5.18. Accuracy of specific heat in the pseudocritical region for pressure 7.69 MPa.
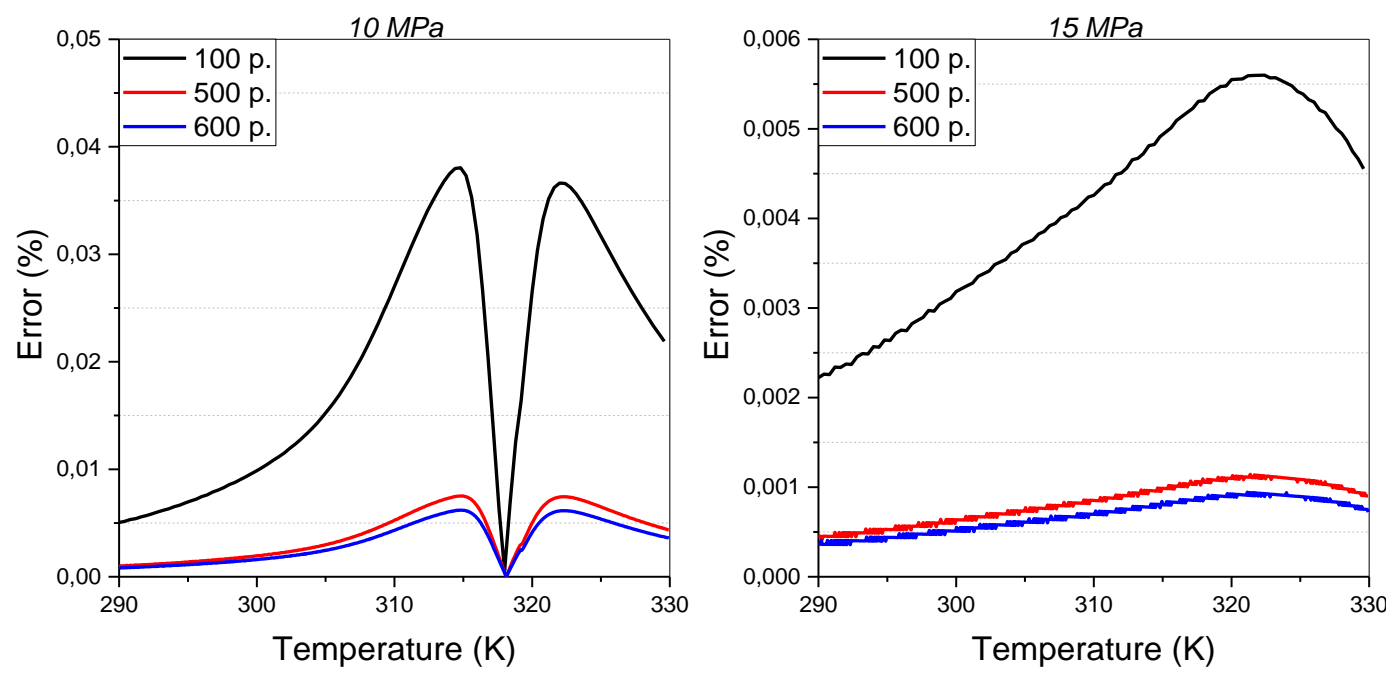

Figure 5.19. Accuracy of specific heat in the pseudocritical region for pressure 10 and $15 \mathrm{MPa}$.

\subsubsection{Possibility of condensation}

At the compressor inlet, at the suction side of the impeller leading edge, there is possibility of condensation as the flow properties may cross the saturation line because of the flow acceleration. The fluid starts condensing when the process 
leads the fluid to attain the saturation line and the residence time in the nucleation region is significantly larger than the residence time. The condensation occurs under non-equilibrium conditions and the droplet growth depends on the magnitude of the divergence from the equilibrium state.

The non-equilibrium state has to do with the consecutive situations of the fluid during an expansion. During an expansion process of a superheated fluid, at a specific instant, the fluid reaches the saturation curve. When the expansion continues under equilibrium conditions, droplets start to form at the saturation curve and the expansion continues along the saturation curve. In this case, there is no entropy generation during condensation as the process follows successive equilibrium states.

Although, various experiments have showed that nucleation does not start at the saturation curve and maybe no condensation occurs unless the expansion continues. Since the expansion process continues further than the saturation curve, the fluid enters a metastable region (non-equilibrium state), which is represented by the temperature difference relative to the corresponding equilibrium state. This temperature difference is defined as supercooling degree and is calculated using the saturation temperature $\left(T_{S}\right)$ at vapor pressure (the value that corresponds to the saturation curve) and the temperature of the vapor $\left(\Delta T=T_{S}\left(p_{v}\right)-T_{v}\right)$ or by the ratio of the fluid pressure to the equilibrium pressure $\left(s=p_{v} / p_{s}\right)$, defined as supersaturation.

When this temperature difference or the supersaturation attains a specific value, droplets start to form and the fluid is moving towards saturation curve and reaches a stable state, which is at lower pressure and temperature in comparison with the corresponding equilibrium condensation [114]. Moreover, the remaining time of the fluid in the metastable region affects the droplet generation and growth. The metastable properties have not been measured experimentally, so their estimation requires a numerical procedure. Span and Wagner model is solid only for the stable region, but in the metastable phase gives unreasonable results. Therefore, a real gas properties (RGP) table has to be defined for $\mathrm{CO}_{2}$, extrapolating the properties in the liquid domain in order to calculate the fluid pressure in a region close to the saturation curve. Having the fluid properties, the saturation pressure has been taken into consideration too, in order to estimate the supersaturation degree. An in-house MATLAB code was used to calculate the $\mathrm{s}-\mathrm{CO}_{2}$ properties in the specific region, in order to provide Ansys CFX solver with the appropriate $\mathrm{CO}_{2}$ properties. For this study a simple criterion was applied, regarding the value of supersaturation, in order to assess the possibility of condensation. The residence time of fluid in the unstable region was not estimated for this study. 


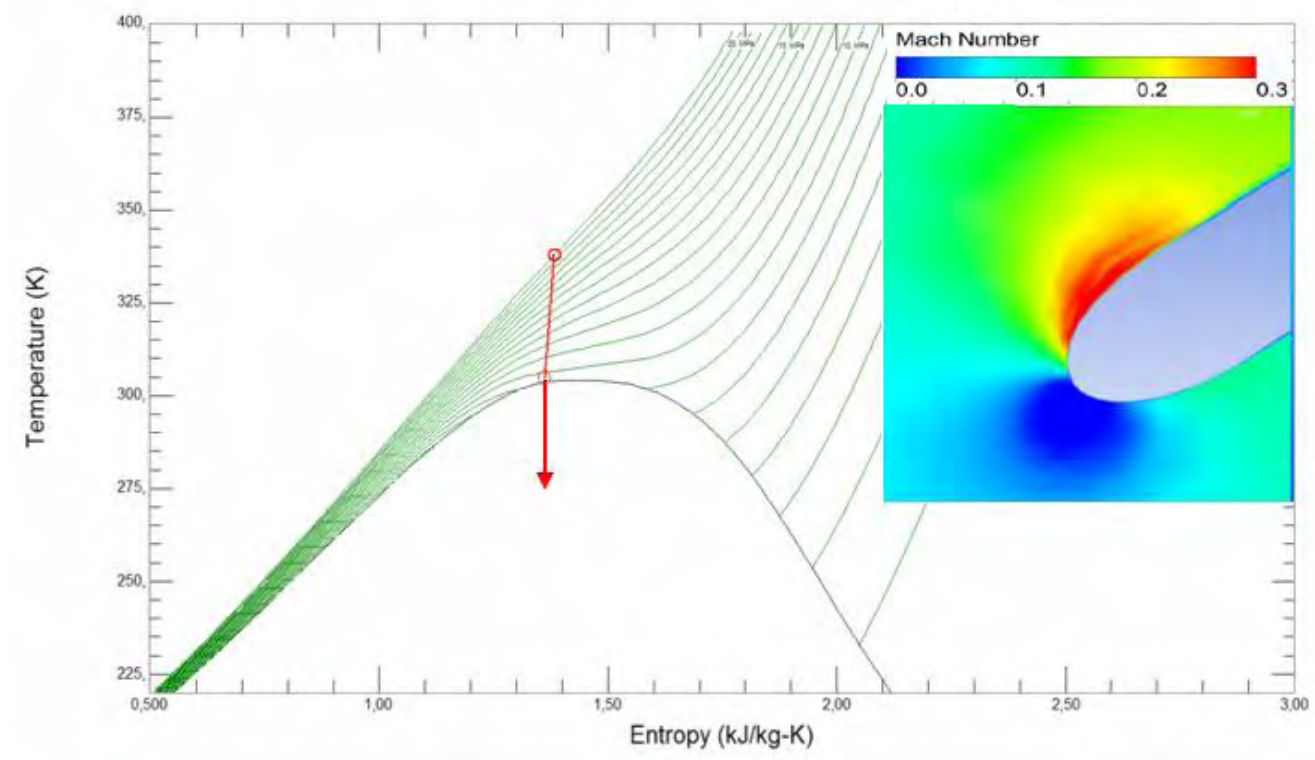

Figure 5.20. Drop inside the two-phase region because of flow acceleration.

\subsection{Conclusions}

This chapter presents the methods used for the centrifugal compressor design and analysis for this thesis, incorporating loss models. The 1-D design analysis was based on the approach of a direct problem estimating the pressure loss coefficients for each of the compressor's elements. Then the inverse problem was presented, leading in the compressor design that meets the thermodynamic cycle conditions, using the same methodology developed for the direct problem, but in a way that the thermodynamic properties are known and the geometry is derived. The governing equations for the compressible flow in three-dimensions have been presented in a previous chapter, so only the interface models and the numerical procedure for the compressor are presented, as the Reynolds-Averaged NavierStokes and turbulence model analysis is the same. The numerical procedure that was applied, including the geometry generation and the meshing of the derived centrifugal compressor was presented, as well as how the real gas effect influences the numerical solution. Moreover, the simulation of the $\mathrm{CO}_{2}$ behavior required a look-up table, which was created and employed by the flow solver, leading in a resolution of 500 points around the critical point presenting a relative error of $0.3 \%$ for the specific heat capacity estimation. For pressures far from the critical point, it was observed that the look-up table does not require high resolution as the relative error is around $0.003 \%$ even for 100 points around the critical temperature. Finally, a method to assess the possibility of condensation at the impeller inlet is 
discussed, taking into consideration the properties in the metastable region close to the saturation curve and a simple criterion regarding the deviation from the saturation condition. 


\section{Chapter 6}

\section{Printed-Circuit Heat Exchanger Performance}

This chapter presents the results of the above mentioned methods (presented in Chapter 4) regarding the modeling of printed-circuit heat exchangers. The results of the one-dimensional segmental analysis regarding the thermal-hydraulic performance are discussed, while numerical results of the PCHE simulation are presented and discussed. Finally, experimental cases for steady-state and dynamic performance of the heat exchangers are presented, leading in a final design geometry of the high-temperature recuperator of the considered recompression s$\mathrm{CO}_{2}$ Brayton cycle.

\subsection{One-Dimensional Model}

The mass flow rate of the carbon dioxide is distributed among the channels uniformly, so that the total amount of heat is divided in each sub-heater and the properties of carbon dioxide can be computed in each segment of the heat exchanger. The number of channels per cross-section area are adjusted to modulate the Reynolds number and ensure the pressure drop remains in the operational range that the cycle design parameters define (Table 6.1).

The Figure 6.1 shows Reynolds number in each control volume of both recuperators, which is above the transition range and the flow is considered fully turbulent. This behavior of the curve is due to the large variation in density of the fluid near a phase change, approaching the critical point. In the HTR the hot and cold sides have the same mass flowrate, and this is why Reynolds number curves are much closer for this recuperator in comparison with LTR.

Table 6.1. Specifications of Printed-Circuit Heat Exchangers.

\begin{tabular}{lcc}
\hline Parameter & HTR & LTR \\
\hline Number of channels & 11165 & 10160 \\
Channel diameter (mm) & 2.2 & 2.2 \\
Plate thickness (mm) & 0.5 & 0.5 \\
Channel pitch (mm) & 2.4 & 2.4 \\
\hline
\end{tabular}


Concerning the low temperature recuperator, the mass flow in the cold side of the recuperator is about the $60 \%$ of the mass flow in the hot side, resulting in a lower Reynolds number. Moreover, Reynolds number increase results in a decrease of the friction factor, affecting the Nusselt number.

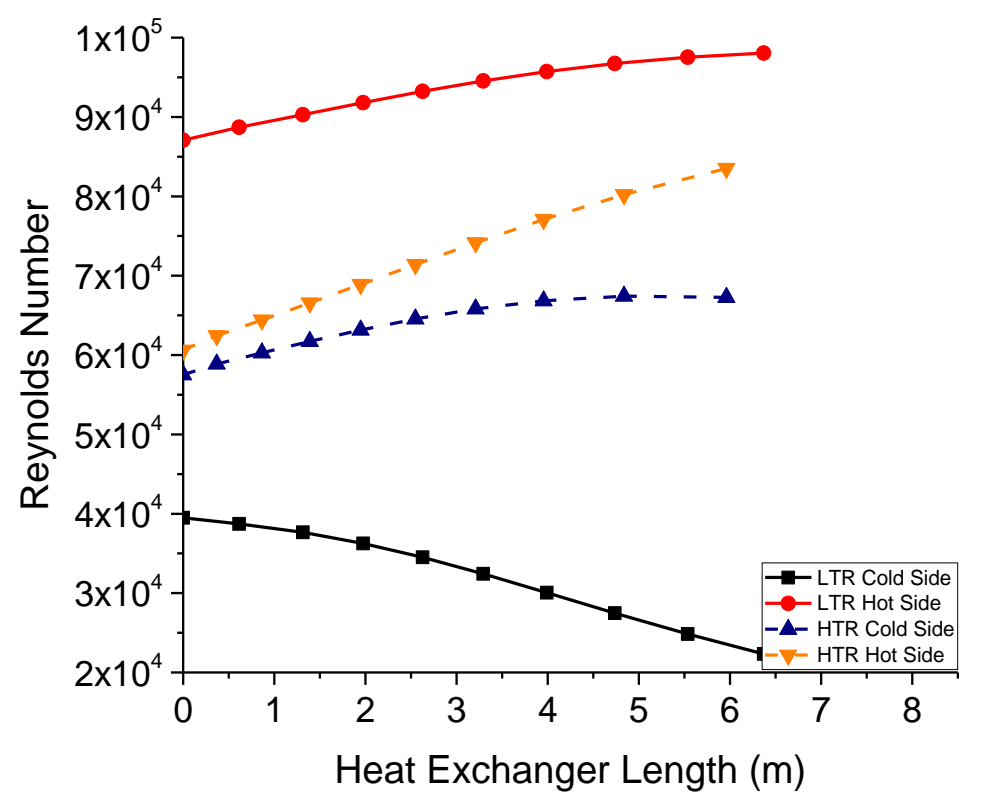

Figure 6.1. Reynolds number as a function of heat exchanger length.

The Nusselt number variation along the heat exchangers is presented in Figure 6.2, demonstrating the significant dependence of the Nusselt number on Reynolds number for turbulent flow compared to friction factor and Prandtl number. In the developed code Prandtl number decreases in the hot side as in every next sub-heater the temperature is getting higher.

The Nusselt number diagram presents the same behavior with this of Reynolds number diagram, indicating a large deviation between the hot and cold side of LTR and a small difference between hot and cold side of HTR. These two graphs show that Reynolds number is the main parameter that contributes to fluid heat transfer.

Figure 6.3 shows an interesting trend because the calculations are factoring in the variation of the fluid thermal conductivity as the fluids cool down and get closer to the critical point. It is observed that the HTR has a greater heat transfer rate along the heat exchanger, as the heat transferred in this recuperator is much larger and so does the overall heat transfer coefficient. On the other hand, at the beginning the NTU for LTR decreases and then seems to be more even as the LTR operates at lower temperatures of the fluid flow. 


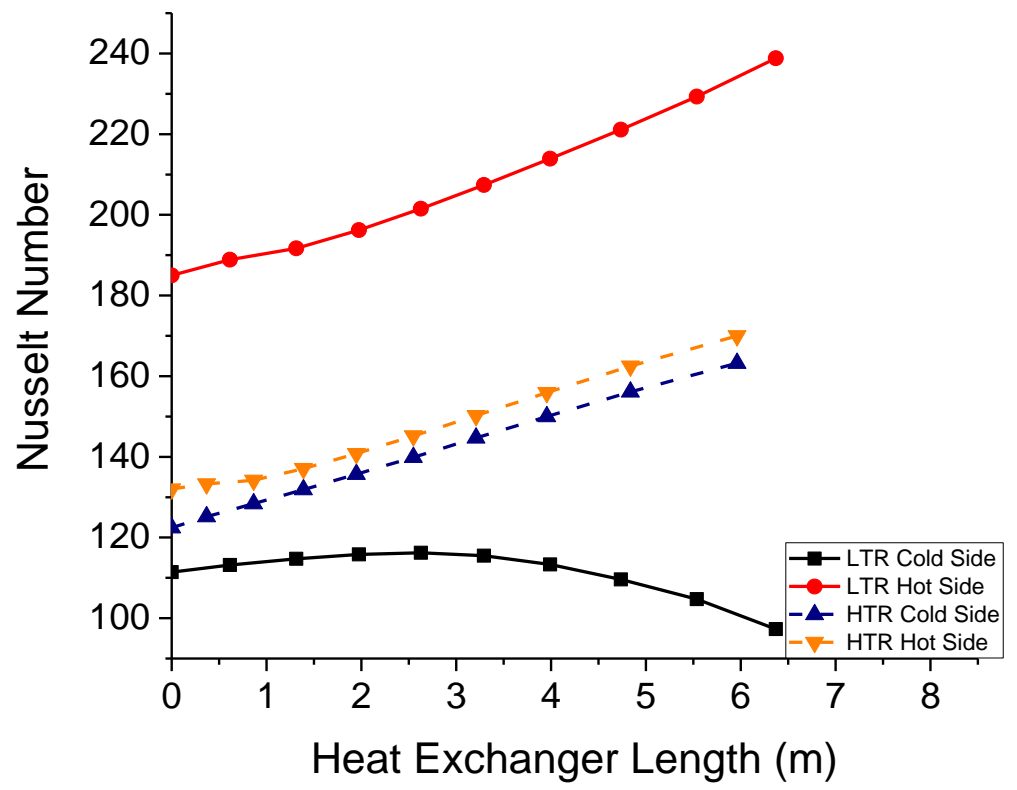

Figure 6.2. Nusselt number as a function of heat exchanger length.

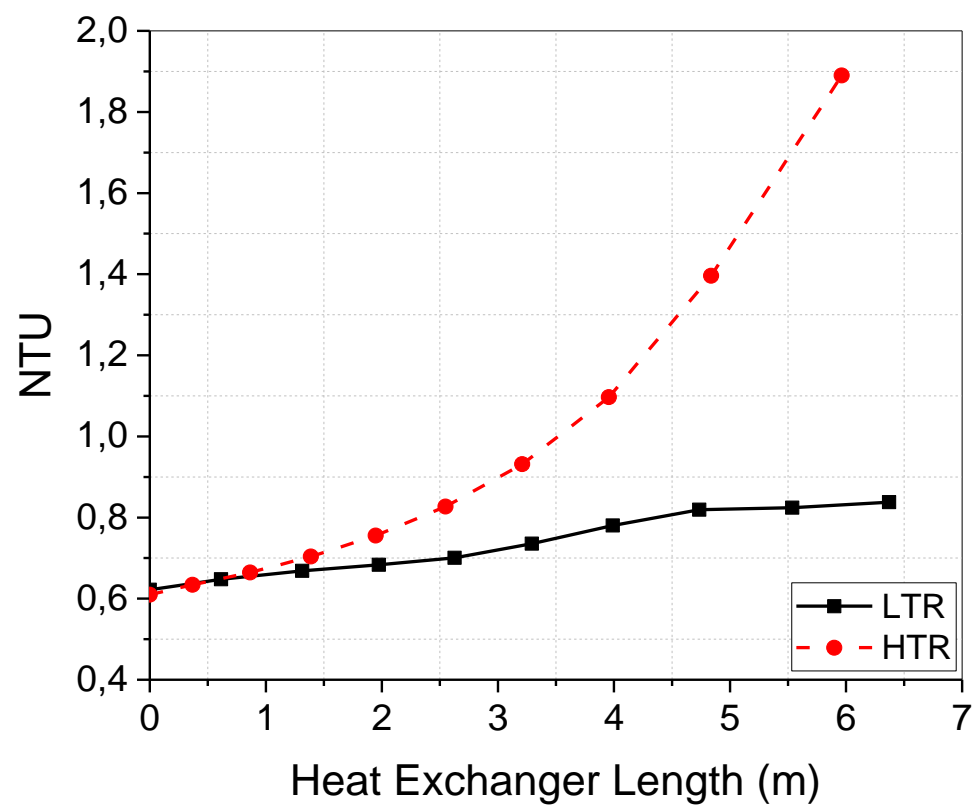

Figure 6.3. NTU diagram for high \& low temperature recuperators. 


\subsection{Experimental Tests}

\subsubsection{Experimental measurements}

After completing the instrumentation of the PCHE test rig, a number of experimental cases were carried out aiming to characterize the heat exchanger performance. The experimental procedure followed a prescribed pattern: First, the fan was switched in and air was circulated in the heat exchanger for several minutes. Carbon dioxide was allowed to flow in the heat exchanger after this initial period, in order to avoid high thermal stresses for the heat exchanger material. The test matrix is shown in Table 6.2. The s- $\mathrm{CO}_{2}$ mass flow varied across a range between 0.1 and $0.62 \mathrm{~kg} / \mathrm{s}$ and the operating pressure was increased from 7.6 to 11.5 MPa, while the maximum heat exchanger thermal duty during the tests was about $45 \mathrm{~kW}$.

For practical reasons, air is employed as the cooling medium during the laboratory tests, whereas in the real application at the air side we would have exhaust gas for waste heat recovery. This does not limit the applicability of the results, since the $\mathrm{CO}_{2}$ side behavior is the most interesting with this heat exchanger.

For each case, which represents an operating point, the heat transfer coefficients and the number of transfer units (NTU) were estimated. The NTU for each case was computed and incorporated to a typical capacity / NTU design chart for a counter-flow heat exchanger as it is presented in the heat exchanger literature. The coordinate axes of Figure 6.4 represent the dimensionless temperature changes $\mathrm{P}_{1}$ and $\mathrm{P}_{2}$ of the two streams, while $\mathrm{R}_{1}$ and $\mathrm{R}_{2}$ represent the dimensionless heat capacity rates.

Table 6.2. Test cases of the printed-circuit heat exchanger operation.

\begin{tabular}{|c|c|c|c|c|c|c|c|c|c|}
\hline & & \multicolumn{2}{|c|}{ Case 1} & \multicolumn{2}{|c|}{ Case 2} & \multicolumn{2}{|c|}{ Case 3} & \multicolumn{2}{|c|}{ Case 4} \\
\hline $\mathrm{CO}_{2}$ Conditions & & In & Out & In & Out & In & Out & In & Out \\
\hline Mass flow rate & $\mathrm{kg} / \mathrm{s}$ & \multicolumn{2}{|c|}{0.10} & \multicolumn{2}{|c|}{0.16} & \multicolumn{2}{|c|}{0.47} & \multicolumn{2}{|c|}{0.62} \\
\hline Temperature & K & 346.75 & 325.65 & 339.45 & 320.15 & 363.05 & 339.65 & 350.35 & 332.85 \\
\hline Pressure & $\mathrm{MPa}$ & 8.37 & 8.31 & 7.92 & 7.85 & 9.02 & 8.47 & 9.34 & 9.2 \\
\hline Specific heat cap. & $\mathrm{J} / \mathrm{kgK}$ & 1666 & 2519 & 1721 & 2689 & 1523 & 1906 & 1889 & 2510 \\
\hline Enthalpy & $\mathrm{kJ} / \mathrm{kg}$ & 477.72 & 436.66 & 470.63 & 431.67 & 497.13 & 462.09 & 467.4 & 438.69 \\
\hline Air Conditions & & In & Out & In & Out & In & Out & In & Out \\
\hline Mass flow rate & $\mathrm{kg} / \mathrm{s}$ & \multicolumn{2}{|c|}{0.26} & \multicolumn{2}{|c|}{0.39} & \multicolumn{2}{|c|}{0.58} & \multicolumn{2}{|c|}{0.97} \\
\hline Temperature & K & 324.6 & 341.24 & 319.2 & 335.43 & 322.9 & 351.21 & 322.07 & 340.15 \\
\hline Pressure & $\mathrm{kPa}$ & 113 & 111.93 & 113 & 112 & 113 & 110.2 & 113 & 110.14 \\
\hline
\end{tabular}




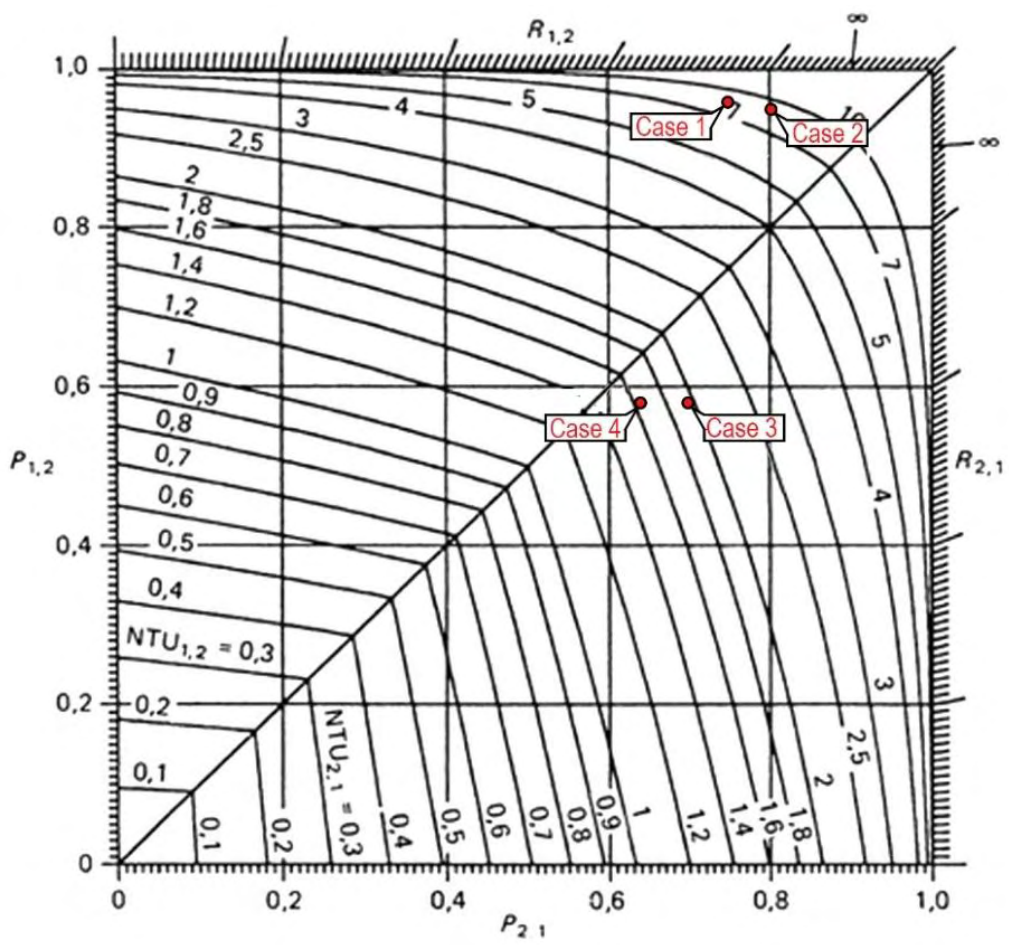

Figure 6.4. Position of the four heat exchanger operating points on the general dimensionless diagram of counter-flow heat exchangers' performance [115].

The number of transfer units is represented by the set of curves that are plotted in the chart [115]. It can be observed that the Case 1 and Case 2 from Table 6.2 present the highest number of transfer units compared to the NTU predicted for Case 3 and 4. The higher number of transfer units leads to higher heat transfer rate for Case 1 and 2, resulting to a higher effectiveness of the heat exchanger operating for these cases. The heat transfer rate, represented by number of transfer units (NTU), is crucial for effectiveness calculation and leads to high effectiveness of the heat exchanger. The low mass flows that present high number of transfer units, present also high effectiveness, up to $95 \%$.

Figure 6.5 shows the energy balance between the hot side $\left(\mathrm{S}-\mathrm{CO}_{2}\right)$ and the cold side (air) for the four cases presented in Table 6.2. The heat transfer calculation was based on the enthalpy change at the inlet and outlet of the heat exchanger, as the specific heat capacity cannot be considered constant for the $\mathrm{CO}_{2}$ side. It was found that the heat transfer rate to the cold side is slightly different from that of the hot side, presumably due to heat transfer from the outside shell of the MPCHE (which is not completely adiabatic). However, the energy balance always matches within $\mathrm{a} \pm 2 \%$ band, which confirms the successful thermal insulation of the system from the surroundings. Moreover, the estimated heat transfer includes the error of the temperature measurement uncertainty, as presented in the graph. 


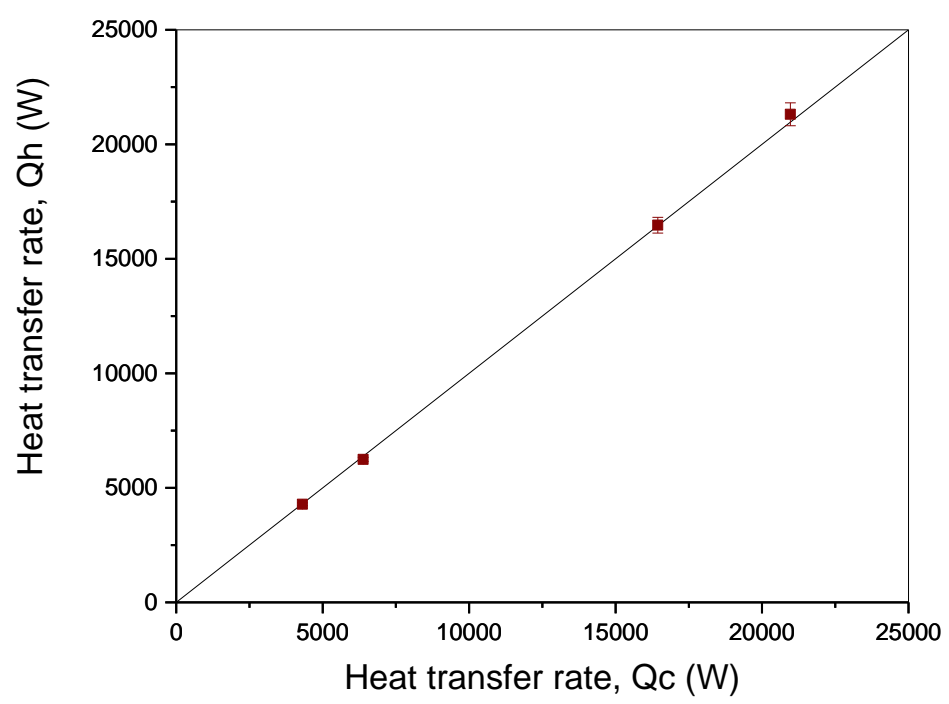

Figure 6.5. Energy balance between hot $\left(\mathrm{s}-\mathrm{CO}_{2}\right)$ and cold (air) side of the heat exchanger.

\subsubsection{Steady-state model}

A numerical one-dimensional model has been developed, in order to predict the printed-circuit heat exchanger behavior under varying operating conditions. The presented experimental cases support the model, which investigates the thermalhydraulic performance of the tested printed-circuit heat exchanger for an operational range.

The thermal performance of the MPCHE was investigated with the previously presented mathematical model. First of all, the possibility of the existence of pinchpoint was examined calculating the temperature distribution for every case. Figure 6.6 presents the result for Case 1 and Case 2, but similar behavior was observed across the entire range of the cases examined (Table 6.2). The results indicate that the heat exchanger operates without pinch-point. Pinch-point problem is highly dependent on heat exchanger geometry, and the minimum temperature difference proves the efficient design of the MPCHE.

Moreover, the Reynolds and Nusselt number were investigated because of their decisive impact on thermal-hydraulic performance of heat exchanger, as the heat transfer coefficient and the pressure drop depend on these dimensionless parameters. To investigate these flow characteristics, basic geometry of the heat exchanger was assumed, such as the number of channels, channel diameter and pitch. The assumptions were based on the outer dimensions of the tested heat exchanger and typical values for the internal geometry. 

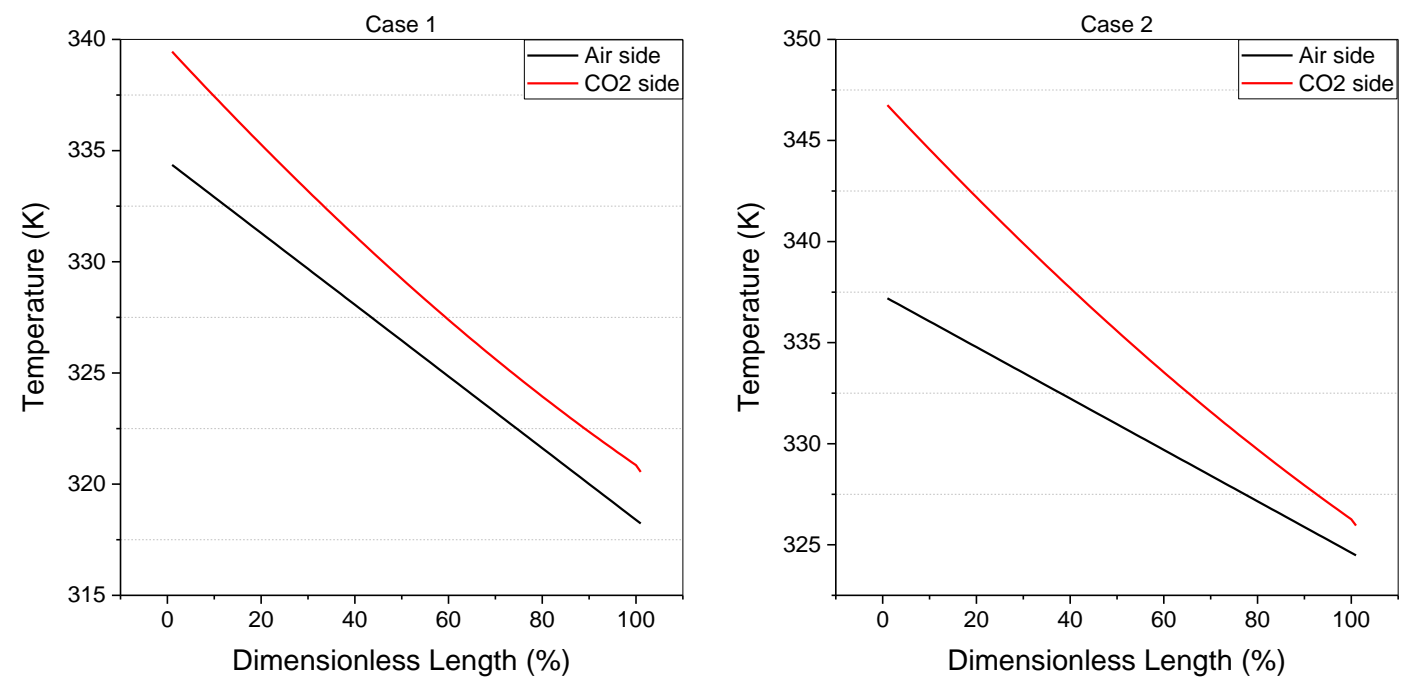

Figure 6.6. Temperature distribution for the hot and cold side for Case 1 and 2 .

For the tested PCHE, 11700 channels and the corresponding mass flow rates per channel were estimated. The Nusselt numbers for the each hot $\left(\mathrm{CO}_{2}\right)$ channel as a function of the corresponding Reynolds number are plotted in Figure 6.7. The Nusselt numbers and the friction factors are calculated using various correlations. It is observed that the Gnielinski and the correlation presented in Equation 4-15 present good agreement, in contrary with Baik correlation (Eq. 4-16) which represents more efficiently flows with higher Reynolds numbers. Therefore, in the results that are presented later for the tested PCHE the Kim correlation has been used (Eq. 4-17).

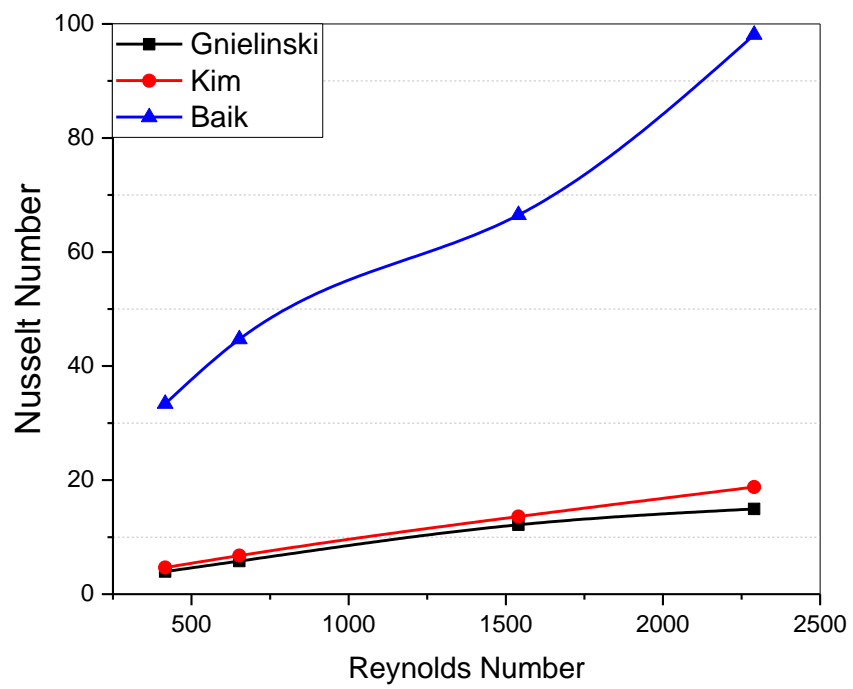

Figure 6.7. Nusselt number as function of Reynolds for various correlations. 
Furthermore, the pressure drop of the heat exchanger is of significant importance to the cycle control, but also consists a significant parameter of mechanical energy losses for the system. Figure 6.8 shows the variation of the pressure drop across a range of mass flows. A certain error in pressure measurement has also been estimated $(0.25 \%)$. The predicted pressure drop based on Table 4.3 for each case is also presented in the same figure.

The heat exchanger seems to present a reasonable pressure drop of about $2 \%$ for low mass flows, but as the mass flow increases to $0.75 \mathrm{~kg} / \mathrm{s}$ (maximum for the experiments) the pressure drop is getting considerable, resulting in a maximum pressure drop about $9 \%$ for mass flow of the last case. Therefore, as the mass flow approaches the design point, the pressure drop is in acceptable range (below 2\%), but as the operation is getting away from design point the flow presents high resistance. The numerical model seems to predict the trend of the pressure drop with acceptable accuracy (about 5-6\%) for low mass flow rates, but this difference is getting higher as the mass flow increases.

For the last case there is a difference of $60 \mathrm{kPa}$ resulting in a difference of $9 \%$. In the low mass-flow cases, the model slightly underpredicts the pressure, while in higher mass flow the model overpredicts the pressure drop. This deviations possibly comes from the assumptions and estimations regarding geometrical data that were assumed, as they are not provided by Heatric.

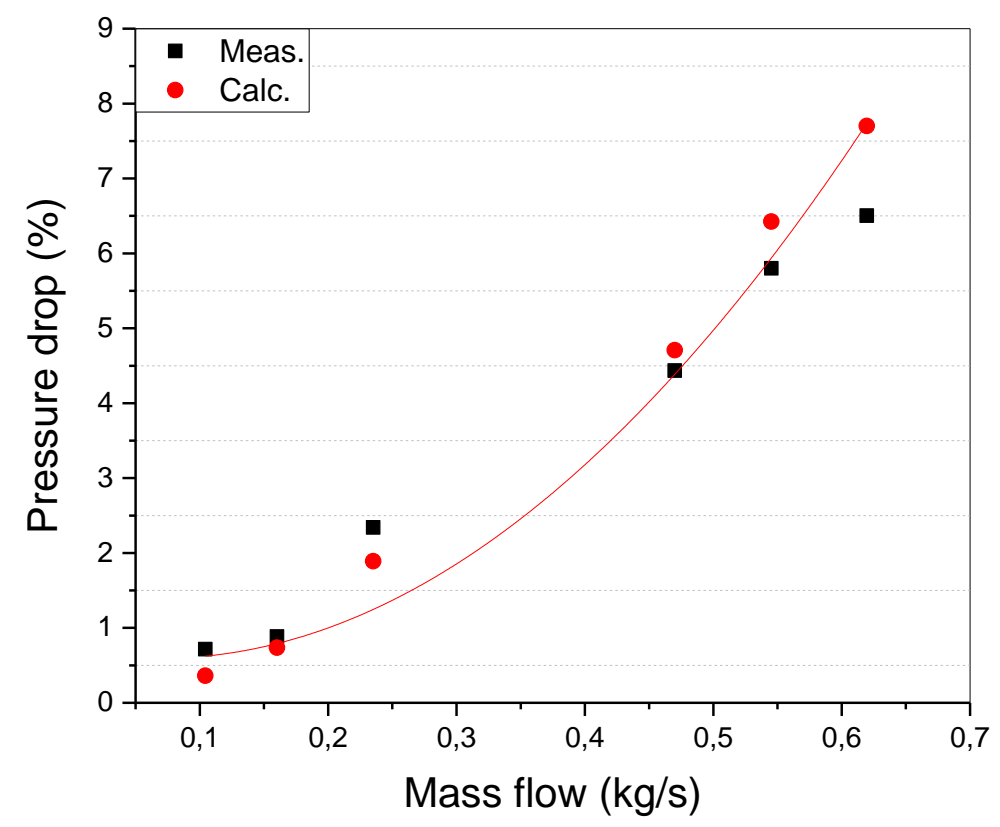

Figure 6.8. Pressure drop as percentage of inlet pressure measured cases and the corresponding predictions. 
Then, the thermal performance of the MPCHE is presented in Figure 6.9, where the computed overall heat transfer coefficient (U) for every case is shown, taking as heat transfer area the MPCHE area provided by the manufacturer $\left(12.7 \mathrm{~m}^{2}\right)$. It can be observed that the second case presents the highest heat transfer coefficient, confirming the high heat transfer rate that is presented in Figure 6.4, and the fact the measured temperatures for hot and cold sides are close enough. The overall heat transfer coefficient was based on the equation that takes into account log mean temperature difference (LMTD) $(\dot{Q}=U A \cdot L M T D)$, where LMTD is calculated as function of inlet and outlet temperatures for both heat exchanger sides. Case 4 present high overall heat transfer coefficient, even if the presented heat transfer (Figure 6.4) is not high enough, because of the high mass flow rate, far from design point.

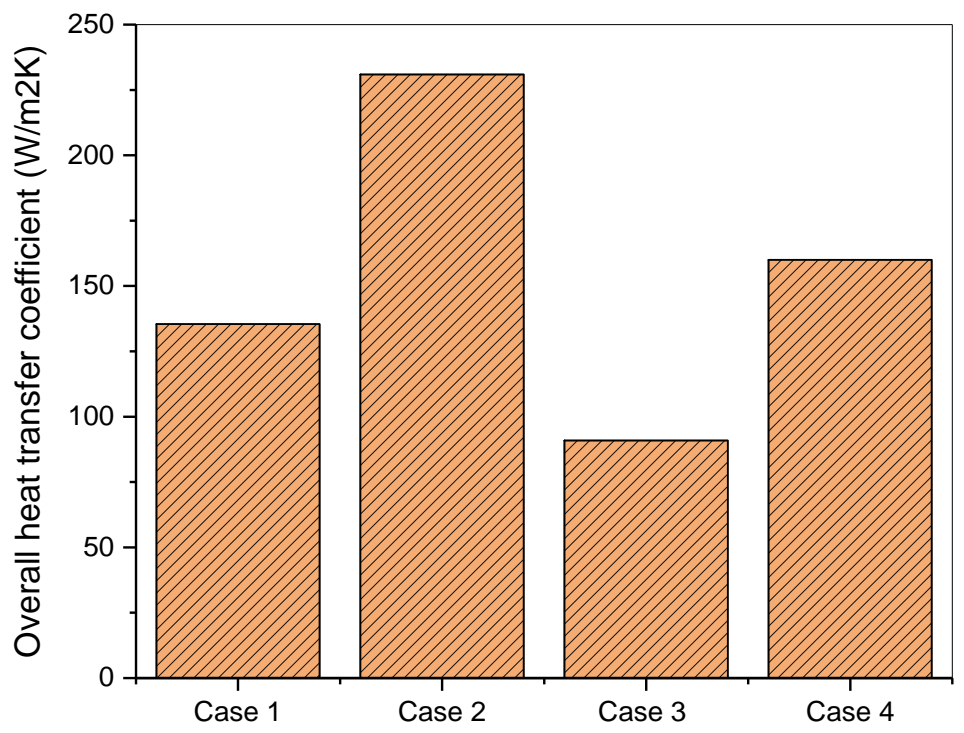

Figure 6.9. Heat transfer coefficient along heat exchangers for the four operating cases.

\subsubsection{Dynamic model results}

Various transient scenarios were investigated during the tests, in order to examine the heat exchanger's dynamic performance. A key scenario was run from a cold start simulating a power-up operation between two off-design points in the facility. The mass flow of supercritical $\mathrm{CO}_{2}$ started increasing in the heat exchanger, reaching very quickly a value about $0.6 \mathrm{~kg} / \mathrm{s}$ (Figure 6.10). The scenario simulated a quick change from cold start to $20 \mathrm{~kW}$ of thermal energy. At the same 
time, the hot-side inlet temperature started increasing from $315 \mathrm{~K}$ to a stabilized temperature in seventeen minutes, as the $\mathrm{CO}_{2}$ mass flow was already constant.

Figure 6.11 presents the experimental results for this test case, showing that the hot-side inlet temperature increased from $315 \mathrm{~K}$ to $363 \mathrm{~K}$ with a relatively fast ramp.This variation of the $\mathrm{CO}_{2}$ inlet temperature led to the corresponding $\mathrm{CO}_{2}$ outlet temperature, which is also presented in Figure 6.11. Moreover, this ramp change of inlet temperature was introduced as input to the transient model, resulting in the dynamic performance of the $\mathrm{CO}_{2}$ outlet temperature and Figure 6.11 presents the numerical simulation results that predict the experimental results, showing a good agreement between the two curves with a difference $1.5 \%$ for the stabilized temperature. The carbon dioxide outlet temperature derived by the dynamic model and was compared with the experimental data, as shown in Figure 6.11. It was observed that the numerical simulation slightly under predicted the experimental value, although the agreement between the experiment and the numerical results is good. The dynamic model predicts satisfactorily the rapid increase of the temperature during the first three minutes of the experiment, but then having reached the steady state, presents a small deviation of $1.5 \%$. As shown by Figure 6.10 and Figure 6.11, both hot-side inlet and outlet temperatures have a fast response. This is expected by the compact design of the heat exchanger.

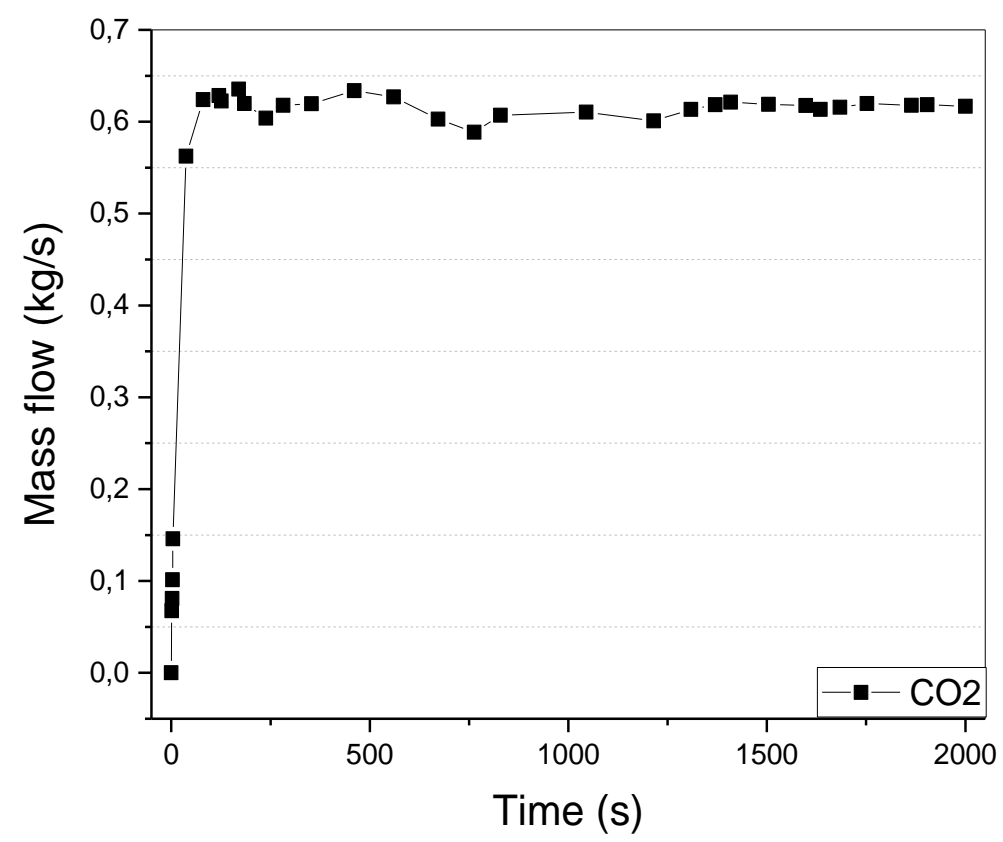

Figure 6.10. Mass flow variation with the time. 


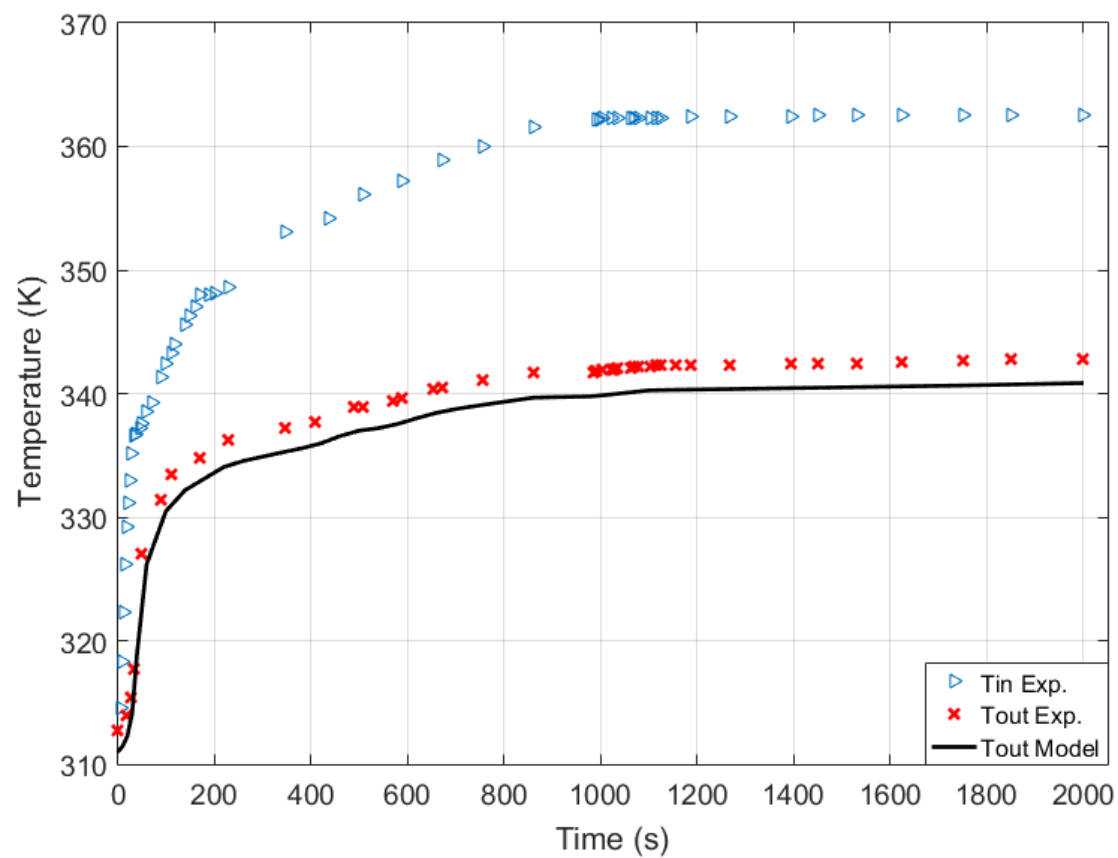

Figure 6.11. Dynamic behavior of $\mathrm{CO}_{2}$ outlet temperature for ramp variation of $\mathrm{CO}_{2}$ inlet temperature.

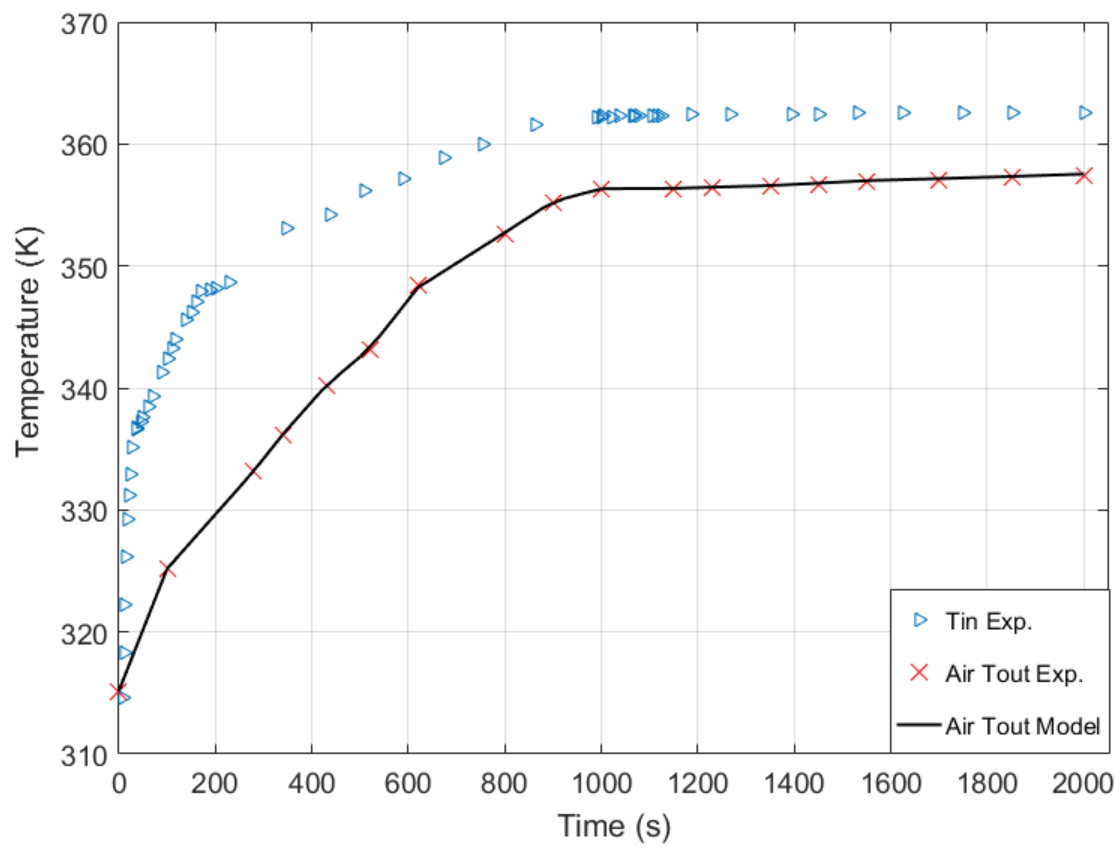

Figure 6.12. Dynamic behavior of air outlet temperature for experimental and numerical results during power-up. 
The cold-side temperature outlet of the heat exchanger is presented in Figure 6.12. During this test case, the air provided by a fan enters the heat exchanger with an almost constant temperature about $315 \mathrm{~K}$. The hot carbon dioxide heats the colder air until a steady-state condition is reached after seventeen minutes, when the air attains a temperature of $357 \mathrm{~K}$.

The difference between the $\mathrm{CO}_{2}$ inlet temperature and the air output temperature is only 4 degrees, confirming the high effectiveness ( $87 \%$ for the final steady state) of this heat exchanger type. The cold-side temperature was predicted also using the developed transient model, resulting in a very good agreement between the numerical results and the experimental measurements. Furthermore, it can be observed that the air side presents a slower response than the hot-side and this response is related with the heat exchanger internal structure.

The next transient scenario that was investigated, was a step change of mass flow. At the same time with the significant change of the mass flow rate, a step change of the temperature took place. The supercritical $\mathrm{CO}_{2}$ was running in the heat exchanger for 17 minutes until a stabilized temperature about $362 \mathrm{~K}$ occurred (Figure 6.13).

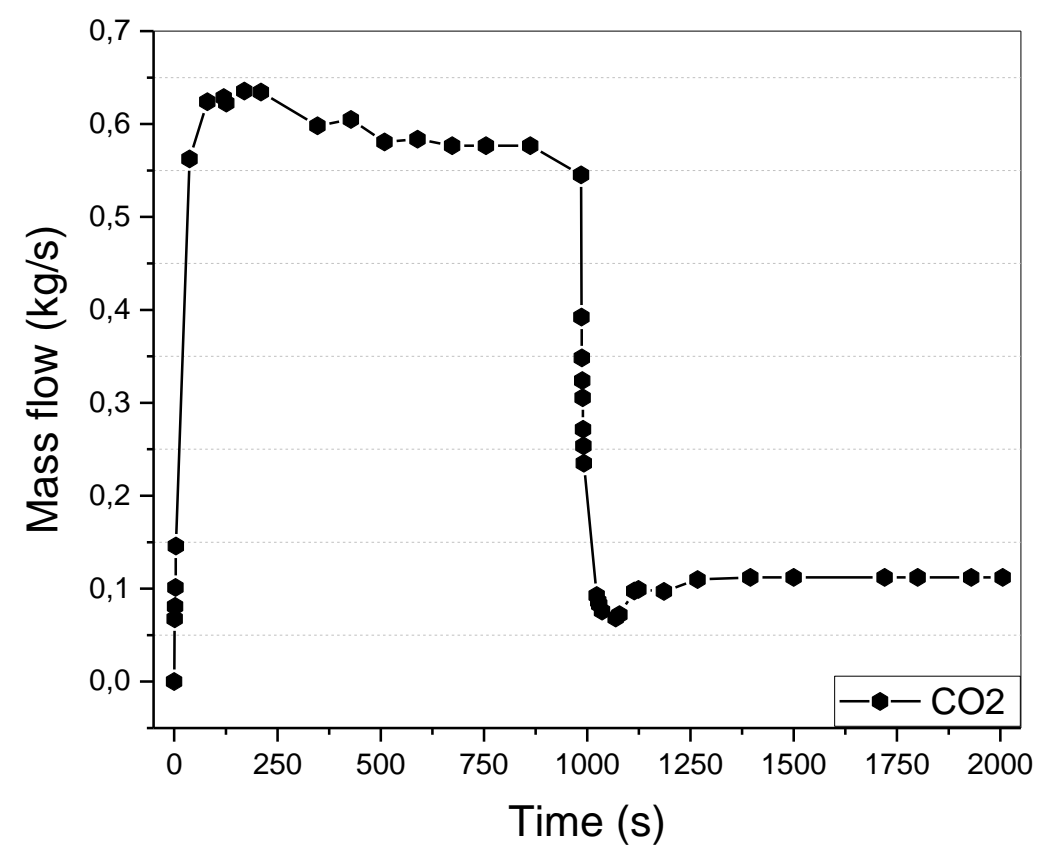

Figure 6.13. Measured carbon dioxide mass flow rates step changes. 


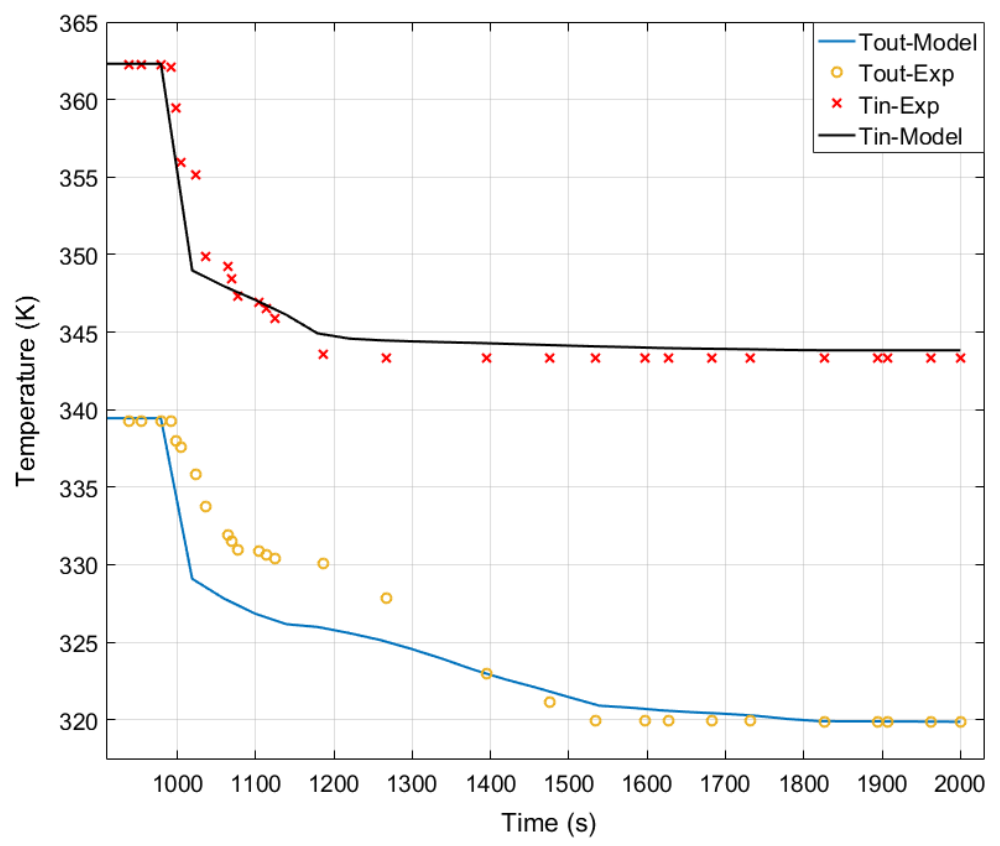

Figure 6.14. Dynamic behavior of $\mathrm{CO}_{2}$ inlet and outlet temperature for the step change.

Then the mass flow step change led in a temperature decrease with a fast ramp. The mass flow was stabilized at $0.1 \mathrm{~kg} / \mathrm{s}$, while the temperature inlet of $\mathrm{CO}_{2}$ decreased by 20 degrees, from $362 \mathrm{~K}$ to $342 \mathrm{~K}$ (Figure 6.14). At the same time, the carbon dioxide outlet temperature was decreased too, presenting a slower response than the inlet temperature. Figure 6.13 also presents the step change of the mass flow from $0.6 \mathrm{~kg} / \mathrm{s}$ to $0.1 \mathrm{~kg} / \mathrm{s}$.

For this change of mass flow rate, presents the change of the hot-side inlet and outlet temperature for both experimental measurements and results of the numerical simulations (Figure 6.14). It can be observed that the heat exchanger outlet temperature presents a slower response that the corresponding inlet temperature, which may be result of the solid plate's thermal inertia.

\subsection{Numerical Solution}

This section presents the numerical analysis that was used in order to investigate the printed-circuit heat exchangers performance, by means of computational fluid dynamics (CFD). It is divided in two parts, the first one presenting the computational analysis of the tested printed-circuit heat exchanger and the second one that presents the design and performance of a PCHE employed as hightemperature recuperator of the considered recompression s- $\mathrm{CO}_{2}$ Brayton cycle. 


\subsubsection{Numerical investigation of the tested cases}

First a validation of the results of the CFD modeling is done, against the set of experimental measurements presented in the previous section. The data used for the CFD analyses are the results of the tests with a specific PCHE for four different operation points are shown in Table 6.3. The specific tested PCHE has a total length of $0.366 \mathrm{~m}$. However, the module inserted in the numerical model is only $0.054 \mathrm{~m}$ in length (1/6 of the total length). For this reason, the module studied is assumed to belong to the exit part of the heat exchanger and thus the $\mathrm{CO}_{2}$ inlet temperature, which is a necessary boundary condition for the simulation is corrected. This correction is presented in the fourth line of Table 6.3 for $\mathrm{CO}_{2}$ side, referred as corrected inlet temperature. For the same reason, the air outlet temperature, which is employed for the validation of the CFD results, is corrected according to the scaling in the heat exchanger length and is presented in the fourth line of Table 6.3 for air side, referred as corrected outlet temperature. The temperature difference $(\Delta \mathrm{T})$ row is used for the estimation of the corrected inlet and outlet temperature for the two sides respectively, and is estimated as the temperature which corresponds to temperature change for the examined module (1/6 of the total length)

Table 6.3. Test cases validated by means of CFD.

\begin{tabular}{|c|c|c|c|c|c|c|c|c|}
\hline \multirow[b]{2}{*}{$\mathrm{CO}_{2}$ Conditions } & \multicolumn{2}{|c|}{ Case 1} & \multicolumn{2}{|c|}{ Case 2} & \multicolumn{2}{|c|}{ Case 3} & \multicolumn{2}{|c|}{ Case 4} \\
\hline & In & Out & In & Out & In & Out & In & Out \\
\hline $\begin{array}{l}\text { Mass flow rate } \\
(\mathrm{kg} / \mathrm{s})\end{array}$ & 0.1 & & 0.16 & & 0.47 & & 0.62 & \\
\hline Temperature (K) & 346.75 & 325.65 & 339.45 & 320.15 & 363.05 & 339.65 & 350.35 & 332.85 \\
\hline$\Delta \mathrm{T}(\mathrm{K})$ & 3.11 & & 2.85 & & 3.45 & & 2.58 & \\
\hline $\begin{array}{l}\text { Corrected inlet } \\
\text { temp. }(\mathrm{K})\end{array}$ & 328.76 & & 323.00 & & 343.10 & & 335.43 & \\
\hline Pressure (MPa) & 8.37 & 8.31 & 7.92 & 7.85 & 9.02 & 8.47 & 9.34 & 9.2 \\
\hline Enthalpy (kJ/kg) & 477.72 & 436.66 & 470.63 & 431.67 & 497.13 & 462.09 & 467.4 & 438.69 \\
\hline Air Conditions & In & Out & In & Out & In & Out & In & Out \\
\hline $\begin{array}{l}\text { Mass flow rate } \\
(\mathrm{kg} / \mathrm{s})\end{array}$ & \multicolumn{2}{|c|}{0.26} & \multicolumn{2}{|c|}{0.39} & \multicolumn{2}{|c|}{0.58} & \multicolumn{2}{|c|}{0.97} \\
\hline Temperature (K) & 324.60 & 341.24 & 319.20 & 335.43 & 322.90 & 351.21 & 322.07 & 340.15 \\
\hline$\Delta \mathrm{T}(\mathrm{K})$ & & 2.46 & & 2.39 & & 4.18 & & 2.67 \\
\hline $\begin{array}{l}\text { Corrected outlet } \\
\text { temp. }(\mathrm{K})\end{array}$ & & 327.06 & & 321.59 & & 327.08 & & 324.74 \\
\hline Pressure $(\mathrm{kPa})$ & 113 & 111.93 & 113 & 112 & 113 & 110.2 & 113 & 110.14 \\
\hline
\end{tabular}


Flow entrance and exit pressure losses are not taken into account, since only a part of the full heat exchanger is modeled. The following Figure 6.15 presents the temperature distribution of both supercritical carbon dioxide and air sides for Case 3. The figure illustrates the temperatures along the heat exchanger for the experimental test and the CFD module that represents only a part of the whole heat exchanger, as mentioned above.

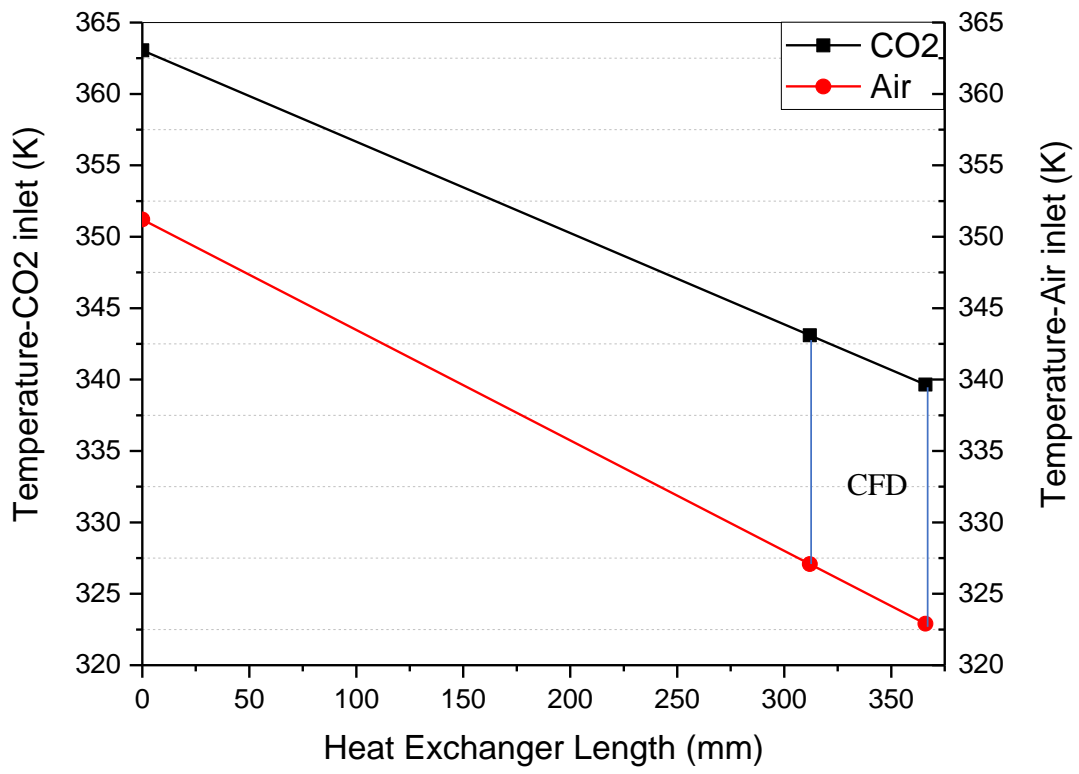

Figure 6.15. Hot and cold stream temperatures for the heat exchanger and the module for the CFD computation for test case 3 .

For the examined operational range the CFD results are presented in the following figures. Figure 6.16 and Figure 6.17 show the velocity distribution in the channels of the heat exchanger for the supercritical $\mathrm{CO}_{2}$ and air side respectively.

First of all, it is observed that the air side presents much higher velocity values (up to 200 times higher), which is explained by the higher density of the supercritical $\mathrm{CO}_{2}$ for channels of the same cross section area. Moreover, it is observed that the velocity presents high variations as the flow changes directions, which enhances the heat transfer phenomena in these areas. 


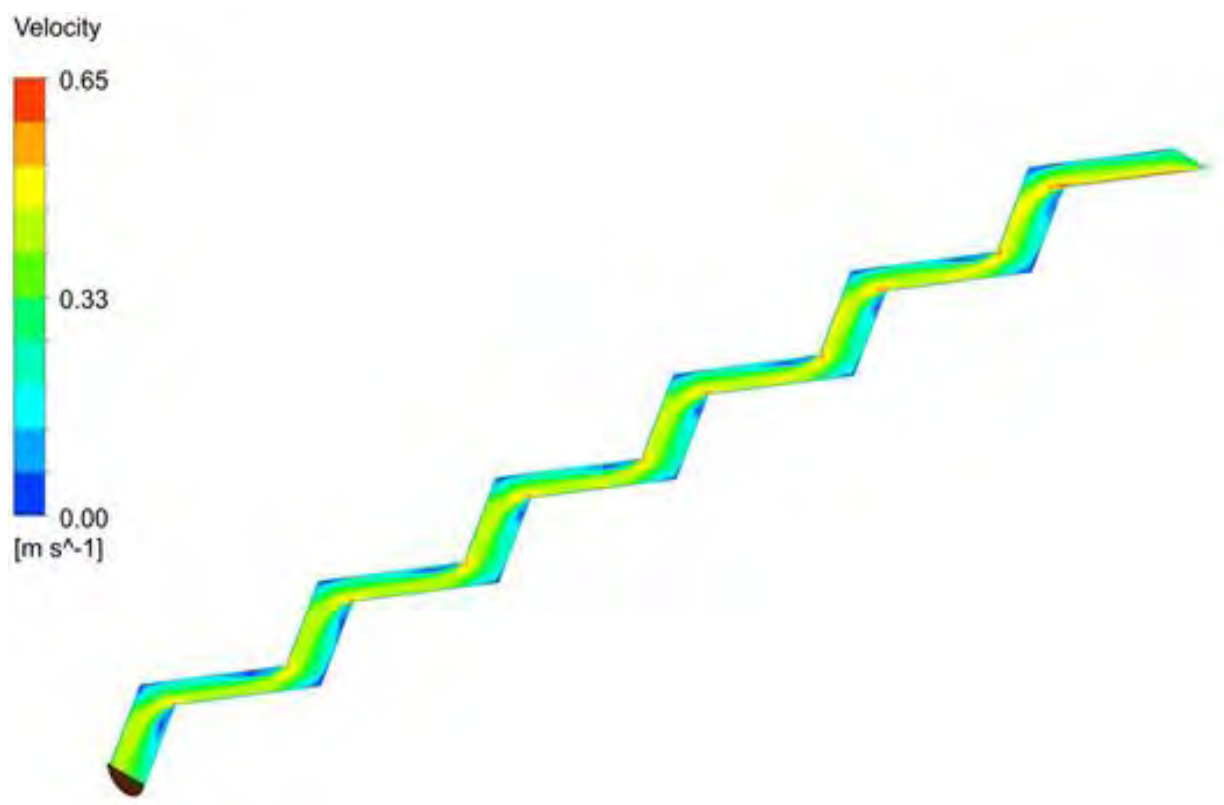

Figure 6.16. Velocity of $\mathrm{CO}_{2}$ side stream.

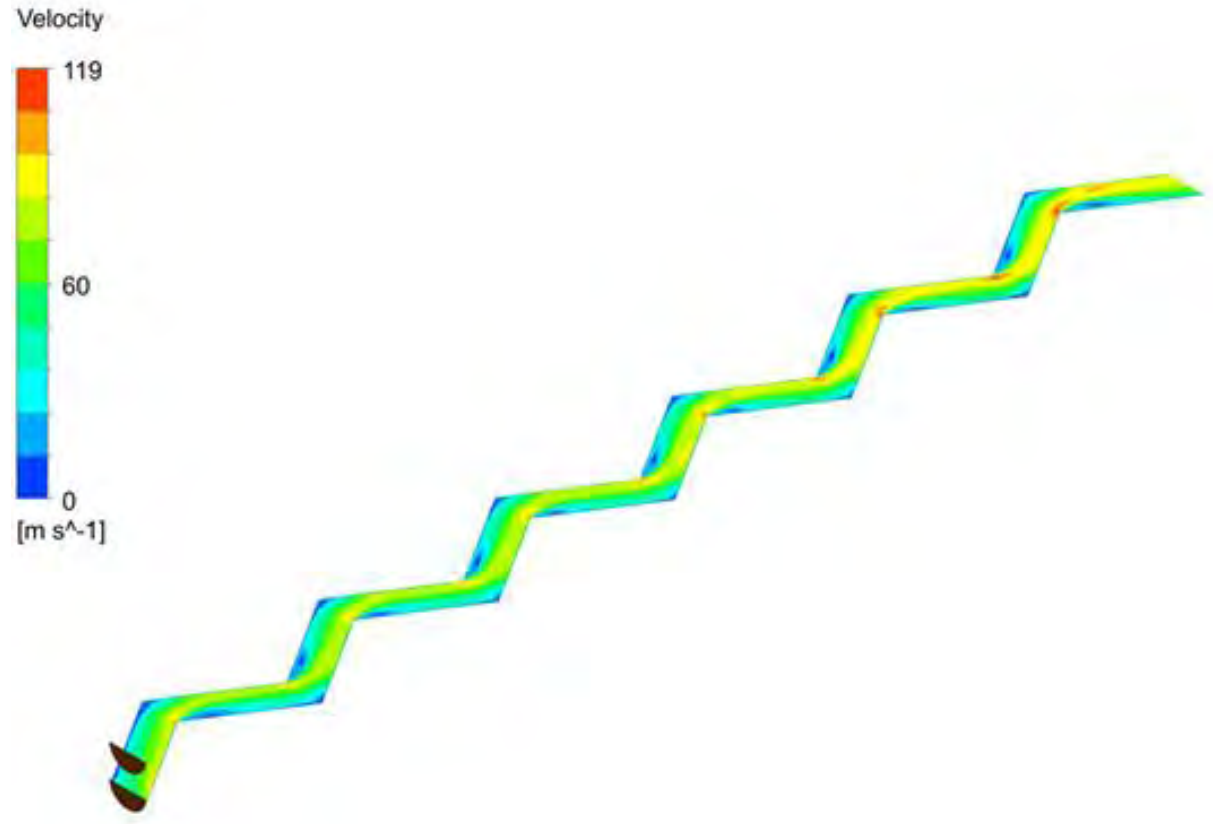

Figure 6.17. Velocity of air side stream.

Figure 6.18 and Figure 6.19 presents the temperature distribution at the outlet side for both air and $\mathrm{CO}_{2}$ sides respectively. 


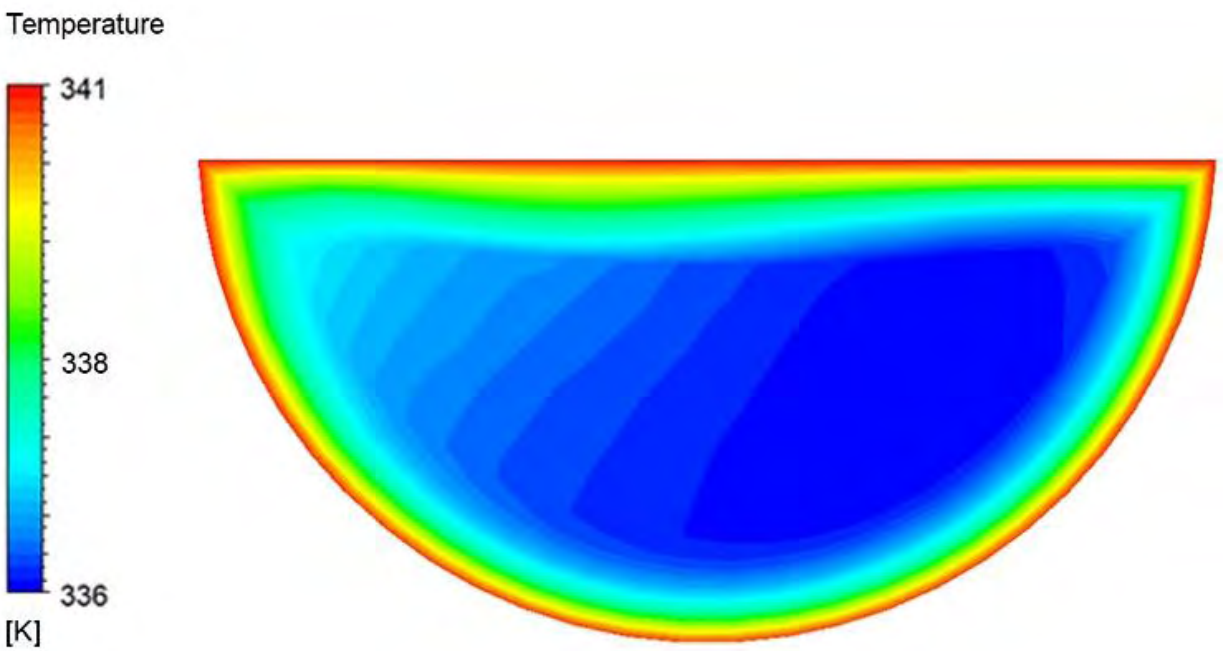

Figure 6.18. Numerical solution for outlet temperature of air side.

Temperature



$[\mathrm{K}]$

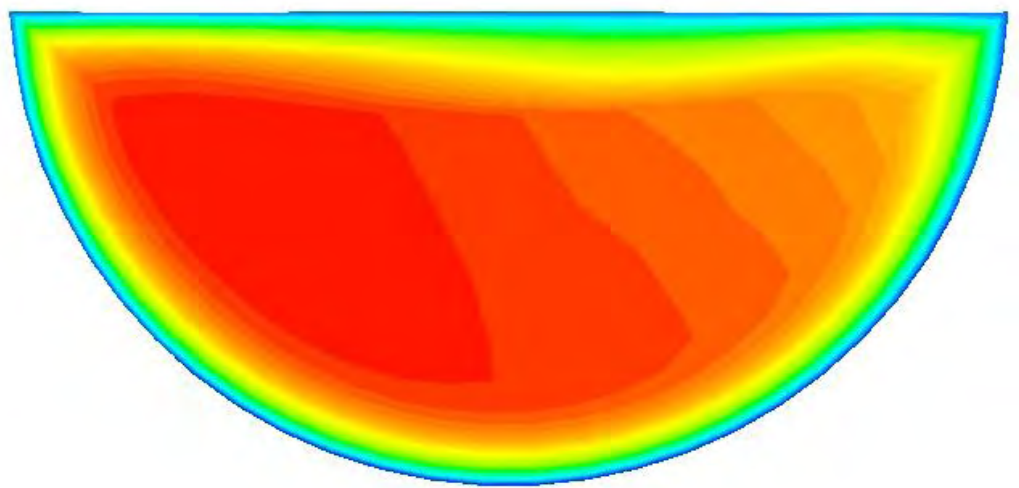

Figure 6.19. Numerical solution for outlet temperature of $\mathrm{CO}_{2}$ side.

It is observed that for air side the outlet temperature is around $336 \mathrm{~K}$, while for $\mathrm{CO}_{2}$ the outlet temperature is around $338 \mathrm{~K}$ as it is slightly hotter than air at the exit. Moreover, Figure 6.20 shows how the temperature varies along the passages for both $\mathrm{CO}_{2}$ and air sides. It can be seen that the temperature presents a similar distribution for both sides, signifying the efficient design of the heat exchanger. 


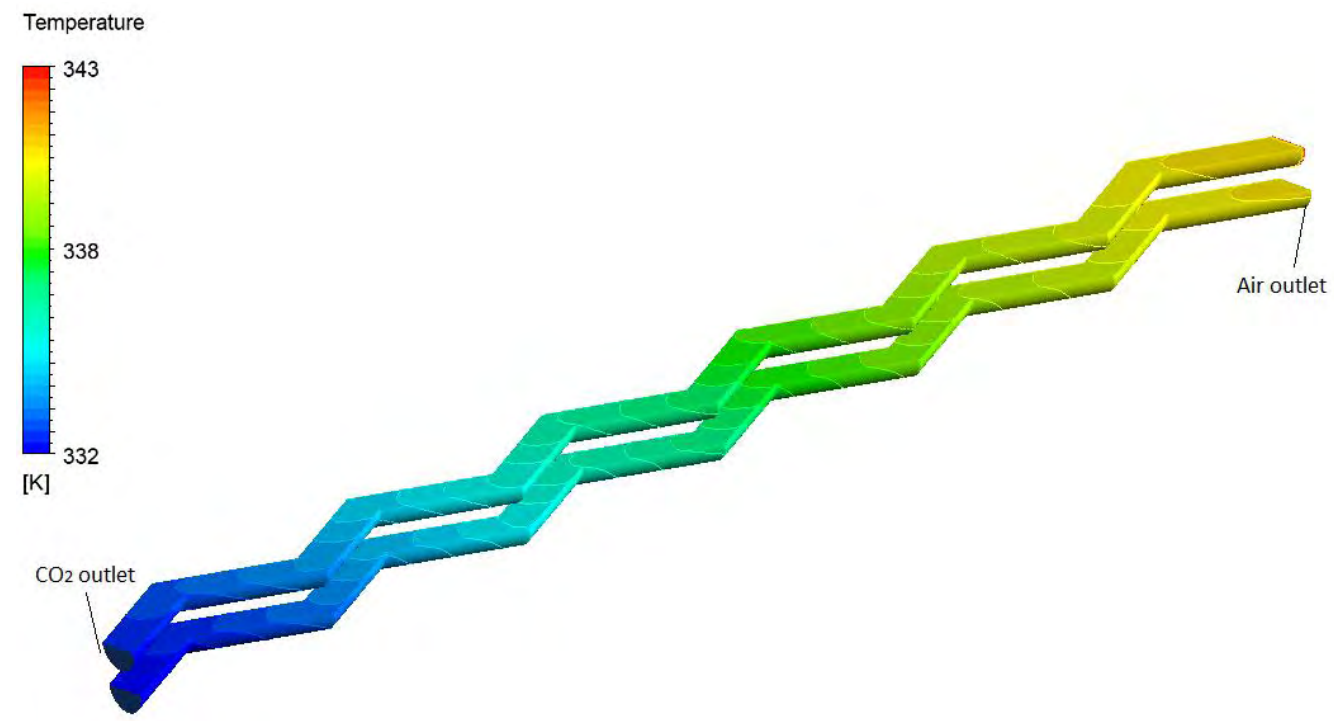

Figure 6.20. Temperature distribution in the $\mathrm{CO} 2$ and air passages along the channels.

Figure 6.21 presents the computed heat flux from $\mathrm{CO}_{2}$ to steel interface. The average value is calculated to be $2480 \mathrm{~W} / \mathrm{m}^{2}$, therefore it is estimated $(\dot{q}=U$. $L M T D$ ) that the heat transfer coefficient of the heat exchanger to be $220 \mathrm{~W} / \mathrm{m}^{2} \mathrm{~K}$. This is very close to the manufacturer's specification for the nominal operation point.

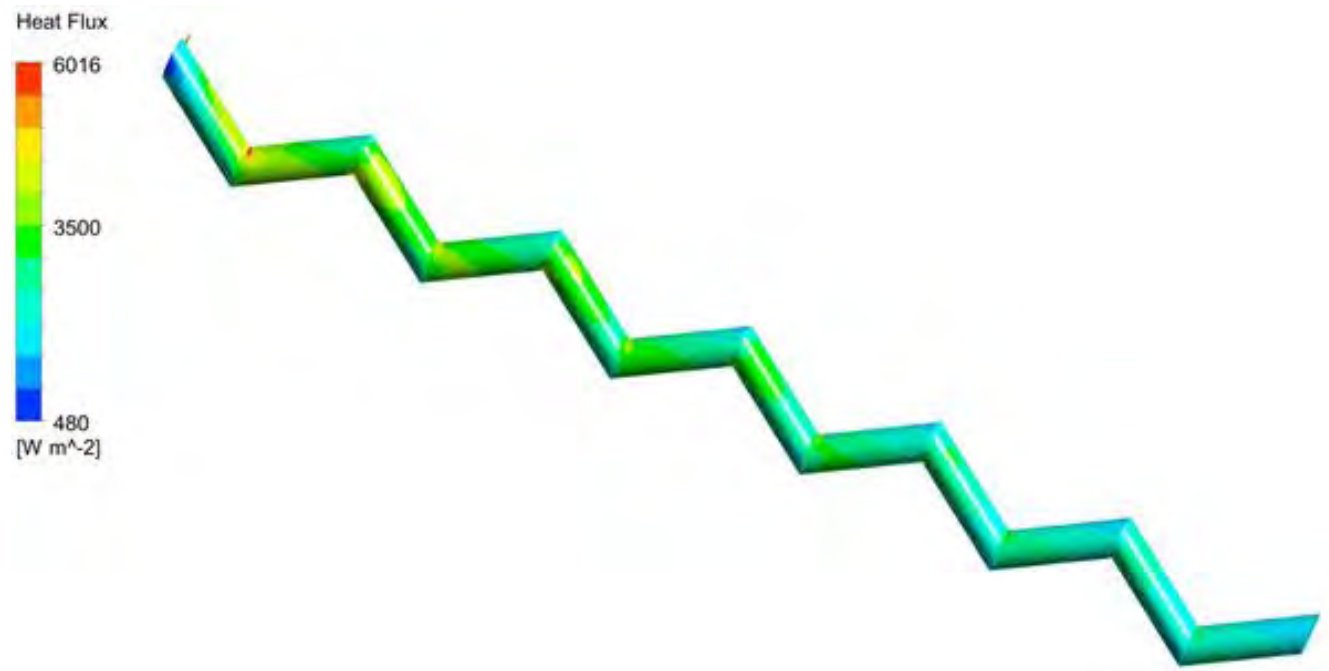

Figure 6.21. Heat flux to air interface along the channel. 


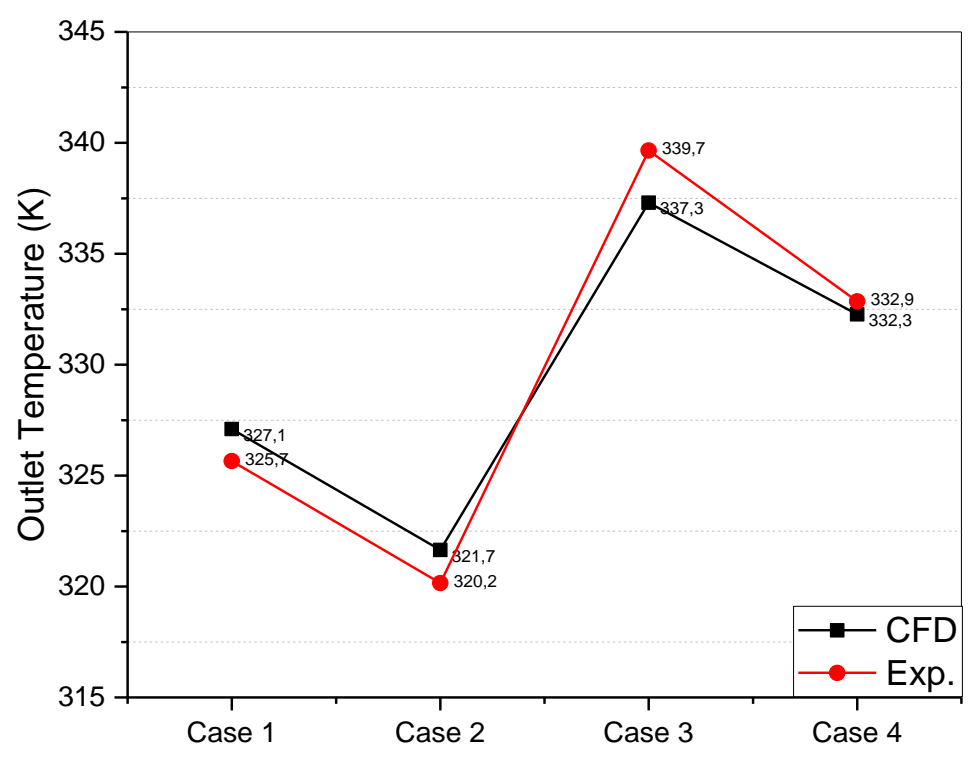

Figure 6.22. Heat exchanger outlet temperature of $\mathrm{s}-\mathrm{CO}_{2}$ for $\mathrm{CFD}$ solution and measured values.

A comparison of numerically computed and experimentally measured outlet temperatures of supercritical $\mathrm{CO}_{2}$ at the heat exchanger module are presented in Figure 6.22.

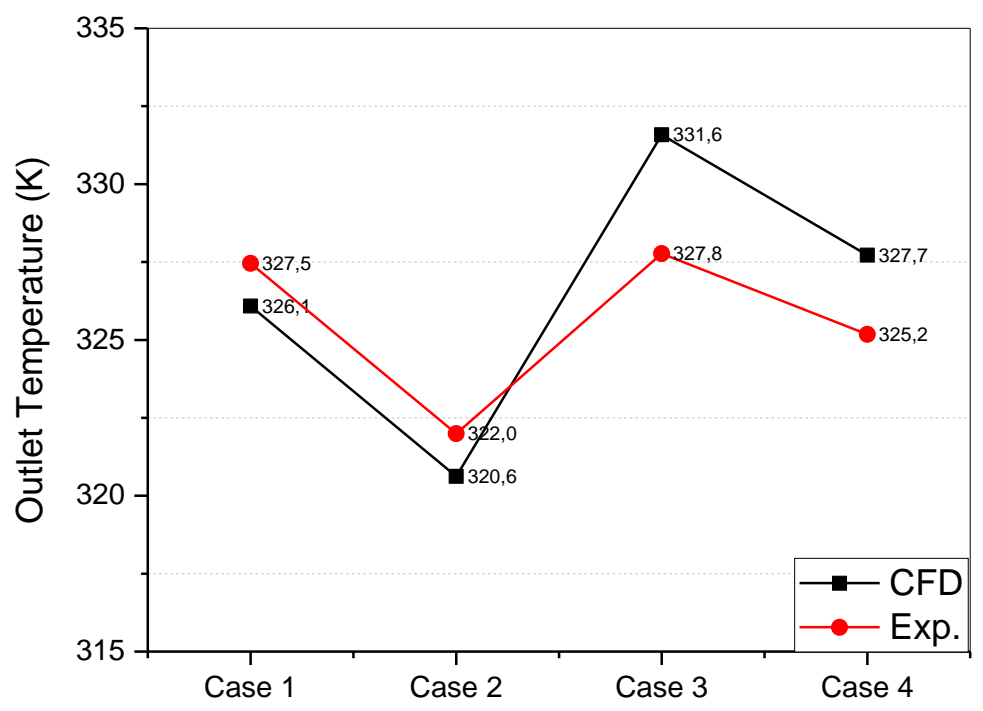

Figure 6.23. Heat exchanger outlet temperature of air for CFD solution and measured values. 
Table 6.4. Heat flux and heat transfer coefficient estimated by means of CFD for the test cases.

\begin{tabular}{lccccc}
\hline & $\begin{array}{c}\text { Heat flux } \\
\left(\mathbf{W} / \mathbf{m}^{2}\right)\end{array}$ & LMTD & $\boldsymbol{\Delta} \mathbf{T}_{\boldsymbol{\alpha}}$ & $\boldsymbol{\Delta} \mathbf{T}_{\boldsymbol{\beta}}$ & $\begin{array}{c}\text { Heat Transfer } \\
\text { Coefficient }\left(\mathbf{W} / \mathbf{m}^{2} \mathbf{K}\right)\end{array}$ \\
\hline Case 1 & 212.4 & 2.58 & 2.67 & 2.50 & 82 \\
Case 2 & 289.6 & 2.41 & 2.38 & 2.45 & 120 \\
Case 3 & 2695.0 & 12.91 & 11.52 & 14.40 & 209 \\
Case 4 & 2735.3 & 8.89 & 7.71 & 10.19 & 308 \\
\hline
\end{tabular}

Moreover, a comparison of computed and measured air exit temperatures at the heat exchanger module are presented in Figure 6.23. The differences between the experimental and CFD results for each case are considered acceptable as the maximum difference is $1.1 \%$.

Then the heat transfer was investigated. Table 6.4 presents the log mean temperature difference (LMTD) the heat flux and the heat transfer coefficient as were estimated using the numerical model for the four operating points.

A comparison of the numerically computed (measured only for design point) heat transfer coefficients for the four operation points are presented in Figure 6.24.

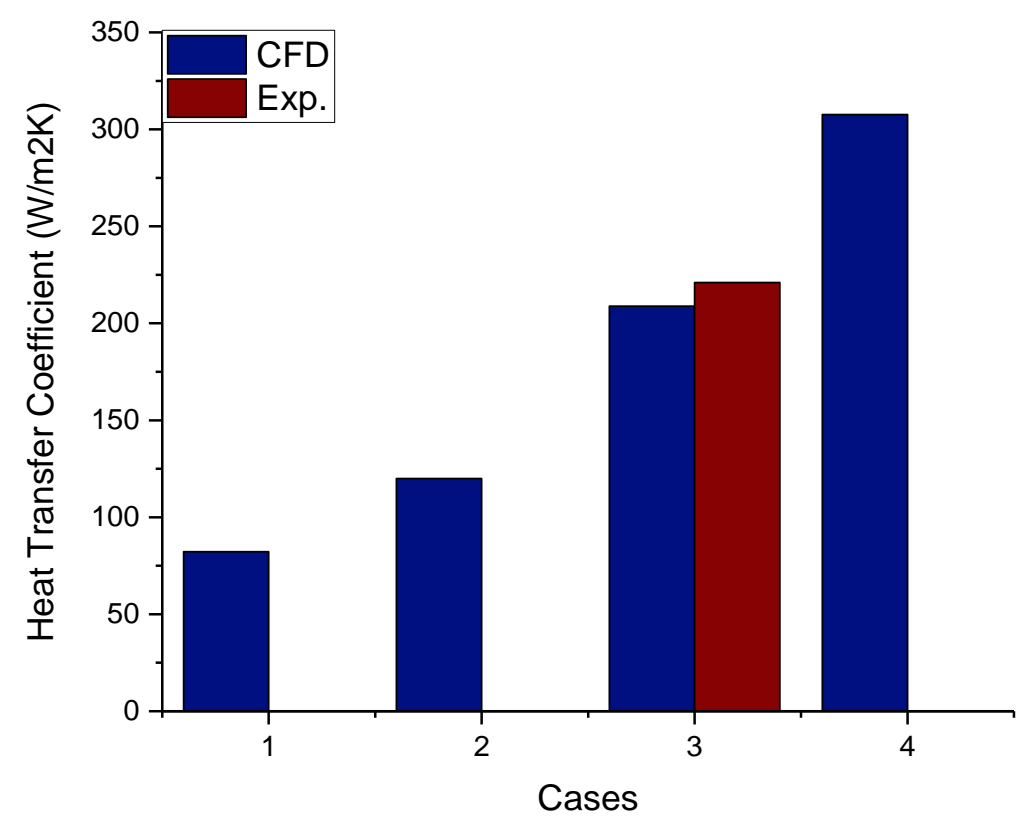

Figure 6.24. Numerically computed heat transfer coefficients for the four operation points and comparison with design point. 
Furthermore, the pressure drop for each operating point was examined numerically. A comparison of computational results and experimentally measured inlet pressures of carbon dioxide at the heat exchanger module was performed and is presented in Figure 6.25. The required inlet pressure to attain the prescribed $\mathrm{CO}_{2}$ mass flowrate against the set backpressure are systematically computed by the CFD to be significantly lower than the ones measured.

In addition, the required inlet pressure to attain the prescribed air mass flowrate against the set backpressure are systematically computed by the CFD to be higher (about double) than the ones reported by the manufacturer. This is seen by the comparison of computed and measured air inlet pressures at the heat exchanger module are presented in Figure 6.26.

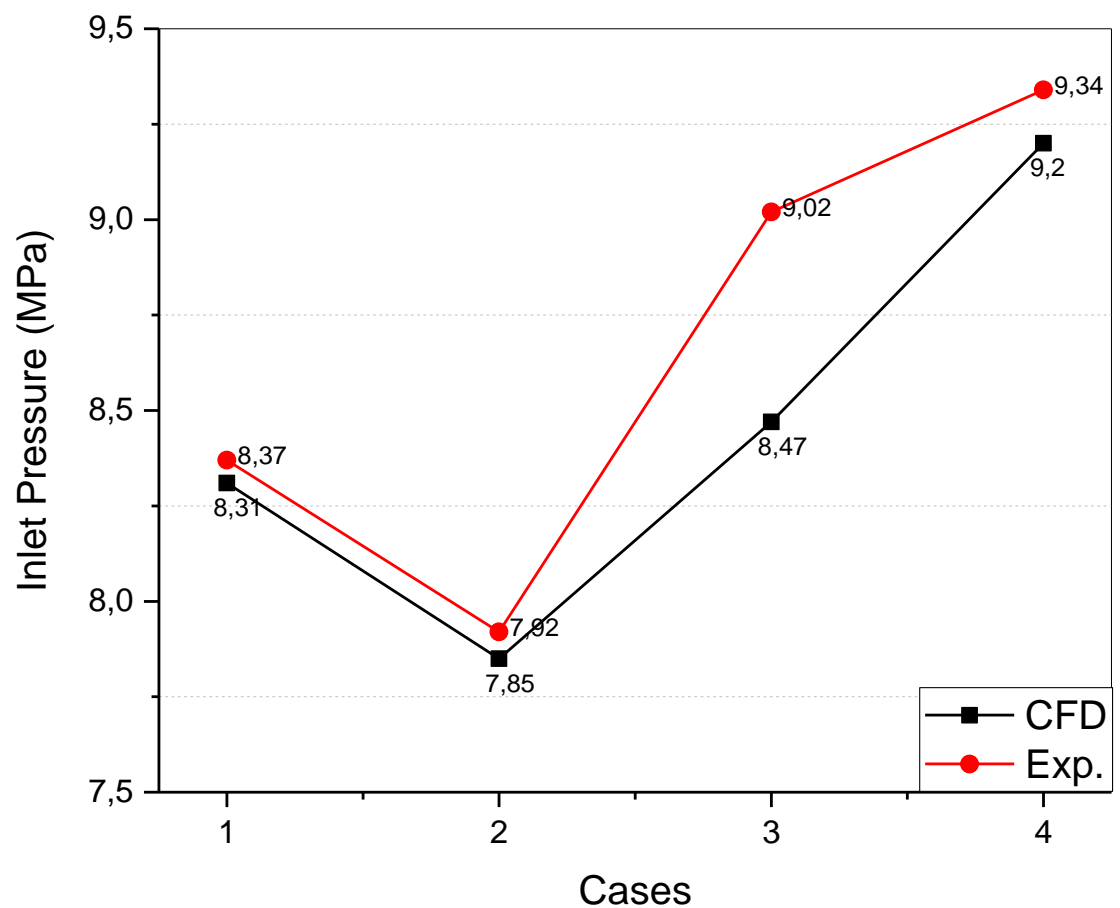

Figure 6.25. $\mathrm{S}-\mathrm{CO}_{2}$ inlet pressure derived by $\mathrm{CFD}$ and experiments. 


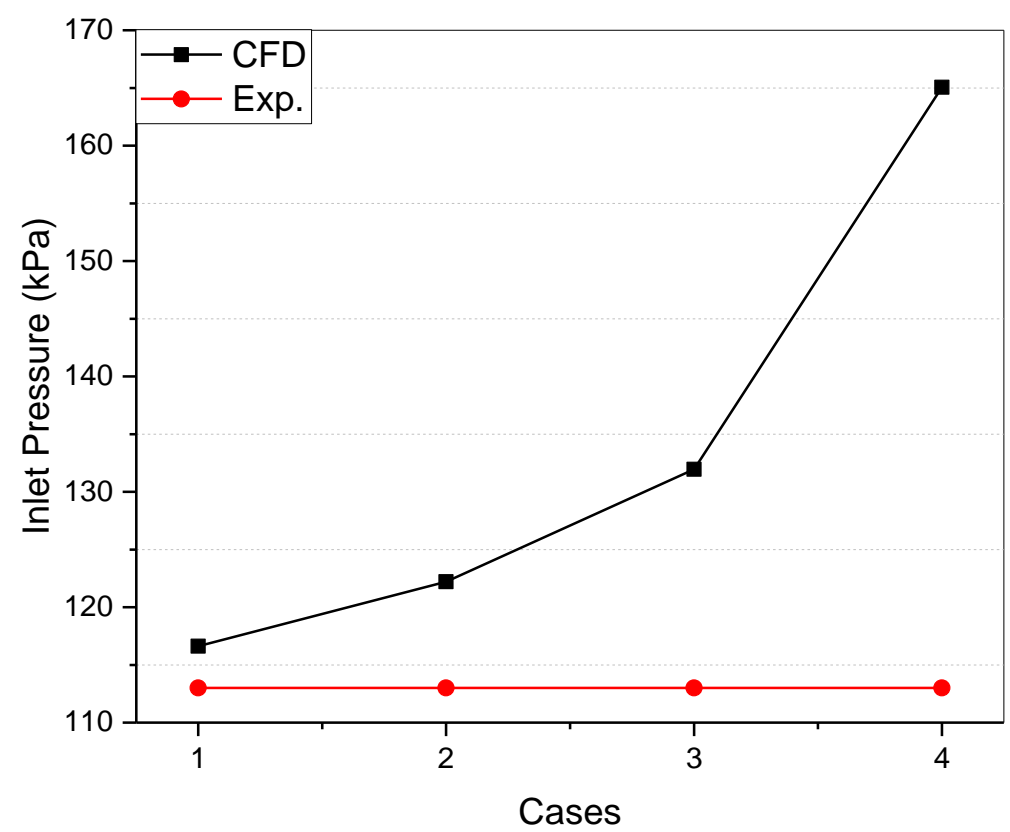

Figure 6.26. Comparison of air inlet pressure at the heat exchanger module for CFD and experimental measurements.

\subsubsection{High-temperature recuperator investigation}

Next step following the validation of the computational fluid dynamics model, is the computation of the high-temperature recuperator performance. Therefore, a final design of a high-temperature recuperator that meets the requirements of the examined recompression s- $\mathrm{CO}_{2}$ Brayton cycle will be proposed. This type of heat exchanger operates with supercritical carbon dioxide at both sides. High pressure supercritical carbon dioxide enters at 200 bar and $423 \mathrm{~K}$ and exits at $670 \mathrm{~K}$, according to the thermodynamic modeling of Chapter 3. Low pressure supercritical carbon dioxide enters at $77 \mathrm{bar}, 435 \mathrm{~K}$ and exits at $716 \mathrm{~K}$. Pressure drop is expected not to exceed $1 \%$ of inlet at both sides. Thus, high pressure circuit pressure drop should not exceed 2 bar, whereas low pressure circuit pressure drop should not exceed 0.77 bar.

The same computational module that was employed for the $\mathrm{CO}_{2} /$ air heat exchanger, will be employed in the specific computation of the HTR performance. As regards the mass-flow rate, which will be the same for both sides according to the thermodynamic cycle of Figure 3.5, this is to be set significantly higher than with the validation runs $(1.25 \mathrm{e}-4 \mathrm{~kg} / \mathrm{s})$. The reason is that the HTR of the specific 
power cycle has a high duty (around $1000 \mathrm{MW}$ ) and it is necessary for the heat exchangers to be compact, as it is the rest of the equipment.

The CFD computation will be carried out for the end right part of the heat exchanger of the Figure 6.27. The corrected high-pressure $\mathrm{CO}_{2}$ inlet temperature is set to $440 \mathrm{~K}$ and the corrected low-pressure $\mathrm{CO}_{2}$ outlet temperature is expected to be $427.5 \mathrm{~K}$ (Table 6.5). The high-temperature recuperator (HTR) length was calculated based on the design method that was presented in the previous section. Therefore, a heat exchanger total length of $3 \mathrm{~m}$ was estimated, as for the simulated part of the heat exchanger presenting a temperature difference of 5.058 needs a length $0.054 \mathrm{~m}$.

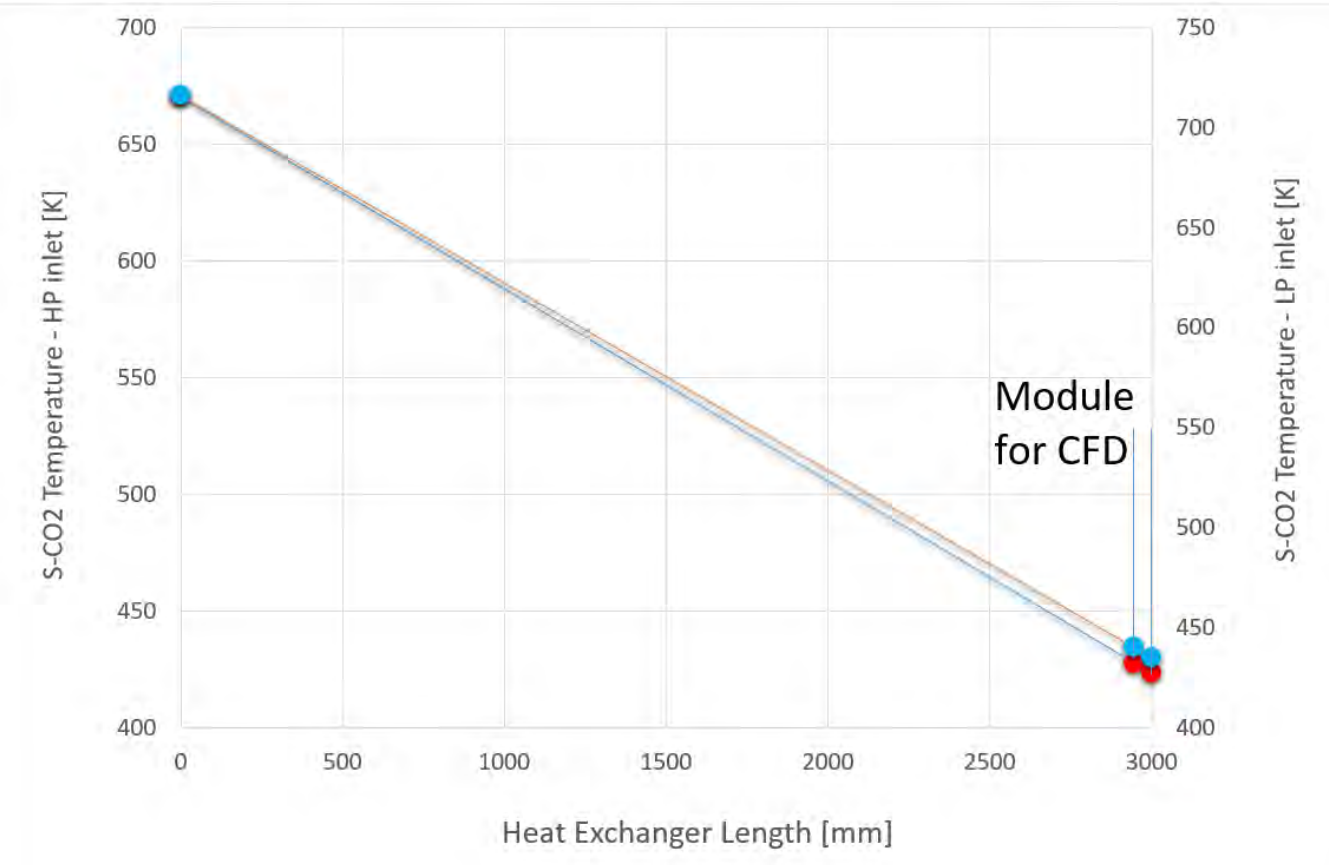

Figure 6.27. Module for the CFD computation of the full scale HTR.

The results are presented in the following diagrams. Thermal behavior seems to be predicted sufficiently well. The corresponding results for high and low-pressure sides of supercritical $\mathrm{CO}_{2}$ are presented in the following figures, presenting the pressure and the temperature. Figure 6.28 and Figure 6.29 present the temperature distribution at the outlet of the low-pressure side and high-pressure side respectively. It is shown that the average exit temperature of the low-pressure side equals $435-437 \mathrm{~K}$ as was designed, while the high-pressure side presents a temperature $426 \mathrm{~K}$. 
Table 6.5. High-temperature recuperator performance derived by means of CFD.

\begin{tabular}{lcc}
\hline Low-Pressure $\mathbf{C O}_{2}$ Conditions & In & Out \\
\hline Mass flow rate $(\mathrm{kg} / \mathrm{s})$ & 3180 & \\
Temperature $(\mathrm{K})$ & 716 & 435 \\
$\Delta \mathrm{T}(\mathrm{K})$ & 5.058 & \\
Corrected inlet temperature $(\mathrm{K})$ & 440.1 & \\
Pressure $(\mathrm{MPa})$ & 7.70 & 7.62 \\
Enthalpy $(\mathrm{kJ} / \mathrm{kg})$ & 917 & 597 \\
\hline High-Pressure $\mathbf{C O}_{2}$ Conditions & In & Out \\
\hline Mass flow rate $(\mathrm{kg} / \mathrm{s})$ & 3180 & \\
Temperature $(\mathrm{K})$ & 423 & 670 \\
$\Delta \mathrm{T}(\mathrm{K})$ & & 4.44 \\
Corrected outlet temperature $(\mathrm{K})$ & & 427.4 \\
Pressure $(\mathrm{MPa})$ & 20 & 19.8 \\
\hline
\end{tabular}

Temperature
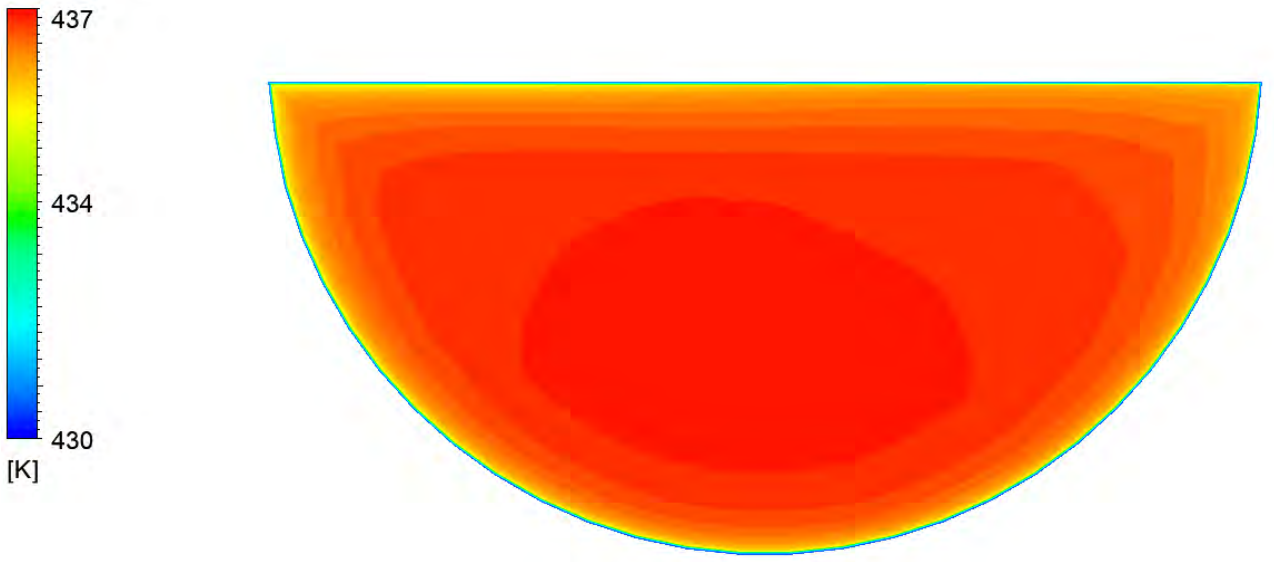

Figure 6.28. Temperature distribution at low-pressure $\mathrm{CO}_{2}$ side outlet cross section. 


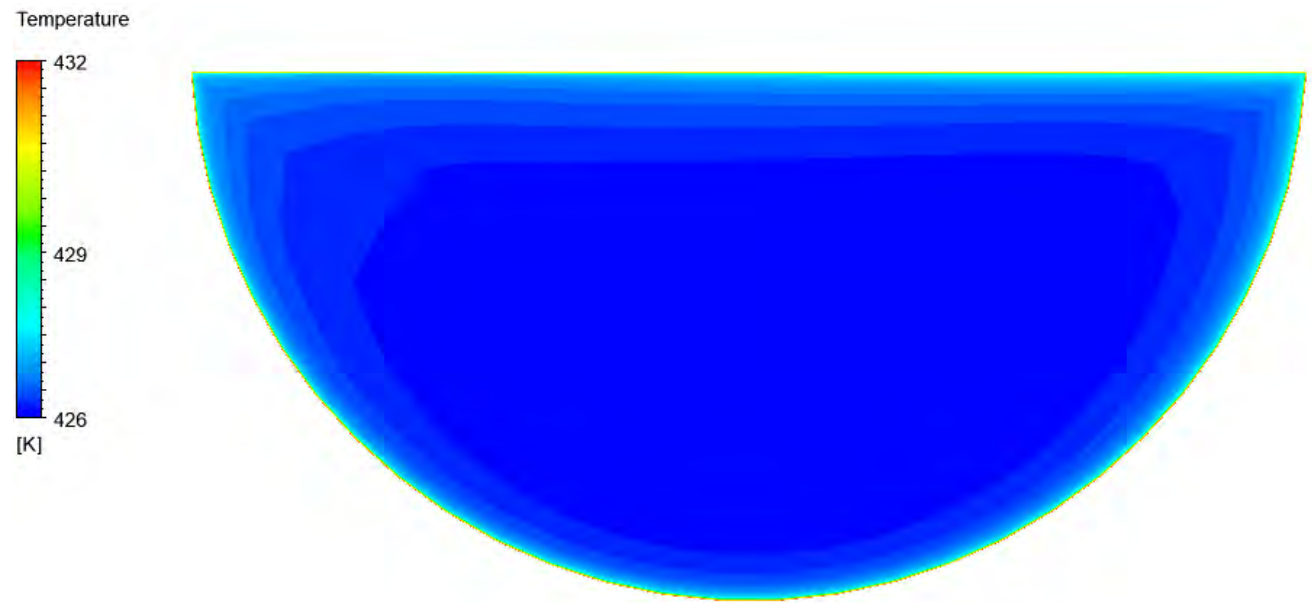

Figure 6.29. Temperature distribution at high-pressure $\mathrm{CO}_{2}$ side outlet cross section.

Moreover, the inlet pressure of both high-pressure and low-pressure sides are investigated, as the outlet pressure was imposed as boundary condition for the numerical model. Figure 6.30 and Figure 6.31 present the results for the high and low-pressure sides respectively, showing an acceptable pressure drop for the designed heat exchanger.
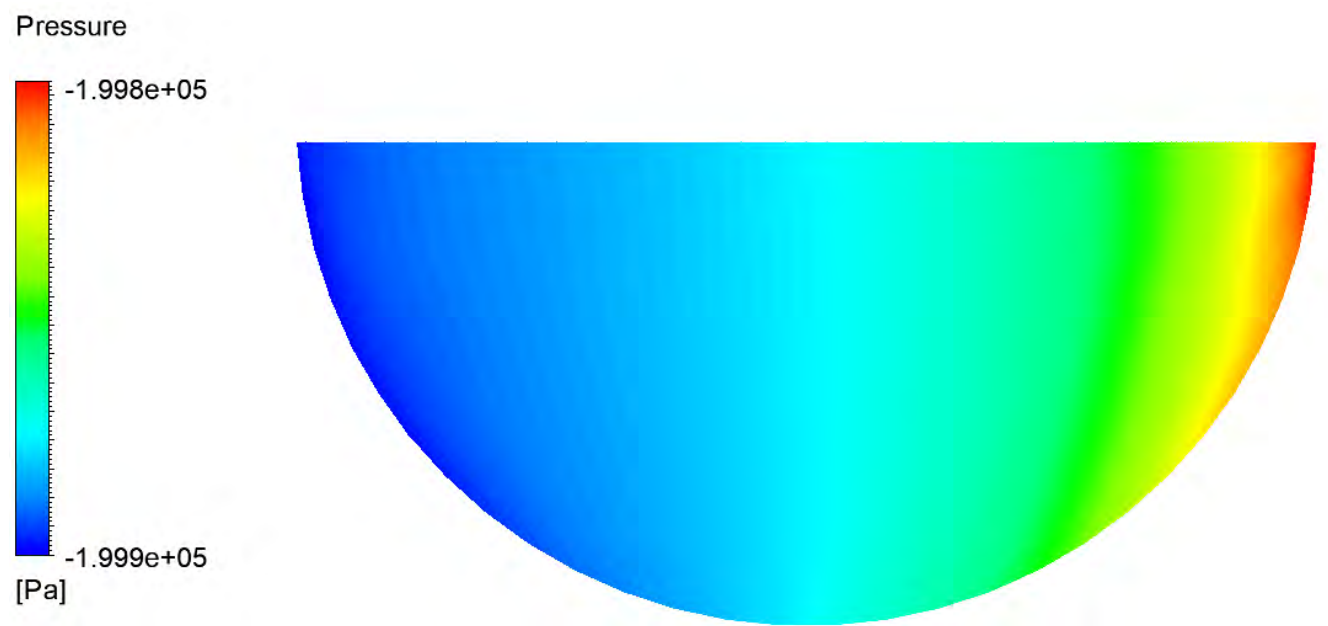

Figure 6.30. High-pressure $\mathrm{CO}_{2}$ side inlet pressure (reference pressure $20 \mathrm{MPa}$ ) 


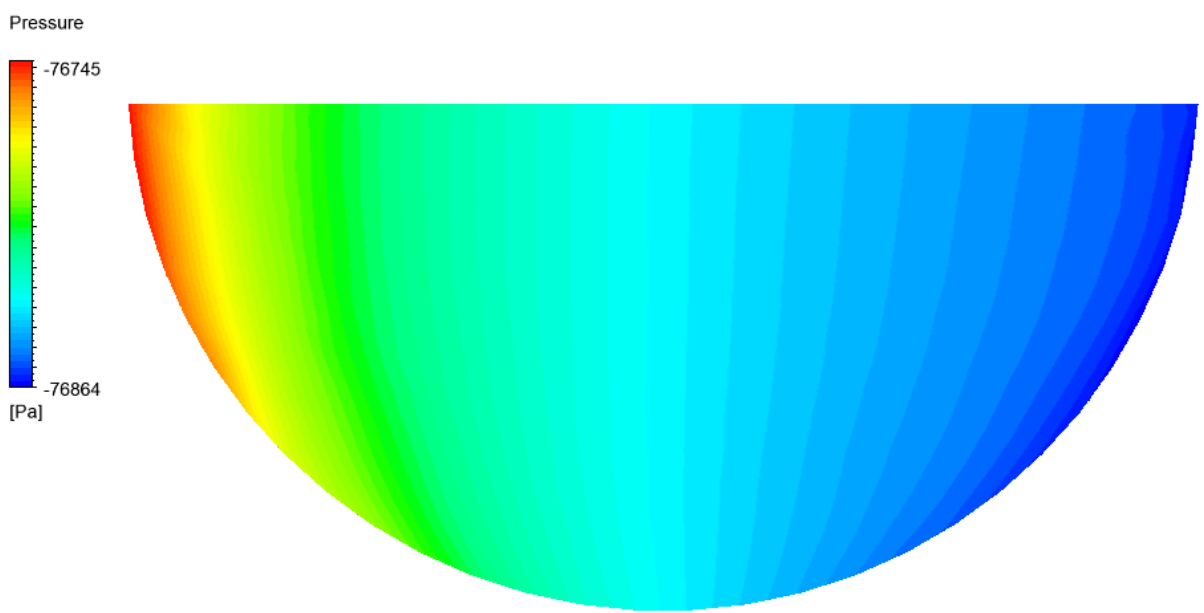

Figure 6.31. Low-pressure $\mathrm{CO}_{2}$ side inlet pressure (reference value $7.7 \mathrm{MPa}$ )

In order to investigate the performance of the specific heat exchanger design as high-temperature recuperator in the recompression s- $\mathrm{CO}_{2}$ Brayton cycle for an operational range, the CFD simulations were repeated for a total of eight operating points, varying mass flowrate per module in the range between $0.125 \times 10^{-3} \mathrm{~kg} / \mathrm{s}$ and $0.01 \mathrm{~kg} / \mathrm{s}$. For each operation point, the $\mathrm{CO}_{2}$ exit temperatures for both high and low-pressure sides are calculated, as well as the average heat flux from the hot side to the wall and from the wall to the cold side. Figure 6.32 presents the evolution of high and low-pressure side $\mathrm{CO}_{2}$ temperatures at the outlet as function of mass flowrate per module.

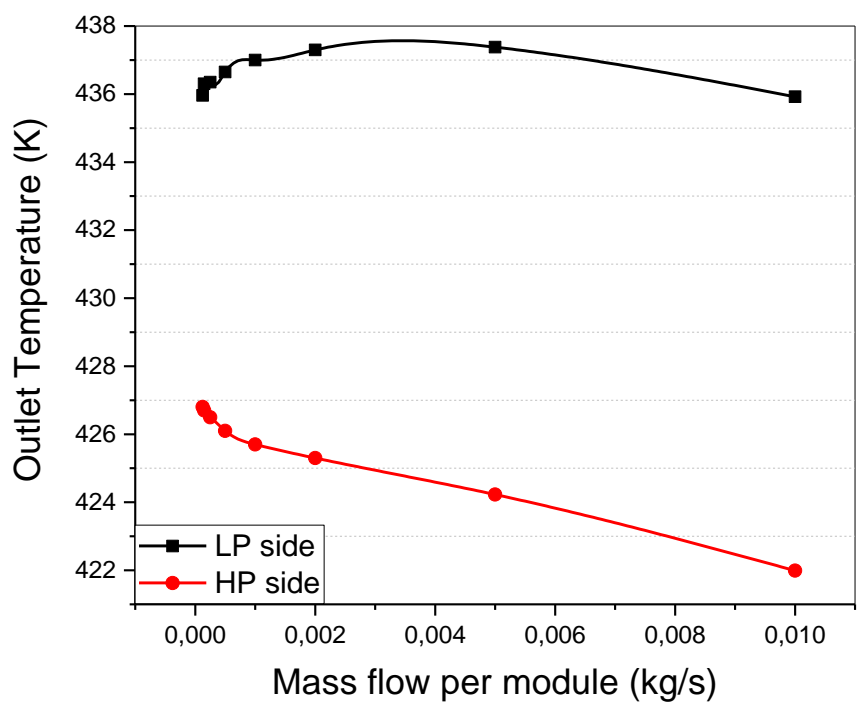

Figure 6.32. $\mathrm{CO}_{2}$ outlet temperatures for high and low-pressure side of the recuperator as calculated by the CFD 
Moreover, the logarithmic mean temperature difference (LMTD) for each point is calculated based on the known inlet and exit temperatures, as was presented in Chapter 4 . Therefore, the average overall heat transfer coefficient is calculated by dividing average heat flux by LMTD. Figure 6.33 presents the evolution of average heat transfer coefficient ( $\mathrm{U})$ as function of mass flow rate per module, as was estimated by the CFD analysis.

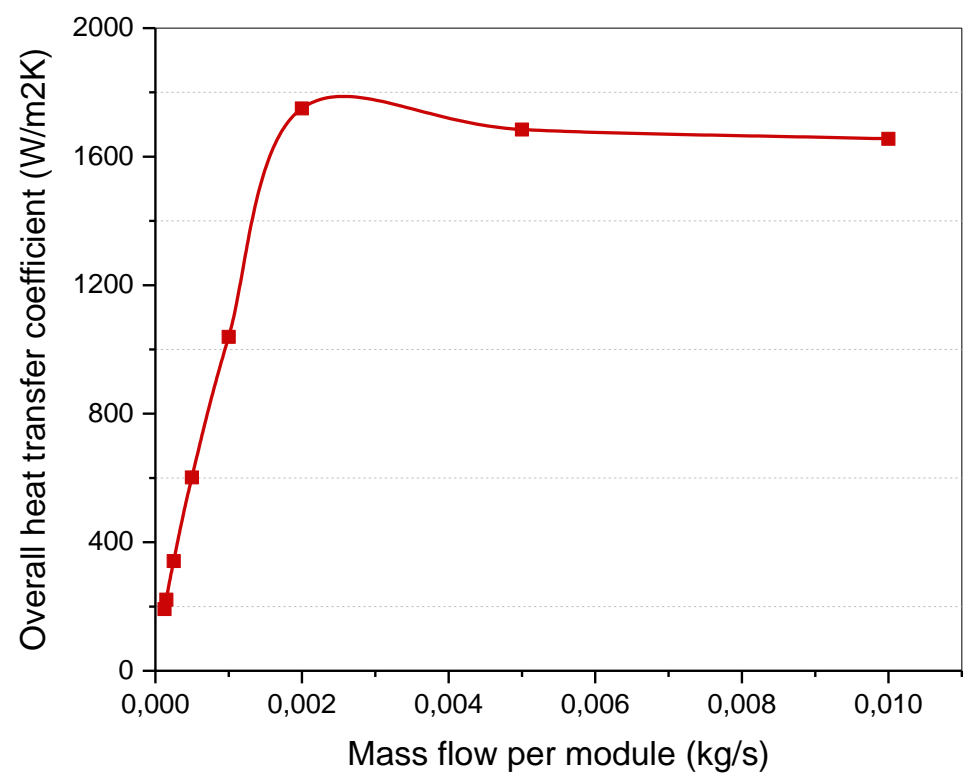

Figure 6.33. Average heat transfer coefficient for various mass flow rates per module.

The heat transfer of the designed high temperature recuperator is investigated, estimating parameters such as the number of transfer units (NTU) and the effectiveness ( $\varepsilon$ ), which were calculated using the Equations (4-16)-(4-18) and the results of NTU and $\varepsilon$ as a function of mass flow rate per module are presented in Figure 6.34. The figure shows that as the mass flow increases the number of transfer units decreases 3 units at maximum, however without having a significant impact on heat exchanger's effectiveness.

Furthermore, the pressure drop is studied, examining the inlet pressure of high and low pressure sides of carbon dioxide. Figure 6.35 presents the variation of inlet pressures of both sides as a function of mass flow rate per module, estimated by the CFD model. It is observed that the high-pressure side outlet pressure is 19.8 MPa for the first point, which corresponds to $1 \%$ pressure drop. The low-pressure side outlet pressure equals 7.62 , which also corresponds to $1 \%$ pressure drop, signifying the efficient design of the high-temperature recuperator (HTR). 


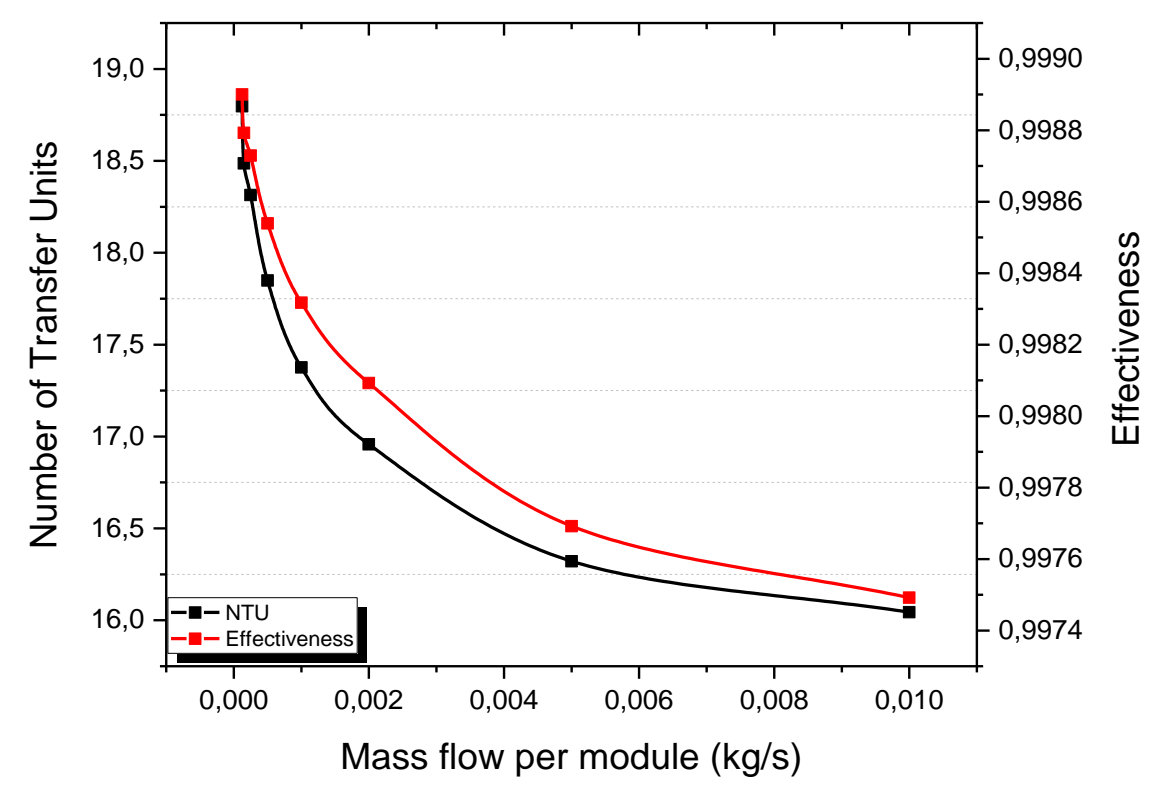

Figure 6.34. NTU and effectiveness variation as function of mass flow per module estimated with CFD.

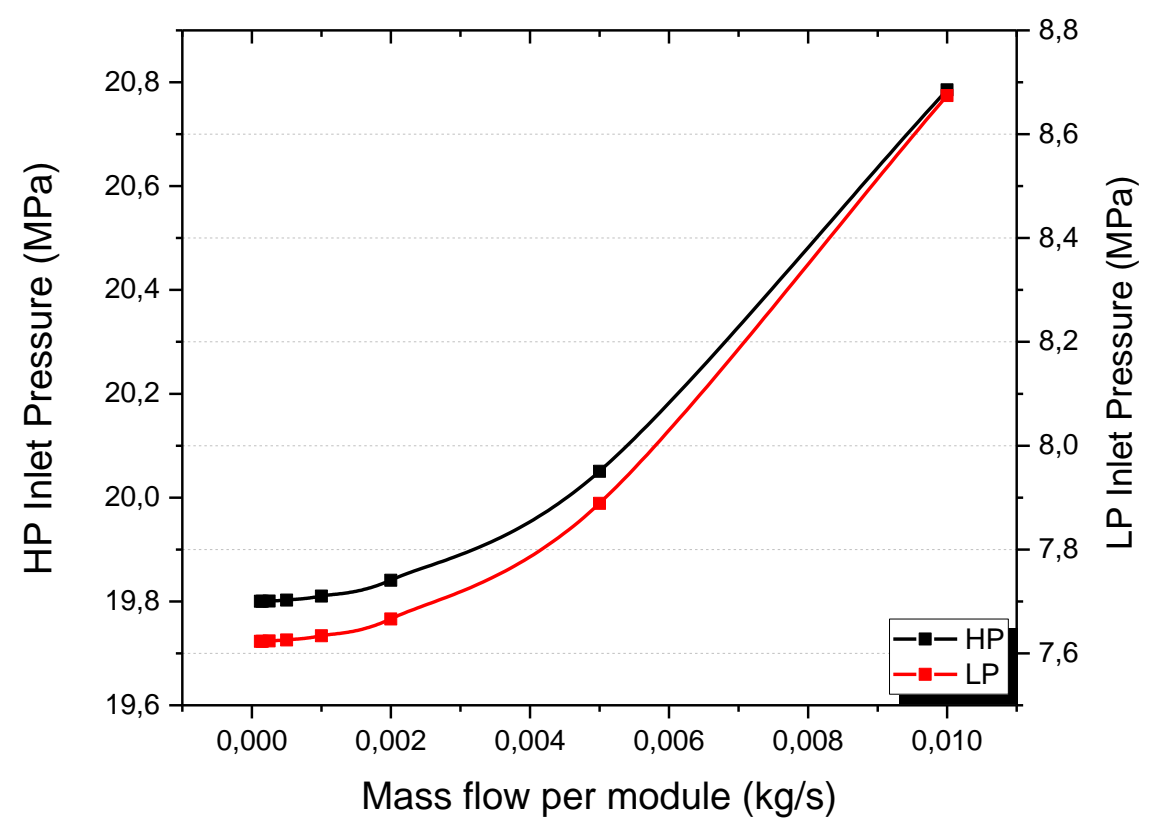

Figure 6.35. $\mathrm{CO}_{2}$ inlet pressure for high and low pressure side of designed recuperator as a function of mass flow per module and estimated with CFD. 
According to the numerical computations, the maximum permissible highpressure side $\mathrm{CO}_{2}$ pressure drop of 2 bar is attained at a mass flowrate of $0.45 \mathrm{~kg} / \mathrm{s}$. On the other hand, the maximum permissible low-pressure side $\mathrm{CO}_{2}$ pressure drop of 0.77 bar is attained at a mass flowrate of $0.3 \mathrm{~kg} / \mathrm{s}$. Thus, the optimum operation point is expected to be at a mass flowrate of $0.3 \mathrm{~kg} / \mathrm{s}$. The total heat exchange area of the specific HTR for this operation point is calculated at about $30000 \mathrm{~m}^{2}$ (Figure $6.36)$.

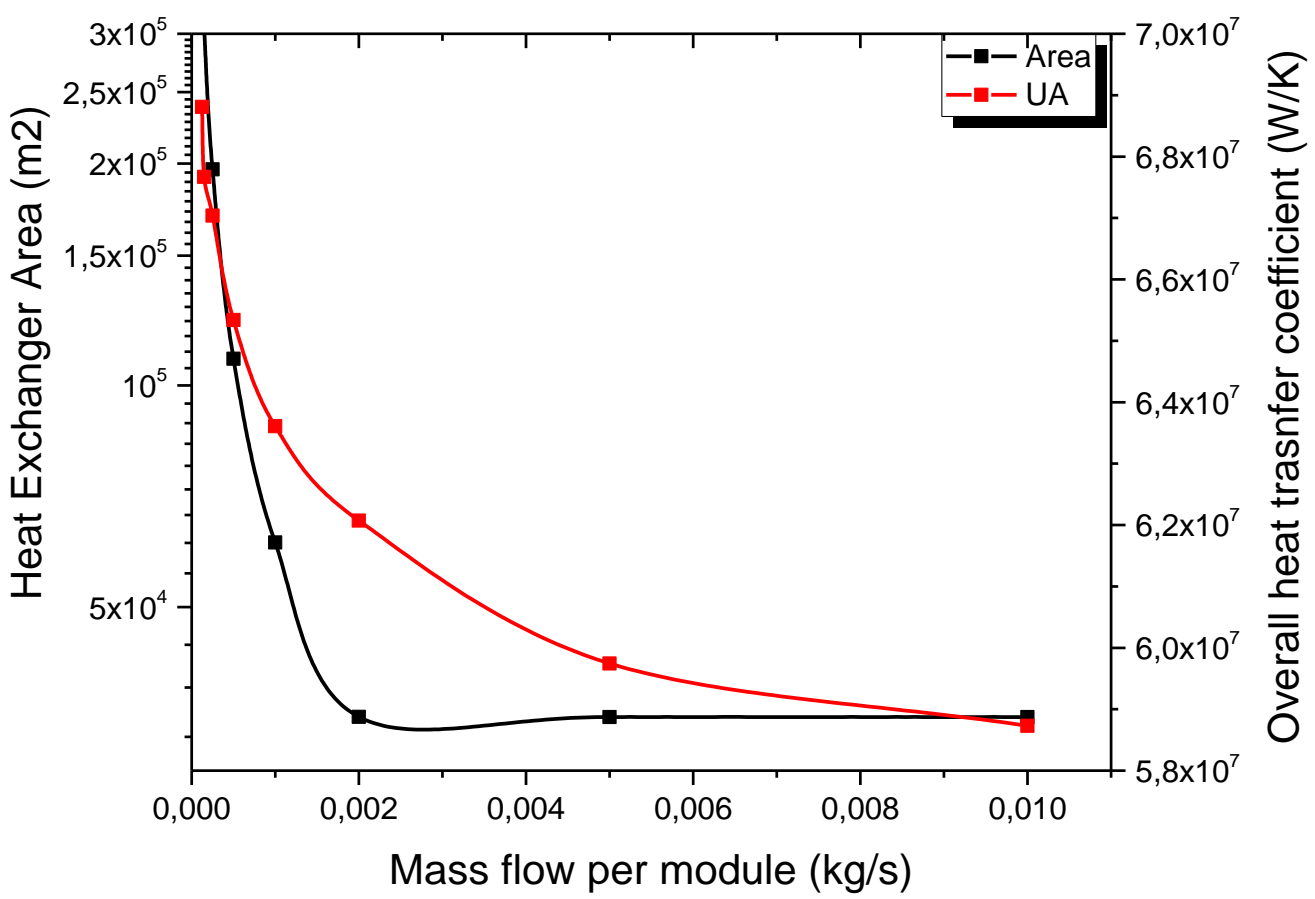

Figure 6.36. Total heat exchange area and overall heat transfer coefficient of the specific HTR as function of mass flowrate per module, calculated by the CFD.

Reynolds numbers and the corresponding Nusselt numbers were calculated for the various mass flowrates per module for the designed high-temperature recuperator. Figure 6.37 presents this variation of Nusselt number as a function of Reynolds number for the high-pressure side of the HTR. The calculation of Nusselt number was based on the Equations 4-14. The low-pressure side presents a similar variation of Nusselt number, presenting a bit lower value but is not illustrated in this figure. This difference is linear variation of the $9 \%$ divergence of viscosity between high and low pressure side of $\mathrm{CO}_{2}$. The CFD results show to have good agreement with the results calculated by the one-dimensional model for the design point (where Reynolds number equals about $3.5 \times 10^{3}$ ). 


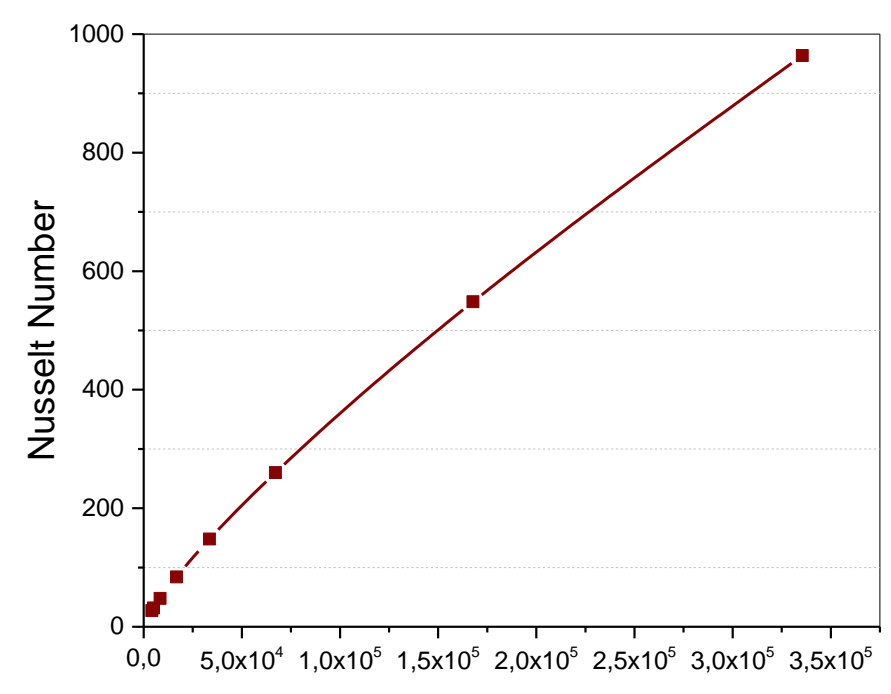

Reynolds Number

Figure 6.37. Nusselt number as function of Reynolds number for the designed HTR.

The final geometrical characteristics of the designed HTR are presented in the following table.

Table 6.6. Geometrical characteristics of the designed high-temperature recuperator.

\begin{tabular}{ll}
\hline Parameters & Values \\
\hline Length $(\mathrm{m})$ & 3 \\
Heat exchange area $\left(\mathrm{m}^{2}\right)$ & $30 \times 10^{3}$ \\
Number of channels & $318 \times 10^{3}$ \\
Channel diameter $(\mathrm{mm})$ & 1.9 \\
Pitch $(\mathrm{mm})$ & 2.2 \\
Thickness $(\mathrm{mm})$ & 2 \\
\hline
\end{tabular}

Finally, the improved effectiveness (0.99) of the designed HTR results to a higher outlet temperature of the heat exchanger compared to the simulated recompression cycle that simulate a heat exchanger with effectiveness 0.9 . This improved effectiveness leads to a higher temperature that enters the system's heater and as the heat addition is less, the cycle efficiency improves. 


\subsection{Conclusions}

In the current chapter, the investigation of printed-circuit heat exchangers took place. The results derived by the one-dimensional analysis were presented, while an experimental facility developed for testing a prototypic design printed-circuit heat exchanger employing supercritical carbon dioxide was presented. Various test cases were investigated, examining the steady state and the transient performance of the heat exchanger for an operation range that was not considered previously in literature. Furthermore, a computational fluid dynamics analysis of the tested printed-circuit heat exchanger was performed and then the high-temperature recuperator for the considered recompression $\mathrm{s}-\mathrm{CO}_{2}$ Brayton cycle was investigated.

Finally:

- The thermal-hydraulic performance of a modified printed-circuit heat exchanger was investigated for on and off-design operation.

- A dynamic model was developed and validated by the experimental results for various transient scenarios, such as power-up of the system until it is stabilized or step-change of mass flow. The results show a satisfactory agreement with the experiment, resulting in a maximum difference about $4 \%$ for the temperature outlet of supercritical carbon dioxide.

- The heat transfer coefficient validation of the tested PCHE resulted in a 5\% difference between CFD and experiments.

- The CFD analysis showed limited pressure drop for both sides of the tested PCHE.

- A final design of the high-temperature recuperator for the considered power cycle was proposed. The HTR was designed based on the previous validated CFD method. The numerical results showed effectiveness close to $99 \%$ for pressure drop up to $1 \%$ for both sides of the HTR. 


\section{Chapter 7}

\section{Centrifugal Compressor Design and Analysis}

This chapter presents the results regarding the design of the compressor stage and the computational investigation regarding its performance. Firstly, the results of the direct problem are presented, in order to show that the used methods presented in Chapter 5 predict sufficiently the outlet conditions of a tested compressor. Then, the results of the geometry derived by the inverse problem are presented. Moreover, the aerodynamic analysis examining the three-dimensional flow takes place, taking into account the real gas approximation on the numerical results. Consecutively, the possibility of two-phase flow at the blade regions with higher risk is investigated and the resulted performance map is presented.

\subsection{Direct Problem Validation}

The developed model presented in Chapter 5.1, is validated by Model A geometry, which represents the geometry data of a tested centrifugal compressor $[28,79]$. Figure 7.1 presents the tested compressor.

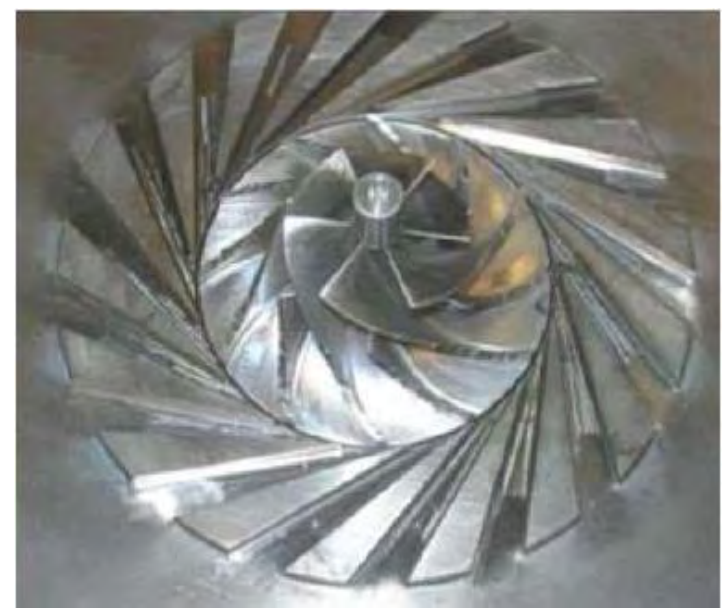

Figure 7.1. Geometry of the tested Model A [116].

Table 7.1 concentrates some significant geometrical characteristics of the Model A that used as inputs for the developed model simulating the direct problem. The main scope of the direct problem solution is the validation of the model 
incorporating the losses that take place inside the compressor, in order to estimate with acceptable accuracy the pressure outlet.

Table 7.1. Centrifugal compressor geometry Model A, used as input for direct problem.

\begin{tabular}{ll}
\hline \multicolumn{2}{c}{ Model A Geometry } \\
\hline Number of main blades & 6 \\
Number of splitter blades & 6 \\
Impeller inlet radius at hub (mm) & 2.5 \\
Impeller outlet radius at tip (mm) & 9.4 \\
Blade angle of impeller leading edge at mean (deg) & 39.5 \\
Blade angle at impeller exit (deg) & -50 (backward) \\
Blade thickness impeller (mm) & 0.762 \\
Diffuser inlet radius (mm) & 18.5 \\
Diffuser outlet radius (mm) & 26 \\
\hline
\end{tabular}

Therefore, the geometrical data were given as input to the model, in conjunction with the thermodynamic properties at the compressor inlet, as presented in Table 7.2. The inlet total temperature for the current study was given slightly $(0.6 \%)$, in order to meet the static temperature derived by the thermodynamic analysis that preceded. The developed model estimates the pressure at the outlet of each compressor's element, resulting in a total pressure outlet $14.15 \mathrm{MPa}$, presented a relative error of $1.2 \%$ in comparison with the Model A.

Observing the deviation between the Model A and the own model results, it can be observed that the used relationships composing the developed model can predict sufficiently the compressor performance for the design point. Thus, the same equations are used in a different way, using the inlet and outlet conditions of the compressor, as they have been derived for the cycle under investigation, in order to define the compressor geometry for the given conditions.

Table 7.2. Current model results validation with Model A operating conditions.

\begin{tabular}{lccc}
\hline \multicolumn{1}{c}{ Operating conditions } & $\begin{array}{c}\text { Model A } \\
{[\mathbf{2 8}]}\end{array}$ & $\begin{array}{c}\text { Current } \\
\text { model }\end{array}$ & $\begin{array}{c}\text { Deviation } \\
(\boldsymbol{\%})\end{array}$ \\
\hline Mass flow (kg/s) & 3.53 & 3.53 & - \\
Rotational speed (rpm) & 75000 & 75000 & - \\
Inlet total temperature $(\mathrm{K})$ & 305.5 & 305.5 & - \\
Outlet total temperature $(\mathrm{K})$ & 324.6 & 331.2 & 1.9 \\
Inlet total pressure $(\mathrm{MPa})$ & 7.69 & 7.69 & - \\
Outlet total pressure $(\mathrm{MPa})$ & 13.98 & 14.15 & 1.2 \\
\hline
\end{tabular}




\subsection{Inverse Problem}

The solution of the inverse problem concluded to a final geometry of the centrifugal compressor in order to meet the thermodynamic properties at the compressor outlet. The geometrical characteristic and the velocity triangles were estimated at three levels of the blade span, at hub, mean and tip of the blade. The geometry was derived beginning with the preliminary design presented in Chapter 5.2.1, based on the isentropic enthalpy rise for the compression stage of the considered thermodynamic cycle and the Balje diagram (Figure 2.8). The design procedure presented in 5.2.2 used for the compressor design, employing the Equations (5-2)-(5-40). Table 7.4 summarizes the most significant results of the geometry for the compressor's inducer and impeller, while Table 7.5 concentrates the key geometrical characteristics estimated for the vaned diffuser.

Table 7.3. Results for thermodynamic conditions at inlet and outlet of impeller.

\begin{tabular}{lcc}
\hline & Impeller inlet & Impeller outlet \\
\hline Total pressure, $P_{O}(\mathrm{MPa})$ & 8.61 & 21.51 \\
Static pressure, $P(\mathrm{MPa})$ & 7.8 & 17.03 \\
Total temperature, $T_{O}(K)$ & 305.7 & 342 \\
Static temperature, $T(K)$ & 305.5 & 323 \\
\hline
\end{tabular}

Table 7.4. Geometry of the designed impeller.

\begin{tabular}{lc}
\hline \multicolumn{2}{c}{ Inducer Geometry } \\
\hline Inducer radius at hub (mm) & 144.7 \\
Inducer radius at mean (mm) & 172.3 \\
Inducer radius at tip (mm) & 200 \\
\hline \multicolumn{2}{c}{ Impeller Geometry } \\
\hline Number of full blades & 16 \\
Inlet radius at hub (mm) & 80 \\
Inlet radius at tip (mm) & 200 \\
Impeller outlet radius (mm) & 392 \\
Blade angle of the impeller leading edge at hub (deg) & 46.25 \\
Blade angle of the impeller leading edge at mean (deg) & 61.32 \\
Blade angle of the impeller leading edge at tip (deg) & 69.05 \\
Blade angle of the impeller trailing edge (deg) & 69.75 \\
Axial length (mm) & 160 \\
Blade thickness (mm) & 6 \\
Blade height at leading edge (mm) & 19.6 \\
Gap clearance at tip (mm) & 0.196 \\
\hline
\end{tabular}


Table 7.5. Geometry of the designed diffuser.

\section{Diffuser Geometry}

\begin{tabular}{lc} 
Number of vanes & 25 \\
Diffuser inlet radius (mm) & 462.7 \\
Diffuser outlet radius (mm) & 623.7 \\
Blade angle at diffuser inlet (deg) & 34.9 \\
Blade angle at diffuser outlet (deg) & 44 \\
\hline
\end{tabular}

Table 7.6. Design characteristics comparison of centrifugal compressors for s- $\mathrm{CO}_{2}$ cycle.

\begin{tabular}{lcccc}
\hline & $\begin{array}{c}\text { Mass flow } \\
\mathbf{k g} / \mathbf{s}\end{array}$ & $\begin{array}{c}\text { Speed } \\
\mathbf{r p m}\end{array}$ & $\boldsymbol{\Phi}$ & $\boldsymbol{\Psi}$ \\
\hline SNL & 3.53 & 75000 & 0.029 & 0.58 \\
TITech & 1.2 & 100000 & 0.018 & 0.65 \\
Current design & 1908 & 3600 & 0.031 & 0.54 \\
Turbocharger KKK & 0.2 & 59000 & 0.030 & 0.74 \\
\hline
\end{tabular}

Moreover, Table 7.6 compares the values of mass flow, rotational speed and the flow and stage coefficients derived by the current design model with the centrifugal compressors operating in SNL and TITech test facilities and with a conventional KKK turbocharger used for trucks developed by Steyr-Daimler-Puch. Although the size and mass flow rate present huge variation, the flow and stage coefficients present comparable values, showing that the compressor design meets typical compressors' operation range.

Furthermore, the aerodynamic characteristics for the designed impeller are:

Table 7.7. Results of aerodynamic design.

\begin{tabular}{cc}
\hline Parameters & Values \\
\hline Slip factor & 0.87 \\
Inlet total enthalpy, $h_{01}(\mathrm{~kJ} / \mathrm{kg})$ & 300.2 \\
$\Delta h_{o, \text { Euler }}(\mathrm{kJ} / \mathrm{kg})$ & 31.5 \\
$\Delta h_{o, \text { internal }}(\mathrm{kJ} / \mathrm{kg})$ & 3.1 \\
Exit total enthalpy, $h_{02, i d}(\mathrm{~kJ} / \mathrm{kg})$ & 332.3 \\
$\Delta h_{o, \text { parasitic }}(\mathrm{kJ} / \mathrm{kg})$ & 1.4 \\
Total-to-total aerodynamic efficiency & 0.9 \\
Total-to-total design efficiency & 0.85 \\
\hline
\end{tabular}


The share of the individual loss models at the design point is presented in Figure 7.2. It can be observed that incidence and aerodynamic-blade loading losses are the most significant for the impeller. Moreover the internal losses consist the $70 \%$ of the total losses, while the leakage losses are the major parasitic loss. In the following graph the losses with a radius displacement represent the parasitic losses.

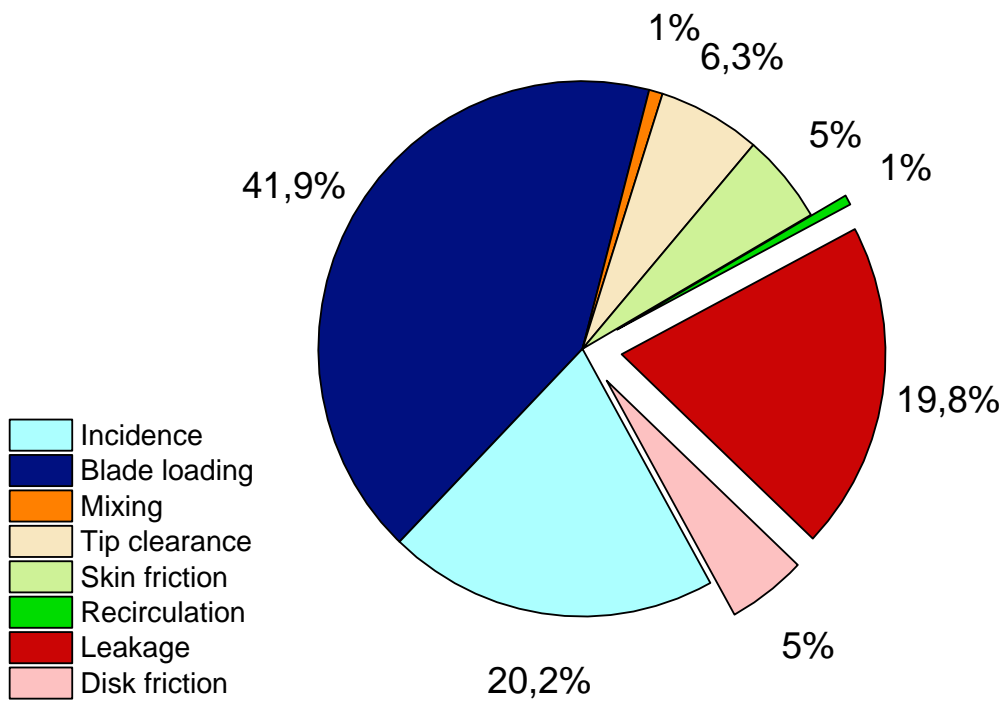

Figure 7.2. Share of the enthalpy losses of impeller.

\subsection{Numerical Solution}

\subsubsection{Real gas effects}

Interpolation is used for calculation of density for each look-up table employed for s- $\mathrm{CO}_{2}$ properties. A difference between interpolated densities for look-up table with varying resolution, leads to differences of mass flow rates and in turn in different velocities at the impeller inlet, even with constant boundary conditions. By calculating different velocities at inlet, Mach numbers also vary and that results in a density distribution that will vary with the table resolution. Figure 7.3 and Figure 7.4 present the changes between the solution for the Mach number and density respectively, for many (500 points) and fewer (100 points) around the critical point. The difference at the pattern of the contour for Mach in the suction side of the blades is obvious, but also there is a difference of the density at the leading edge and at the pressure side between main and splitter blade. This 
difference between the flow solution using two look-up tables with different resolution, leads to the conclusion that the higher resolution look-up table will be used for the next analyses, in order to predict more accurately the flow behavior.

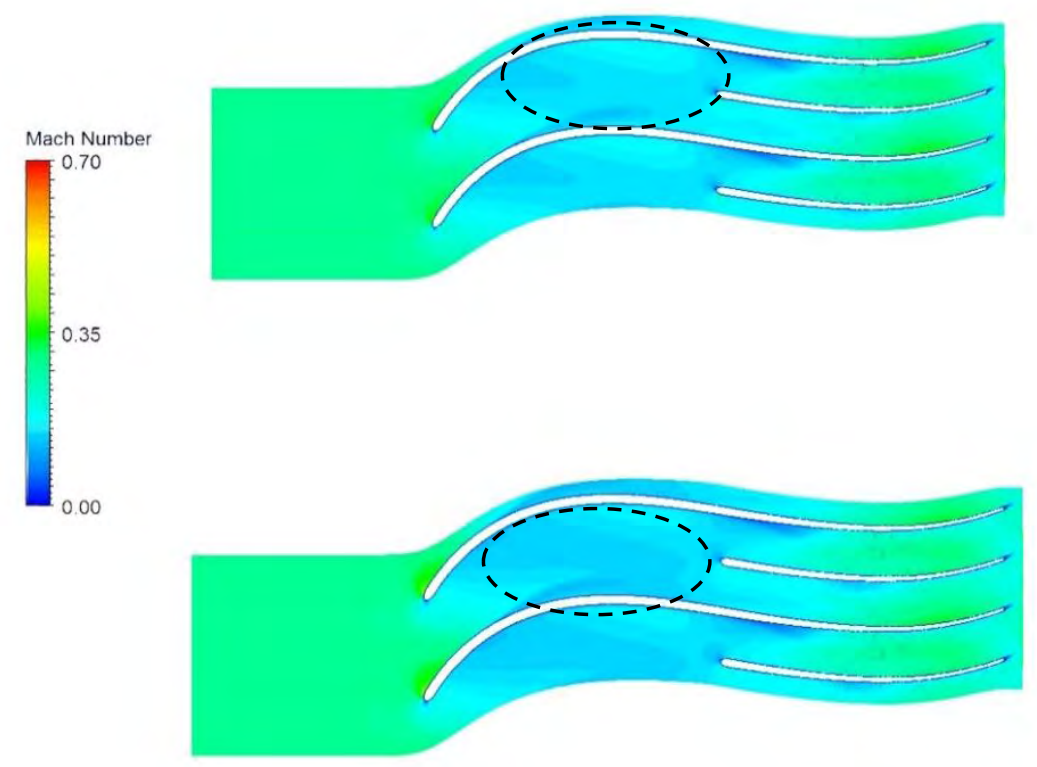

(a)

Figure 7.3. Blade to blade view of Mach number for RGP table with (a) 500 points and (b) 100 points resolution around the critical point.

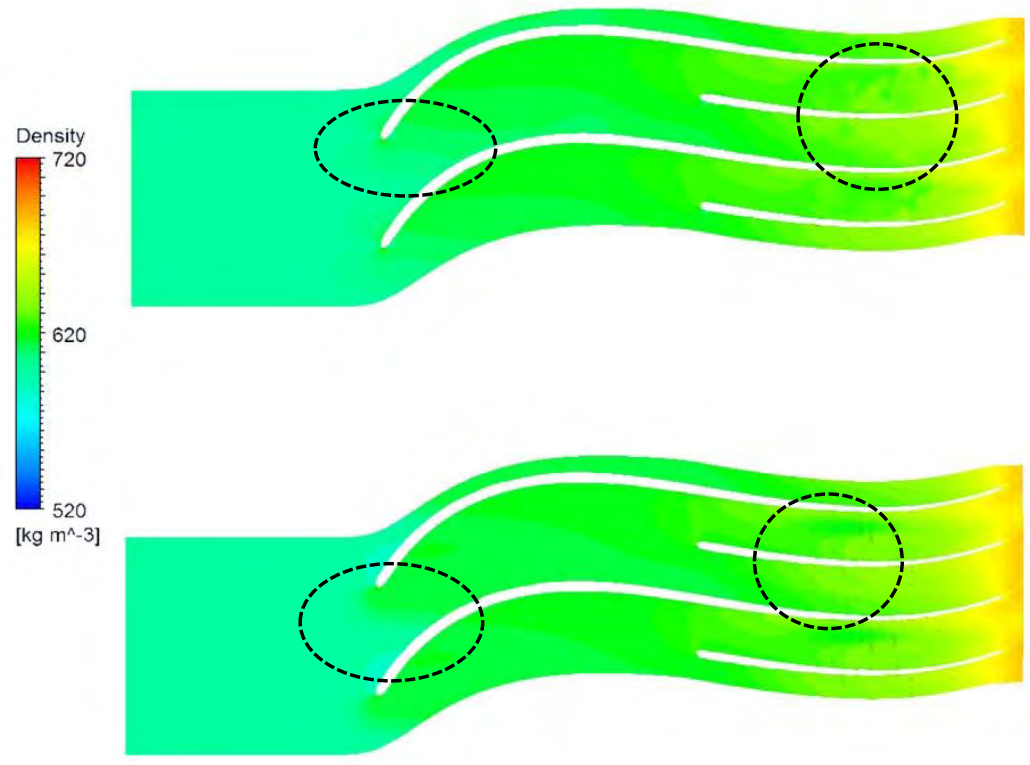

(a)

Figure 7.4. Blade to blade view of density for RGP table with (a) 500 points and (b) 100 points resolution around the critical point. 
Therefore, the further numerical analysis is based on the look-up table with 500 points around the critical point and 100 points far from the pseudo-critical region.

At high flow coefficients, the impeller is subjected to large negative incidence at the leading edge as the stagnation point moves toward the blade suction side. The resulting local flow acceleration causes a decrease in the static pressure and temperature of the fluid. For a real gas, the fluid state eventually falls below the saturation line, suggesting the possibility of condensation in the compressor blade passage. Approaching the critical point increases the possibility of two-phase flow in addition to the larger variations in thermodynamic properties. These effects are studied independently through CFD simulations of the $\mathrm{CO}_{2}$ compressor stage.

The scope of the analysis is to investigate the condensation on the leading edge and the effect of the look-up table resolution on the condensation. Figure 7.5 presents the pressure at the suction side of the impeller, for 500 points and 100 points around the critical point respectively. Therefore, the pressure range on the blade was chosen from 5 to $7.4 \mathrm{MPa}$ as any pressure above this value is far from condensation and in the figures are illustrated only the regions with pressure below the condensation pressure. From these two figures it is observed that the pressure contours at the same regions present variations, indicating that the changes in pressure can be captured more efficiently with the high-resolution look-up table.

Hence, the number of the points in the region around the critical point can affect also the condensation effect on the blade's leading edge. In following sections the condensation effects will be discussed further, taking into account the look-up table with higher resolution.

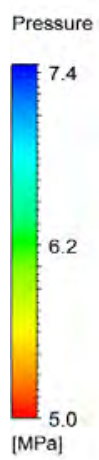

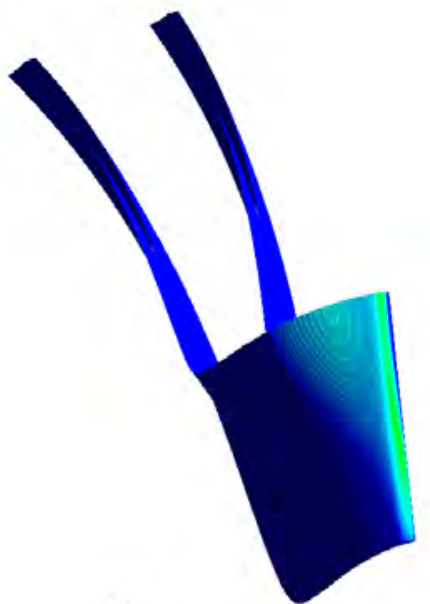

(a)

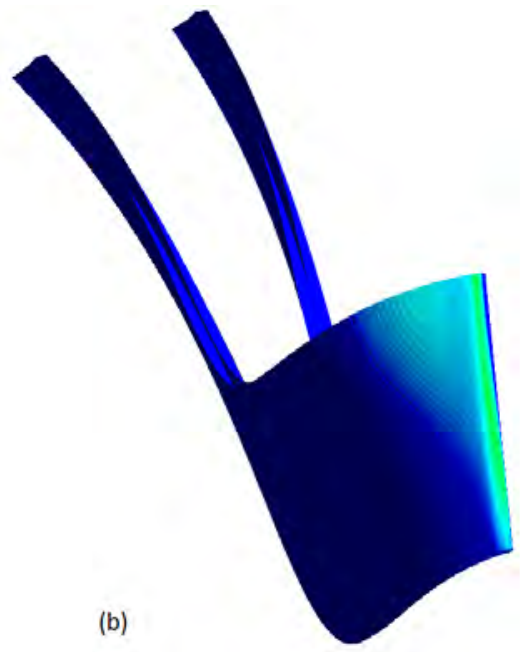

Figure 7.5. View of one blade from inlet for condensation investigation with table resolution of (a) 500 and (b) 100 points. 


\subsubsection{Aerodynamic analysis for design point}

The numerical analysis resulted in the aerodynamic characteristics of the flow for the design point. First of all a comparison between the results of the meanline analysis by the developed tool and the CFD results is presented. The CFD analysis presents good agreement with the 1D model, with a maximum of $1.9 \%$ for total temperature at outlet.

Table 7.8. Validation between 1D meanline and CFD results.

\begin{tabular}{cccc}
\hline Parameter & 1D model & CFD & Error $(\%)$ \\
\hline Total pressure inlet & 7.6 & 7.6 & - \\
Total pressure outlet & 20 & 19.8 & 1 \\
Total temperature inlet & 305.1 & 305.1 & - \\
Total temperature outlet & 342.1 & 335.6 & 1.9 \\
Density outlet & 691.4 & 678.2 & 0.2 \\
\hline
\end{tabular}

The analysis of the results continues with the observation of various flow fields estimated by the CFD simulations for the impeller. Figure 7.6 and Figure 7.7 show the total pressure and temperature field for the compressor stage at mid-span, for the design point. The flow exits the impeller at a total pressure close to $18 \mathrm{MPa}$ and total temperature of $335 \mathrm{~K}$.

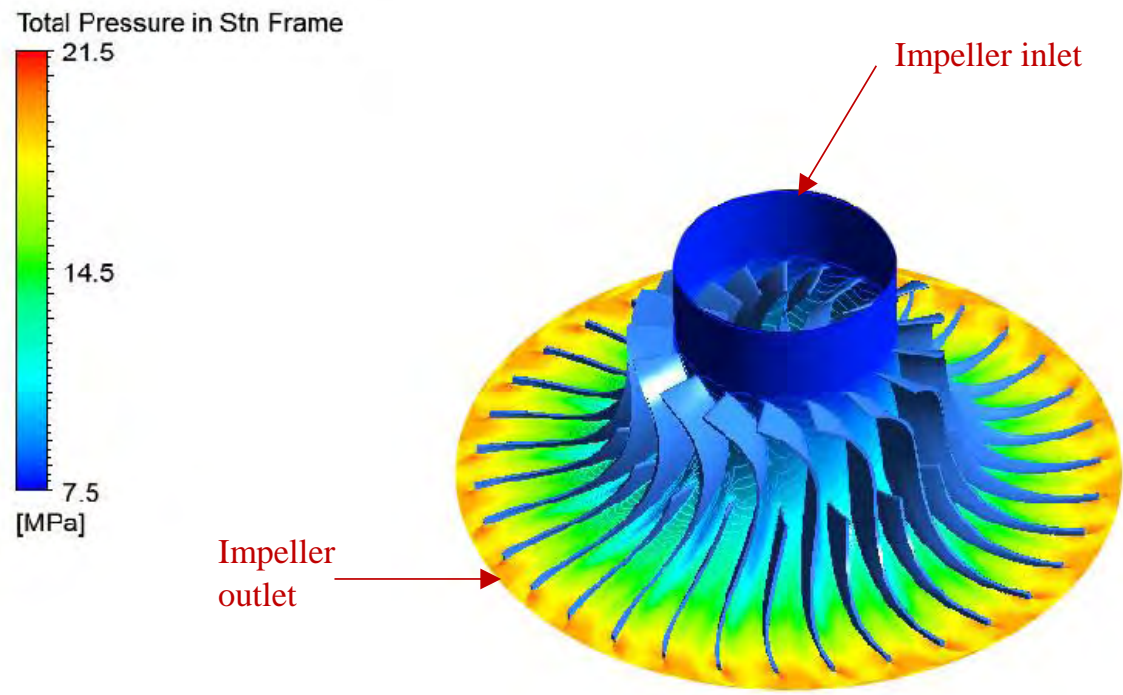

Figure 7.6. Lateral view for total pressure contour for the whole compressor stage at span 50\%. 

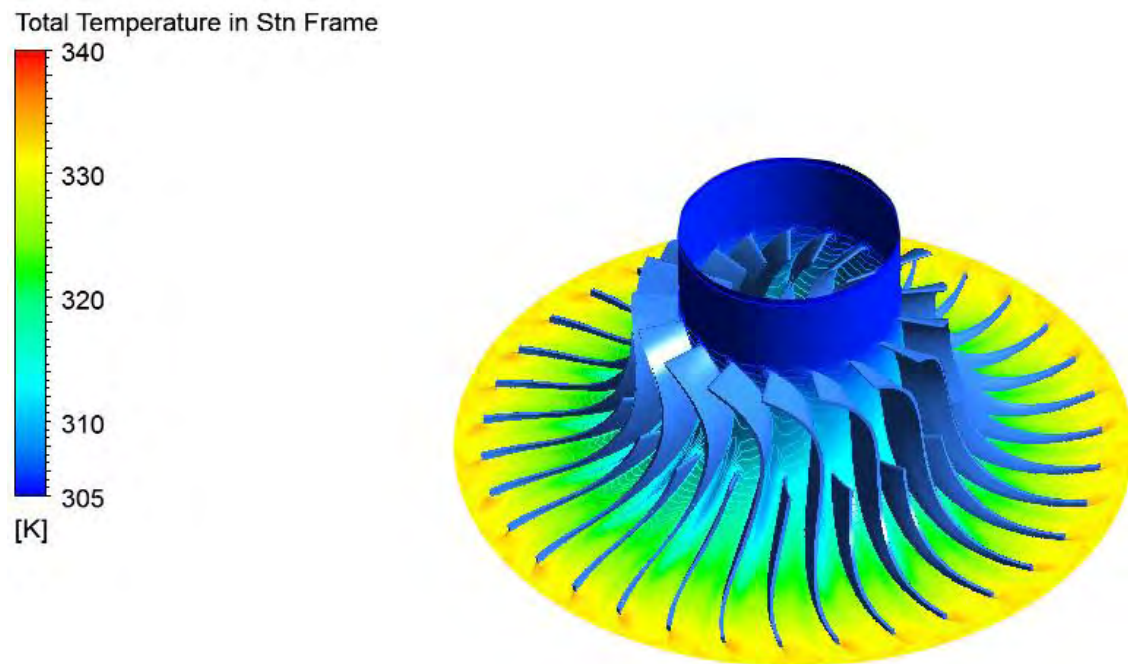

Figure 7.7. Lateral view for total temperature contour for the whole compressor stage at span $50 \%$.

Figure 7.8 presents the velocity flow field for the compressor at span 90\% near the tip clearance gap, where the leakage flow creates more intense recirculation. The figures show separation close to tip because of the high angle of attach at the blade leading edge.
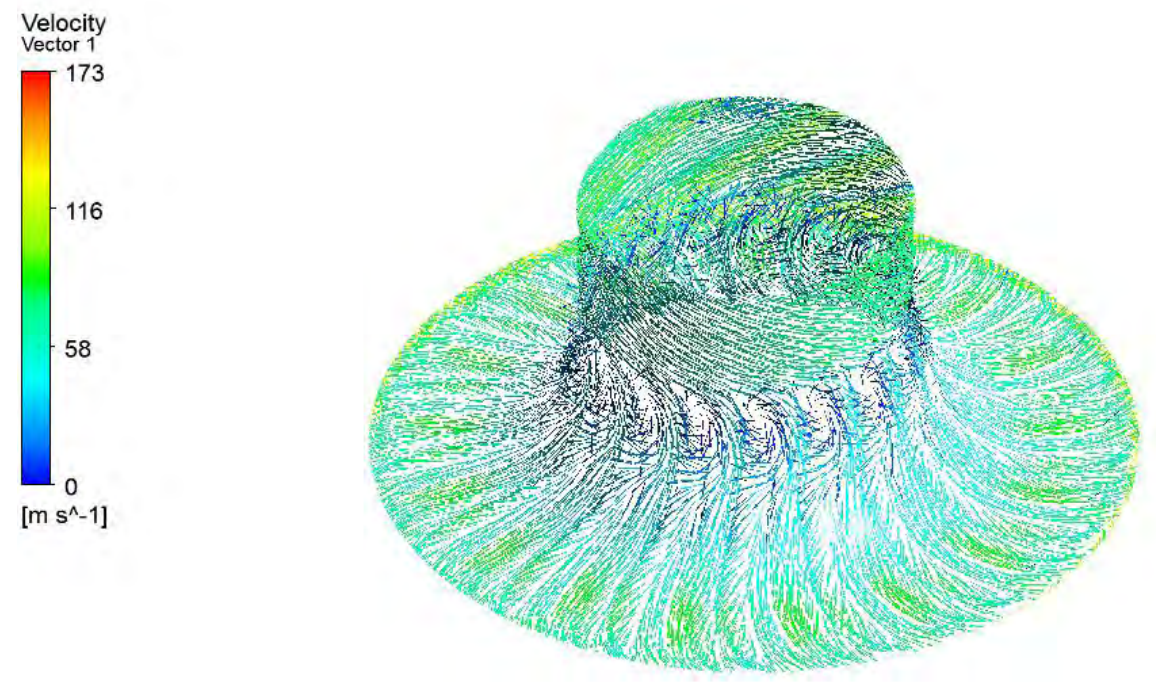

Figure 7.8. Lateral view for velocity vectors for the compressor stage at span $90 \%$.

Figure 7.9 presents the blade to blade view of total pressure and total temperature, where the changes are obvious close to main and splitter blade throat. 
Figure 7.10 presents the blade to blade view of the Mach number of relative and absolute reference frame respectively.
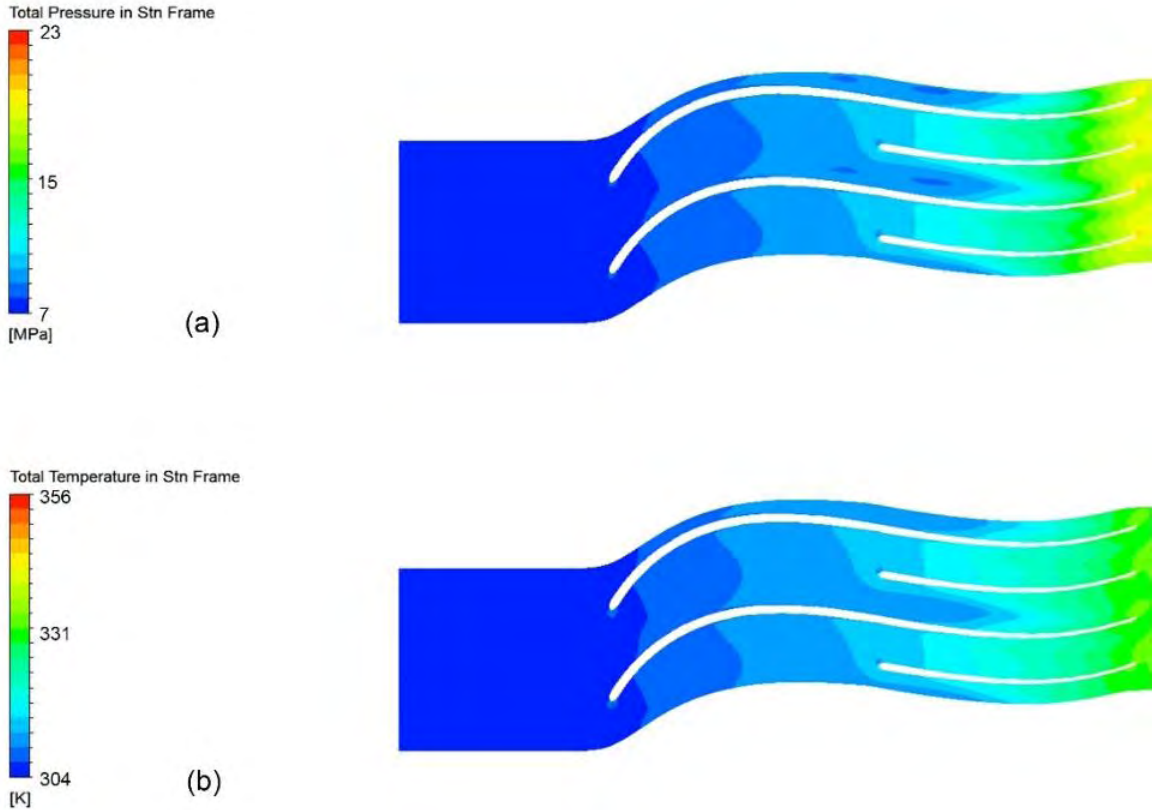

Figure 7.9. Blade to blade view of (a) total pressure, (b) total temperature.
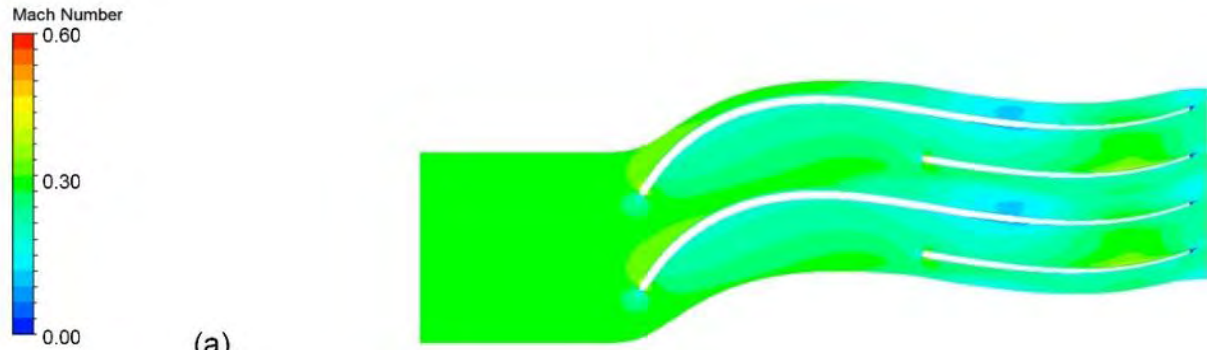

(a)
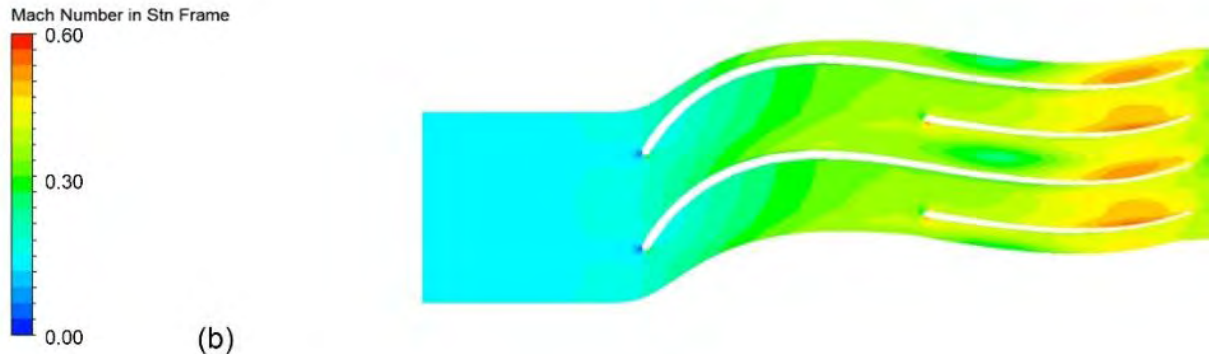

(b)

Figure 7.10. Blade to blade view of (a) relative Mach number, (b) absolute Mach number. 


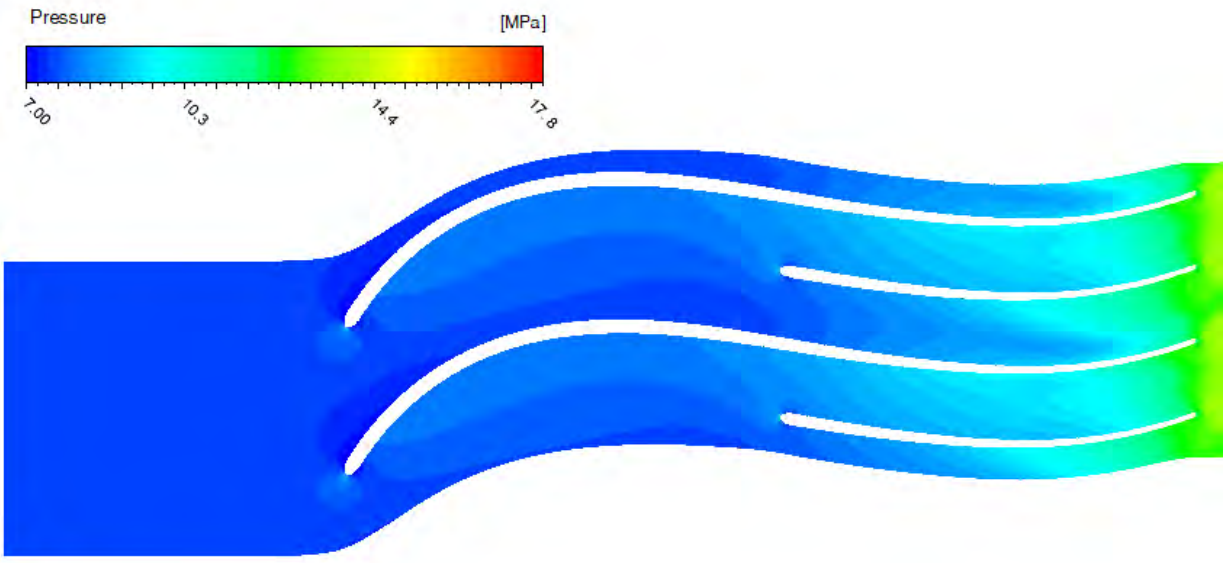

Figure 7.11. Blade to blade view of static pressure at mid-span.

Figure 7.11 presents the static pressure distribution at the blade to blade view for the impeller. Moreover, the relative Mach number for various planes along the compressor is presented in Figure 7.12, showing the deceleration of the relative velocity component.

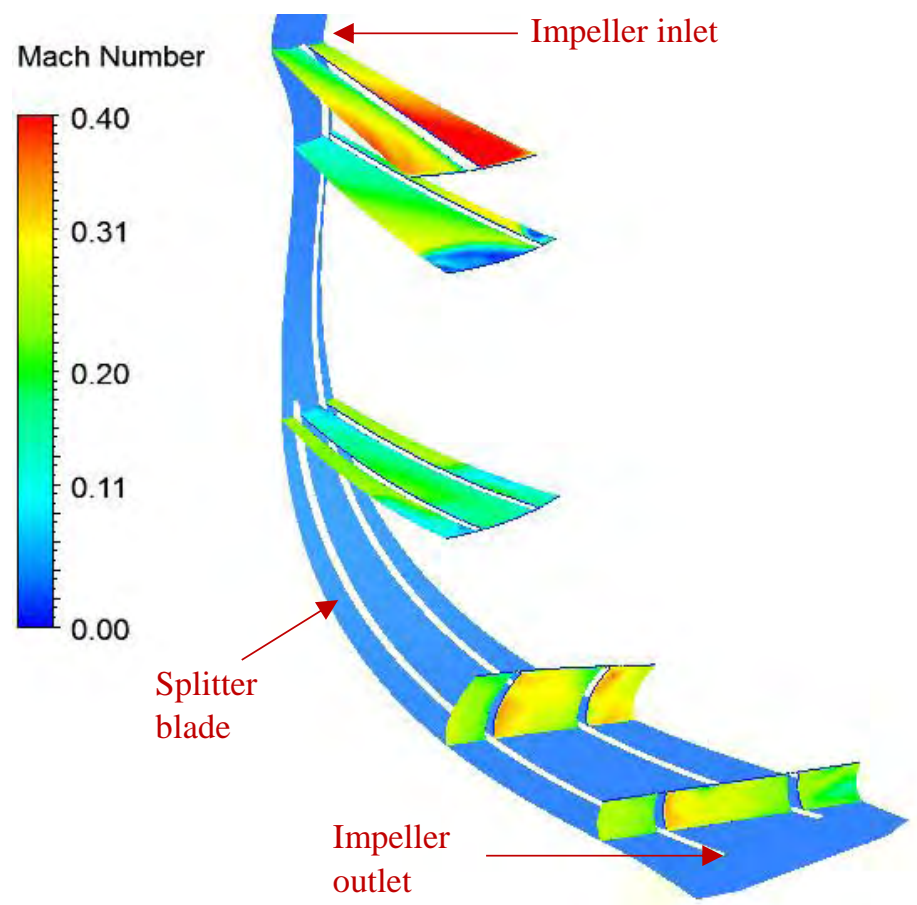

Figure 7.12. Relative Mach number along the impeller passage. 
It is observed how the flow accelerates at the inlet intensely and then how the Mach number reduces along the passage. The Mach number seems to present relatively low values at every plane, which is desirable in the compressor design for the considered power cycle, in order to avoid pressure drop in the subcritical region. Moreover, the relative velocity variation is presented in Figure 7.13 in order to give the order of magnitude of the velocity that flow the impeller.

Besides, Figure 7.14 presents the vectors of the velocity at span $99 \%$ of the blade. It can be seen that there is a secondary flow that follows the direction from outlet to inducer of the impeller, while the high curvature of the inducer creates also regions where the velocity is zero.

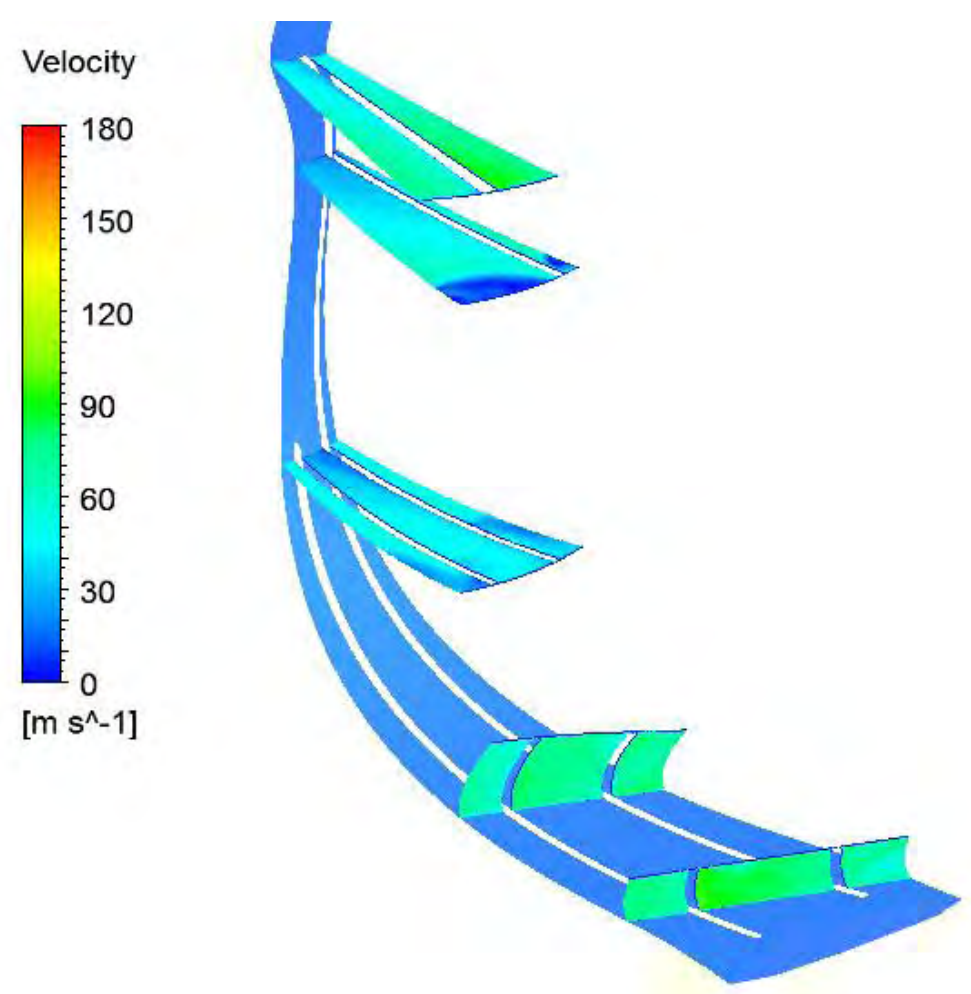

Figure 7.13. Relative velocity variation along the compressor. 


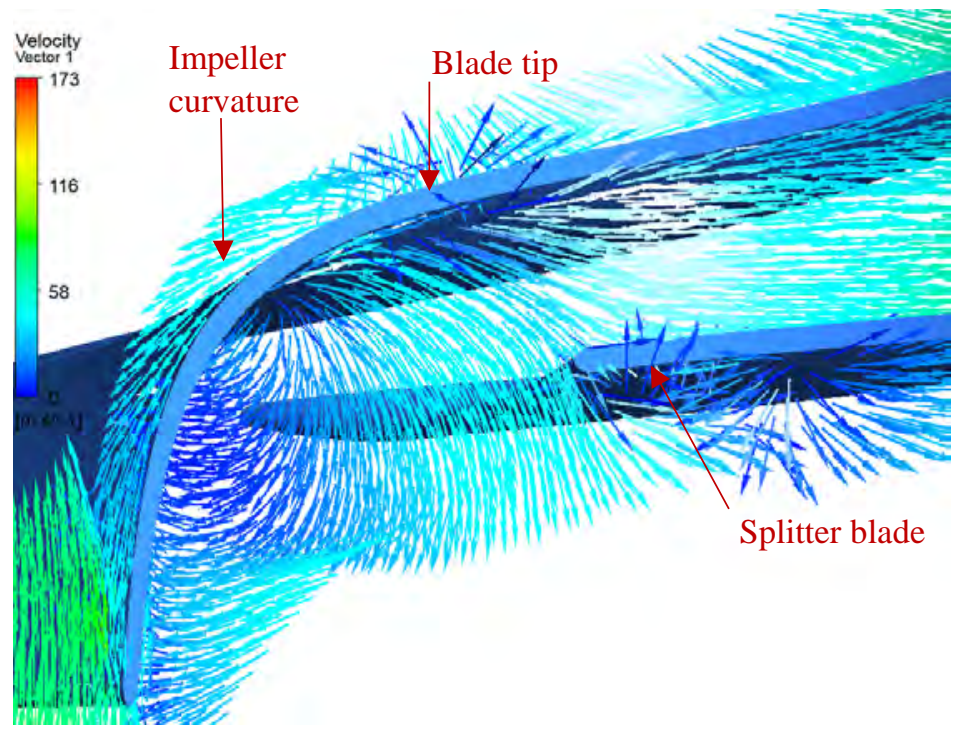

Figure 7.14. Leakage flow at impeller tip.

Figure 7.15 presents the span-wise variation of static pressure showing that the upper part of the blade is possible to present condensation problem.

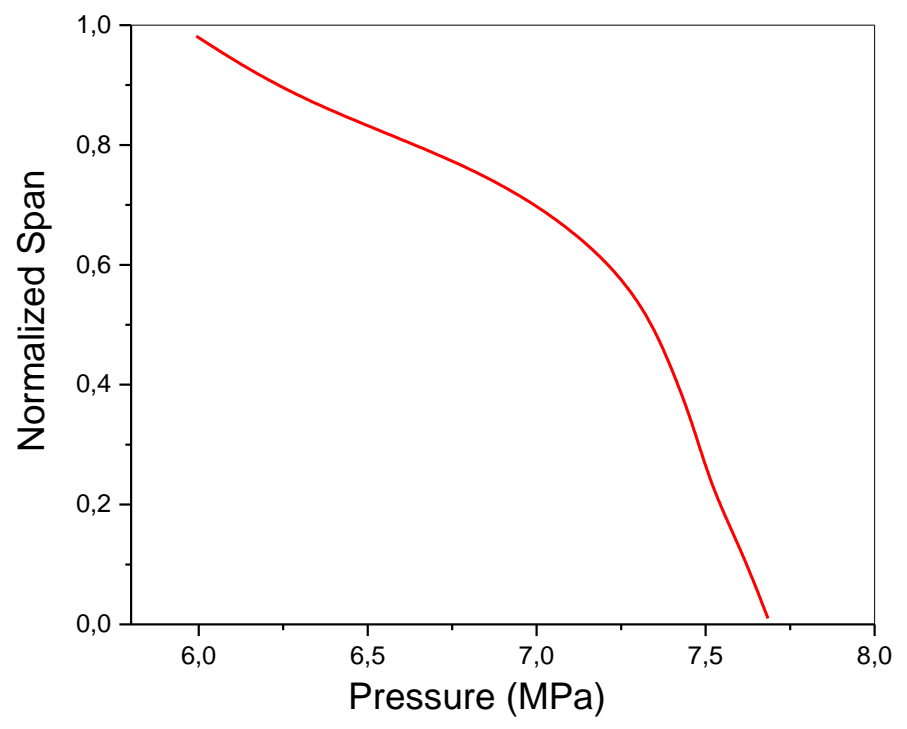

Figure 7.15. Span-wise variation of static pressure close to throat.

Contours of dimensionless axial-wise vorticity at the inlet plane is presented in Figure 7.16. Dimensionless stream-wise vorticity is calculated using the following equation: 


$$
\Omega_{z}=\left(\frac{\partial V}{\partial X}-\frac{\partial U}{\partial Y}\right) \frac{L_{p}}{V_{\text {inlet }}}
$$

where $V$ is the span-wise velocity, $U$ the pitch-wise velocity and $L_{p}$ the blade pitch. It can be seen how the vorticity varies from pressure to suction side and mainly the intense vorticity as the flow turns passage.

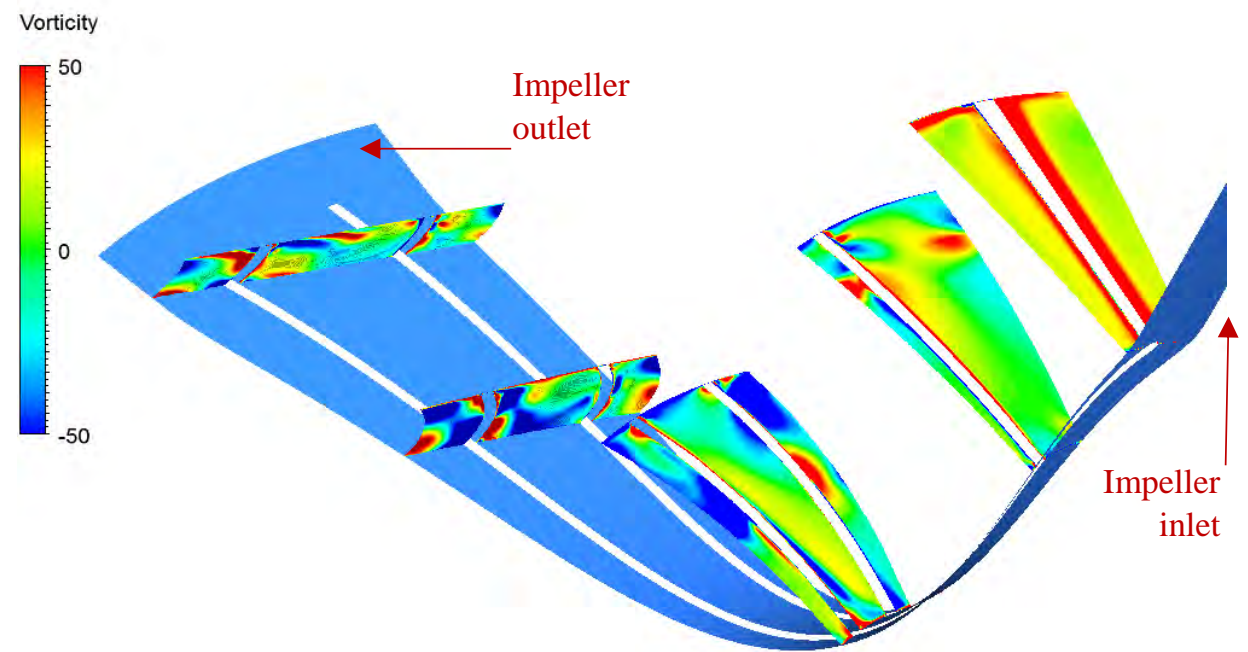

Figure 7.16. Contours of vorticity along the designed impeller.
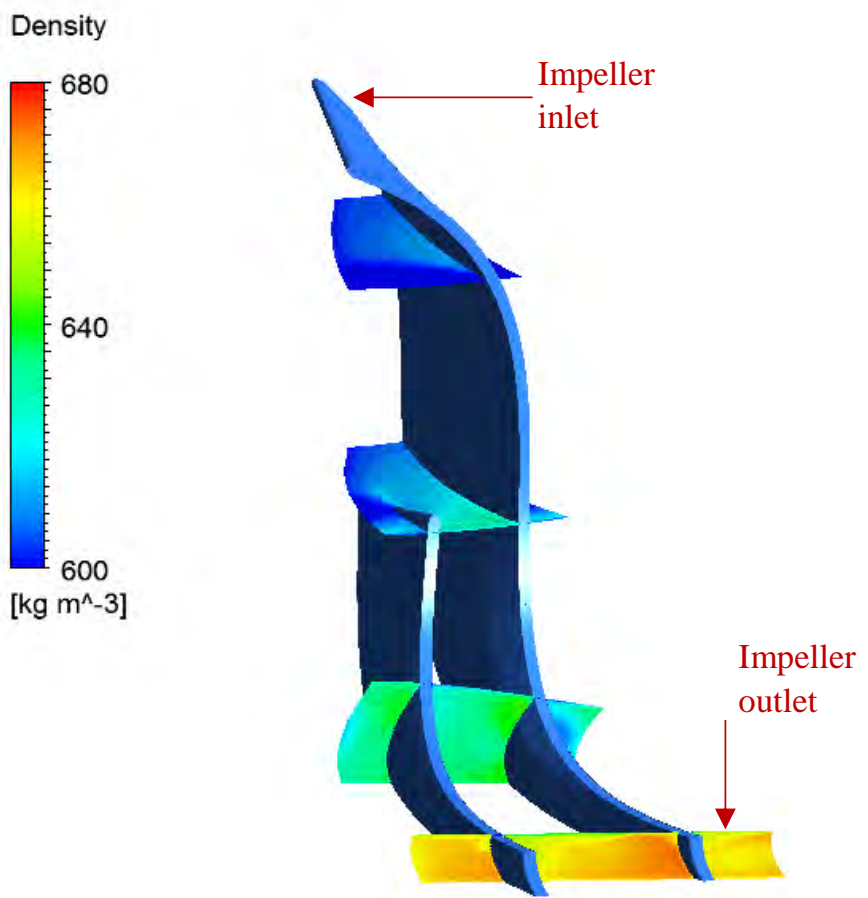

Figure 7.17. Contours of density at various planes along the impeller. 
Contours of density at various planes along the impeller are presented in Figure 7.17. It can be observed that the density increases along the impeller, while it is shown that the wake invokes a smaller density at the planes, in comparison to the flow-jet. The maximum density is presented at the outlet plane as expected, while the outlet density has a value about $670 \mathrm{~kg} / \mathrm{m}^{3}$ at the impeller pressure side.

Furthermore, the performance of the centrifugal compressor was investigated for a range that the compression system may operate in order to evaluate the offdesign operation of the compressor. Off-design performance of the turbomachinery will influence the part-load efficiency, operation strategy and limits for the cycle. Figure 7.18 demonstrates the pressure ratio variation with mass flow for each rotational speed, while the respective efficiency variation is presented in Figure 7.19 .

A total of 48 simulations was used to retrieve the compressor map. Six rotational speeds of the impeller were considered: 2700 (75\%), 3060 (85\%), 3240 (90\%), $3420(95 \%), 3600(100 \%)$ and $3960(110 \%)$ rpm. For each of these angular velocities, a sequence of 8 simulations whose mass flow outlet boundary condition is incremented gradually from $800 \mathrm{~kg} / \mathrm{s}$ to $2600 \mathrm{~kg} / \mathrm{s}$ has been utilized to define each speed line. More operating points were simulated, but because of instabilities of the simulations, the results were ignored and not included. Initialization was performed by means of a steady-state simulation at conditions close to those of design. The setup of the various boundary conditions and the definition of all 48 simulations has been facilitated by the use of the "Parameters set" window of Ansys Workbench.

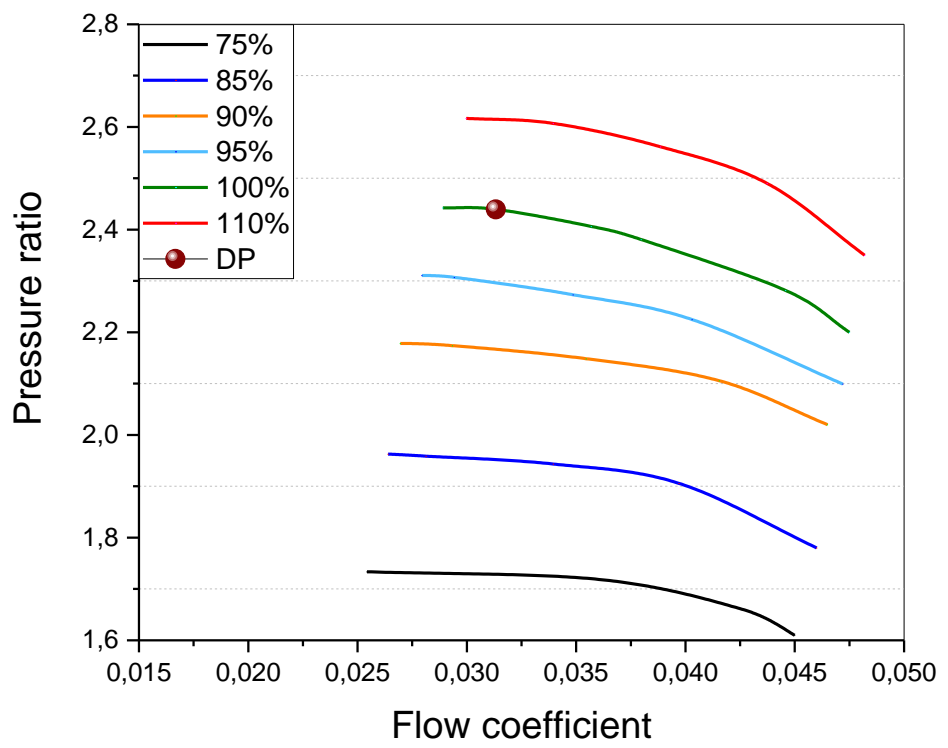

Figure 7.18. Compressor performance map for various rotational speeds. 


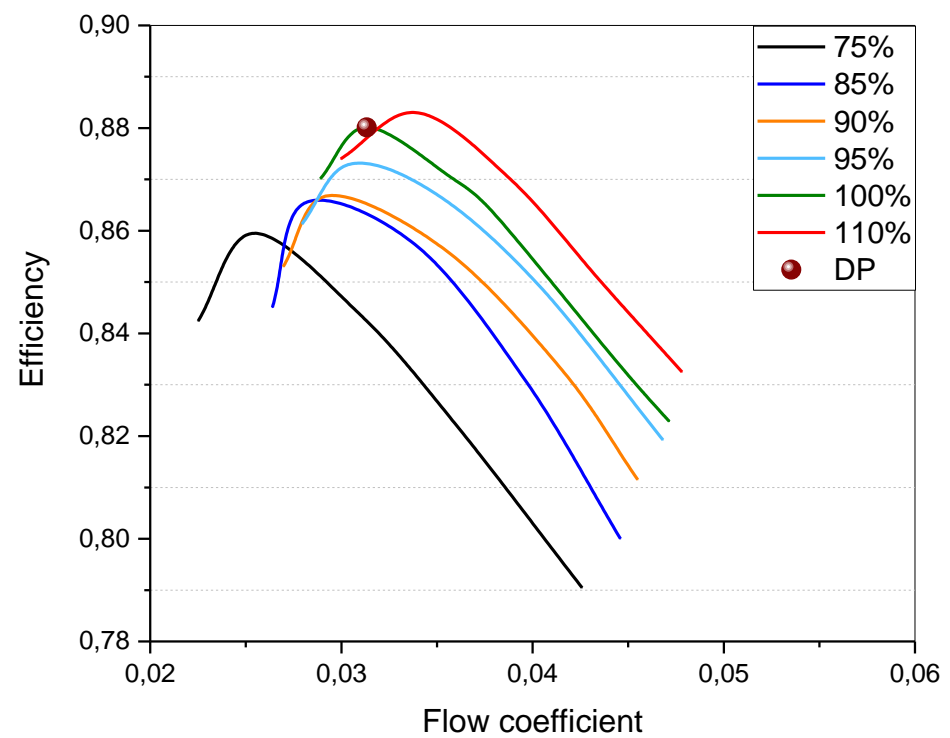

Figure 7.19. Efficiency-flow coefficient for varying rotational speed.

The curves show a wide mass flow rate operating range at various shaft speeds. The pressure ratio curves present lower values at low and high mass flows, while the efficiency presents a steep reduction moving to the surge and choke lines. The established criterion that was applied for the surge limit was $d p / d \dot{m} \geq 0$. Many of the curves show that the phenomena like choke possibly occur, such as abrupt drop of efficiency, although the actual limits were not reached because of the difficulty of converging the s- $\mathrm{CO}_{2}$ compressor near performance failure in the CFD model. Moreover, choking mass flow can be calculated from the continuity equation with throat area, critical sonic velocity, density in the throat and blockage factor at the inlet $\left(\dot{m}_{c h}=A a_{c} \rho_{c}(1-B)\right)$. The speed of sound near $\mathrm{CO}_{2}$ critical point, as in case of impeller inlet conditions, has values at the range of $200-300 \mathrm{~m} / \mathrm{s}$ (Figure 3.2). These velocities are very high and cannot be achieved even for high mass flow rates for the designed inlet passage, so the choke limit cannot be easily reached and it is not presented in the performance map. It can be observed that the design point is located close to the surge line. However, the mass flow rate increase would lead to inlet velocity increase, which would lead to lower pressures at leading edge. Therefore, the possibility of condensation at the leading edge is investigated. 


\subsubsection{Condensation investigation}

The design of the centrifugal impeller turns the flow in the direction of the blade at the inlet, accelerating the flow and resulting in temperature drop locally at the leading edge and the suction side, which means that a fraction of the flow locally could fall inside the two-phase region and condensation could appear. As the operating inlet conditions change the liquid volume fraction may vary, but it can also depend on the rotational speed and the mass flow rate. The condensation impact on compressor performance is examined at this section. Even for the design point the two-phase effects may not be avoided at the leading edge. The inlet temperature considered for the designed cycle is $305 \mathrm{~K}$, just above the critical temperature $(304.1 \mathrm{~K})$. Figure 7.20 presents the temperature distribution at the impeller's leading edge. It is shown that above span 0.6 the temperature drop is getting more significant reaching a temperature $293 \mathrm{~K}$ close to the tip. The temperature distribution at the impeller's blade correspond to a saturation pressure distribution. The possibility of condensation is assessed examining the supersaturation (ratio of fluid pressure to the equilibrium pressure), presented in Chapter 5, for this study. So, for the specific inlet temperature, the difference from the saturation pressure could lead to outcomes regarding the condensation. When this value is getting positive, the possibility of condensation is getting higher.

Figure 7.21 presents one blade of the designed impeller, showing in blue color the pressure on the blade that is in the supercritical region. It can be seen that the contours presented show the regions of the blade with supersaturation, which possibly leads to two-phase flow. The blade leading edge and especially the region close to tip presents condensation, however this volume fraction is limited and cannot affect significantly the compressor operation. However, as the mass flow increases the flow inlet velocity also increases, resulting in considerable velocity changes at the blade's leading edge and suction side. The increased condensation on the impeller results in a limited operational range for the compressor in order to avoid regions of low pressures at the suction side, especially when the pressure drops significantly below the critical value. 


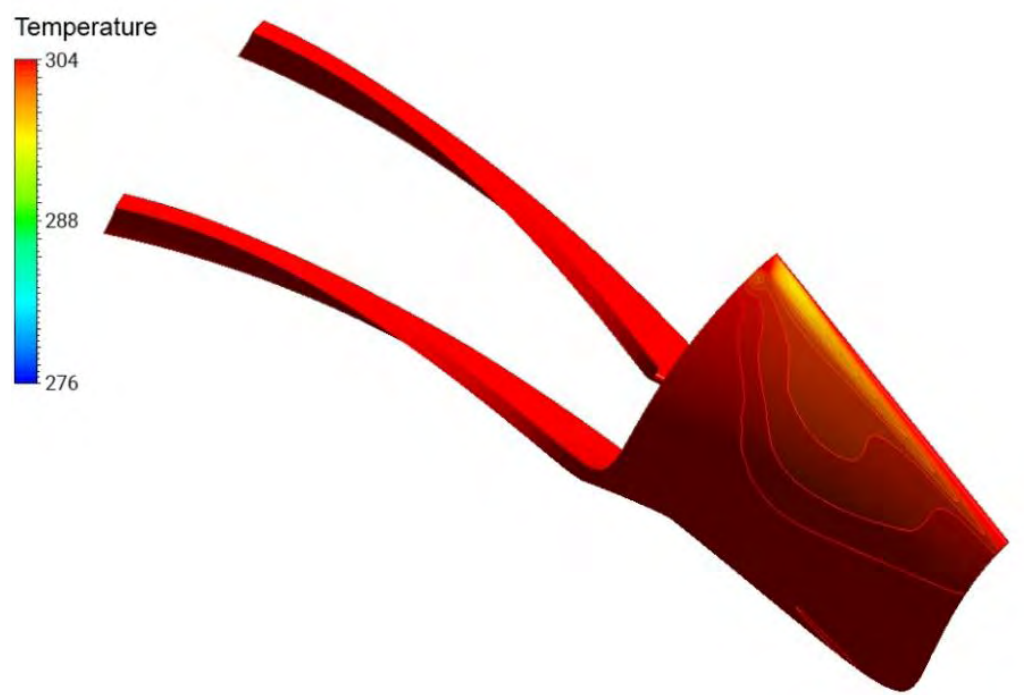

Figure 7.20. View of one blade from inlet indicating the temperature distribution at the impeller inlet $\left(T_{\text {crit }, \mathrm{CO} 2}=304.1 \mathrm{~K}\right)$.

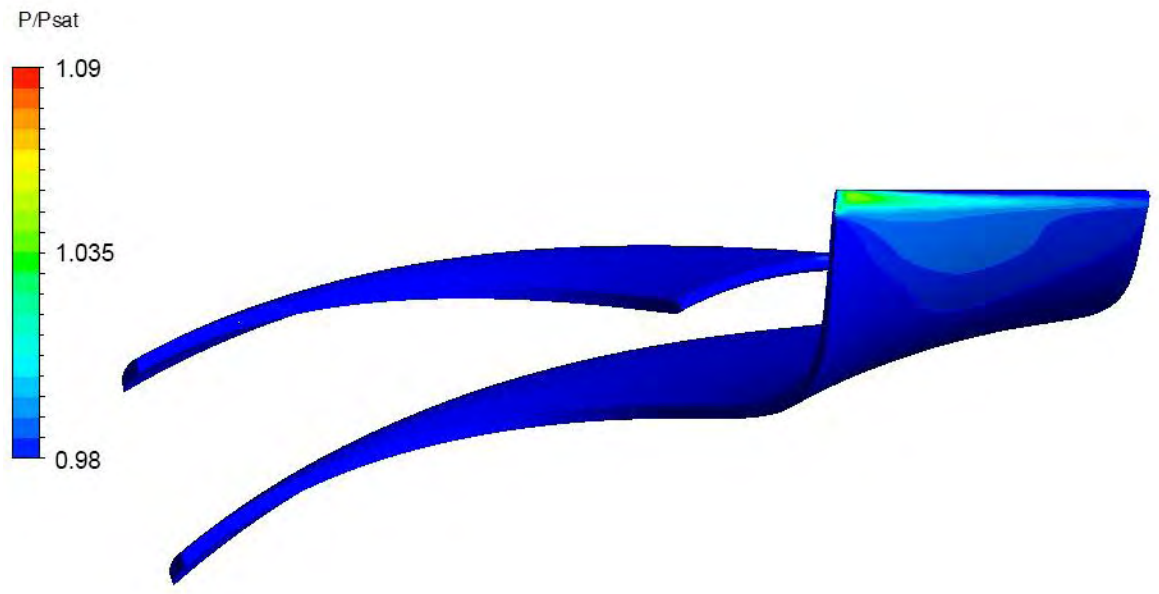

Figure 7.21. View of one blade from inlet investigating condensation on the suction side of the impeller's blade for design point. 


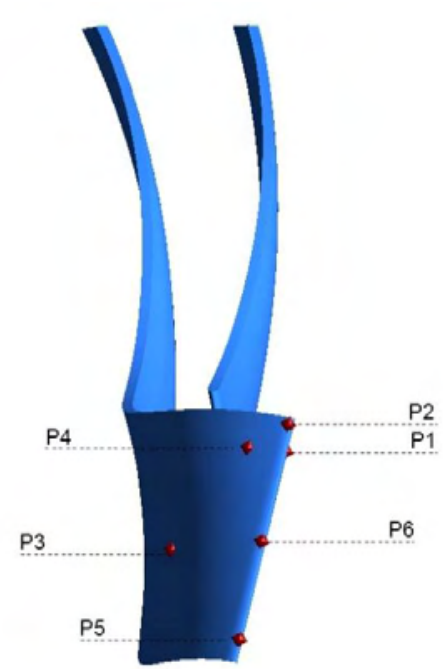

Figure 7.22. View for one blade from inlet indicating the position of the pressure probes on the blade surface.

Moreover, variation of rotational speed leads also to varying pressure regions at the suction surface. For this reason, various pressure probes (points) on the blade surface were selected, in order to examine how the pressure varies at each point for the operational range presented in the performance map of Figure 7.18.

Figure 7.22 presents the pressure probes on the regions where the condensation is possible and the two-phase effects could be significant. Probe 1 is placed at the pressure side, probe 2 at the blade tip at leading edge, probe 3 after the impeller throat, probe 4 close to blade tip and at the region that the flow is accelerated, probe 5 at mid-span of the leading edge and finally, probe 6 is laid close to hub at leading edge.

Figure 7.23 shows the results for the two pressure probes (points) presented in Figure 7.22, which depict the maximum variation in pressure in respect with mass flow variation. The horizontal line consists the critical pressure, as every point existing below this line is in the subcritical region. It can be observed that for many cases in the operational range, the pressure of each point is under the critical line and are compared with the saturation pressure. For every rotational speed, higher mass flow rates lead to condensation at the leading edge close to tip $\left(\mathrm{P}_{2}\right)$ and at the upper region of the suction side $\left(\mathrm{P}_{4}\right)$. This behavior is explained by the high inlet velocity, which is increasing for higher rotational speeds and higher mass flow rates resulting in two-phase flow at the leading edge and the region close to tip of the impeller. On the other hand, the pressure examined at span $50 \%$ close to the throat $\left(\mathrm{P}_{3}\right)$, at span around 5\% $\left(\mathrm{P}_{5}\right)$ and span around 50\% $\left(\mathrm{P}_{6}\right)$ showing that when the mass flow rate increases, no condensation will occur at this region for every rotational speed. 

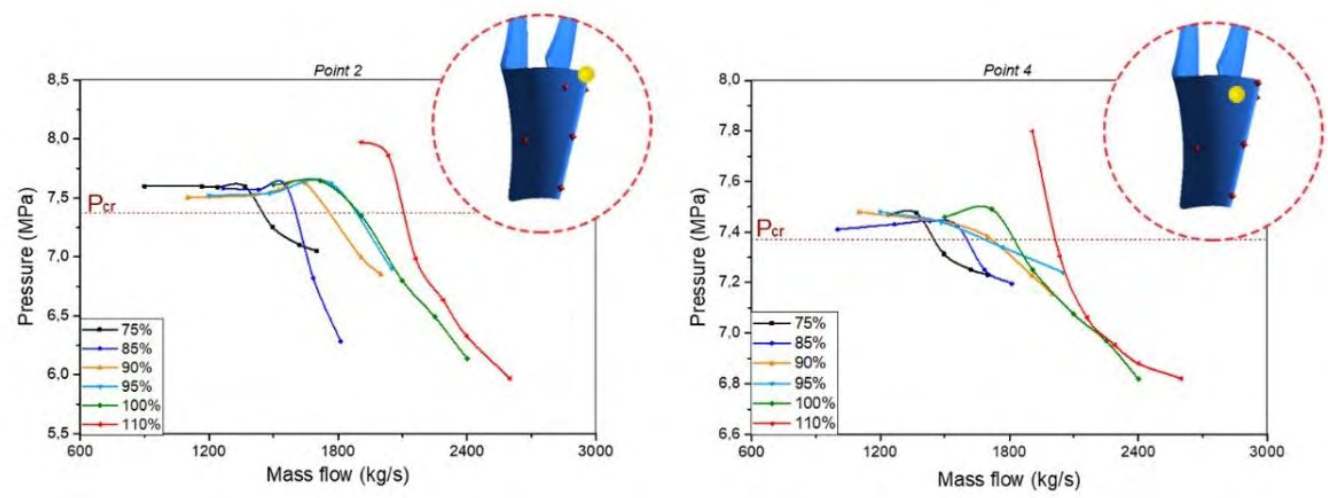

Figure 7.23. Pressure variation for probes at leading edge.

Therefore, the initial performance map presenting the operational range of the compressor has to be limited in a shorter range, where the pressure at the impeller suction side is not below the saturation curve and the two-phase effects do not impact on its performance. The condensation limits because of the velocity changes as the rotational speed and the mass flow vary, invoke a limited compressor operation in order to ensure supercritical flow in the compressor. The condensation limits are a result of the above mentioned analysis that investigates the pressure expansion as the flow accelerates for each point at the impeller blade. Thus, Figure 7.24 presents a performance map for rotational speeds from $75 \%$ to $110 \%$, where the sketched area of the map represents the operational range with condensation risk.

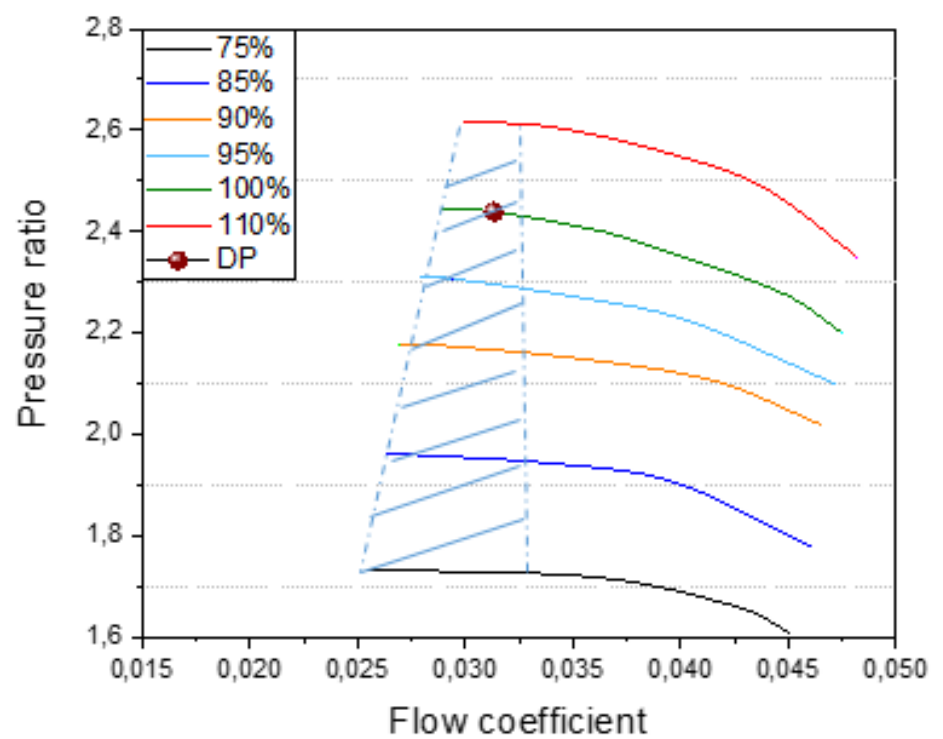

Figure 7.24. Compressor performance map with condensation line-limit. 


\subsubsection{Tip clearance effect on condensation}

The blade tip clearance has a significant impact on the compressor aerodynamic performance, mechanical strength and vibrations. The flow field can vary with the increase of the tip clearance gap and the low pressure regions at the blade tip and suction side can lead to condensation. The leading edge presents high condensation risk, as the surface curvature is higher and the sharp edges accelerate the flow more intensely. As the tip clearance gap increases, the aerodynamic performance is considered to get worse. For each $1 \%$ increase of tip clearance, a $1 \%$ of efficiency reduction is expected. However, since the designed geometry for $\mathrm{s}-\mathrm{CO}_{2}$ compressor has really small dimensions, the $1 \%$ of blade height is only $0.196 \mathrm{~mm}$, which is considered extremely difficult to be achieved. The smallest tip clearance gap that can be constructed in modern turbomachines is $1 \mathrm{~mm}$. Therefore, the aerodynamic performance of an impeller with tip clearance gap that equals $5 \%$ of blade height (about $1 \mathrm{~mm}$ ) was examined, focusing on the pressure distribution and how the tip clearance increase affect the two-phase flow at blade tip, leading edge and suction side. The differences between the two tip clearance gaps are studied by means of CFD.

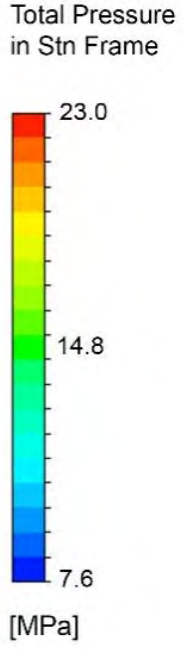

(a)

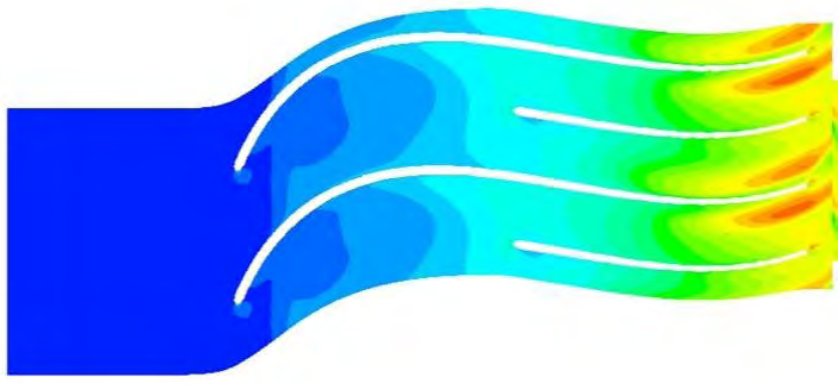

(b)

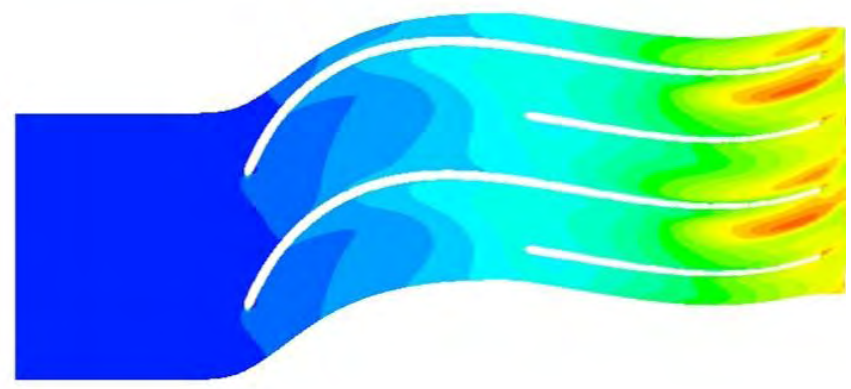

Figure 7.25. Blade to blade view of total pressure at span 0.9 for tip clearance (a) $1 \%$ and (b) $5 \%$ of blade height. 
First of all, the total pressure and velocity distribution are investigated in order to study how the tip clearance affects significantly the flow phenomena and has an important impact on aerodynamic performance of the impeller. The following figures present the variation of characteristic parameters of the flow at mid-span and almost at tip (normalized span 0.9), in order to examine the variations of the flow for each gap. Figure 7.25 presents the variations of total pressure for absolute frame of reference at normalized span 0.9 , showing that for tip gap 5\% of blade height the total pressure starts to increase just at the throat, while for case of $1 \%$ slightly later.

Figure 7.26 presents the blade to blade velocity at mid-span for the two tip clearance cases. The second case seems to present slightly lower velocity values at pressure side, while the recirculation close to exducer is lower in comparison with tip clearance $1 \%$.

In Figure 7.27 the blade to blade static entropy is illustrated at mid-span for the two examined tip clearance gaps, showing the irreversibilities generation along the blade. It can be noticed that for tip gap $1 \%$ the inducer presents a larger region with lower entropy, while after splitter blade the entropy increases faster than the case with tip gap 5\%.

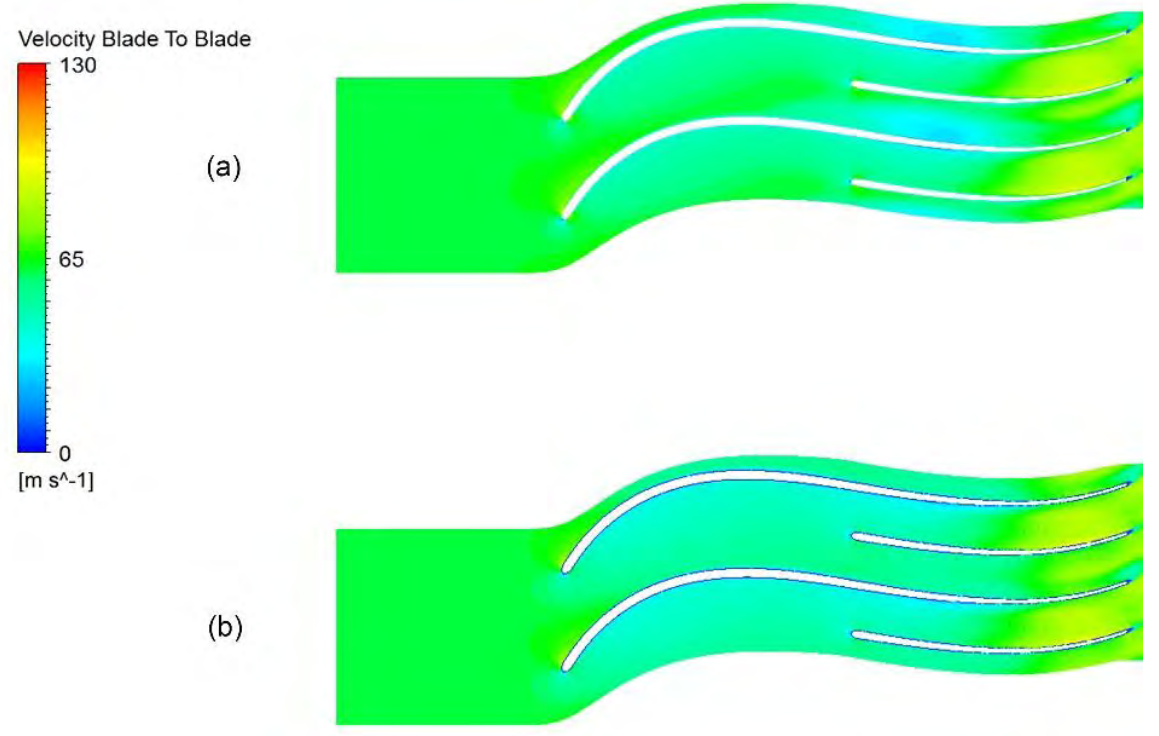

Figure 7.26. Blade to blade velocity for tip clearance (a) $1 \%$ and (b) $5 \%$ of the blade height. 


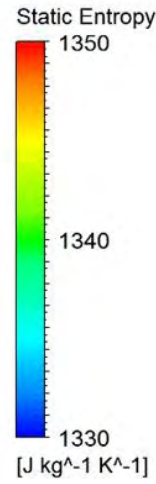

(a)

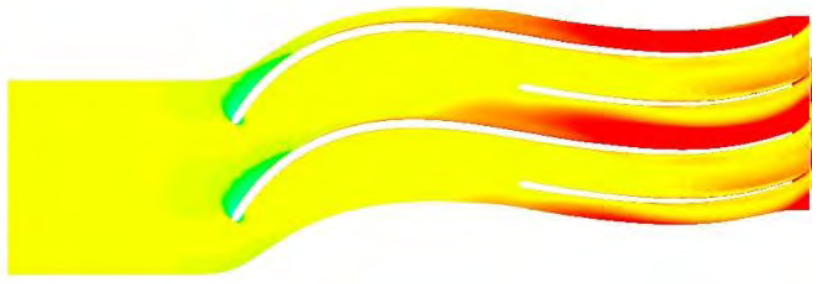

(b)

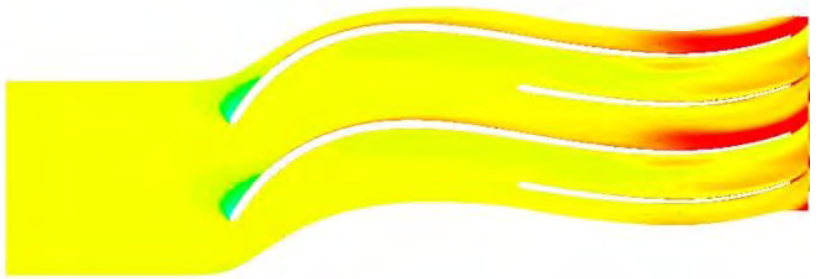

Figure 7.27. Blade to blade entropy distribution for tip gap (a) $1 \%$ and (b) $5 \%$ of blade height.

Furthermore, the dimensionless axial-wise vorticity is estimated at three planes for each tip clearance gap and is presented in Figure 7.28. The planes are placed at normalized chord $0 \%$ (inlet), $15 \%$ and 35\% (after splitter leading edge).

(a)

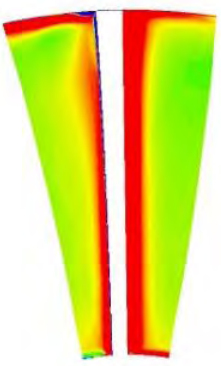

$0 \%$

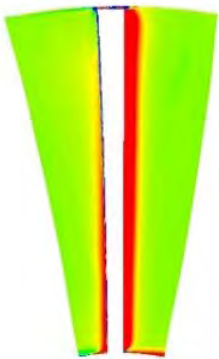

$0 \%$



$15 \%$

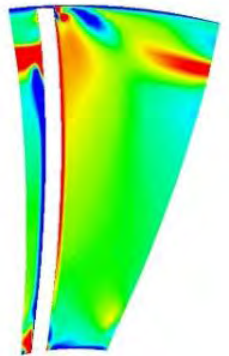

$15 \%$

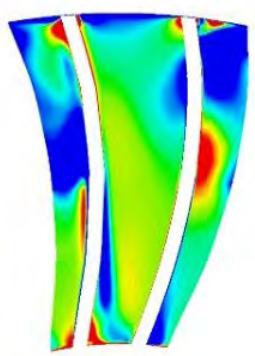

$35 \%$

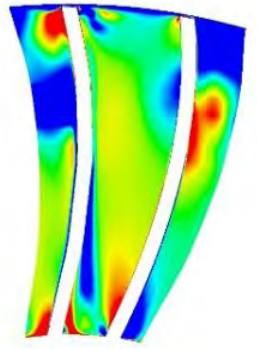

$35 \%$

Figure 7.28. Vorticity for various normalized chord lengths for tip gap (a) $1 \%$ and (b) $5 \%$. 
For inlet plane, the vorticity because of tiny gap is observed at case of tip clearance $1 \%$, while close to sharp blade corner there is a small region with opposite vorticity. For case of tip clearance $5 \%$ of blade height the vorticity seems to be insignificant but there are variations that can be observed for the flow in the gap. Then, as the flow is turning, the vorticity at the planes for chord $15 \%$ and $35 \%$ seems to be similar far from blade tip, but with some variations close to tip.

Three regions of the blade are studied, as they present the maximum condensation risk: (i) the leading edge, (ii) the suction side and (iii) the blade tip.

Finally, the static pressure distribution at the impeller is examined. Figure 7.29 presents the how the pressure varies for tip clearance 1\% (Figure 7.29a) and for $5 \%$ (Figure 7.29b). The regions where the supersaturation is presented to be more significant (thus condensation is starting) are investigated, so the post-processing tool was adjusted in order to illustrate as blue every region with supercritical pressure far from saturation curve (7.39 MPa).

Regarding the leading edge, it is noticed that for both tip clearance gaps, this region presents the minimum pressure. The tip gap $1 \%$ of blade height seems to present a minimum pressure around 5.5 MPa close to tip, while the tip gap 5\% presents a higher minimum pressure around $6 \mathrm{MPa}$. Although the fluid pressure is lower (green-yellow region) than the saturation pressure, the volume of the twophase flow seems to be small. Moreover, it is observed that for smaller tip clearance the pressure at suction side presents a profile with minimum at mid-span, while at hub and tip the pressure is getting higher. For the case of tip clearance $5 \%$ of blade height, the pressure seems to present another profile, having lower pressure region up to tip, but at the mid-span the expansion is not so extensive.

For decreasing blade tip clearance, the phenomena of the low pressure are less obvious at sharp corner of the main blade leading edge tip, which may reduce the effect of condensation. The reason is that when the blade tip clearance size is small, the distance between the casing and the blade tip is decreased. Close to main blade leading edge tip, the fluid flowing in the upward direction to the casing could be restrained, the shedding vortex at the blade leading edge tip could not be developed fully and the intensity and influence become powerless.

Despite the fact that the intensity is getting weak, the influenced area becomes larger, affecting the suction side of the blade. Consecutively, as the tip clearance gap increases, the low pressure and density phenomena do not present significant variation at the main blade leading edge tip, however the condensation is getting more concentrated and more severe at the sharp corner. Figure 7.29 presents this change of condensation effect at leading edge and at tip in detail between small and larger tip clearance. 


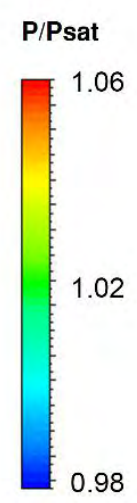

(a)

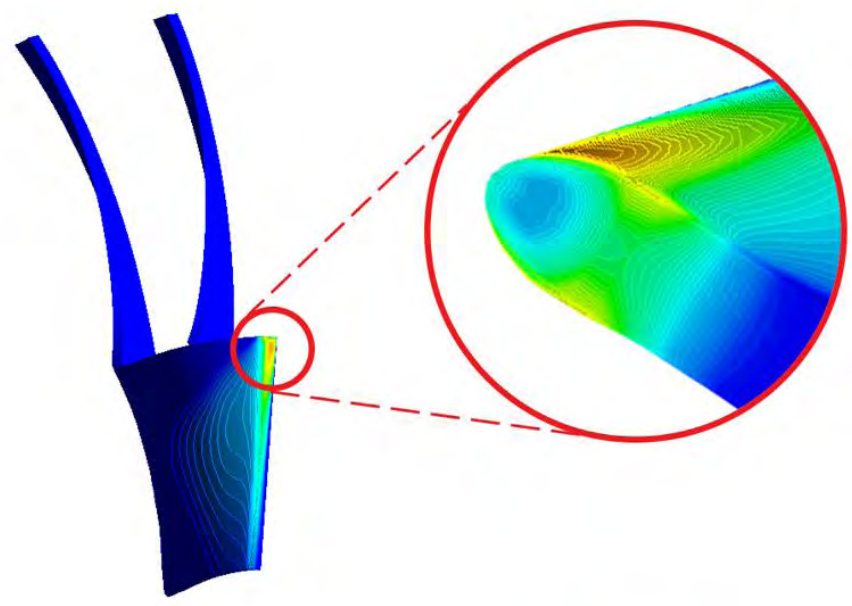

(b)

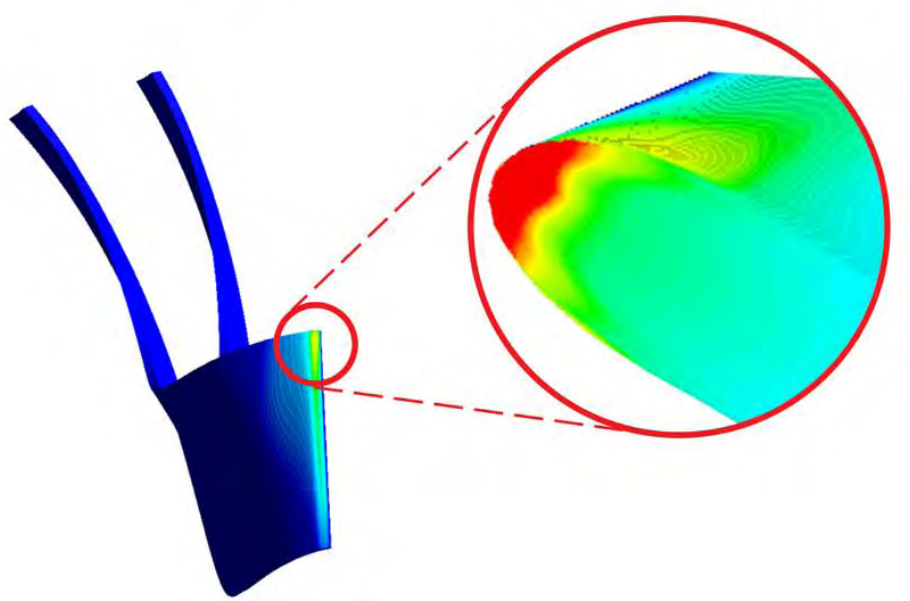

Figure 7.29.View of one blade from inlet for condensation effect at blade for tip clearance (a) $1 \%$ and (b) $5 \%$ of the blade height.

\subsection{Conclusions}

In this chapter the results of the centrifugal compressor's analysis are presented. Firstly, the results of the direct problem are validated with published data for centrifugal compressor referred as Model A, presenting good agreement with a $1.2 \%$ relative error for the outlet total pressure. Then, the results of the inverse problem are presented leading in a geometry design for the impeller and the diffuser in order to meet the thermodynamic properties derived by the thermodynamic analysis. An aerodynamic analysis employing computational fluid 
dynamics for the derived 3D geometry follows, investigating the influence of the real gas properties accuracy on the compressor performance.

Various external look-up tables used for the flow simulation were compared, leading in a real-gas properties (RGP) look-up table with 500 points around the critical point. It was observed that a look-up table with higher resolution around the critical table can lead in a more accurate prediction of the pressure distribution at the blade leading edge and the possible condensation.

The aerodynamic analysis leaded in a performance map for an operational range of the compressor with a maximum efficiency around $88 \%$. However, the ratio of the fluid pressure to the saturation pressure at the impeller's leading edge showed that the condensation effects can appear for high mass flow rates and rotational speeds, reducing the operational range of the compressor in a region that ensures the supercritical flow.

Finally, the tip clearance effect on the condensation was examined. The tip clearance $1 \%$ of the blade height used for design is extremely small to be achieved by compressor manufacturer. Therefore, a tip clearance that equals $1 \mathrm{~mm}$ (5\% of blade height) was investigated and the condensation presence at the blade leading edge, suction side and tip was studied. It was observed that the increase of the tip clearance gap, increases intensely the condensation at the corner of the tip, while for small tip clearance presents weaker effects but the influenced area becomes larger. 


\section{Chapter 8}

\section{Conclusions and Future Work}

Supercritical carbon dioxide cycles constitute a promising power generation technology, because of their high efficiency and compact size. This study examined the feasible operation limits of a recompression s- $\mathrm{CO}_{2}$ Brayton cycle, focusing on two critical components, namely, the high temperature recuperator and the compressor, in terms of design and off-design performance.

First, a thermodynamic analysis of a $600 \mathrm{MW}$ th recompression s- $\mathrm{CO}_{2}$ Brayton cycle was performed, in order to examine how main parameters of the cycle (such as compressor or turbine entry temperature) affect the cycle efficiency and the limits of the operational range. The maximum cycle efficiency reached $46.1 \%$ for a pressure ratio 2.55 and a turbine inlet temperature of $830 \mathrm{~K}$. The exergy analysis indicated that the high temperature recuperator produces the highest exergy losses for the cycle. Additionally, the thermal-hydraulic performance of a printed-circuit heat exchanger employed as a high-temperature recuperator was investigated. A one-dimensional segmental analysis was used in order to model the heat transfer and the pressure drop. Moreover, the results of experiments on the steady-state and transient operation of a PCHE of similar technology were employed for model validation. Finally, CFD analysis of a heat exchanger module was employed to investigate the full-scale performance of the high-temperature recuperator, with effectiveness increased to $98 \%$, which improves cycle efficiency for $0.1 \%$ for the design point.

The study also considered the centrifugal compressor design and performance, as the operation in the pseudo-critical region is highly affected by real-gas effects and the two-phase risk is high. A design method was presented, leading in a geometry for the above considered cycle. The real gas effects were studied for the centrifugal compressor, as the inlet conditions approach the critical point. A singlephase simulation (supercritical) is used for the CFD analysis far from critical point, as in the case of heat exchangers. However, for the compressor inlet look-up tables with extrapolation of the $\mathrm{s}-\mathrm{CO}_{2}$ properties onto the liquid domain and varying resolution were generated, giving the thermodynamic conditions close to critical point were used to check the simulation differences, resulting in a look-up table with 500 points around the critical point $(0.3 \%$ relative error for specific heat capacity) and 100 points in the region where carbon dioxide approaches perfect gas 
behavior. The aerodynamic performance of the centrifugal compressor stage was studied numerically, using the derived look-up table for the thermodynamic properties and the performance map is derived, while the possibility of two-phase flow is also taken into account. Condensation risk exists at the regions that the flow accelerates, thus the blade leading edge, the impeller suction side and the blade tip. The numerical study focused on the regions with high condensation risk, resulting in the operating conditions that lead in two-phase flow and finally a performance map with limited operational range was presented. Moreover, this study investigated how the tip clearance affects the condensation at the blade tip, showing that the increased tip clearance results in increased condensation at the blade tip sharp corner, while the smaller tip clearance reduces the condensation at the leading edge but increases the affected area.

The condensation at the impeller leading edge consists a significant challenge of the future compression systems, in order to prolong the compressor performing time and ensure a safe operation. The use of inlet guide vanes could resolve the problem of incidence at the compressor inlet. Besides, the inlet guide vanes would have a throttling impact, giving the opportunity to adjust the compressor range. However, these guide vanes introduce pressure drop to the system, which could lead to significant inconsistency both the compressor inlet pressure and the pressure of the whole cycle. Additionally, the use of multiple compression stages could lead to smaller flow acceleration at the compression inlet, but this choice increases significantly the system complexity, size that is the main cycle's advantage and cost investment.

Power cycles, such as the considered recompression $\mathrm{s}-\mathrm{CO}_{2}$ Brayton cycle, should have the possibility operating at full and part-load depending on the energy consumptions and the available thermal load. The current thesis stated some interesting points regarding the part-load operation, investigating the off-design performance of the printed-circuit heat exchangers and the performance of the system's centrifugal compressor. It is observed that although the cycle efficiency exceeds $46 \%$ for the design point, the heat exchangers' effectiveness and pressure drop are highly affected by the operating point variation, which leads to reduced cycle efficiency. Moreover, the results regarding the centrifugal compressor operating with supercritical carbon dioxide showed that at high mass flow rates, the condensation risk is getting more and more significant, restricting the compressor's operation at high mass flows. 


\subsection{Future Work}

The wide adoption of s- $\mathrm{CO}_{2}$ recompression Brayton cycles for power generation in the future requires significant effort regarding both printed-circuit heat exchangers and compressor stage. The following important issues must be further investigated in this respect for heat exchangers:

- Experiments for PCHE used data only at the inlet and outlet of the heat exchangers. The evolution of internal temperature field during transients could be studied by advanced techniques as infrared thermography, to further understand the pinch-point problem.

- Alternative design versions of PCHEs could be studied to assess their impact on cycle efficiency.

In the case of compression stage, the following issues should be examined further:

- The stall and choke margins of the compression stage should be investigated.

- Studying condensation during start-up for transient operation would give a significant standpoint regarding the compressor operating margins.

- The nucleation time scale in the metastable region for $\mathrm{CO}_{2}$ should be examined in order to assess the condensation risk.

- Stress analysis of the centrifugal compressor and study of its bearings' lubrication strategy.

- The possibility of employing an axial multistage compressor could be investigated. 


\section{Bibliography}

1. Feher E. G., The supercritical thermodynamic power cycle. Energy conversion, 1968. 8(2): p. 85-90.

2. Angelino G., Carbon dioxide condensation cycles for power production. Journal of Engineering for Power, 1968. 90(3): p. 287-295.

3. Dostal V., Driscoll M. J., Hejzlar P., A supercritical carbon dioxide cycle for next generation nuclear reactors. Massachusetts Institute of Technology. Dept. of Nuclear Engineering, Cambridge, MA, Paper No. MIT-ANP-TR-100, 2004.

4. Fleming D., Holschuh T., Conboy T., Rochau G., Fuller R. Scaling Considerations for a Multi-Megawatt Class Supercritical CO2 Brayton Cycle and Path Forward for Commercialization. in ASME Turbo Expo 2012.

5. Musgrove G., Rimpel A., Wilkes J. Tutorial: Fundamentals of Supercritical CO2 in Proceedings of Supercritical CO2 Power Cycle Symposium. 2016. San Antonio, TX.

6. Chacartegui R., De Escalona J. M., Sánchez D., Monje B., Sánchez T., Alternative cycles based on carbon dioxide for central receiver solar power plants. Applied Thermal Engineering, 2011. 31(5): p. 872-879.

7. Sánchez D., Brenes B. M., Muñoz de Escalona J. M., Chacartegui R., Nonconventional combined cycle for intermediate temperature systems. International Journal of Energy Research, 2013. 37(5): p. 403-411.

8. Besarati S. M.,Yogi Goswami D., Analysis of Advanced Supercritical Carbon Dioxide Power Cycles With a Bottoming Cycle for Concentrating Solar Power Applications. Journal of Solar Energy Engineering, 2013. 136(1): p. 010904-010904.

9. Iverson B. D., Conboy T. M., Pasch J. J., Kruizenga A. M., Supercritical CO2 Brayton cycles for solar-thermal energy. Applied Energy, 2013. 111: p. 957-970.

10. Milani D., Luu M. T., McNaughton R., Abbas A., A comparative study of solar heliostat assisted supercritical $\mathrm{CO} 2$ recompression Brayton cycles: Dynamic modelling and control strategies. The Journal of Supercritical Fluids, 2017. 120: p. 113-124.

11. Singh R., Miller S. A., Rowlands A. S., Jacobs P. A., Dynamic characteristics of a direct-heated supercritical carbon-dioxide Brayton cycle in a solar thermal power plant. Energy, 2013. 50: p. 194-204.

12. Turchi C. S., Ma Z., Neises T. W., Wagner M. J., Thermodynamic study of advanced supercritical carbon dioxide power cycles for concentrating solar power systems. Journal of Solar Energy Engineering, 2013. 135(4): p. 041007.

13. Jacob F., Rolt A., Sebastiampillai J., Sethi V., Belmonte M., Cobas P., Performance of a Supercritical CO2 Bottoming Cycle for Aero Applications. Applied Sciences, 2017. 7(3): p. 255.

14. Sienicki J. J., Moisseytsev A., Fuller R. L., Wright S. A., Pickard P. S. Scale Dependencies of Supercritical Carbon Dioxide Brayton Cycle Technologies and the Optimal Size for a Next-Step Supercritical CO2 Cycle Demonstration. in Supercritical CO2 Power Cycle Sympoisum. 2011. Boulder, USA.

15. Clementoni E. M.,Cox T. L. Steady-state power operation of a supercritical carbon dioxide Brayton cycle. in ASME Turbo Expo 2014: Turbine Technical Conference and Exposition. 2014. American Society of Mechanical Engineers. 
16. Clementoni E. M., Cox T. L., King M. A., Off-Nominal Component Performance in a Supercritical Carbon Dioxide Brayton Cycle. Journal of Engineering for Gas Turbines and Power, 2016. 138(1): p. 011703.

17. Kato Y., Nitawaki T., Muto Y., Medium temperature carbon dioxide gas turbine reactor. Nuclear Engineering and Design, 2004. 230(1): p. 195-207.

18. Ishiyama S., Muto Y., Kato Y., Nishio S., Hayashi T., Nomoto Y., Study of steam, helium and supercritical $\mathrm{CO} 2$ turbine power generations in prototype fusion power reactor. Progress in Nuclear Energy, 2008. 50(2): p. 325-332.

19. Ishizuka T., Muto Y., Aritomi M., Tsuzuki N., Kikura H., Design and Analysis of the Axial Bypass Compressor Blade of the Supercritical CO2 Gas Turbine. 2009(43512): p. 813-822.

20. Takagi K., Muto Y., Ishizuka T., Kikura H., Aritomi M., Research on Flow Characteristics of Supercritical CO2 Axial Compressor Blades by CFD Analysis. Journal of Power and Energy Systems, 2010. 4(1): p. 138-149.

21. Ahn Y., Bae S. J., Kim M., Cho S. K., Baik S., Lee J. I., Cha J. E., Review of supercritical $\mathrm{CO} 2$ power cycle technology and current status of research and development. Nuclear Engineering and Technology, 2015. 47(6): p. 647-661.

22. Ahn Y., Lee J., Kim S. G., Lee J. I., Cha J. E., Lee S.-W., Design consideration of supercritical CO2 power cycle integral experiment loop. Energy, 2015. 86: p. 115127.

23. Lee J., Lee J. I., Ahn Y., Yoon H. Design Methodology of Supercritical CO2 Brayton Cycle Turbomachineries. in ASME Turbo Expo 2012: Turbine Technical Conference and Exposition. 2012. American Society of Mechanical Engineers.

24. Bae S. J., Ahn Y., Lee J., Kim S. G., Baik S., Lee J. I., Experimental and numerical investigation of supercritical $\mathrm{CO} 2$ test loop transient behavior near the critical point operation. Applied Thermal Engineering, 2016. 99: p. 572-582.

25. Fuller R., Preuss J., Noall J. Turbomachinery for supercritical CO2 power cycles. in ASME Turbo Expo 2012: Turbine Technical Conference and Exposition. 2012. American Society of Mechanical Engineers.

26. Conboy T., Wright S., Pasch J., Fleming D., Rochau G., Fuller R., Performance characteristics of an operating supercritical $\mathrm{CO} 2$ Brayton cycle. Journal of Engineering for Gas Turbines and Power, 2012. 134(11): p. 111703.

27. Cha J. E., Bae S. W., Lee J., Cho S. K., Lee J. I., Park J. H. Operation Results of a Closed Supercritical CO2 Simple Brayton Cycle. in 5th International SymposiumSupercritical CO2 Power Cycles. 2016. San Antonio, TX, USA.

28. Wright S. A., Radel R. F., Vernon M. E., Rochau G. E., Pickard P. S., Operation and analysis of a supercritical CO2 brayton cycle. Sandia Report, No. SAND2010-0171, 2010.

29. Utamura M., Fukuda T., Aritomi M., Aerodynamic characteristics of a centrifugal compressor working in supercritical carbon dioxide. Energy Procedia, 2012. 14: p. 1149-1155.

30. Allam R., Martin S., Forrest B., Fetvedt J., Lu X., Freed D., Brown G. W., Sasaki T., Itoh M., Manning J., Demonstration of the Allam Cycle: An Update on the Development Status of a High Efficiency Supercritical Carbon Dioxide Power Process Employing Full Carbon Capture. Energy Procedia, 2017. 114: p. 5948-5966.

31. Held T. J. Initial Test Results of a Megawatt-class Supercritical CO2 heat engine. in 4th International Symposium on Supercritical CO2 Power Cycles, Pittsburgh, PA, Sept. 2014. 
32. Le Pierres R., Southall D., Osborne S. Impact of Mechanical Design Issues on Printed Circuit Heat Exchangers. in Supercritical CO2 Power Cycle Symposium, Boulder, CO, May. 2011.

33. Ding M., Liu J., Cheng W.-L., Huang W.-X., Liu Q.-N., Yang L., Liu S.-Y., An adaptive flow path regenerator used in supercritical carbon dioxide Brayton cycle. Applied Thermal Engineering, 2018. 138: p. 513-522.

34. Kim D. E., Kim M. H., Cha J. E., Kim S. O., Numerical investigation on thermalhydraulic performance of new printed circuit heat exchanger model. Nuclear Engineering and Design, 2008. 238(12): p. 3269-3276.

35. Xu X., Ma T., Li L., Zeng M., Chen Y., Huang Y., Wang Q., Optimization of fin arrangement and channel configuration in an airfoil fin PCHE for supercritical CO2 cycle. Applied Thermal Engineering, 2014. 70(1): p. 867-875.

36. Chen F., Zhang L., Huai X., Li J., Zhang H., Liu Z., Comprehensive performance comparison of airfoil fin PCHEs with NACA 00XX series airfoil. Nuclear Engineering and Design, 2017. 315: p. 42-50.

37. Cui X., Guo J., Huai X., Cheng K., Zhang H., Xiang M., Numerical study on novel airfoil fins for printed circuit heat exchanger using supercritical CO2. International Journal of Heat and Mass Transfer, 2018. 121: p. 354-366.

38. Southall D. Diffusion bonding in compact heat exchangers. in Proceedings of 2009 international congress on advances in nuclear power plants. 2009. Japan.

39. Southall D.,Dewson S. J., Innovative compact heat exchangers, in ICAPP10, San Diego, CA, USA, Paper 10300. 2010.

40. Heatric, https://www.heatric.com/index.html.

41. Wang C., Design, analysis and optimization of the power conversion system for the Modular Pebble Bed Reactor System, in Dept. of Nuclear Engineering. Massachusetts Institute of Technology, 2003.

42. Dyreby J., Klein S., Nellis G., Reindl D., Design Considerations for Supercritical Carbon Dioxide Brayton Cycles With Recompression. Journal of Engineering for Gas Turbines and Power, 2014. 136(10): p. 101701-101701-9.

43. Nikitin K., Kato Y., Ngo L., Printed circuit heat exchanger thermal-hydraulic performance in supercritical $\mathrm{CO} 2$ experimental loop. International Journal of Refrigeration, 2006. 29(5): p. 807-814.

44. Tsuzuki N., Kato Y., Ishiduka T., High performance printed circuit heat exchanger. Applied Thermal Engineering, 2007. 27(10): p. 1702-1707.

45. Ngo T. L., Kato Y., Nikitin K., Ishizuka T., Heat transfer and pressure drop correlations of microchannel heat exchangers with S-shaped and zigzag fins for carbon dioxide cycles. Experimental Thermal and Fluid Science, 2007. 32(2): p. 560570.

46. Kim I. H., No H. C., Lee J. I., Jeon B. G., Thermal hydraulic performance analysis of the printed circuit heat exchanger using a helium test facility and CFD simulations. Nuclear Engineering and Design, 2009. 239(11): p. 2399-2408.

47. Kim I. H.,No H. C., Thermal hydraulic performance analysis of a printed circuit heat exchanger using a helium-water test loop and numerical simulations. Applied Thermal Engineering, 2011. 31(17): p. 4064-4073.

48. Mylavarapu S., Sun X., Figley J., Needler N., Christensen R., Investigation of HighTemperature Printed Circuit Heat Exchangers for Very High Temperature Reactors. Journal of Engineering for Gas Turbines and Power, 2009. 131(6): p. 062905-0629057. 
49. Mylavarapu S. K., Sun X., Glosup R. E., Christensen R. N., Patterson M. W., Thermal hydraulic performance testing of printed circuit heat exchangers in a high-temperature helium test facility. Applied Thermal Engineering, 2014. 65(1): p. 605-614.

50. Kruizenga A., Li H., Anderson M., Corradini M., Supercritical Carbon Dioxide Heat Transfer in Horizontal Semicircular Channels. Journal of Heat Transfer, 2012. 134(8): p. 081802-081802-10.

51. Figley J., Sun X., Mylavarapu S. K., Hajek B., Numerical study on thermal hydraulic performance of a Printed Circuit Heat Exchanger. Progress in Nuclear Energy, 2013. 68: p. 89-96.

52. Yoon S.-J., Sabharwall P., Kim E.-S., Analytical Study on Thermal and Mechanical Design of Printed Circuit Heat Exchanger. 2013, Technical report, Idaho Nation Laboratory, Idaho Falls, 83415.

53. Baik S., Kim S. G., Bae S. J., Ahn Y., Lee J., Lee J. I. Preliminary experimental study of precooler in supercritical CO2 Brayton cycle. in ASME Turbo Expo 2015: Turbine Technical Conference and Exposition. 2015. American Society of Mechanical Engineers.

54. Schmitt J., Amos D., Kapat J. Design and Real Fluid Modelling of Micro-Channel Recuperators for a Nominal 100 MW Class Recuperated Recompression Brayton Cycle Using Supercritical Carbon Dioxide. in ASME Turbo Expo 2015: Turbine Technical Conference and Exposition. 2015. American Society of Mechanical Engineers.

55. Khan H. H., M A. A., Sharma A., Srivastava A., Chaudhuri P., Thermal-hydraulic characteristics and performance of 3D wavy channel based printed circuit heat exchanger. Applied Thermal Engineering, 2015. 87: p. 519-528.

56. Aneesh A. M., Sharma A., Srivastava A., Vyas K. N., Chaudhuri P., Thermalhydraulic characteristics and performance of 3D straight channel based printed circuit heat exchanger. Applied Thermal Engineering, 2016. 98: p. 474-482.

57. Chen M., Sun X., Christensen R. N., Skavdahl I., Utgikar V., Sabharwall P., Pressure drop and heat transfer characteristics of a high-temperature printed circuit heat exchanger. Applied Thermal Engineering, 2016. 108: p. 1409-1417.

58. Meshram A., Jaiswal A. K., Khivsara S. D., Ortega J. D., Ho C., Bapat R., Dutta P., Modeling and analysis of a printed circuit heat exchanger for supercritical $\mathrm{CO} 2$ power cycle applications. Applied Thermal Engineering, 2016.

59. Guo J., Design analysis of supercritical carbon dioxide recuperator. Applied Energy, 2016. 164: p. 21-27.

60. Kim S. G., Lee Y., Ahn Y., Lee J. I., CFD aided approach to design printed circuit heat exchangers for supercritical CO2 Brayton cycle application. Annals of Nuclear Energy, 2016. 92: p. 175-185.

61. Yoon S.-J., O'Brien J., Chen M., Sabharwall P., Sun X., Development and validation of Nusselt number and friction factor correlations for laminar flow in semi-circular zigzag channel of printed circuit heat exchanger. Applied Thermal Engineering, 2017. 123: p. 1327-1344.

62. Baik S., Kim S. G., Lee J., Lee J. I., Study on $\mathrm{CO} 2$ - water printed circuit heat exchanger performance operating under various $\mathrm{CO}_{2}$ phases for $\mathrm{S}-\mathrm{CO}_{2}$ power cycle application. Applied Thermal Engineering, 2017. 113: p. 1536-1546.

63. Chu W.-X., Li X.-h., Ma T., Chen Y.-t., Wang Q.-w., Experimental investigation on $\mathrm{SCO} 2$-water heat transfer characteristics in a printed circuit heat exchanger with straight channels. International Journal of Heat and Mass Transfer, 2017. 113: p. 184194. 
64. Aneesh A. M., Sharma A., Srivastava A., Chaudhury P., Effects of wavy channel configurations on thermal-hydraulic characteristics of Printed Circuit Heat Exchanger (PCHE). International Journal of Heat and Mass Transfer, 2018. 118: p. 304-315.

65. Sharifi F., Golkar Narandji M. R., Mehravaran K., Dynamic simulation of plate heat exchangers. International Communications in Heat and Mass Transfer, 1995. 22(2): p. 213-225.

66. Carstens N., Control Strategies for Supercritical Carbon Dioxide Power Conversion Systems, Ph.D. Dissertation Massachusetts Institute of Technology, 2007.

67. Pra F., Tochon P., Mauget C., Fokkens J., Willemsen S., Promising designs of compact heat exchangers for modular HTRs using the Brayton cycle. Nuclear Engineering and Design, 2008. 238(11): p. 3160-3173.

68. Chen M., Sun X., Christensen R. N., Shi S., Skavdahl I., Utgikar V., Sabharwall P., Experimental and numerical study of a printed circuit heat exchanger. Annals of Nuclear Energy, 2016. 97: p. 221-231.

69. Chen M., Sun X., Christensen R. N., Skavdahl I., Utgikar V., Sabharwall P., Dynamic behavior of a high-temperature printed circuit heat exchanger: Numerical modeling and experimental investigation. Applied Thermal Engineering, 2018. 135: p. 246-256.

70. Jiang Y., Liese E., Zitney S. E., Bhattacharyya D., Design and dynamic modeling of printed circuit heat exchangers for supercritical carbon dioxide Brayton power cycles. Applied Energy, 2018. 231: p. 1019-1032.

71. Park J. H., Bae S. W., Park H. S., Cha J. E., Kim M. H., Transient analysis and validation with experimental data of supercritical $\mathrm{CO} 2$ integral experiment loop by using MARS. Energy, 2018. 147: p. 1030-1043.

72. Balje O. E., Turbomachines - A guide to design, selection, and theory. 1981: John Wiley \&amp; Sons.

73. Gong Y., Carstens N., Driscoll M., Matthews I., Analysis of radial compressor options for supercritical $\mathrm{CO} 2$ power conversion cycles. Massachusetts, USA: Center for Advanced Nuclear Energy Systems, MIT Department of Nuclear Science and Engineering, 2006.

74. Whitfield A.,Baines N. C., Design of radial turbomachines. 1990: New York, NY (USA); John Wiley and Sons Inc.; None. Medium: X; Size: Pages: (480 p).

75. Denton J. D., Loss Mechanisms in Turbomachines. Journal of Turbomachinery, 1993.

76. Oh H. W., Yoon E. S., Chung M. K., An optimum set of loss models for performance prediction of centrifugal compressors. Proceedings of the Institution of Mechanical Engineers, Part A: Journal of Power and Energy, 1997. 211(4): p. 331-338.

77. Japikse D., Centrifugal compressor design and performance. 1996.

78. Aungier R. H., Centrifugal compressors: a strategy for aerodynamic design and analysis. 2000: American Society of Mechanical Engineers.

79. Monge Brenes B., Design of supercritical carbon dioxide centrifugal compressors, in University of Seville. 2014.

80. Moore J. J.,Nored M. G., Novel Concepts for the Compression of Large Volumes of Carbon Dioxide. 2008(43178): p. 645-653.

81. Moore J. J., Lerche A., Allison T., Moreland B., Pacheco J., Development of an Internally Cooled Centrifugal Compressor for Carbon Capture and Storage Applications. 2012(44717): p. 997-1006.

82. Monje B., Sánchez D., Savill M., Pilidis P., Sánchez T. A design strategy for supercritical CO2 compressors. in ASME Turbo Expo 2014: Turbine Technical Conference and Exposition. 2014. American Society of Mechanical Engineers.

83. Schlichting H., Boundary Layer Theory. 6th Edition ed, ed. McGraw-Hill. 1968, New York. 
84. Rinaldi E., Pecnik R., Colonna P. Steady state CFD investigation of a radial compressor operating with supercritical CO2. in ASME Turbo Expo 2013: Turbine Technical Conference and Exposition. 2013. American Society of Mechanical Engineers.

85. Zhao H., Deng Q., Zheng K., Zhang H., Feng Z. Numerical Investigation on the Flow Characteristics of a Supercritical CO2 Centrifugal Compressor. in ASME Turbo Expo 2014: Turbine Technical Conference and Exposition. 2014. American Society of Mechanical Engineers.

86. Zhao H., Deng Q., Zhang H., Feng Z., The Influence of Tip Clearance on Supercritical CO2 Centrifugal Compressor Performance. 2015(56802): p. V009T36A008.

87. Pham H. S., Alpy N., Ferrasse J. H., Boutin O., Tothill M., Quenaut J., Gastaldi O., Cadiou T., Saez M., An approach for establishing the performance maps of the sc$\mathrm{CO} 2$ compressor: Development and qualification by means of CFD simulations. International Journal of Heat and Fluid Flow, 2016.

88. Liu Z., Luo W., Zhao Q., Zhao W., Xu J., Preliminary Design and Model Assessment of a Supercritical CO2 Compressor. Applied Sciences, 2018. 8(4): p. 595.

89. Pecnik R., Rinaldi E., Colonna P., Computational fluid dynamics of a radial compressor operating with supercritical CO2. Journal of Engineering for Gas Turbines and Power, 2012. 134(12): p. 122301.

90. Baltadjiev N. D., Lettieri C., Spakovszky Z. S., An Investigation of Real Gas Effects in Supercritical CO2 Centrifugal Compressors. Journal of Turbomachinery, 2015. 137(9): p. 091003-091003-13.

91. Ameli A., Turunen-Saaresti T., Backman J., Numerical Investigation of the Flow Behavior Inside a Supercritical CO2 Centrifugal Compressor. 2016(49873): p. V009T36A014.

92. Lüdtke K. H., Process Centrifugal Compressors, Springer, 2003. Chapter 2.

93. Span R.,Wagner W., A new equation of state for carbon dioxide covering the fluid region from the triple-point temperature to $1100 \mathrm{~K}$ at pressures up to $800 \mathrm{MPa}$. Journal of physical and chemical reference data, 1996. 25(6): p. 1509-1596.

94. Klein S.,Alvarado F., F-chart Software. EES, Madison, WI, 2014.

95. Oh C. H., Kim E. S., Patterson M., Design Option of Heat Exchanger for the Next Generation Nuclear Plant. Journal of Engineering for Gas Turbines and Power, 2009. 132(3): p. 032903-032903.

96. Gkountas A. A., Stamatelos A. M., Kalfas A. I., Recuperators investigation for high temperature supercritical carbon dioxide power generation cycles. Applied Thermal Engineering, 2017. 125: p. 1094-1102.

97. Gnielinski V., New equations for heat and mass-transfer in turbulent pipe and channel flow. International chemical engineering, 1976. 16(2): p. 359-368.

98. Hesselgreaves J. E., Law R., Reay D., Compact heat exchangers: selection, design and operation. 2016: Butterworth-Heinemann.

99. Anselmi E., Brunce I., Pachidis V., Zachos P., Johnston M. An Overview of the RollsRoyce sCO2-Test Rig Project at Cranfield University. in 6th International Supercritical CO2 Power Cycles Symposium, March. 2018. Pittsburgh, Pennsylvania.

100. American Society of Mechanical Engineers A., Performance Test Code for Single Phase Heat Exchangers, ASME PTC 12.5-2000.

101. JCGM100, Evaluation of measurement data- Guide to the Expression of Uncertainty in Measurements. 2008.

102. Stefopoulos G., Rigas S., Tsirikoglou P., Kalfas A.I. Evaluation of Pressure and Species Concentration Measurement using Uncertainty Propagation. in Measuring Techniques in Turbomachinery. 2014. Lyon, France. 
103. Lemmon E. W., Huber M. L., McLinden M. O., NIST Reference Fluid Thermodynamic and Transport Properties_-REFPROP. 2013.

104. Behr T., Kalfas A. I., Abhari R. S., A probabilistic uncertainty evaluation method for turbomachinery probe measurement, in The XVIII Symposium on Measuring Techniques in Turbomachinery Transonic and Supersonic Flow in Cascades and Turbomachines, September 21-22, 2006.

105. Conrad O., Raif K., Wessels M. The calculation of performance maps for centrifugal compressors with vane-island diffusers, in Proceedings of the Twenty-fifth Annual International Gas Turbine Conference and Exhibit and Twenty-second Annual Fluids Engineering Conference, New Orleans, La., March 9-13, 1980.

106. Aungier R., Mean Streamline Aerodynamic Performance Analysis of Centrifugal Compressors. Journal of Turbomachinery, 1995. 117: p. 361.

107. Dixon S. L.,Hall C. A., Chapter 7 - Centrifugal Pumps, Fans, and Compressors, in Fluid Mechanics and Thermodynamics of Turbomachinery (Sixth Edition). 2010, Butterworth-Heinemann: Boston. p. 217-263.

108. Krain H., Review of Centrifugal Compressor's Application and Development. Journal of Turbomachinery, 2005. 127(1): p. 25-34.

109. Wiesner F. J., A Review of Slip Factors for Centrifugal Impellers. Journal of Engineering for Power, 1967. 89(4): p. 558-566.

110. Boyce M. P., Centrifugal compressors: a basic guide, ed. Books P. 2003.

111. Daily J. W.,Nece R. E., Chamber Dimension Effects on Induced Flow and Frictional Resistance of Enclosed Rotating Disks. Journal of Basic Engineering, 1960. 82(1): p. 217-230.

112. Aungier R. H., Aerodynamic Design and Analysis of Vaneless Diffusers and Return Channels. 1993(78880): p. V001T03A042.

113. Johnston J. P.,Dean J. R. C., Losses in Vaneless Diffusers of Centrifugal Compressors and Pumps: Analysis, Experiment, and Design. Journal of Engineering for Power, 1966. 88(1): p. 49-60.

114. Ryzhov Y. A., Pirumov U. G., Gorbunov V. N., Nonequilibrium condensation in highspeed gas flows. 1984.

115. VDI Heat Atlas. VDI Heat Atlas. 2010: Springer Berlin Heidelberg.

116. Wright S. A., Pickard P. S., Fuller R., Radel R. F., Vernon M. E. Supercritical CO2 Brayton Cycle Power Generation Development Program and Initial Test Results. in ASME Power Conference, 2009. 


\section{Nomenclature}

\begin{tabular}{|c|c|c|}
\hline Symbol & Parameter & Units \\
\hline \multirow[t]{2}{*}{$\mathrm{a}$} & i) Speed of sound & {$[\mathrm{m} / \mathrm{s}]$} \\
\hline & ii) Heat transfer coefficient & {$\left[\mathrm{W} / \mathrm{m}^{2} \cdot \mathrm{K}\right]$} \\
\hline$\dot{C}$ & Heat capacitance rate & \\
\hline$C_{r}$ & Heat capacitance ratio & \\
\hline$C_{t h}$ & Thermal mass of heat exchanger & {$[\mathrm{J} / \mathrm{kg} \cdot \mathrm{K}]$} \\
\hline$D_{s}$ & Specific diameter & \\
\hline$D_{\text {hyd }}$ & Hydraulic diameter & {$[\mathrm{m}]$} \\
\hline$\dot{E}$ & Exergy rate & {$[\mathrm{W}]$} \\
\hline$\dot{I}$ & Irreversibility & {$[\mathrm{W}]$} \\
\hline$I_{c}$ & Work input coefficient & \\
\hline$L_{C}$ & Impeller pressure loss coefficient & \\
\hline$L_{P}$ & Length of blade pitch & {$[\mathrm{m}]$} \\
\hline$M W_{t h}$ & Thermal MW (power unit of thermal load) & \\
\hline$M W_{e}$ & Electrical MW (power unit of power generation) & \\
\hline$N_{S}$ & Specific speed & \\
\hline$R_{\text {tot }}$ & Heat transfer resistance & {$[\mathrm{K} / \mathrm{W}]$} \\
\hline$S_{i j}$ & Deformation rate tensor & {$\left[\mathrm{s}^{-1}\right]$} \\
\hline$T_{o}$ & Total temperature & {$[\mathrm{K}]$} \\
\hline$Z_{b}$ & Number of blades & \\
\hline$c_{p}$ & Specific heat capacity under constant pressure & {$[\mathrm{J} / \mathrm{kg} \cdot \mathrm{K}]$} \\
\hline$c_{r}$ & Radial velocity in stationary reference frame & {$[\mathrm{m} / \mathrm{s}]$} \\
\hline$c_{\theta}$ & Tangential velocity in stationary reference frame & {$[\mathrm{m} / \mathrm{s}]$} \\
\hline$d_{h}$ & Hydraulic diameter & {$[\mathrm{m}]$} \\
\hline$\dot{m}$ & Mass flow rate & {$[\mathrm{kg} / \mathrm{s}]$} \\
\hline$n_{s}$ & Isentropic exponent & \\
\hline$\dot{q}$ & Heat load rate & {$\left[\mathrm{W} / \mathrm{m}^{2}\right]$} \\
\hline $\mathrm{s}-\mathrm{CO}_{2}$ & Supercritical carbon dioxide & \\
\hline$u^{+}$ & Dimensionless velocity & \\
\hline$y^{+}$ & Wall y-plus & \\
\hline$\beta_{T}$ & Isothermal compressibility & \\
\hline
\end{tabular}


$\beta_{p} \quad$ Isobaric compressibility

$\delta_{i j} \quad$ Kronecker delta

$\tau_{w} \quad$ Wall shear stress

$\left[\mathrm{N} / \mathrm{m}^{2}\right]$

Ideal part of Helmholtz function

Residual part of Helmholtz function

Specific enthalpy

$[\mathrm{J} / \mathrm{kg}]$

A

Cross section area

$\left[m^{2}\right]$

$B$

Blockage factor

$D$

Impeller diameter

[m]

$I$

Rothalpy

K

Total pressure loss coefficient apart from impeller

$L$

Length

[m]

M

Mach number

N

Rotational speed

[rpm]

$\mathrm{Nu}$

Nusselt number

$P$

Pressure

$[\mathrm{Pa}]$

$\mathrm{Pr} \quad$ Prandtl number

$R$

Degree of reaction

$R e$

Reynolds number

$\mathrm{S}$

Supersaturation

Static temperature

[K]

$U$

Circumferential velocity

[m/s]

$U A$

Overall heat transfer coefficient

[W/K]

Z

Compressibility factor

a

Flow angle

[deg]

$b$

Blade height

[m]

c Absolute velocity

$[\mathrm{m} / \mathrm{s}]$

$f$

Friction factor

$k$

Thermal conductivity

$[\mathrm{W} / \mathrm{m} \cdot \mathrm{K}]$

$S$

Specific entropy

$[\mathrm{J} / \mathrm{kg} \cdot \mathrm{K}]$

$t$

Blade thickness

[m]

$v$

Fluid velocity

[m/s]

w

Relative velocity in stationary reference frame

$[\mathrm{m} / \mathrm{s}]$ 


\section{Greek symbols}

$\begin{array}{lll}\Phi & \text { Flow coefficient } & \\ \Psi & \text { Stage coefficient } & \text { [deg] } \\ \alpha & \text { Flow angle } & {[\mathrm{deg}]} \\ \beta & \text { Blade angle } & \\ \gamma & \text { Ratio of specific heat } & \\ \varepsilon & \text { Effectiveness } & \\ \eta & \text { Efficiency } & {[\mathrm{Pa} \cdot \mathrm{s}]} \\ \lambda & \text { Distortion factor } & {\left[\mathrm{kg} / \mathrm{m}^{3}\right]} \\ \mu & \text { Dynamic viscosity } & \\ \rho & \text { Density } & \\ \sigma & \text { Slip factor } & \\ \varphi & \text { Dimensionless Helmholtz function } & \\ \omega & \text { i) Pressure loss coefficient } & {\left[\mathrm{s}^{-1}\right]}\end{array}$

\section{Subscript}

$\begin{array}{ll}\text { B } & \text { Blade } \\ \text { ch } & \text { Cold } \\ \text { cl } & \text { Channel } \\ \text { comp } & \text { Clearance } \\ \text { cont } & \text { Compressor } \\ \text { DF } & \text { Contraction } \\ \text { h } & \text { Disk friction } \\ \text { i } & \text { Hot } \\ \text { id } & \text { Numbering of components or subheaters } \\ \text { imp } & \text { Ideal } \\ \text { in } & \text { Impeller } \\ \text { inc } & \text { Inlet } \\ \text { fr } & \text { Incidence } \\ \text { mc } & \text { Friction } \\ \text { min } & \text { Main compressor } \\ \text { out } & \text { Minimum } \\ \mathrm{P} & \text { Outlet } \\ \text { prec } & \text { Solid plate } \\ \text { RC } & \text { Pre-cooler } \\ \text { recomp } & \text { Recirculation } \\ & \text { Recompressor }\end{array}$




$\begin{array}{ll}\text { rel } & \text { Relative } \\ \text { th } & \text { Thermal } \\ \text { turb } & \text { Turbine } \\ \text { ts } & \text { Total to static } \\ \text { tt } & \text { Total to total } \\ \text { wf } & \text { Working fluid }\end{array}$

\section{$\underline{\text { Abbreviations }}$}

EES Engineering Equation Solver

EOS Equation Of State

HTR High Temperature Recuperator

LTR Low Temperature Recuperator

MPCHE Modified Printed-Circuit Heat Exchanger

NTU Number of Transfer Units

PCHE Printed-Circuit Heat Exchangers

RANS Reynolds Averaged Navier Stokes 


\section{Appendix - A1}

\section{Uncertainty Analysis}

The calculation of some parameters measured in the experiment such as the uncertainty of temperature, pressure and mass flow and their impact on enthalpy change and finally on heat transfer are given below. Table A1-1 presents the variable error of the measurements for case 1 presented in Chapter 6.

Table A1-1. Estimation of errors associated with the measurement of heat transfer for case 1.

\begin{tabular}{ccccc}
\hline Parameter & $\begin{array}{c}\text { Measurement } \\
\text { device }\end{array}$ & $\begin{array}{c}\text { Approximate } \\
\text { value }\end{array}$ & $\begin{array}{c}\text { Variable } \\
\text { error }\end{array}$ & $\begin{array}{c}\delta \mathrm{x} / \mathrm{x} \\
(\%)\end{array}$ \\
\hline$T_{\text {in }}$ & Thermocouple & $346.7 \mathrm{~K}$ & $0.2 \mathrm{~K}$ & 0.058 \\
$T_{\text {out }}$ & Thermocouple & $325.6 \mathrm{~K}$ & $0.2 \mathrm{~K}$ & 0.061 \\
$P_{\text {in }}$ & Piezo resistive & $8.37 \mathrm{MPa}$ & $20.9 \mathrm{kPa}$ & $0.25 \%$ \\
$P_{\text {out }}$ & Piezo resistive & $8.31 \mathrm{MPa}$ & $20.7 \mathrm{kPa}$ & $0.25 \%$ \\
$\dot{m}$ & Coriolis flow meter & $0.1 \mathrm{~kg} / \mathrm{s}$ & $0.0035 \mathrm{~kg} / \mathrm{s}$ & $0.35 \%$ \\
$\Delta p_{\text {air }}$ & $\begin{array}{c}\text { Differential pressure } \\
\text { gauge }\end{array}$ & $1070 \mathrm{~Pa}$ & $3.2 \mathrm{~Pa}$ & $0.3 \%$ \\
\hline
\end{tabular}

The uncertainty for measuring the pressure drop and the heat transfer was presented in Chapter 4.5.1 and the differential parameters for density and enthalpy have to be estimated.

$$
\begin{aligned}
& \delta \rho=\left\{\left[\left(\frac{\partial \rho}{\partial p}\right) \delta p\right]^{2}+\left[\left(\frac{\partial \rho}{\partial T}\right) \delta T\right]^{2}\right\}^{1 / 2} \\
& \delta h=\left\{\left[\left(\frac{\partial h}{\partial T}\right) \delta T\right]^{2}+\left[\left(\frac{\partial h}{\partial p}\right) \delta p\right]^{2}\right\}^{1 / 2}
\end{aligned}
$$

where the derivative $\partial h / \partial T$ equals specific heat capacity under constant pressure $c_{p}$, while all the other derivatives for density and pressure can be estimated using the Refprop database. 
Therefore, the errors associated with the measurements for the four cases used in experimental tests (Chapter 6.3.1) regarding enthalpy and heat transfer are presented below.

Table A1-2. Estimation of errors associated with the measurement of heat transfer for case 1.

\begin{tabular}{lcccc}
\hline \multicolumn{1}{c}{ Parameter } & Case 1 & Case2 & Case 3 & Case4 \\
\hline$\delta h_{\text {in }}(\mathrm{J} / \mathrm{kg})$ & 334.3 & 345.6 & 305.4 & 378.9 \\
$\delta h_{\text {out }}(\mathrm{J} / \mathrm{kg})$ & 506 & 540.5 & 382.6 & 503.8 \\
$\delta Q / Q$ & $1.52 \%$ & $1.68 \%$ & $1.44 \%$ & $2.22 \%$ \\
\hline
\end{tabular}

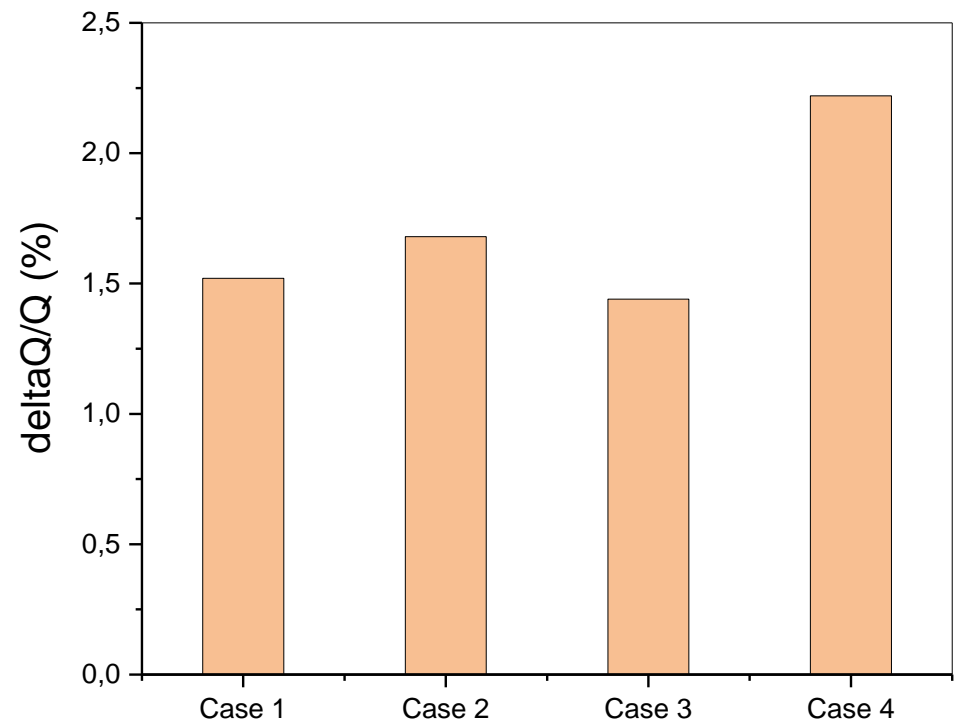

Figure A1-1. Relative error of measured heat transferred. 


\section{List of Publications}

1. Polyzos N. D., Gkountas A. A., Kalfas A. I., Design and Performance of a Fan for Aeronautical Applications, ASME Turbo Expo, Lillestrøm, Norway, 2018

2. Gkountas, A. A., Stamatelos A. M., Kalfas A. I., Recuperators investigation for high temperature supercritical carbon dioxide power generation cycles. Applied Thermal Engineering, 2017. 125: p. 1094-1102.

3. Gkountas, A. A., Stamatelos A. M., Kalfas A., Thermodynamic Modeling and Comparative Analysis of Supercritical Carbon Dioxide Brayton Cycle, ASME Turbo Expo, Charlotte (NC), USA, 2017

4. Dermata T.K., Schina L., Gkountas A. A., Andritsos N. A., Investigation of a Natural Gas / Low Enthalpy Geothermal Energy Hybrid System, European Geothermal Congress, Strasbourg, France, 2016 
